$$
{\stackrel{*}{*} \operatorname{sit}^{* *}}_{*}^{*}
$$
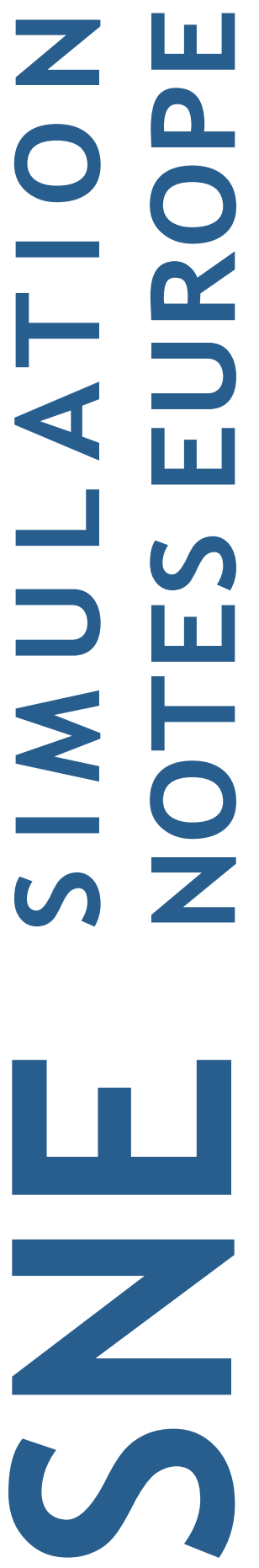

Special Issue EUROSIM Promotion
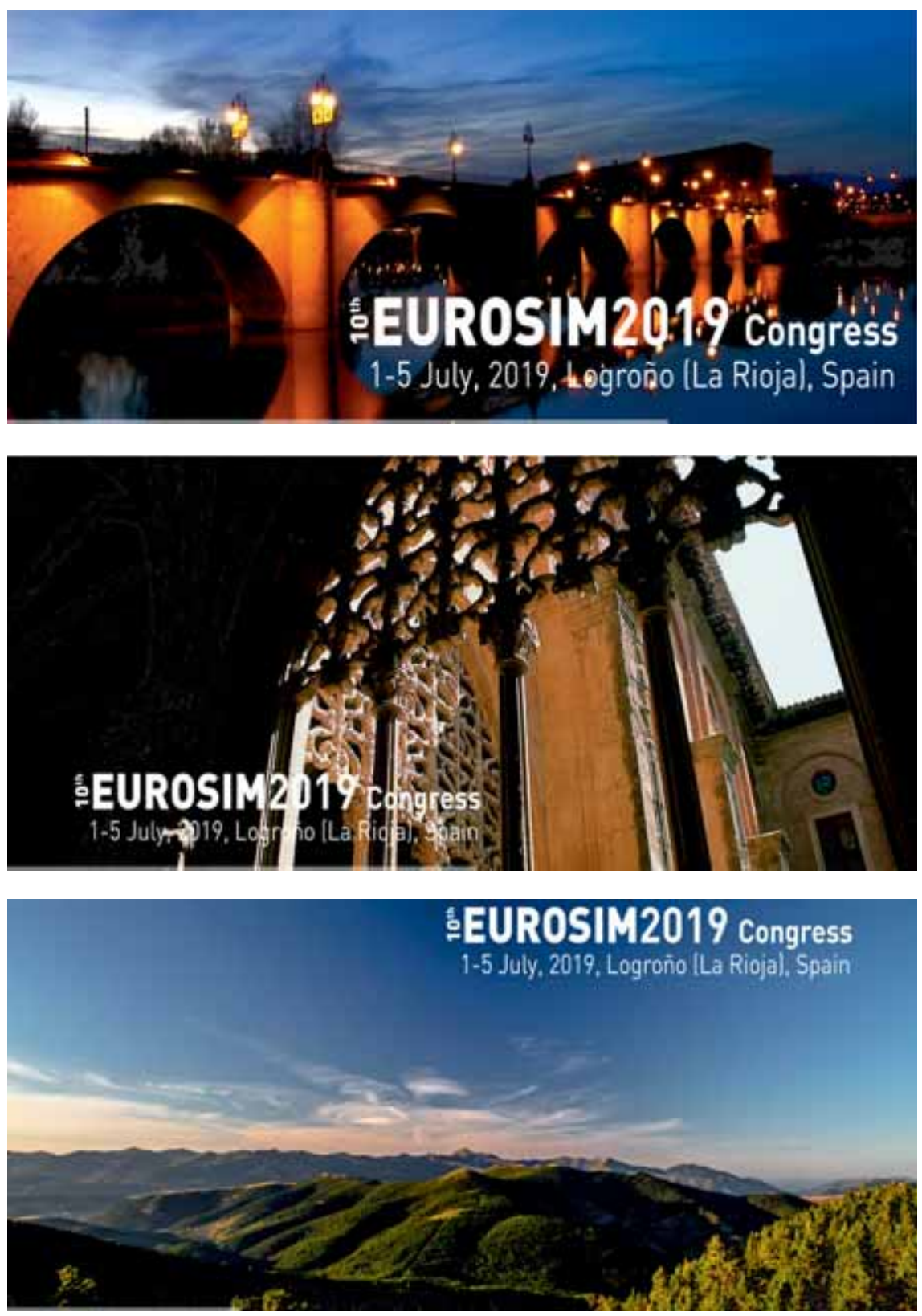

Journal on Developments and Trends in Modelling and Simulation EUROSIM Scientific Membership Journal

Vol. 28 No.4, Dec. 2018 ISSN Online 2306-0271 ISSN Print 2305-9974

DOI 10.11128/sne.28.4.1044 ISBN Print 978-3-903024-77-9 


\section{SNE Aims \& Scope}

Simulation Notes Europe (SNE) provides an international, high-quality forum for presentation of new ideas and approaches in simulation - from modelling to experiment analysis, from implementation to verification, from validation to identification, from numerics to visualisation - in context of the simulation process.

SNE seeks to serve scientists, researchers, developers and users of the simulation process across a variety of theoretical and applied fields in pursuit of novel ideas in simulation and to enable the exchange of experience and knowledge through descriptions of specific applications. SNE follows the recent developments and trends of modelling and simulation in new and/or joining application areas, as complex systems and big data. SNE puts special emphasis on the overall view in simulation, and on comparative investigations, as benchmarks and comparisons in methodology and application. For this purpose, SNE documents the ARGESIM Benchmarks on Modelling Approaches and Simulation Implementations with publication of definitions, solutions and discussions. SNE welcomes also contributions in education in/for/with simulation.

A News Section in SNE provides information on EUROSIM Simulation Societies and Simulation Groups.

SNE, primarily an electronic journal, follows an open access strategy, with free download in basic layout. SNE is the official membership journal of EUROSIM, the Federation of European Simulation Societies and Simulation Groups www.eurosim.info. Members of EUROSIM societies are entitled to download SNE in an elaborate and extended layout, and to access additional sources of benchmark publications, model sources, etc. Print SNE is available for specific groups of EUROSIM societies, and starting with Volume 27 (2017) as printon-demand from TU Verlag, TU Wien. SNE is DOI indexed by CrossRef, identified by DOI prefix 10.11128 , assigned to the SNE publisher ARGESIM (www.argesim.org).

Author's Info. Individual submissions of scientific papers are welcome, as well as post-conference publications of contributions from conferences of EUROSIM societies. SNE welcomes special issues, either dedicated to special areas and/or new developments, or on occasion of events as conferences and workshops with special emphasis.

Authors are invited to submit contributions which have not been published and have not being considered for publication elsewhere to the SNE Editorial Office.

SNE distinguishes different types of contributions (Notes), i.e.

- TN Technical Note, 6-10 p. - EN Education Note, 6-10 p.

- PN Project Note 6-10 p. - SN Short Note, max. 6 p.

- SW Software Note , 4-6 p. - BN Benchmark Note, 2- 10 p.

- ON Overview Note - only upon invitation, up to $14 \mathrm{p}$.

Further info and templates (doc, tex) at SNE's website. www.sne-journal.org

\section{SNE Editorial Board}

SNE - Simulation Notes Europe is advised and supervised by an international scientific editorial board. This board is taking care on peer reviewing of submissions to SNE.

Felix Breitenecker, Felix.Breitenecker@tuwien.ac.at TU Wien, Math. Modelling, Austria, Editor-in-chief

DavidAl-Dabass, david.al-dabass@ntu.ac.uk, Nottingham Trent University, UK

Maja Atanasijevic-Kunc,maja.atanasijevic@fe.uni-lj.si Univ. of Ljubljana, Lab. Modelling \& Control, Slovenia

Aleš Belič, ales.belic@sandoz.com Sandoz / National Inst. f. Chemistry, Slovenia

Peter Breedveld, P.C.Breedveld@el.utwente.nl University of Twenty, Netherlands

Agostino Bruzzone, agostino@itim.unige.it Universita degli Studi di Genova, Italy

Francois Cellier,fcellier@inf.ethz.ch, ETH Zurich, Switzerland

Vlatko Čerić,vceric@efzg.hr, Univ. Zagreb, Croatia

Russell Cheng,rchc@maths.soton.ac.uk University of Southampton, UK

Roberto Cianci,cianci@dime.unige.it, Math. Eng. and Simulation, Univ. Genova, Italy

Eric Dahlquist, erik.dahlquist@mdh.se, Mälardalen Univ., Sweden Umut Durak, umut.durak@dlr.de German Aerospace Center (DLR)Braunschweig, Germany

Horst Ecker,Horst.Ecker@tuwien.ac.at TU Wien, Inst. f. Mechanics, Austria

Vadim Engelson, vadim.engelson@mathcore.com MathCore Engineering, Linköping, Sweden

Peter Groumpos, groumpos@ece.upatras.gr Univ. of Patras, Greece

Edmond Hajrizi, ehajrizi@ubt-uni.net University for Business and Technology, Pristina, Kosovo

Glenn Jenkins, GLJenkins@ cardiffmet.ac.uk Cardiff Metropolitan Univ., UK

Emilio Jiminez,emilio.jimenez@unirioja.es University of La Rioja, Spain

Esko Juuso, esko.juuso@oulu.fi Univ. Oulu, Dept. Process/Environmental Eng., Finland

Kaj Juslin, kaj.juslin@enbuscon.com, Enbuscon Ltd, Finland Andreas Körner, andreas.koerner@tuwien.ac.at TU Wien, Math. E-Learning Dept., Vienna, Austria

Francesco Longo,f.longo@unical.it Univ. of Calabria, Mechanical Department, Italy

Yuri Merkuryev, merkur@itl.rtu.lv, Riga Technical Univ.

David Murray-Smith,d.murray-smith@elec.gla.ac.uk University of Glasgow, Fac. Electrical Engineering, UK

Gasper Music,gasper.music@fe.uni-lj.si Univ. of Ljubljana, Fac. Electrical Engineering, Slovenia

Thorsten Pawletta, thorsten.pawletta@hs-wismar.de Univ. Wismar, Dept. Comp. Engineering, Wismar, Germany

Niki Popper,niki.popper@dwh.at, dwh Simulation Services, Austria

Kozeta Sevrani, kozeta.sevrani@unitir.edu.al Univ. Tirana, Inst.f. Statistics, Albania

Thomas Schriber, schriber@umich.edu University of Michigan, Business School, USA

Yuri Senichenkov, sneyb@dcn.infos.ru St. Petersburg Technical University, Russia

Michal Štepanovský, stepami9@fit.cvut.cz. Technical Univ. Prague, Czech Republic

Oliver Ullrich, oliver.ullrich@iais.fraunhofer.de Fraunhofer IAIS, Germany

Siegfried Wassertheurer, Siegfried.Wassertheurer@ait.ac.at AIT Austrian Inst. of Technology, Vienna, Austria

Sigrid Wenzel, S.Wenzel@uni-kassel.de Univ. Kassel, Inst. f. Production Technique, Germany Grégory Zacharewicz, gregory.zacharewicz@mines-ales.fr IMT École des Mines d'Alès, France 


\section{Editorial}

Dear Readers - This SNE Special Issue 'EUROSIM Promotion' was initiated by the EUROSIM Board on the occasion of the board meeting in Budapest, September 2018. Its aim is to promote the various activities of EUROSIM: the EUROSIM Congress, publications of activities of EUROSIM member societies in SNE, and the publication of the ARGESIM/EUROSIM Benchmarks. Representatives of the active EUROSIM societies have compiled a very interesting SNE issue. The electronic version of this issue will be distributed via the EUROSIM mailing list and the printed version will be part of the handouts at the EUROSIM Congress 2019 in Spain (see next page). Two novelties are coming along with this issue. First, the contributions to the ARGESIM Benchmarks are enriched by a broader variety of contribution types (Solution - Report - Study) and by an educational component (as it has turned out that the Benchmark Definitions are frequently used as basis for exercises in simulation education (details see the benchmark overview contribution in this issue). And second, authors now can submit SNE contributions directly via the SNE website (www.sne-journal.org/contribute-contact/).

I would like to thank all authors for their contributions, and I would like to thank the Special Issue Editors for their very successful work (due to limited space some nominated contributions had to be postponed for publication in the next SNE issue). And last but not least thanks to the SNE Editorial Office for layout, typesetting, and publishing work with DOI indexing - allowing publication in time.

Felix Breitenecker, SNE Editor-in-Chief, eic@sne-journal.org; felix.breitenecker@tuwien.ac.at

\section{Contents SNE 28(4) Special Issue 'EUROSIM Promotion'}

Online SNE 28(4) DOI 10.11128/ sne.28.4.1044

Print SNE 28(4) Print-on-Demand ISBN 978-3-903024-77-9

Print-on-Demand, TU Verlag Vienna, www.tuverlag.at

A Learned Polyalphabetic Decryption Cipher.

C. Hewage, A. Jayal, G. Jenkins, R. J. Brown

Generating of Task-Based Controls for Joint-Arm

Robots with Simulation-Based Reinforcement Learning.

G. Kunert, T. Pawletta

Modeling Arterial Wave Reflection with Difference Equations. L. Lotteraner, B. Hametner, S. Wassertheurer...... 157

Autonomous Landing System: Safe Landing Zone Identification. A. Blažič, K. Kotnik, K. Nikolovska, M. Ožbot, M. Pernuš, U. Petkovič, N. Hrušovar, M. Verbič, I. Ograjenšek, A. Zdešar, M. Bošnak, T. Tomažič, G. Klančar

Different Strategies for Modelling and Simulation of the Impact of Migration on Regional Population Development. M. Obermair, M. Bicher, F. Breitenecker

Decision Trees for Human Activity Recognition Modelling in Smart House Environments. V.G. Sánchez, N..O. Skeie 177

Benefit Analysis of Wind Energy Storage by Time Shift Simulation. D. Andor, A. Aquino, E. Martínez-Cámara, J. I. Latorre, E. Jiménez

A Simulation-based Analysis of the Influence of Renewables on the Spanish Electricity Prices. D. Azofra, E. Jiménez, E. Martínez-Cámara, J. Blanco, J. I. Latorre

Modeling and Validation of an Open-Source Mean Value Heavy-Duty Diesel Engine Model.

K. Ekberg, V. Leek, L. Eriksson

Time- and Event-oriented Spreadsheet Modelling of ARGESIM Benchmark C12 'Collision of Spheres'. Z. Kolozsvari, L. Lienbacher, S. Perebner, H. Teixeira Rego, M. Bicher, A. Körner, H. Ecker, F. Breitenecker
Educational Use of the ARGESIM Benchmarks for Modelling Approaches and Simulation Implementations.

F. Breitenecker, A. Körner, H. Ecker 215

EUROSIM Societies Short Info $\mathrm{N} 1$ News EUROSIM, ASIM, DBSS, SLOSIM ………......... N9 Info and Call EUROSIM Congress 2019 $\mathrm{N} 13$

\section{SNE Contact \& Info}

\section{SNE Online ISSN 2306-0271, SNE Print ISSN 2305-9974}

$\rightarrow$ www.sne-journal.org

埄= office@sne-journal.org, eic@sne-journal.org

\section{$\bowtie$ SNE Editorial Office}

Johannes Tanzler (Layout, Organisation), Irmgard Husinsky (Web, Electronic Publishing), Felix Breitenecker (Organisation, Authors) ARGESIM / M ath. M odelling \& Simulation Group, Inst. of Analysis and Scientific Computing, TU Wien Wiedner Hauptstrasse 8-10, 1040 Vienna , Austria

\section{SNE SiMULATION NOTES EUROPE}

WEB: $\rightarrow$ www.sne-journal.org, DOI prefix 10.11128/sne

Scope: Developments and trends in modelling and simulation in various areas and in application and theory; comparative studies and benchmarks (documentation of ARGESIM Benchmarks on modelling approaches and simulation implementations); modelling and simulation in and for education, simulation-based e-learning; society information and membership information for EUROSIM members (Federation of European Simulation Societies and Groups).

Editor-in-Chief: Felix Breitenecker, TU Wien, M ath. Modelling Group

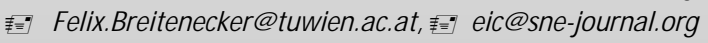

Print SNE and Print-on-Demand: Grafisches Zentrum and TU-Verlag, TU Wien, Wiedner Hauptstrasse 8-10, 1040, Vienna, Austria www.tuverlag.at

Publisher: ARGESIM ARBEITSGEM EINSCHAFT SIMULATION NEWS c/O M ath. Modelling and Simulation Group,

TU Wien / 101, Wiedner Hauptstrasse 8-10,

1040 Vienna, Austria; www.argesim.org, $=$ 夆 info@ argesim.org

on behalf of ASIM www.asim-gi.org and EUROSIM

$\rightarrow$ www.eurosim.info

(c) ARGESIM / EUROSIM / ASIM 2018 


\section{Editorial SNE Special Issue 'EUROSIM Promotion'}

\section{Dear Readers,}

This SNE issue SNE 28(4) is intended to promote the efforts of EUROSIM in distributing information on development on modelling and simulation - by means of the EUROSIM Congress, by means of EUROSIM's scientific journal SNE Simulation Notes Europe, by means of the work in EUROSIM's member societies, and by means of the EUROSIM/ARGESIM Benchmarks on Modelling Approaches and Simulation Implementations.

The triennial EUROSIM Congress is the main event of EUROSIM, the Federation of European Simulation Societies and Simulation Groups, with a long tradition since 1992, or 1986 resp., organized by one of EUROSIM's member societies. For July 2019 the Spanish Simulation Society CEASM SG invites experts in modelling and simulation to participate in the $10^{\text {th }}$ EUROSIM Congress in Logroño, La Rioja, Spain. The organizers are preparing a thrilling programme: science, culture and social events (visit the congress website www.eurosim 2019.com or visit the News Section in this issue). EUROSIM and EUROSIM's member societies are promoting the congress by several activities. This SNE special issue will be part of the congress handouts, intending to attract authors to publish in EUROSIM's scientific journal, which is also open for post-conference publication of contributions to conferences of EUROSIM Societies.

Members of the EUROSIM Societies are working in very different thematic areas of modelling and simulation, and in different working environments - industry, research centers, universities, development companies, etc. For this SNE special issue, the publication coordinators of the most active societies have selected contributions which show this broad spectrum of modelling and simulation.

CEA-SMSG, the Spanish Modelling and Simulation Group - organizer of EUROSIM Congress 2019, has selected contributions on modelling and simulation of energy production and energy consumption: benefit analysis of wind energy storage, and analysis of the influence of renewables on electricity prices.

SIMS, the Scandinavian Simulation Society (acting in Sweden, Norway, Denmark, Finland, and Iceland), is contributing with papers on activity recognition modelling in smart houses, and on modeling a heavy-duty Diesel engine (both being postconference publications of the SIMS 2018 Conference).

ASIM, the German Simulation Society (acting in Germany, Austria, and Switzerland), has selected contributions on mechatronics, population dynamics, and physiology: task-based controls for joint-arm robots with simulationbased reinforcement learning, impact of migration on regional population development, and arterial wave reflection modelling by difference equation.
UKSIM's contribution addresses the machine learning area, presenting a learned polyalphabetic decryption cipher - UKSIM is active in Great Britain and on international level.

The contributions of SLOSIM, the Slovenian Simulation Society, deal with safe landing zone identification for autonomous systems (this issue), and with multiscale simulation of metallurgical processes (next issue).

DBSS, the Dutch Benelux Simulation Society, candidate organizer of the EUROSIM 2022 Congress, has decided for a contribution on a simulation-based framework for establishing capacity boundaries at airports (next issue).

ARGESIM, the Austrian non-profit society for organization of simulation activities and publisher of SNE, is presenting three contributions on the ARGESIM Benchmarks. The first contribution, an educational benchmark study, presents spreadsheet modelling of ARGESIM Benchmark C12 Collision of Spheres. The second contribution is a short note on educational aspects of the benchmarks, and in the next issue an overview contribution on development, classification and possibilities for educational use of the benchmarks.

Due to limited space, already announced contributions from KA-SIM, the Kosovo Simulation Society, from LSS, the Latvian Simulation Society, and from CSSS, the Czech and Slovak Simulation Society, as well as further contributions to the ARGESIM Benchmarks will be published in the next issue - where also contributions from other active EUROSIM Societies are expected: LIOPHANT and MIMOS (Italy), NSSM (Russia), and PSCS (Poland).

The editors of this special issue would like to thank all colleagues from the EUROSIM Societies for selecting the contributions, and for providing the peer review. Furthermore, the editors express their hope that this issue indeed promotes the EUROSIM activities and animates simulationists to attend the EUROSIM Congress 2019 in Spain.

Emilio Jiménez, EUROSIM President, Organizer EUROSIM Congress 2019 in La Rioja, Spain (CEA-SMSG)

Juan Ignacio Latorre, Organizer EUROSIM Congress 2019 in La Rioja, Spain (CEA-SMSG)

David Al-Dabass, co-organizer EUROSIM Congress Cardiff (UKSIM)

Felix Breitenecker, EUROSIM Treasurer, Past President, Organizer EUROSIM Congress Vienna (ASIM)

Esko Juuso, EUROSIM Past President, Organizer EUROSIM Congress Oulu (SIMS

Andreas Körner, EUROSIM Board Secretary, Organizer MATHMOD Conference Series (ASIM, ARGESIM)

Miguel Mujica Mota, EUROSIM Secretary, Candidate Organizer EUROSIM Congress 2022 (DBSS)

Borut Zupančič, EUROSIM Past President, Organizer EUROSIM Congress Ljubljana (SLOSIM)

We hope to see you all at the EUROSIM Congress 2019! 


\title{
A Learned Polyalphabetic Decryption Cipher
}

\author{
Chaminda Hewage ${ }^{1 *}$, Ambikesh Jayal ${ }^{1}$, Glenn Jenkins ${ }^{1}$, Ryan J. Brown ${ }^{1}$ \\ ${ }^{1}$ Cardiff School of Technology, Cardiff Metropolitan University, Llandaff Campus, CF5 2YB, Cardiff, UK; \\ *chewage@cardiffmet.ac.uk
}

SNE 28(4), 2018, 141 - 148, DOI: 10.11128/sne.28.tn.10441

Received: November 20, 2018; Revised: December 6, 2018;

Accepted: December 8, 2018

SNE - Simulation Notes Europe, ARGESIM Publisher Vienna

ISSN Print 2305-9974, Online 2306-0271, www.sne-journal.org

\begin{abstract}
This paper examines the use of machine learning algorithms to model polyalphabetic ciphers for decryption. The focus of this research is to train and evaluate different machine learning algorithms to model the polyalphabetic cipher. The algorithms that have been selected are: (1) hill climbing; (2) genetic algorithm; (3) simulated annealing; and (4), random optimisation. The resulting models were deployed in a simulation to decrypt sample codes. The resulting analysis showed that the genetic algorithm was the most effective technique used in with hill climbing as second. Furthermore, both have the potential to be useful for larger problems.
\end{abstract}

\section{Introduction}

Stamp [1] states that: 'Cryptology is the art and science of making and breaking "secret codes". Martin [2] defines cryptology as a loosely used term to describe, 'the design and analysis of mechanisms based on mathematical techniques' to secure data and information.

There are two types of studies in cryptology. "Cryptography" describes the fundamentals of securing data by using such mechanisms to design an algorithm [2][15]. "Cryptanalysis" is the opposite of cryptography and uses an 'analysis of such mechanisms' to decrypt its encryption [2][14]. Cryptology is therefore a way of transforming an original message into cipher text that an interceptor may not be able to read and understand. However, the true recipient of the message could transform the message back to its original readable message by using a suitable decryption technique.

The purpose of cryptanalysis is to uncover or exploit encrypted information, it is a study and science of recovering the original plaintext without knowing the key [3]. Cryptanalysis is perceived today as 'code-breaking' or 'hacking', but maybe better known as an 'attack' [3][13]. Its primary concern is identifying and attacking the vulnerabilities in weak methods to gain knowledge of the plaintext [4].
As a cryptanalyst, it is important to understand what type of algorithm is used before attempting to unravel the cipher and give meaning to the content. According to Schneier [3] if a cryptanalyst cannot break the algorithm used, having known the background information of the algorithm, then they are unlikely to be successful at breaking it. Therefore, before any cryptanalyst can 'attack' an encrypted message, it is important to discover and analyse the type of method used for the cryptosystem.

The primary aim of this research is to explore the application of machine learning algorithms to the modelling polyalphabetic substitution ciphers for decryption. The focus of this paper is the application of well-known machine learning techniques as a first step to exploring more sophisticated machine learning techniques. The paper is structured as follows, first literature related to the cryptography techniques and machine learning algorithms are considered. Next the methodology used in is reported followed by the results. These are discussed and conclusions drawn in the final section.

\section{Machine Learning Algorithms}

Machine Learning is a branch of artificial intelligence, and its purpose is finding out if a computer can develop a model without prior learning and then improve this model, just like a human. The computer learns over time, which helps in finding a better solution to a problem [5], i.e. improving the model. More importantly, it learns 'without being explicitly programmed', which means that it has the ability to learn (create a model) based one dataset and apply this model to other datasets, the result is more flexible solutions [5]. Machine Learning research is a popular approach to problems today, such as: discovering new medicines and accurately diagnosing patients; working out better solutions to a specific problem (e.g. the travelling sales man); and finally, using machine learning to better understand cryptology.

Some machine learning algorithms are inspired by nature as these can provide a useful way of looking at a particular problem. 
This means that nature-inspired algorithms can be employed to achieve solutions to difficult tasks [6][12]. For example, the ant colony optimisation is a nature inspired algorithm analysing ants 'social behaviour in finding shortest paths' [6]; this type of algorithm could also help by solving other real world issues that require finding the shortest route.

This research takes into consideration three main machine learning nature-inspired algorithms. These are outlined below.

\subsection{Hill Climbing Algorithm}

This algorithm is inspired by nature as it 'resembles trying to find the top of Mount Everest in a thick fog while suffering from amnesia' [7]. The purpose of this type of algorithm is to find and improve on the best local solution to the problem after each step, checking whether the neighbouring results are better or worse than the current position (also known as a 'local search') [9]. A problem with this algorithm, is that it can reach a local maximum quite quickly. It may have found a good enough solution, but not the best (global maxima). Ways in which to resolve these issues include using multi-starts or simply allowing the algorithm to accept negative moves [8]. In turn, the hill climbing algorithm has more scope with the data, and has a better chance of finding a best solution.

\subsection{Genetic Algorithm}

Genetic Algorithm (GA) is another well-known nature inspired method; it is also known as an evolutionary approach. In particular, GA is inspired by biological evolution. For example, in the gene selection stage it takes both parents genes to produce a mutation or crossover to a new set of genetic composition [6]. It is a very useful algorithm for solving 'local search, optimisation and machine learning problems' [6]. This type of algorithm works by finding the best successor (result) from a combination of parents that are modified in ways of either mutation or crossover [7].

\subsection{Simulated Annealing}

The simulated annealing algorithm is based upon the process of heating up metals and glass to very high temperatures and slowly cooling them to the shape required [7]. This is quite similar to the hill climbing algorithm, but is implemented to prevent reaching a local maximum.

While the temperature is still high the probability allows the annealing process to accept worse answers, as well as a better ones.
This improves the scope in which the algorithm can search and as it leads towards a good solution the probability of accepting worse solutions are discarded.

\section{Methodology}

The aim of this research was to ascertain which of the selected machine learning algorithms were best at modelling this type of encryption. The following sections introduce the encryption algorithm used, test data selected, machine learning algorithms and metrics.

\subsection{Encryption}

A message from Winston Churchill to Franklin D. Roosevelt was used to create a sense of realism to the projects goal of uncovering sensitive data. For this to work, all grammar and spaces were removed from the text when encrypting, making the output text (cipher text) a single block of unreadable script. This ensured that the text is more difficult to comprehend and provided slightly more protection.

Encryption used a polyalphabetic cipher (Vigenere cipher) to encrypt the message, implemented based on well known sources [2,9]. This encryption focused on using a stream cipher to look at each individual bit of character of the text, and changed it to a new corresponding letter. An alternative method considered used an array of alphabetic letters that linked to an index; however, this method seemed to be less practical and consequently was not used.

\subsection{Decryption}

The procedure of decryption is very similar to encryption, as it performs a sequential search through each character of the cipher text, discovering the cipher text and keys represented decimal numbers of ASCII at each iteration. The outputted cipher text was used for decrypting to help analyse the machine learning algorithms, checking whether the cipher text can be decrypted. The purpose of the research was to find out whether the algorithms used can decrypt the cipher text with the same key as encrypted. Therefore, getting the algorithm to understand a way of finding the best decryption key. It is also worth pointing out that the key size has been hard coded into the program enabling the algorithms to work at an efficient rate, and focus on the decryption of the cipher text. 


\begin{tabular}{|c|c|c|c|}
\hline Algorithm & $\begin{array}{l}\text { Parameter } \\
\text { Name }\end{array}$ & Description & Value \\
\hline $\begin{array}{l}\text { Hill } \\
\text { Climbing }\end{array}$ & Repeats & $\begin{array}{l}\text { Number of times to re- } \\
\text { peat the experiment. }\end{array}$ & 10 \\
\hline \multirow[t]{5}{*}{$\begin{array}{l}\text { Genetic } \\
\text { Algorithm }\end{array}$} & Repeats & $\begin{array}{l}\text { Number of times to re- } \\
\text { peat the experiment. }\end{array}$ & 10 \\
\hline & $\begin{array}{l}\text { Population } \\
\text { Size }\end{array}$ & $\begin{array}{l}\text { Size of the population } \\
\text { of each generation. }\end{array}$ & 100 \\
\hline & $\begin{array}{l}\text { Mutation } \\
\text { Probability }\end{array}$ & Probability of mutation. & 0.6 \\
\hline & Elite & $\begin{array}{l}\text { Proportion of popula- } \\
\text { tion used for crossover. }\end{array}$ & 0.4 \\
\hline & Iterations & $\begin{array}{l}\text { Number of generations } \\
\text { over which to evolve } \\
\text { the population. }\end{array}$ & 120 \\
\hline \multirow[t]{2}{*}{$\begin{array}{l}\text { Simulated } \\
\text { Annealing }\end{array}$} & Repeats & $\begin{array}{l}\text { Number of times to re- } \\
\text { peat the experiment. }\end{array}$ & 10 \\
\hline & Initial Temp. & Initial temperature. & $\begin{array}{l}1.0 \mathrm{x} \\
10^{5}\end{array}$ \\
\hline \multirow[t]{2}{*}{$\begin{array}{l}\text { Random } \\
\text { Optimization }\end{array}$} & Repeats & $\begin{array}{l}\text { Number of times to re- } \\
\text { peat the experiment. }\end{array}$ & 10 \\
\hline & Iterations & $\begin{array}{l}\text { Maximum number of } \\
\text { training iterations. }\end{array}$ & 1000 \\
\hline
\end{tabular}

Table 1: The training parameters of the experiment.

\subsection{Scoring}

To test the algorithms, it was important to utilise a scoring method, which could assess each of the algorithms performances in modelling the key. The fitness of the decrypted text was scored using quad grams [9]. Quad grams were chosen over trigrams or bigrams in order to reduce the time taken for assessing score for the selected scope and length of the cipher text. This provided a metric to assess results of the English language decryption of the cipher text. Big O notation [11], provides a measure of complexity and gives an indication of how well an algorithm will scale. This was used to analyse the machine learning techniques and provide an indication of the algorithms potential.

\subsection{Machine Learning Algorithms}

The machine learning algorithms were implemented based on the work of Segaran [10], the training parameters used (shown in Table 1) were identified by experimentation.

\section{Results}

The results reported below are based on the the cipher score. Here each key modelled by the selected machine learning techniques is used to decrypt the cipher text and find its fitness score (closer to 0 represents the English language). The results showing the key used, completion time and the number of keys identified throughout the process (i.e. the number of times the best key changed). Each experiment was repeated ten times to ensure consistency of the results, these are recorded individually below so the keys can be visually compared.

\subsection{Random Hill Climbing}

The hill climbing algorithm has had the ability to get very close to the correct key. For example, this can be shown in repeat five of Figure 1, where the best score is a model with the an output (WINSTUN). This bares close resemblance to the key used (WINSTON) to encrypt, and the decrypted text will have close similarity to the text used. Considering all the outputs from this algorithm in Table 2 , they all reasonably close. This would suggest that using these keys for decryption would help a cryptanalysis to identify some words or letters in the cipher text. Consequently, this type of algorithm has fared considerably well in the short amount of time taken to nearly decrypt the message. This suggests that if this runs longer period of time, it could potentially model the key used and uncover the original plaintext.

The results in Table 2 suggests that this algorithm has the capability produce the best result due to the number of good models it can find in quite a short period. For example, repeats 8 with an output of 'ACNSTON' has the shortest completion time of 48.30 (s), but has found more than six thousand better models throughout the process. This implies that with only two letters wrong in this key that this decryption could be somewhat successful as some words or letters would be recognisable.

Also, due to the nature of this algorithm constantly striving to find a better result it has nearly achieved its full potential. Although it did not find the correct key within the repeats tested, it nevertheless came close to solving it.

The graphs in Figure 1 represent each of the repeats from Table 2. It is interesting to note that the graphs can be represented as sound waves, but are a collection of all the different hill climbing points within the scope.

All the lowest points in the graphs represent the starting point from where the algorithm grows to a better result, which is shown at the peaks throughout the processes.

Fascinatingly, it is not clear where the best result will be found and image 5 of Figure 1 clearly shows this, since within the process a sudden move to the best result noted. This is better than any other keys that have been found. Observing these graphs has helped to clarify the progress of the algorithms at each repeat. 


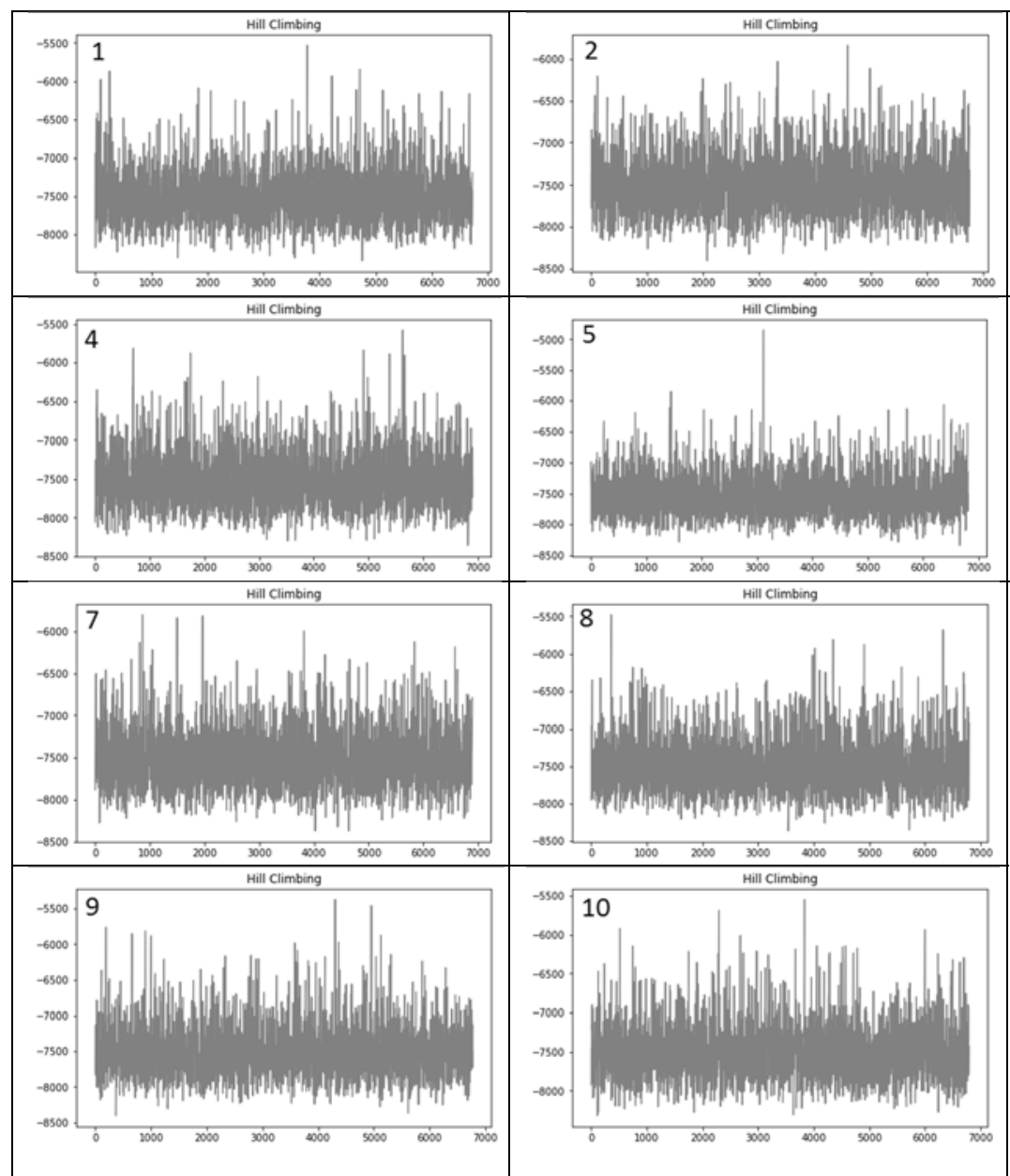

Figure 1: Random Hill Climbing Graphs

(Y-axis: Score; X-axis: No. of Best Solutions Found).

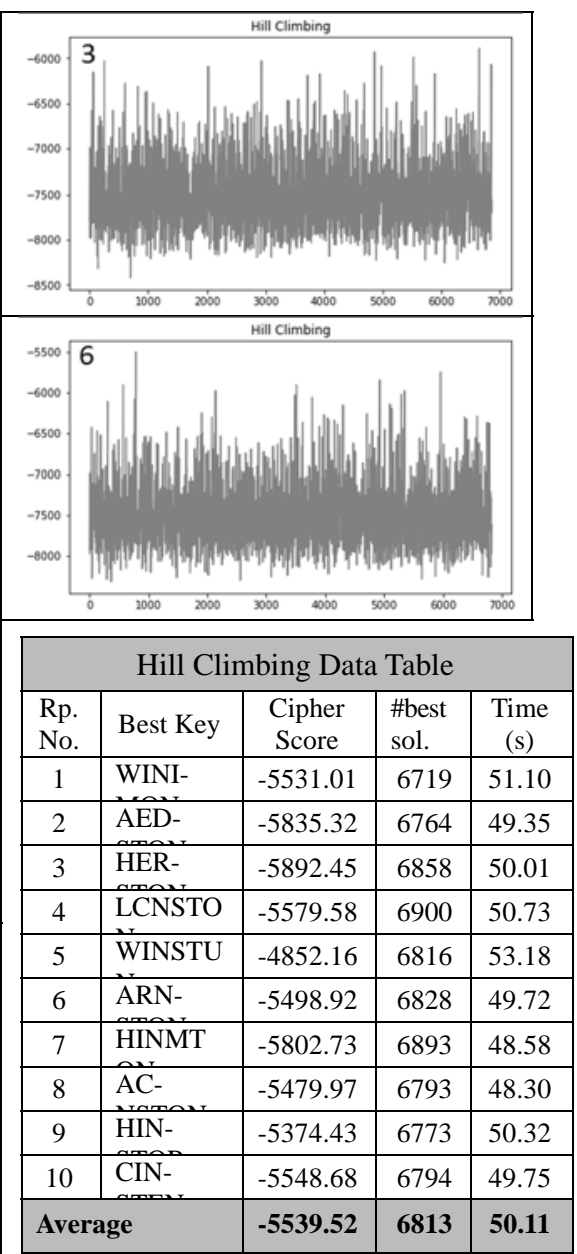

Table 2: Random Hill Climbing data.
Assessing the implementation of the algorithm the complexity is of quadratic-time $\mathrm{O}\left(\mathrm{N}^{2}\right)$. This suggests a increase in the size of the input (the key size) would results in a significant increase in complexity and therefore time to complete. This suggests that while the algorithm is suitable for the small keys used in this research there may be issues when scaling to larger keys.

\subsection{Genetic Algorithm}

The genetic algorithm results shown in Table 3, produces an accurate model of the key on three occasions. It can find the key used to encrypt the polyalphabetic cipher. Also, considering the other keys, it has a success rate correctly modelling at least five out of seven letters of the key. This largely suggests that using any of these keys could uncover some words or phrases in the decrypted text.
The results also show that for each repeat the number of best solutions has been reasonably consistent, this is ascribed to the underlying algorithm and its implementation. As shown in Table 3, this algorithm finds a good number of best solutions in a short period on average 23.40 (s).

The below graphs from Figure 2 represent the data collected in Table 3. There is a clear pattern to the graphs, the results demonstrate a consistent improvement in the result. For example, repeat 1 in Figure 2 has a rapid growth of finding the best result after 1000 other better solutions. However, a plateau appears once it has found its best solution.

This rectangular shape is a fluctuation between one best solution and another, and suggests that it cannot improve beyond this point (as shown in all other repeats). Looking back at the data in Figure 2 shows that once it is found what it thinks is the best solution of best keys, it does not have the capabilities to be able to progress beyond that. 


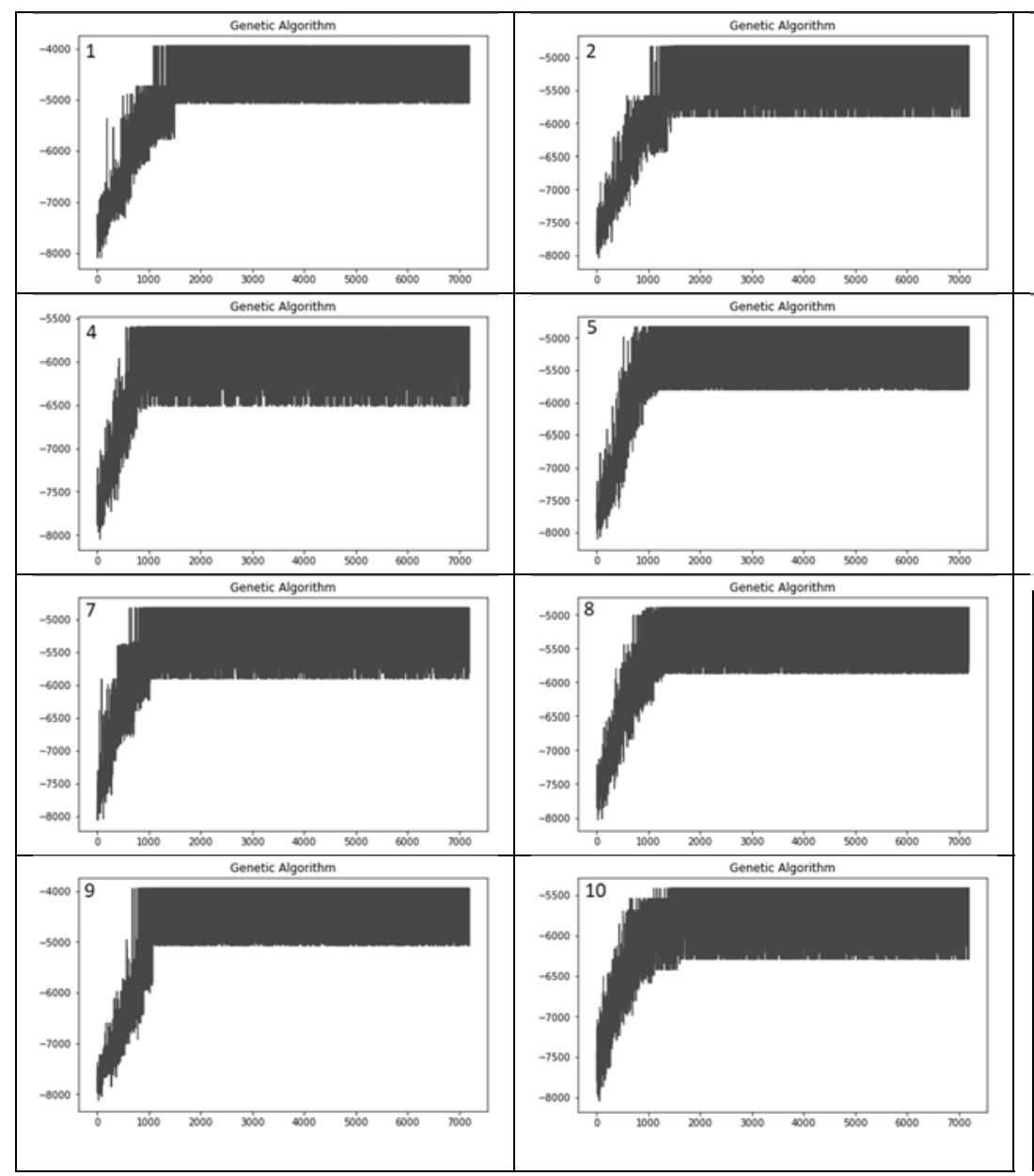

Figure 2: Genetic Algorithm Graphs (Y-axis: Score; X-axis: No. of Best Solutions Found).

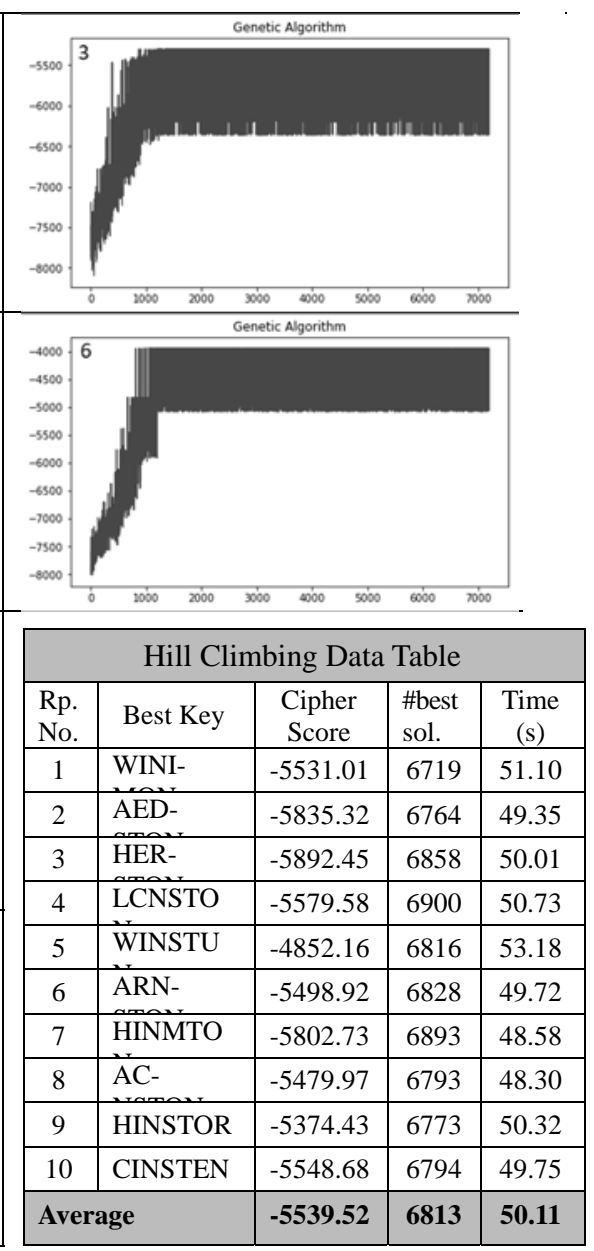

Table 3: Genetic Algorithm data.
Nevertheless, the scores that have been collected prove that this algorithm has the efficiency and reliability to find a solution to this problem in a small amount of time. The number of best solutions found throughout this process make this algorithm reliable.

Looking at the implementation of this algorithm the complexity is quadratic-time $\mathrm{O}\left(\mathrm{N}^{2}\right)$. This again suggests that this algorithm might be better suited to modeling shorter keys.

\subsection{Random Optimisation}

The random optimisation algorithm performs reasonably well for a random search, though it should be noted it is not as reliable as the other algorithms. The results in Table 4, demonstrate some reasonable results. It is also clear that it takes on average less time to complete and can get some good results. This can be shown by looking at the best keys, where some of the letters represent the original key used to encrypt: thus, it is quite close to finding the correct key. Not once though has the algorithm completed the process successfully. The data show that it has the potential to search a much wider scope if the number of repeats were increased in the algorithm. The best keys here could have the potential to uncover some small phrases, which could be useful to a cryptanalysis.

As mentioned previously, the inconsistency of this algorithm is what makes it weak, and ultimately unusable. On the other hand, when observing the graphs in Figure 3 , they all show an improving state; and one which represents the hill climbing event, where it tries to find a better solution to find its best result within the scope. Each repeats has a different climb and different number of best solutions found (as displayed in Figure 3). Interestingly, they all bare similar qualities of aiming for better solutions. 


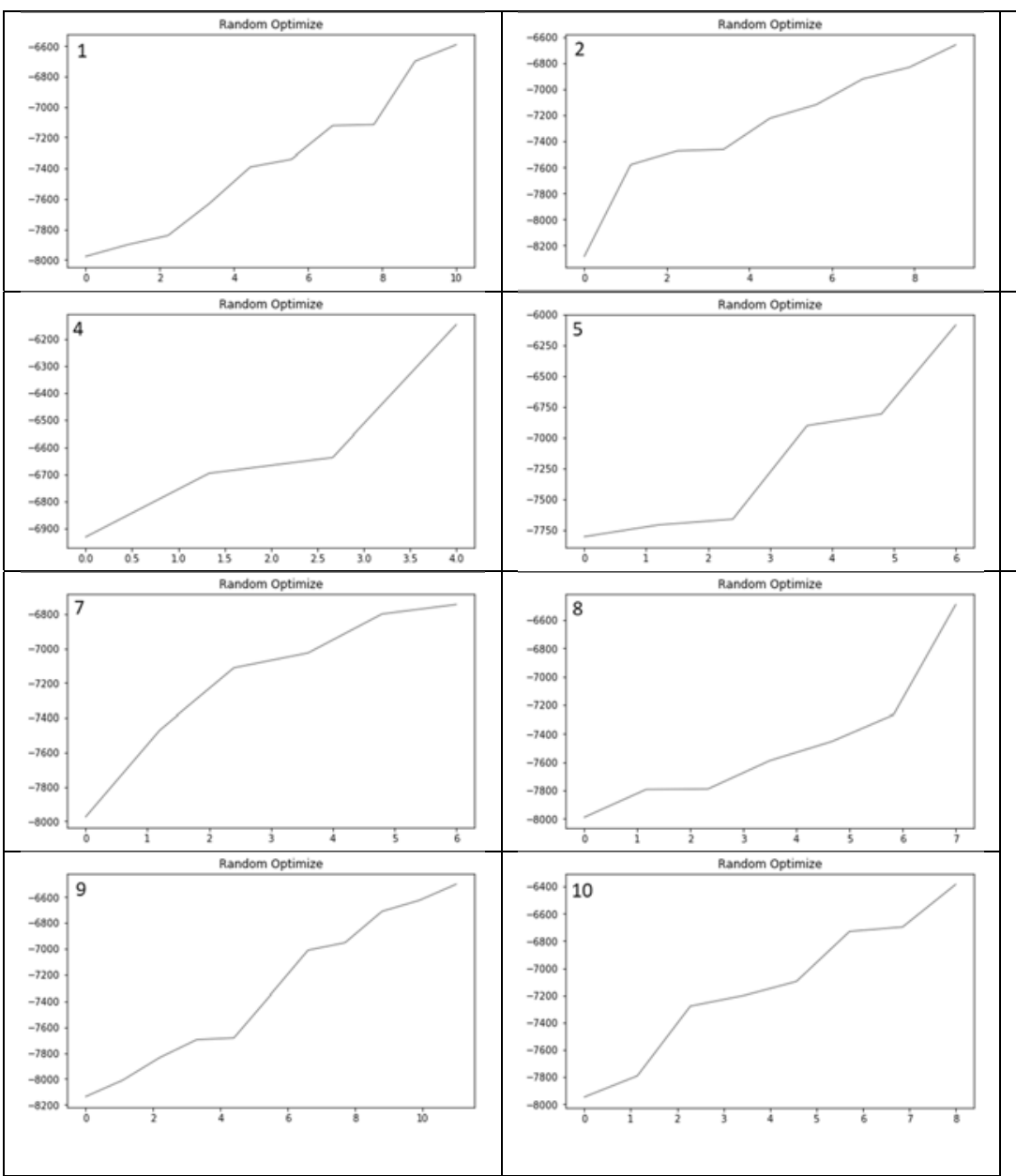

Figure 3: Random Optimisation graphs

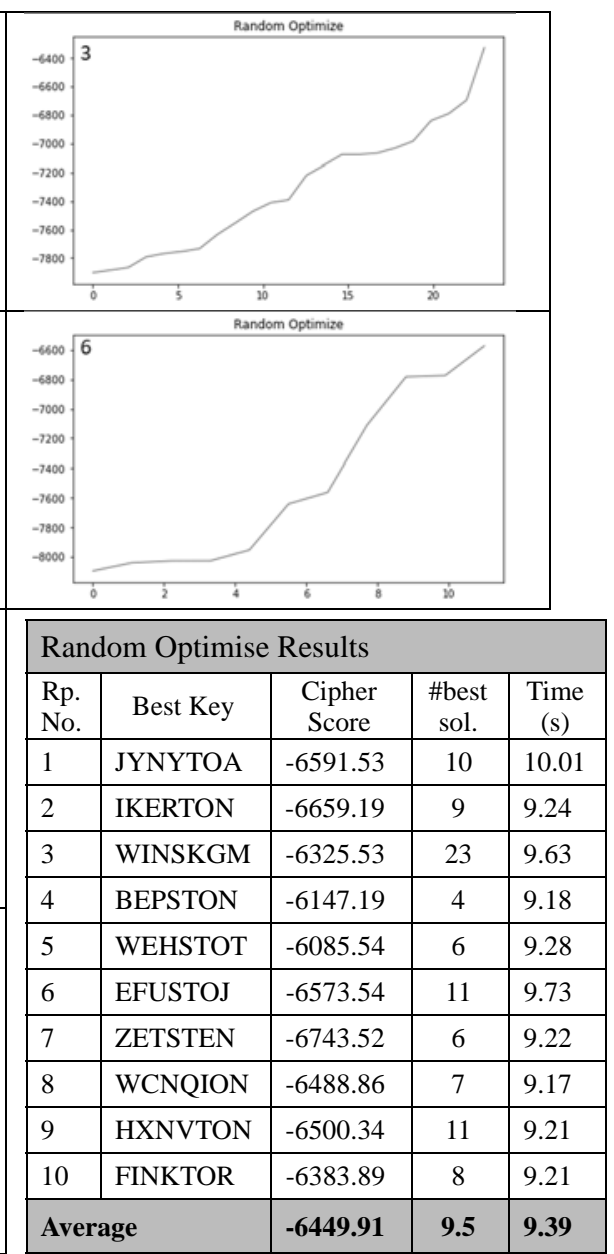

Table 4: Random Optimisation Results

(Y-axis: Score; X-axis: No. of Best Solutions Found).

However, they do this through different paths because of its randomness and unreliability. For smaller problems, this algorithm could be useful, although it has shown many weaknesses within this research; and for larger keys used, the chance of solving them is minimal.

Due to its simplicity the random optimisation algorithm has a complexity is of linear-time $\mathrm{O}(\mathrm{N})$ which means that for input (keys) there would be a proportional increase in the complexity and therefore the time taken.

\subsection{Simulated Annealing}

The simulated annealing algorithm struggles to model the keys, as shown in Table 5, all the best keys do not bare any resemblance to the key used (WINSTON) to encrypt the text. Simulated annealing does not produce any good results: the average cipher score implies that a text deciphered using a key with this score would result in a failure, since the message would still be unclear and not readable English. It is noteworthy that even though this algorithm was not successful it has the ability to find many different though weak solutions throughout the process in a reasonable amount of time.

The graphs in Figure 4 show an alternative view of the performance of the algorithm. Each have a bad start, with some results getting worse like repeats 6 . However, towards the end of the process the algorithms are now adjusting themselves to only accept better results. This is because the probability is less likely to accept the worse results; thus, it gradually ascends to a result that is better than the others. A good example of this can be shown in repeats 6 (Figure 4). It starts off unpredictable but at the end a curve appears where it begins to increase only using the conditions to accept better results. As it is shown in Figure 4, that all of the repeats exceed during the end of its process. 


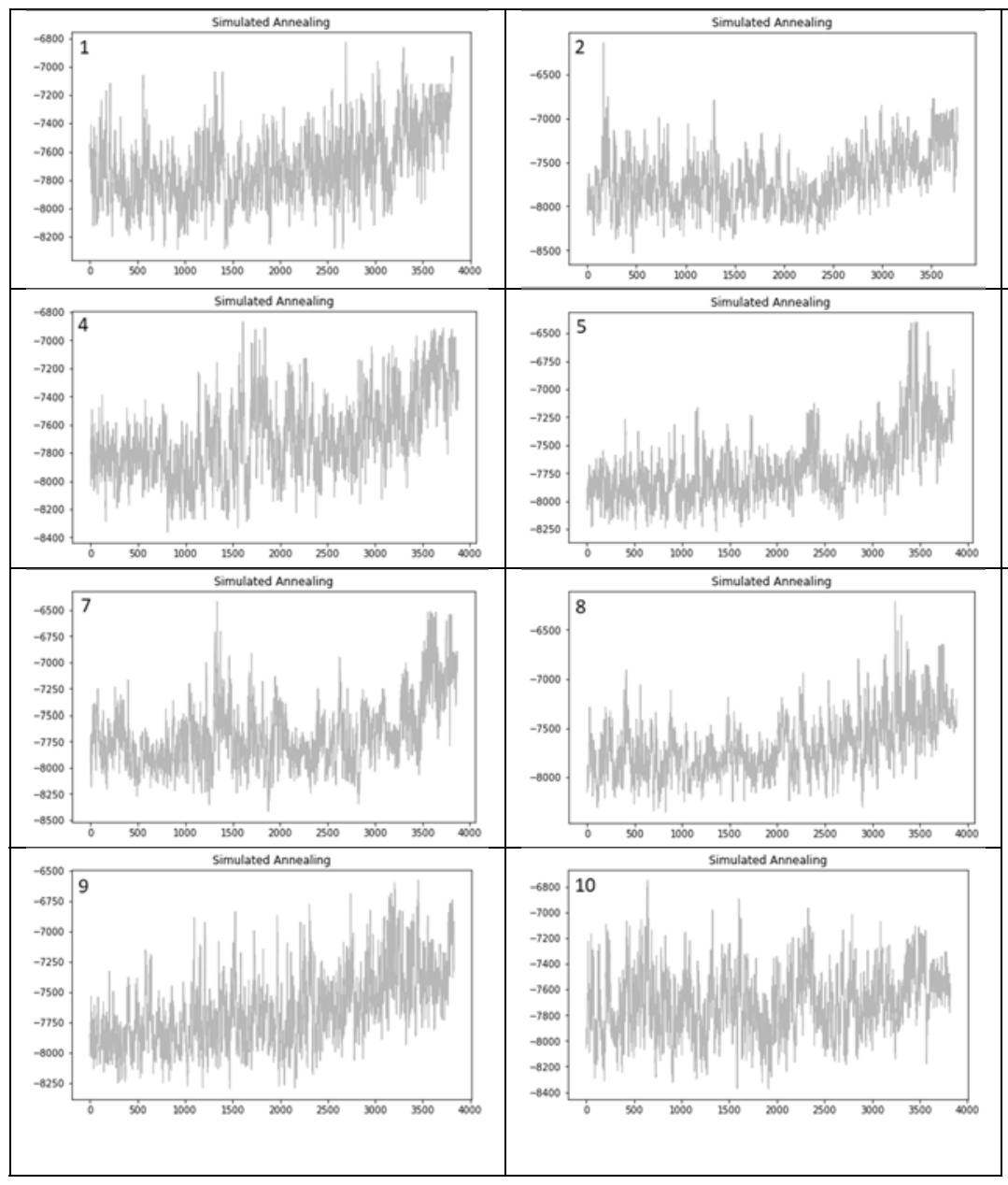

Figure 4: Simulated Annealing Graphs

(Y-axis: Score; X-axis: No. of Best Solutions Found).

In consequence, it can be suggested that with larger sized inputs this algorithm works a lot more efficiently in finding better solutions. Although, this is something that could be tested in further research in this area. The implementation of this algorithm has a complexity of $\mathrm{O}(\mathrm{N})$, this is linear-time, and implies that the increase in performance is dependent upon the size of the input given. Therefore, this could make this algorithm work more functionally with larger problems.

\subsection{Discussion}

Comparing the results for the algorithms tested as displayed in Figure 5, as well as analysing the results of all the tests, it has been shown that the genetic algorithm has been the most effective and efficient way for finding the correct solutions. most effective.

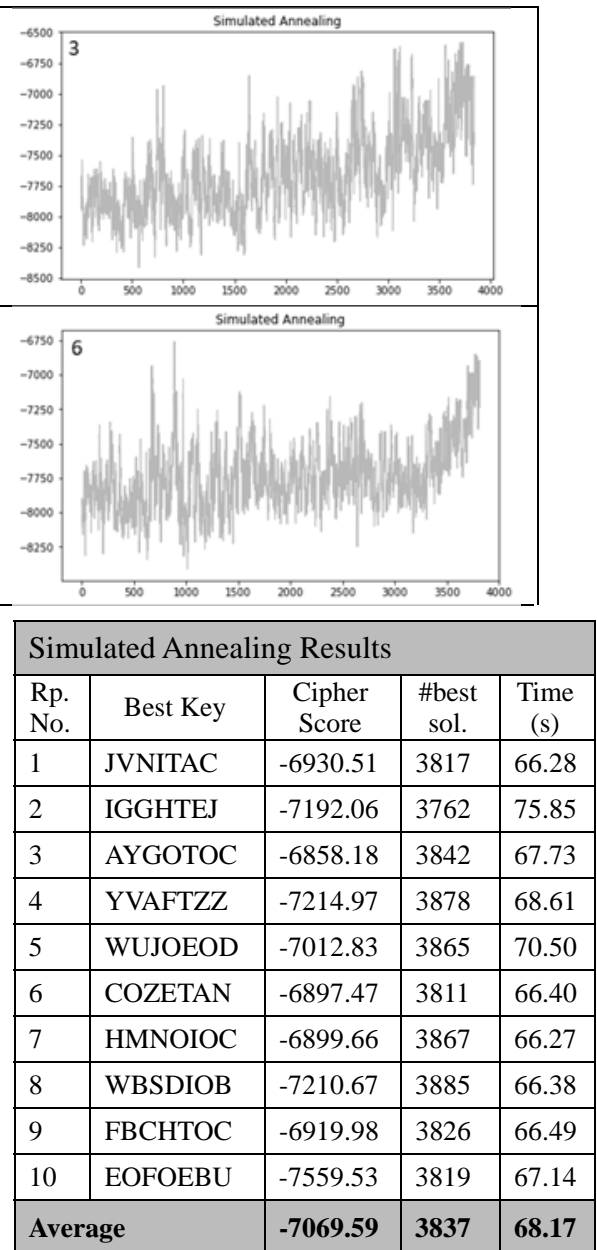

Table 5: Simulated Annealing Results

It has been the only algorithm to successfully find a correct solution. Finding the solution however is not the only factor and the time taken and number of best solutions found in the process are considered. Thus, due to all these contributors the genetic algorithm has been the

This is shown in Figure 5, as it illustrates that the genetic algorithm has nearly found the best solutions in under processing 1000 better solutions. On the contrary, the other algorithms are took longer to produce worst keys, and did not get the best result possible. However, it must be acknowledged that the hill climbing algorithm also performed well, nearly achieving the correct solution through widening the search scope. In this respect, it can also be considered a very effective algorithm and could be useful for discovering other solutions. 


\section{Conclusion}

This paper has investigated the use of machine learning algorithms for decrypting polyalphabetic substitution ciphers. Four well known machine learning algorithms were applied; hill climbing, genetic algorithms, simulated annealing and random optimisation.

It can be concluded that the genetic algorithm has been the most effective algorithm used in this research, with hill climbing as second. Furthermore, they both have the potential to be useful for larger problems. The main reason why genetic algorithm has performed best is that it has achieved the correct key used in three out of ten repeats, with an average time of 23.40 (s), and a consistent number of best solutions found.

It is possibly the nature by which the algorithm works that makes it so successful; however, this would be an ideal opportunity to exploit this algorithm further in extended research.

Throughout this work the polyalphabetic technique has been adopted for use of encryption and decryption. Using this difficult type of cipher demonstrates the power of the algorithms used, though some are clearly superior to others. While every effort was made to optimise the implementation of the machine learning algorithms, further improvements may be possible. Limited experimentation was undertaken to identify suitable training parameters and additional experimentation may yield improved results.

\section{References}

[1] Stamp M. Information security: Principles and practice. $2^{\text {nd }}$ edition. Oxford: Wiley, John \& Sons, 2011.

[2] Martin KM. Everyday cryptography: Fundamental principles and applications. Oxford: Oxford University Press, 2012.

[3] Schneier B. Applied cryptography, second edition. $2^{\text {nd }}$ ed. John Wiley \& Sons, 1996.

[4] Bergmann K. Cryptanalysis Using Nature-Inspired Optimization Algorithms. Master of Science. University of Calgary, 2007.

[5] Bell J. Machine learning. $1^{\text {st }}$ ed. Indianapolis, Indiana: John Wiley \& Sons, Inc., 2015.
Combined Results of random iteration

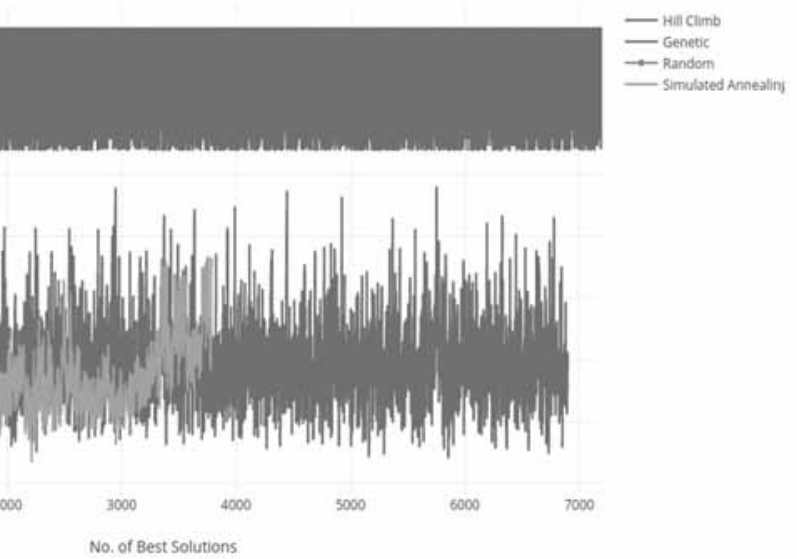

graphs for a single repeat of each technique.

[6] Zang H, Zhang S, Hapeshi K. A Review of Nature-Inspired Algorithms. Journal of Bionic Engineering, 7, pp. S232-S237, 2010.

[7] Russell S, Norvig P. Artificial intelligence. $3^{\text {rd }} \mathrm{ed}$. Pearson, pp.120-129, 2016.

[8] Lones M. Metaheuristics in nature-inspired algorithms. Proceedings of the 2014 conference companion on Genetic and evolutionary computation companion GECCO Comp '14, 2014.

[9] Practical Cryptography. Quadgram Statistics as a Fitness Measure. Available at: http://practicalcryptography.com/cryptanalysis/text-characterisation/quadgrams/\#a-python-implementation (Accessed: 13 February 2017), 2009.

[10] Segaran T. Programming collective intelligence: Building smart web 2.0 applications. United States: O'Reilly Media, Inc, USA, 2007.

[11] Bell R. A beginner's guide to Big O notation. Robbell.net (open access article). Available at: https://robbell.net/2009/06/a-beginners-guide-to-big-o-notation/ (Accessed: 12 April 2018), 2018.

[12] Conway D, White JM. Machine learning for hackers. Sudbury, MA, United States: O'Reilly Media, Inc, USA, 2012.

[13] Kahn D. The codebreakers: The comprehensive history of secret communication from ancient times to the Internet. New York, NY: Simon \& Schuster Adult Publishing Group 1997.

[14] Churchhouse RF. (2001) Codes and ciphers: Julius Caesar, the enigma and the internet. Cambridge: Cambridge University Press, 2001.

[15] Gollmann D. Computer security. $3^{\text {rd }}$ ed. Chichester, United Kingdom: Wiley, John \& Sons, 2010. 


\title{
Generating of Task-Based Controls for Joint-Arm Robots with Simulation-based Reinforcement Learning
}

\author{
Georg Kunert* , Thorsten Pawletta \\ Wismar University of Applied Sciences, Fac. of Engineering, Research Group CEA, \\ Postfach 1210, 23952 Wismar, Germany; georg.kunert@cea-wismar.de
}

SNE 28(4), 2018, 149 - 156, DOI: 10.11128/sne.28.tn.10442 Received: October 25, 2018; Revised November 15, 2018; Accepted November 24, 2018

SNE - Simulation Notes Europe, ARGESIM Publisher Vienna ISSN Print 2305-9974, Online 2306-0271, www.sne-journal.org

Abstract. The paper investigates how a robot control for a pick-and-place application can be learned by simulation using the Q-Learning method, a special Reinforcement Learning approach. Furthermore, a post-optimization approach to improve a learned strategy is presented. Finally, it is shown how the post-optimized strategy can be automatically transformed into an executable control using the simulation-based control approach.

\section{Introduction}

The conventional robot oriented programming focuses on a special type of robot and on one explicit control problem. That means, one special program is developed to solve one problem. Typical programming approaches according to [1] are: (i) offline programming with a specific robot programming (RP) language, (ii) teach-in procedures, (iii) master-slave procedures and (iv) play-back procedures. In conjunction with the RP there are different manufacturer-specific and non-proprietary ComputerAided-Robotics (CAR) systems, such as KUKA-Sim or EASY-ROB.

Another approach is the specification of the Robot Operating System (ROS) - Industrial for robot controller, which was driven by the administration of the ROS consortium. ROS-Industrial defines a layer model and an interface standard. The layer model supports the integration of CAR systems and the application of different abstract programming approaches.
One abstraction approach established in robotics is the task-oriented programming. Using this approach, tasks that are specified are robot independent. A transformation method is required to execute the task-based control.

Already before ROS-Industrial was launched, the Research Group Computational Engineering and Automation (RG CEA) at Hochschule Wismar started the development of the open Robotic Control and Visualization (RCV) Toolbox for MATLAB (RCV Tbx., www.ceawismar.de, accessed 05/2018; [3], which supports manufacturer-independent control development.

The RCV toolbox provides a set of abstract robot-oriented commands in MATLAB in analogy to an RP language and a robot control can be developed virtually. In addition, the RG CEA proposed a specific framework and procedure model, called Simulation Based Control (SBC) approach [4]. Robot controls can be developed type independent, task-oriented, and model-based using the SBC approach in conjunction with the use of ROSIndustrial or the RCV toolbox.

The usage of a Reinforcement Learning (RL) method in conjunction with a simulated process model to develop a real robot control is investigated in this paper. The approach is illustrated by a typical pick-and-place problem (PP). The PP problem is mapped to a simulation model according to the requirements of the learning algorithm. The control strategy is learned offline using the RL method and then transformed into an executable robot control using the SBC approach. This research is seen as a first step to learn and generate executable robot controls using the SBC approach and integrated learning methods for whole problem classes, such as the class of assembly problems. 
The basics of RL and a special approach, the QLearning method [5, 6], are explained with the help of an example at the beginning. Thereafter, it is pointed out how a learned behavioral strategy can be improved by a post-optimization. Then it is shown, how a learned and post-optimized strategy can be automatically transformed into an executable robot control using the SCB approach. Finally, an outlook on future work is given.

\section{Reinforcement Learning}

Besides the supervised and unsupervised learning, the RL forms an independent group of learning methods [6]. The aim of RL is to learn a behavior strategy to propose actions to reach a given target state.

The advantage of this learning approach is that no prior knowledge in the form of training data and no teacher is needed. An RL method is training itself by the trial-and-error principle.

\subsection{Basics of Reinforcement Learning}

Figure 1 shows the basic structure of the RL approach. An RL method comprises the environment and at least one agent.

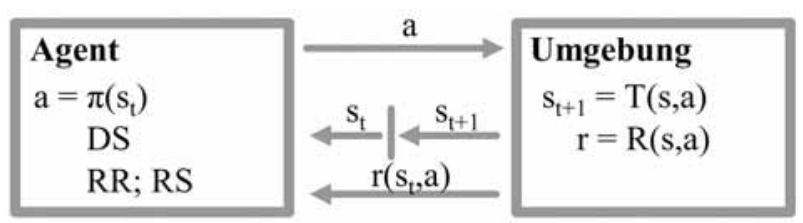

Figure 1: Basic structure of the RL approach.

The environment can enter a finite number of states $s \epsilon S$. The environment communicates its start state $s_{t}$ to the agent. The agent then reacts with an action $a$. Based on the action $a$ of the agent, the environment sends a reward $r$ and shifts into a following state $s_{t+1}$. The reward $r$ is a feedback on the action $a$ of the agent. The interaction between agent and environment is repeated until the environment reaches a successful or unsuccessful target state $s_{\text {target }} \epsilon S$. In RL, this iteration until the environment reaches a target state is called an episode.

For Markov-processes of first order the environment generates a reward according to Equation 1 and calculates the following state $s_{t+1} \epsilon S$ according to Equation 2 .

$$
\begin{gathered}
r=R\left(s_{t}, a\right) \\
s_{t+1}=T\left(s_{t}, a\right)
\end{gathered}
$$

The reward model $R$ and the state transition model $T$ are functions of the current state of the environment und the current action of the agent. The following state can also be identical to the state before. The reward is a scalar value. The range of the rewards of the environment is often given by $r \in\{-\infty,-1,0,+1\}$. A negative infinity means that the action of the agent was not allowed.

A minus one means that the environment is in an unsuccessful target state. If the environment is in a successful target state, the reward is plus one. Actions that are rewarded with a zero, are allowed but did not lead to a target state. These states are states between start state and target state. The agent saves all states $s \in S$ he receives from the environment in a data structure $D S$ (see Figure 5). In this way he collects knowledge about the environment. Through the ability of saving states, the agent can identify states that he has previously explored. Other parts of the agent as shown in Figure 1 are described later. $Q$ is explained in Section 1.2, while $R R$ and $R S$ are explained in Section 3.

The training phase of the RL consists of a multitude of episodes. At the beginning, the agent explores the environment solely using the trial-and-error method. In later episodes, the actions of the agent are evaluated based on the collected knowledge using a learning method.

An often used learning method is the Q-Learning. The objective is to reach the target state with as little as possible numbers of state changes of the environment. The result of the learning phase is a learned behavior strategy $\pi$ of the agent. According to Equation 3, $\pi$ is a function of the current state of the environment and returns a target-oriented action $a$.

$$
a=\pi\left(s_{t}\right)
$$

While learning, the agent is in the so called explorationexploitation-dilemma. Exploitation means learning based on the acquired knowledge and exploration means the study of unknown strategies by trying random actions.

Often, the RL needs many episodes for a successful iterative learning. The computing time depends significantly on the complexity of the state space and the reaction time of the environment. Due to the reaction time, the possibility of learning in the real environment is often not practicable for robotic applications. Therefore, the behavior of the environment is simulated using a model and learning takes place offline. 


\subsection{Q-Learning}

As described before, the goal of the RL is to learn the best possible behavioral strategy $\pi$ according to Equation 3 to achieve - in the simplest form - a target state.

Q-Learning is a special feature of RL for solving optimization problems. It is based on the idea of learning with the help of a memory matrix $W\left(a, s_{t}\right)$, called Q-Matrix for Q-Learning, as introduced by Watkins [7]. The method of Q-Learning exists in different forms [5, 6]. In the following, the approach of value based learning (VBL) will be briefly introduced. According to Akh$\operatorname{tar}$ [6], VBL represents a special approach to Q-Learning.

With VBL, the agent does not have an internal model of the environment at the beginning. He initially investigates by purely random actions $a \epsilon A$ the states $s \in S$ as well as rewards $r$ of the environment and learns, based on this information, iteratively his internal model of the environment, the so-called Q-function $Q\left(s_{t}, a\right)$. The Q-function describes the expected benefit, the Q-value, of an action $a$ in the state $s_{t}$. The Q-function is mapped inside the agent using a dynamically growing matrix called Q-Matrix. Each row of the Q-Matrix represents an explored state $s \epsilon S$ of the environment and each column represents a possible action $a \epsilon A$ of the agent. That means that the Q-Matrix stores the iteratively learned knowledge of the agent.

At the beginning of the training phase, that means at the start of the first episode, the Q-Matrix is a $0 \times n$ matrix. The column dimension corresponds to the number of possible actions. For each newly investigated state $s \in S$ the Q-Matrix is extended by one row and all elements of the new row are initialized with 0 . The calculation of the Q-value of a current state-action-pair $\left(s_{t}, a\right)$ can be fulfilled in several ways. Equation 4 shows a simple Q-Learning approach.

$$
Q\left(s_{t}, a\right)=r\left(s_{t}, a\right)+\gamma \cdot \max \left(\mathrm{Q}\left(s_{t+1}, \forall a \epsilon A\right)\right)
$$

As described in the previous subsection, the environment responds to an action $a$ of the agent with a following state $s_{t+1}$ and a reward $r$ according to Equation 1 and Equation 2. The benefit $Q\left(s_{t}, a\right)$ is given by Equation 4 from the sum of the current reward of the state-action-pair $\left(s_{t}, a\right)$ and the weighted maximum benefit of all follow-up actions. The latter is calculated from the previously established Q-Matrix values. For this purpose, all columns $\forall a \in A$ in the row of the following state $s_{t+l}$ of the Q-Matrix are analyzed.
The weighting factor $\gamma$ is called the discounting factor. It ranges between 0 and 1 and controls the impact of expected future rewards on the current decision.

Extended Q-Learning methods additionally introduce a learning rate parameter $\alpha$. They have the possibility of weighting between the already learned value $Q\left(s_{t}, a\right)$, the current reward and the expected future rewards $[5,8]$.

Regardless of the specific Q-Learning method, a large number of episodes are executed in the training phase. An episode starts with a random or known initial state $s$ $\epsilon S$ of the environment. Subsequently, in each episode randomly (exploration) or on the basis of already known Q-values (exploitation) actions $a \in A$ are tried on the environment in order to improve the internal model of the agent in the form of Q-values. An episode ends when a target state of the environment has been reached. The agent recognizes this by evaluating the returned reward value. An additional parameter may be used for an exploration-exploitation-weighting. This feature can be used for example to decrease the exploration rate as the number of episodes increases.

After the training phase the Q-Matrix represents the learned behavior strategy $\pi$. Therefore, in the case of QLearning, Equation 3 can be concretized as follows.

$$
\pi\left(s_{t}\right)=\operatorname{argmax}\left(\mathrm{Q}\left(s_{t}, \forall a \in A\right)\right)
$$

The agent gets the initial state from the environment as the current state $s_{t}$. According to Equation 5, the agent looks in the row associated with the current state $s_{t}$ in the Q-Matrix for the maximum Q-value and determines the argument of the column. This represents the best action $a$ in the state $s_{t}$. The determined action $a$ is executed and the following state $s_{t+1}$ is received. Thereafter, $s_{t+l}$ is regarded as the current state $s_{t}$ and the next action $a$ according to Equation 5 is determined. The iteration is continued until the following state $s_{t+1}$ transmitted by the environment corresponds to a target state. The agent detects the end of the episode by evaluating the reward value $r$. In this case, rows of the Q-Matrix that represent a target state remain zero, because the agent does not execute any actions after reaching the target state.

The Q-Learning approach is based on a backpropagation algorithm. It is not certain that the learned strategy is optimal, because it is not sure, that the shortest state sequence has been found from a start state to the target state. This problem is discussed in more detail in Section 3. Before, Q-Learning will be illustrated with an example in the next section. 


\section{Application Example}

As an application example, the game Towers of Hanoi (ToH), a single player application, has been chosen. This corresponds to a typical pick-and-place application for joint-arm robots. The rules of the game ToH are simple and will be explained in the following.

There are three bars. On one bar an arbitrary number of different sized discs are stacked on each other. They are sorted by size and start at the bottom with the largest disc. The aim of the game is to relocate the discs to another bar, whereby only one disc has to be moved per turn. Furthermore, it is not allowed to put a larger on a smaller disc. The storage options are defined by the bars. For the purposes of the RL, the bars and discs form the environment and the player, later the robot, is the agent. The states of the environment result from the positions of the disks on the bars. The movement of discs are actions of the agent that lead to state transitions of the environment. Figure 3 shows all possible states of a two-disc game.

The rules of the game are to be modeled in state transition model $T$ of the environment. According to Equation 2, $T$ generates the following state $s_{t+1}$ as a reaction on an action $a$. If an action $a$ was not allowed, the following state $s_{t+l}$ returned by $T$ remains the current state $s_{t}$.

Figure 3 shows the state transition diagram for an environment with two discs. The numbering of the states corresponds to the numbering in Figure 2. Furthermore, a reward model $R$ according to Equation 1 is specified in the environment.

The rules of the game provide rewards $r \epsilon\{0,1,-\infty\}$. The reward $r=1$ represents, that the target state $s_{9}$ as depicted in Figure 2 has been reached.

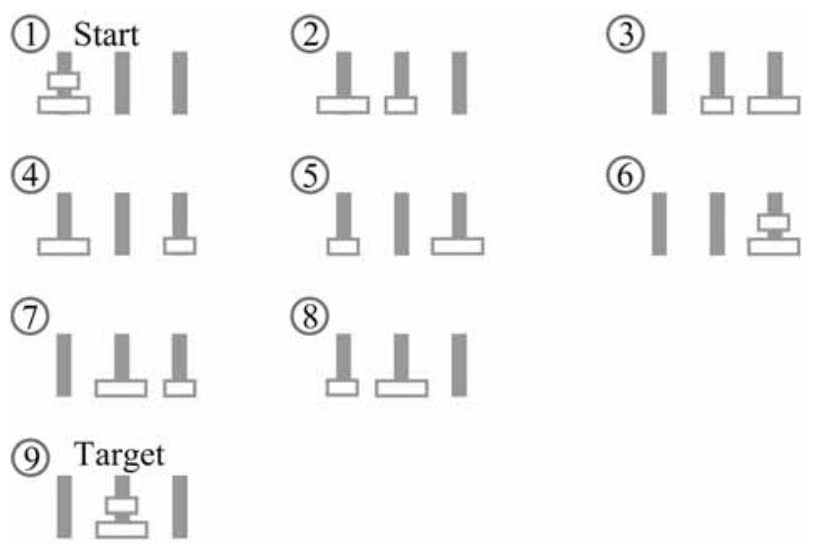

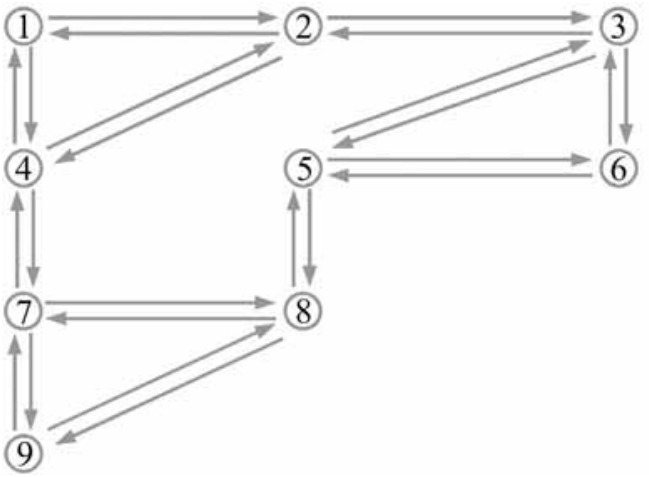

Figure 3: State transition diagram for $\mathrm{ToH}$ with two discs.

Illegal actions $a$ receive the reward $r=-\infty$. The reward $r=0$ stands for reaching a following state $s_{t+1}$, which is not a target state.

A reward $r=-1$ is not used here because there is no target state that is considered to be a failure. Moving a disc to another bar is an action $a$. As shown in Figure 4, the agent at $\mathrm{ToH}$ always has a choice of six actions. The restriction of the action set $A$ to these six actions implies that: (i) a picked up disk can only be placed on one of the other two bars and (ii) only one disk can be taken at a time.

From the action set $A$ shown in Figure 4, one can derive that the Q-Matrix of the agent has the column dimension of six. The number of rows grows dynamically during learning. At the start of the training phase no state is known for the agent.

Figure 2 shows the fixed initial state $s_{l}$, for ToH. This state is communicated to the agent as the first state $s_{l}$ of the environment.

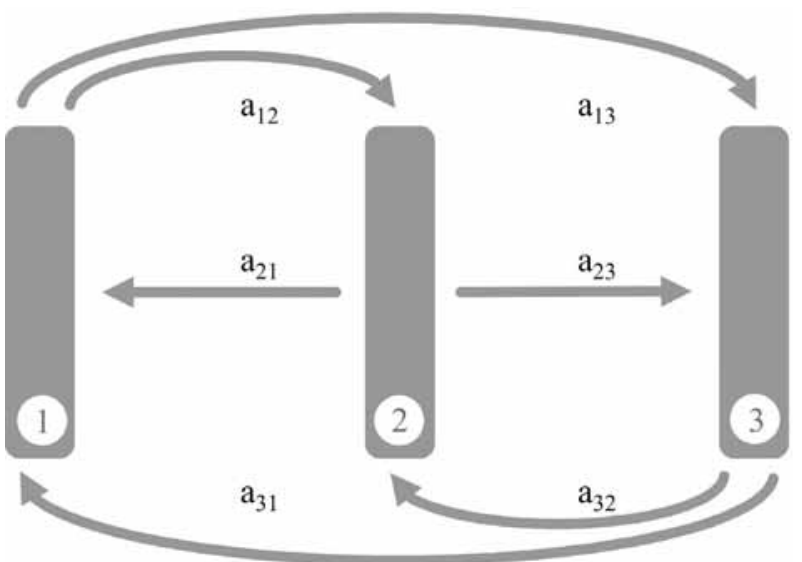

Figure 4: Actions set $\mathrm{A}$ of the agent at $\mathrm{ToH}$.

Figure 2: All states of ToH with two disks. 
Each state $s_{t}$ is characterized by the positions of the two discs on the three bars, which can be mapped to six attributes. The current values of the state attributes are stored in the agent in the data structure $D S$, as shown in Figure 5. The attribute values have the following interpretation: 0 no disc; 1 small disc; 2 big disc.

The first element (attribute) of each line describes the existence or size of the upper disk of the first bar and the second the existence or size of the lower disk of the first bar. The third to sixth elements of each row similarly describe the states of the second and third bars.

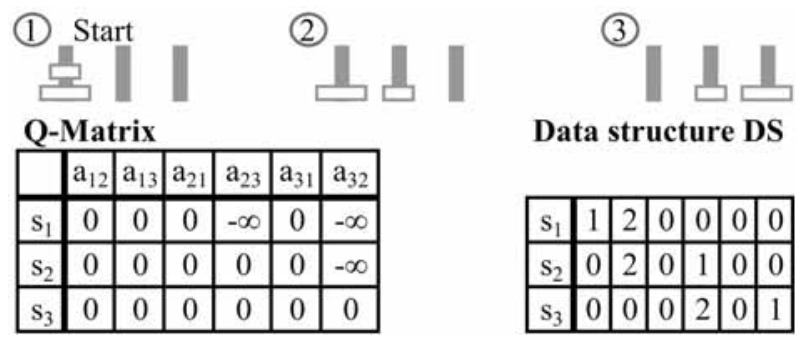

Figure 5: Possible development of the Q-Matrix and the data structure DS of the agent at beginning of the training phase.

During the training phase, each following state $s_{t+1} \in S$ communicated by the environment is analyzed in the agent. If the state is not yet contained in $D S$, then: (i) the state is stored in $D S$ and (ii) the Q-Matrix is extended by one row in which all elements are zero. If the state was already known or was newly recorded, then according to Equation 4 the utility $Q$ of the last state action pair $\left(s_{t}, a\right)$ is calculated.

For this, the reward $r$ communicated by the environment is evaluated. In this example, a discount factor $\gamma$ of 0.8 was chosen. As shown in Figure 5, at the beginning of the training phase, often only new states are recorded. Thus, new states are building up the Q-Matrix with zero elements. The iterative learning of the utility values $Q\left(s_{t}, a\right)$ takes place in subsequent episodes.

Figure 6 shows examples of Q-Matrices calculated in different episodes. Ideally, in the first episode, all states $s \in S$ of the environment are explored by the agent. As shown in Figure 6, the Q-Matrix then has the row dimension nine.

After completion of episode 1, the Q-Matrix further shows that the state-action-pair $\left(s_{8}, a_{1}\right)$ with utility value 1 leads to the target state (see Figure 2 and Figure 4). The QMatrix of episode 2 shows a possible learning progress.
Episode 1

\begin{tabular}{|c|c|c|c|c|c|c|}
\hline & $\mathrm{a}_{12}$ & $\mathrm{a}_{13}$ & $\mathrm{a}_{21}$ & $\mathrm{a}_{23}$ & $\mathrm{a}_{31}$ & $\mathrm{a}_{32}$ \\
\hline $\mathrm{s}_{1}$ & 0 & 0 & $-\infty$ & $-\infty$ & $-\infty$ & $-\infty$ \\
\hline $\mathrm{s}_{2}$ & 0 & 0 & 0 & 0 & 0 & $-\infty$ \\
\hline $\mathrm{s}_{3}$ & 0 & 0 & 0 & 0 & 0 & $-\infty$ \\
\hline $\mathrm{s}_{4}$ & 0 & $-\infty$ & $-\infty$ & 0 & 0 & 0 \\
\hline $\mathrm{s}_{5}$ & 0 & 0 & 0 & 0 & 0 & 0 \\
\hline $\mathrm{s}_{6}$ & $-\infty$ & 0 & 0 & 0 & 0 & 0 \\
\hline $\mathrm{s}_{7}$ & $-\infty$ & 0 & 0 & $-\infty$ & 0 & 0 \\
\hline $\mathrm{s}_{8}$ & 1 & 0 & $-\infty$ & 0 & 0 & 0 \\
\hline $\mathrm{s}_{9}$ & 0 & 0 & 0 & 0 & 0 & 0 \\
\hline
\end{tabular}

$$
\begin{aligned}
& \mathrm{Q}\left(\mathrm{s}_{8}, \mathrm{a}_{1}\right)=\mathrm{r}\left(\mathrm{s}_{8}, \mathrm{a}_{1}\right)+ \\
& \gamma \cdot \max \left(\mathrm{Q}\left(\mathrm{s}_{9}, \forall \mathrm{a} \in \mathrm{A}\right)\right)
\end{aligned}
$$

$$
1=1+\gamma \cdot 0
$$

Episode 2
\begin{tabular}{|c|c|c|c|c|c|c|}
\hline & $\mathrm{a}_{12}$ & $\mathrm{a}_{13}$ & $\mathrm{a}_{21}$ & $\mathrm{a}_{23}$ & $\mathrm{a}_{31}$ & $\mathrm{a}_{32}$ \\
\hline $\mathrm{s}_{1}$ & 0 & 0 & $-\infty$ & $-\infty$ & $-\infty$ & $-\infty$ \\
\hline $\mathrm{s}_{2}$ & $-\infty$ & 0 & 0 & 0 & 0 & $-\infty$ \\
\hline $\mathrm{s}_{3}$ & 0 & 0 & 0 & 0 & 0 & $-\infty$ \\
\hline $\mathrm{s}_{4}$ & 0 & $-\infty$ & $-\infty$ & 0 & 0 & 0 \\
\hline $\mathrm{s}_{5}$ & 0 & 0 & $-\infty$ & 0 & 0 & 0,8 \\
\hline $\mathrm{s}_{6}$ & $-\infty$ & 0 & $-\infty$ & $-\infty$ & 0 & 0 \\
\hline $\mathrm{s}_{7}$ & $-\infty$ & 0 & 0 & $-\infty$ & 0 & 0 \\
\hline $\mathrm{s}_{8}$ & 1 & 0 & $-\infty$ & 0 & 0 & $-\infty$ \\
\hline $\mathrm{s}_{9}$ & 0 & 0 & 0 & 0 & 0 & 0 \\
\hline
\end{tabular}

$$
\begin{aligned}
& \mathrm{Q}\left(\mathrm{s}_{5}, \mathrm{a}_{5}\right)=\mathrm{r}\left(\mathrm{s}_{5}, \mathrm{a}_{5}\right)+ \\
& \gamma \cdot \max \left(\mathrm{Q}\left(\mathrm{s}_{8}, \forall \mathrm{a} \in \mathrm{A}\right)\right)
\end{aligned}
$$

$$
0,8=0+\gamma \cdot 1
$$

Episode n

\begin{tabular}{|c|c|c|c|c|c|c|}
\hline & $\mathrm{a}_{12}$ & $\mathrm{a}_{13}$ & $\mathrm{a}_{21}$ & $\mathrm{a}_{23}$ & $\mathrm{a}_{31}$ & $\mathrm{a}_{32}$ \\
\hline $\mathrm{s}_{1}$ & 0,41 & 0 & $-\infty$ & $-\infty$ & $-\infty$ & $-\infty$ \\
\hline $\mathrm{s}_{2}$ & $-\infty$ & 0,51 & 0 & 0 & $-\infty$ & $-\infty$ \\
\hline $\mathrm{s}_{3}$ & $-\infty$ & $-\infty$ & 0,64 & 0 & 0 & $-\infty$ \\
\hline $\mathrm{s}_{4}$ & 0 & $-\infty$ & $-\infty$ & $-\infty$ & 0 & 0 \\
\hline $\mathrm{s}_{5}$ & 0 & 0 & $-\infty$ & $-\infty$ & $-\infty$ & 0,8 \\
\hline $\mathrm{s}_{6}$ & $-\infty$ & $-\infty$ & $-\infty$ & $-\infty$ & 0 & 0 \\
\hline $\mathrm{s}_{7}$ & $-\infty$ & $-\infty$ & 0 & $-\infty$ & 0 & 1 \\
\hline $\mathrm{s}_{8}$ & 1 & 0 & $-\infty$ & 0 & $-\infty$ & $-\infty$ \\
\hline $\mathrm{s}_{9}$ & 0 & 0 & 0 & 0 & 0 & 0 \\
\hline
\end{tabular}

Figure 6: Possible development of the Q-Matrix during the training phase.

Due to the backpropagation algorithm, the learning phase in ToH is completed, when an element of the first row of the Q-Matrix has a value greater than zero. This case is shown in the Q-Matrix of episode $n$.

After the training phase the learned behavioral strategy $\pi$ is derived from the Q-Matrix according to Equation 5. The example in Figure 7 shows the generation of actions based on $\pi$. $E$ stands for the environment and $A g$ for the agent. The example refers to the states $s \in S$ in Figure 2, the actions $a \epsilon A$ in Figure 4, and the Q-Matrix of episode $n$ in Figure 6.

From the example results the sequence of state-action-pairs:

$\left[\left(\mathrm{s}_{1}, \mathrm{a}_{12}\right),\left(\mathrm{s}_{2}, \mathrm{a}_{13}\right),\left(\mathrm{s}_{3}, \mathrm{a}_{21}\right),\left(\mathrm{s}_{5}, \mathrm{a}_{32}\right),\left(\mathrm{s}_{8}, \mathrm{a}_{12}\right),\left(\mathrm{s}_{9}\right.\right.$, cancel $\left.)\right]$. A comparison with the state transition diagram in Figure 3 shows that no optimal $\pi$ was learned, because the shortest 'path' from the start to the target state was not found. 


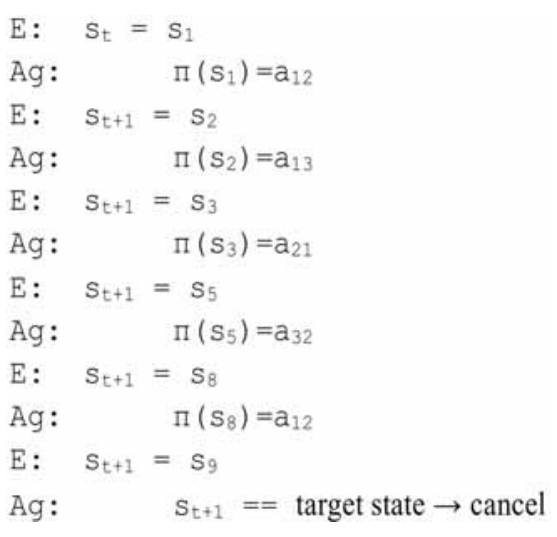

Figure 7: Example for the generation of actions based on $\pi$.

\section{Post-Optimization of the Behavior Strategy}

The goal of post-optimization is to search for a shorter sequence of state-action-pairs that map the shortest path from the start to the target state (Figure 3). For this it is reasonable to save all rewards $r$ and following states $s_{t+1}$ communicated by the environment in the agent during the training phase. For this purpose, two more matrices $R R$ (Received-Reward) and $R S$ (Received-followingState) are set up dynamically in the agent (Figure 1). Figure 8 shows the $R S$ and $R R$ matrices of the considered ToH example. Their structure corresponds to the Q-Matrix. The row index represents the states explored by the agent and the columns correspond to the action set $A$. In $R S$, as in the example of $\mathrm{ToH}$, a deterministic behavior of the environment is assumed.

The post-optimization is based on an iterative recalculation of the Q-Matrix using the knowledge stored in the matrices $R R$ and $R S$. Listing 1 shows the algorithm as pseudocode and Figure 9 shows the resulting Q-Matrix after the first, second and last (ninth) iteration.

\section{RR-Matrix}

\begin{tabular}{|c|c|c|c|c|c|c|}
\hline & $\mathrm{a}_{12}$ & $\mathrm{a}_{13}$ & $\mathrm{a}_{21}$ & $\mathrm{a}_{23}$ & $\mathrm{a}_{31}$ & $\mathrm{a}_{32}$ \\
\hline $\mathrm{s}_{1}$ & 0 & 0 & $-\infty$ & $-\infty$ & $-\infty$ & $-\infty$ \\
\hline $\mathrm{s}_{2}$ & $-\infty$ & 0 & 0 & 0 & $-\infty$ & $-\infty$ \\
\hline $\mathrm{s}_{3}$ & $-\infty$ & $-\infty$ & 0 & 0 & 0 & $-\infty$ \\
\hline $\mathrm{s}_{4}$ & 0 & $-\infty$ & $-\infty$ & $-\infty$ & 0 & 0 \\
\hline $\mathrm{s}_{5}$ & 0 & 0 & $-\infty$ & $-\infty$ & $-\infty$ & 0 \\
\hline $\mathrm{s}_{6}$ & $-\infty$ & $-\infty$ & $-\infty$ & $-\infty$ & 0 & 0 \\
\hline $\mathrm{s}_{7}$ & $-\infty$ & $-\infty$ & 0 & $-\infty$ & 0 & 1 \\
\hline $\mathrm{s}_{8}$ & 1 & 0 & $-\infty$ & 0 & $-\infty$ & $-\infty$ \\
\hline $\mathrm{s}_{9}$ & 0 & 0 & 0 & 0 & 0 & 0 \\
\hline
\end{tabular}

\section{RS-Matrix}

\begin{tabular}{|c|c|c|c|c|c|c|}
\hline & $\mathrm{a}_{12}$ & $\mathrm{a}_{13}$ & $\mathrm{a}_{21}$ & $\mathrm{a}_{23}$ & $\mathrm{a}_{31}$ & $\mathrm{a}_{32}$ \\
\hline $\mathrm{s}_{1}$ & 2 & 4 & 1 & 1 & 1 & 1 \\
\hline $\mathrm{s}_{2}$ & 2 & 3 & 1 & 4 & 2 & 2 \\
\hline $\mathrm{s}_{3}$ & 3 & 3 & 5 & 6 & 2 & 3 \\
\hline $\mathrm{s}_{4}$ & 7 & 4 & 4 & 4 & 1 & 2 \\
\hline $\mathrm{s}_{5}$ & 3 & 6 & 5 & 5 & 5 & 8 \\
\hline $\mathrm{s}_{6}$ & 6 & 6 & 6 & 6 & 5 & 3 \\
\hline $\mathrm{s}_{7}$ & 7 & 7 & 4 & 7 & 8 & 9 \\
\hline $\mathrm{s}_{8}$ & 9 & 7 & 8 & 5 & 8 & 8 \\
\hline $\mathrm{s}_{9}$ & 0 & 0 & 0 & 0 & 0 & 0 \\
\hline
\end{tabular}

Figure 8: RR- and RS-Matrix after the training phase.

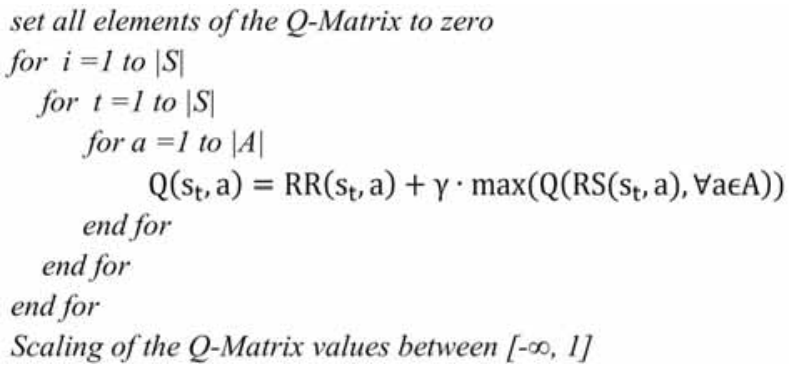

Listing 1: Pseudocode of the post-optimization algorithm.

\section{Q-Matrix - 1. Iteration}

Q-Matrix - 2. Iteration

\begin{tabular}{|c|c|c|c|c|c|c|}
\hline & $\mathrm{a}_{12}$ & $\mathrm{a}_{13}$ & $\mathrm{a}_{21}$ & $\mathrm{a}_{23}$ & $\mathrm{a}_{31}$ & $\mathrm{a}_{32}$ \\
\hline $\mathrm{s}_{1}$ & 0 & 0 & $-\infty$ & $-\infty$ & $-\infty$ & $-\infty$ \\
\hline $\mathrm{s}_{2}$ & $-\infty$ & 0 & 0 & 0 & $-\infty$ & $-\infty$ \\
\hline $\mathrm{s}_{3}$ & $-\infty$ & $-\infty$ & 0 & 0 & 0 & $-\infty$ \\
\hline $\mathrm{s}_{4}$ & 0 & $-\infty$ & $-\infty$ & $-\infty$ & 0 & 0 \\
\hline $\mathrm{s}_{5}$ & 0 & 0 & $-\infty$ & $-\infty$ & $-\infty$ & 0 \\
\hline $\mathrm{s}_{6}$ & $-\infty$ & $-\infty$ & $-\infty$ & $-\infty$ & 0 & 0 \\
\hline $\mathrm{s}_{7}$ & $-\infty$ & $-\infty$ & 0 & $-\infty$ & 0 & 1 \\
\hline $\mathrm{s}_{8}$ & 1 & 0,8 & $-\infty$ & 0 & $-\infty$ & $-\infty$ \\
\hline $\mathrm{s}_{9}$ & 0 & 0 & 0 & 0 & 0 & 0 \\
\hline
\end{tabular}

\begin{tabular}{|c|c|c|c|c|c|c|}
\hline & $\mathrm{a}_{12}$ & $\mathrm{a}_{13}$ & $\mathrm{a}_{21}$ & $\mathrm{a}_{23}$ & $\mathrm{a}_{31}$ & $\mathrm{a}_{32}$ \\
\hline $\mathrm{s}_{1}$ & 0 & 0 & $-\infty$ & $-\infty$ & $-\infty$ & $-\infty$ \\
\hline $\mathrm{s}_{2}$ & $-\infty$ & 0 & 0 & 0 & $-\infty$ & $-\infty$ \\
\hline $\mathrm{s}_{3}$ & $-\infty$ & $-\infty$ & 0 & 0 & 0 & $-\infty$ \\
\hline $\mathrm{s}_{4}$ & 0,8 & $-\infty$ & $-\infty$ & $-\infty$ & 0 & 0 \\
\hline $\mathrm{s}_{5}$ & 0 & 0 & $-\infty$ & $-\infty$ & $-\infty$ & 0,8 \\
\hline $\mathrm{s}_{6}$ & $-\infty$ & $-\infty$ & $-\infty$ & $-\infty$ & 0,6 & 0 \\
\hline $\mathrm{s}_{7}$ & $-\infty$ & $-\infty$ & 0,6 & $-\infty$ & 0,8 & 1 \\
\hline $\mathrm{s}_{8}$ & 1 & 0,8 & $-\infty$ & 0,6 & $-\infty$ & $-\infty$ \\
\hline $\mathrm{s}_{9}$ & 0 & 0 & 0 & 0 & 0 & 0 \\
\hline
\end{tabular}

Q-Matrix - 9. Iteration

\begin{tabular}{|c|c|c|c|c|c|c|}
\hline & $\mathrm{a}_{12}$ & $\mathrm{a}_{13}$ & $\mathrm{a}_{21}$ & $\mathrm{a}_{23}$ & $\mathrm{a}_{31}$ & $\mathrm{a}_{32}$ \\
\hline $\mathrm{s}_{1}$ & 0,51 & 0,64 & $-\infty$ & $-\infty$ & $-\infty$ & $-\infty$ \\
\hline $\mathrm{s}_{2}$ & $-\infty$ & 0,51 & 0,51 & 0,64 & $-\infty$ & $-\infty$ \\
\hline $\mathrm{s}_{3}$ & $-\infty$ & $-\infty$ & 0,64 & 0,51 & 0,51 & $-\infty$ \\
\hline $\mathrm{s}_{4}$ & 0,8 & $-\infty$ & $-\infty$ & $-\infty$ & 0,51 & 0,51 \\
\hline $\mathrm{s}_{5}$ & 0,51 & 0,51 & $-\infty$ & $-\infty$ & $-\infty$ & 0,8 \\
\hline $\mathrm{s}_{6}$ & $-\infty$ & $-\infty$ & $-\infty$ & $-\infty$ & 0,64 & 0,51 \\
\hline $\mathrm{s}_{7}$ & $-\infty$ & $-\infty$ & 0,64 & $-\infty$ & 0,8 & 1 \\
\hline $\mathrm{s}_{8}$ & 1 & 0,8 & $-\infty$ & 0,64 & $-\infty$ & $-\infty$ \\
\hline $\mathrm{s}_{9}$ & 0 & 0 & 0 & 0 & 0 & 0 \\
\hline
\end{tabular}

Figure 9: Q-Matrix after first, second and ninth iteration.

At the beginning of post-optimization, all elements of the Q-Matrix are set to zero. The formula for the recalculation of utility values in Listing 1 is of the same structure as Equation 4. The reward of a state-action-pair $\left(s_{t}, a\right)$ results from the $R R$ matrix. The following state $\mathrm{s}_{\mathrm{t}+1}$ is determined from the $R S$ matrix.

The innermost loop iterates over all actions of a state and the middle loop over all known states. This is a single recalculation of all elements of the Q-Matrix. The outer loop implements the backpropagation approach. The utility values are thereby developed from the target state to the start state, as shown partly in Figure 9. 
The number of repetitions of the computation of all Q-Matrix elements results from the number of known states (row dimension of Q-Matrix). Finally, a scaling of the Q-Matrix values can optionally be done.

All nine states were explored during the ToH example in the training phase. The result of post-optimization is the Q-Matrix after the ninth iteration, as shown in Figure 9. Analogous to the generation of actions on the basis of the learned behavior strategy $\pi$ in Figure 7, the following shorter sequence of state-action-pairs is identified using the post-optimized Q-Matrix:

$\left[\left(\mathrm{s}_{1}, \mathrm{a}_{13}\right),\left(\mathrm{s}_{4}, \mathrm{a}_{12}\right),\left(\mathrm{s}_{7}, \mathrm{a}_{32}\right),\left(\mathrm{s}_{9}\right.\right.$, cancel $\left.)\right]$.

\section{Generating a Robot Control}

With a previously defined behavior strategy $\pi$, an executable control specification can be generated automatically. It is based on the structure of the Simulation Based Control (SBC) approach in [4], as shown in Figure 10.

The control problem is mapped to the control and process model in a task-oriented way. For simple problems such as the ToH example, a separate process model can be dropped.

The interface model (IM) implements a robot type independent task transformation and is implemented with the Robotic Control and Visualization (RCV) Toolbox for MATLAB [3]. An interpreter on the controller of the respective robot acts as the robot type dependent layer according to [3].

The ToH application can be specified using three tasks: (i) move(EulerC), (ii) pick(), and (iii) place(). The task move takes a vector with the six Euler coordinates of the position to proceed to as the parameter EulerC. The other two tasks are for picking and placing a disc. The three tasks are implemented as reusable software components. Due to the simplicity of the tasks, the IM was realized as an integral part of each task.

For the automatic generation of the control specification, a state-action-sequence

$$
\left[\left(\mathrm{s}_{\mathrm{t}}, \exists \mathrm{a} \in \mathrm{A}\right),\left(\mathrm{s}_{\mathrm{t}+1}, \exists \mathrm{a} \in \mathrm{A}\right), \ldots,\left(\mathrm{s}_{\text {target }}, \text { cancel }\right)\right]
$$

must be calculated using the behavior strategy $\pi$. This is done as explained in Section 2 in Figure 7 using the simulated environment. The task-oriented control specification is then derived from the state-action-sequence.

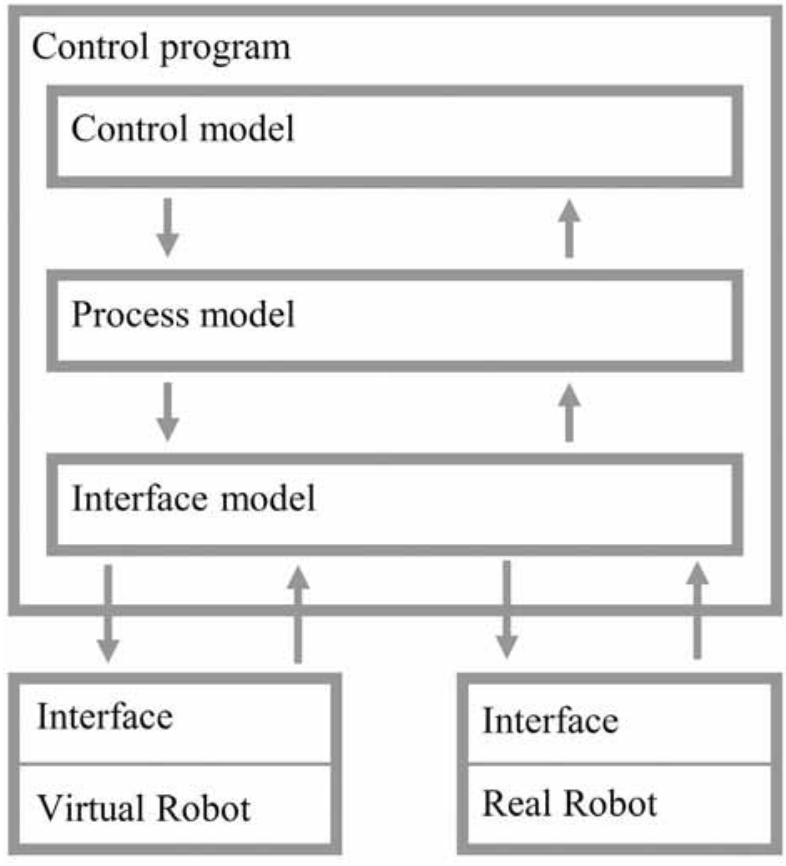

Figure 10: Principle control structure based on the SBC approach.

The ToH application is a typical pick-and-place application. Each state-action-pair $\left(s_{t}, \exists a \epsilon A\right)$ can be executed by the same task sequence:

$$
\text { move }(\text { EulerC }) \rightarrow \operatorname{pick}() \rightarrow \operatorname{move}(\text { EulerC }) \rightarrow \text { place }() \text {. }
$$

The parameter EulerC encodes the x- and y-coordinates of a bar and the z-coordinate for picking or placing the disc. The $\mathrm{x}$ - and $\mathrm{y}$-coordinates of a task result from the action $a$ (Figure 4) and the z-coordinate from the state $s_{t}$ (Figure 2). The angle positions are constant.

In Section 3, the post-optimized state-action-sequence: $\left[\left(s_{1}, a_{2}\right),\left(s_{4}, a_{1}\right),\left(s_{7}, a_{5}\right),\left(s_{9}\right.\right.$, cancel $\left.)\right]$ was calculated for two discs. The four state-action-pairs are transformed into a control, consisting of three task sequences, each of the following form:

$$
\text { move }(\ldots) \rightarrow \text { pick }() \rightarrow \text { move }(\ldots) \rightarrow \text { place }() .
$$

In order to execute the generated control, the coordinates of the bars, the constant angles and the parameters of the disk geometry must be passed to the control program.

The practical implementation took place in the laboratory of the RG CEA with a joint-arm robot of the type KUKA Agilus, as shown in Figure 11. For technical convenience, the discs were replaced by numbered cubes. The learning program has been implemented for any number of discs. On a standard PC, the training phase for four discs took about two seconds. 


\section{Conclusion}

It has been shown, that a task-oriented control for a jointarm robot can be learned offline and simulation-based using an RL method. The control is put into operation by using the SBC approach. Furthermore, it has been shown how the solution of a classic learning process can be improved by post-optimization. For the chosen application example, a typical pick-and-place application, a taskbased control has been successfully learned, generated and executed.

In future work, it will be investigated whether the implemented RL method and the control generation can be applied directly to similar problems or to an entire problem class. Furthermore, applications with human-robot collaboration will be investigated. For those applications, often a complete simulation model of the environment cannot be created and learning has to take place partly online. Hence, it will be examined whether it is possible to learn proactively after the training phase.

In the context of applications with human-robot- or robot-robot-collaboration, RL methods with multiple agents should be investigated.

\section{References}

[1] Weber W. Industrieroboter - Methoden der Steuerung und Regelung (Industrial Robots - Methods of Control and Feedback Control). Carl Hanser Verlag, München, 2008.

[2] ROS.org. wiki.ros.org/Industrial, accessed 03/2018

[3] Deatcu C, Freymann B, Schmidt A, Pawletta T. MATLAB/Simulink Based Rapid Control Prototyping for Multivendor Robot Applications. SNE - Simulation Notes Europe. 2015; 25(2): 69-78. doi: 10.11128/sne.25.2.1029.

[4] Freymann B, Pawletta S, Schmidt A, Pawletta T. Design, Simulation and Optimization of Task-Oriented MultiRobot Applications with MATLAB/Stateflow. SNE Simulation Notes Europe. 2016; 26(2): 83-90. doi: 10.11128/sne.26.2.1033.

[5] Sutton RS, Barton AG. Reinforcement Learning: An Introduction. $2^{\text {nd }}$ Edition. Cambridge/MA: MIT Press; 2012. 334 p.

[6] Akhtar SMF. Practical Reinforcement Learning. $1^{\text {st }}$ Edition. Birmingham/UK: Packt Publishing Ltd.; 2017. $320 \mathrm{p}$.

[7] Watkins CJCH. Learning from Delayed Rewards Ph.D. thesis, Cambridge Univ. / UK; 1989. 241 p.

[8] Kramer O. Computational Intelligence. $1^{\text {st }}$ Edition. Berlin, Heidelberg/DE: Springer Pub.; 2009. 158 p.

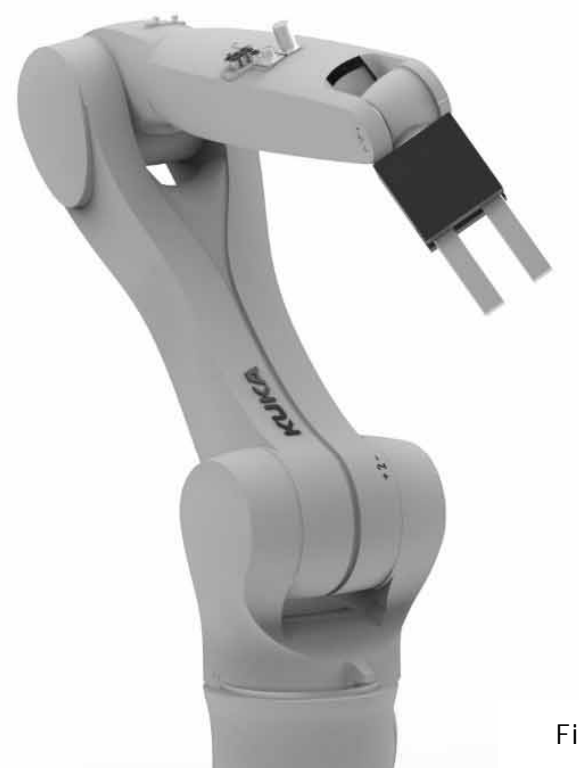

Figure 11: Experimental setup in the laboratory of the RG CEA. 


\title{
Modeling Arterial Wave Reflection with Difference Equations
}

\author{
Laura Lotteraner $^{1 *}, 2$, Bernhard Hametner ${ }^{2}$, Siegfried Wassertheurer ${ }^{2}$ \\ ${ }^{1}$ Institute of Analysis and Scientific Computing, TU Wien, Wiedner Hauptstraße 8-10, \\ 1040 Vienna, Austria; *Iaura.lotteraner@tuwien.ac.at \\ ${ }^{2}$ Center for Health \& Bioresources, AIT Austrian Institute of Technology, Giefinggasse 4, \\ 1210 Vienna, Austria
}

SNE 28(4), 2018, 157-164, DOI: 10.11128/sne.28.tn.10443

Received: September 20, 2018; Revised November 14, 2018;

Accepted: November 20, 2018

SNE - Simulation Notes Europe, ARGESIM Publisher Vienna,

ISSN Print 2305-9974, Online 2306-0271, www.sne-journal.org

Abstract. A simple difference equation model is presented that describes the development of blood pressure in the ascending aorta over time. Reflections both at one and more sites in the arterial system as well as re-reflections of reflected waves at the heart are considered. Model parameters include reflection magnitude, pulse wave velocity, reflection site positions and heart rate. The model is discussed both analytically and numerically by implementation in MATLAB. Comparison of blood pressure curves generated by the model and blood pressure curves measured in patients demonstrates that the model is able to represent reality to some extent. Results can be improved by adding reflection sites which warrants further research.

\section{Introduction}

Hypertension (elevated blood pressure) is a major cause of death mainly but not only in developed countries. According to the World Health Organization [1] hypertension accounts for about $12.8 \%$ of the annual deaths worldwide. In order to improve diagnosis and prevention it is crucial to understand the underlying dynamics in the cardiovascular system.

Mathematical models can help with this. In different types of models, many aspects of blood pressure have been considered by previous researchers. Traditional models are often based on fluid dynamics [2, 3] or use the analogy between the cardiovascular system and an electric circuit [4]. New approaches, however, are possible.
A large number of models involve the description of wave reflections [5], a phenomenon that occurs when blood pressure waves coming from the heart (forward waves) arrive at parts of the arterial system where vessel properties change, such as bifurcations. The forward waves are reflected and produce backward waves. This idea has been further extended by researchers $[6,7]$ who argued that backward waves are re-reflected at the heart. However, only few models exist that address this issue and it is poorly understood so far.

In this paper a simple difference equation model for aortic blood pressure is presented that focuses on the characterization of pulse wave reflections and rereflections. The properties of the model, such as the influence of various parameters on the results, are discussed.

\section{The Model}

Model Design. Even though blood pressure dynamics are time-continuous, time-discrete difference equations are a valuable tool to describe reflection effects from a finite number of reflection sites. Blood pressure at a fixed point in time and space can be calculated as the sum of blood pressure values at previous times, determined by the choice of reflection sites.

The arterial system is considered a single uniform tube with the heart, connected to the system via the aortic valve (AV), on one end and an elastic closed boundary, referred to as (single) distal reflection site (RS), on the other, see Figure 1. This idea has been applied in many different models and has been proved to be a reasonable approach by different authors [8]. It has even been used to model repeated reflections in the arterial system [7]. A measuring site (MS) is fixed in the ascending aorta, close to the aortic valve. This so-called aortic root is of particular interest because it allows to 
assess the interaction of reflections in the vascular system and the heart [9].

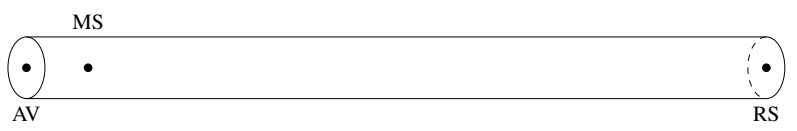

Figure 1: Tube model of the arterial system as presented above. AV - aortic valve, MS - measuring site, RS distal reflection site.

The distance between measuring site and distal reflection site will be named effective length in this paper, a terminology that expresses the reduction of multiple reflection effects to one. Analogously, the distal reflection site is also named effective reflection site. Often $[8,10]$ the aortic bifurcation is mentioned as the principal bifurcation site, the physiological counterpart of the effective reflection site.

Previous tube models have considered at least one spatial dimension and take into account the elastic properties of the tube wall [11]. The model presented in this paper, however, is dimensionless, only depending on time. It describes the development of blood pressure $P$ at the fixed measuring site over time. Therefore all spatial information has to be transformed into temporal information.

Model Parameters. The parameters of the model describe both the heart and the arterial system.

Reflections are described by real-valued reflection coefficients, $0<R_{D}<1$ for the distal reflection site and $0 \leq R^{a v} \leq 1$ for the aortic valve. The reflection coefficients include information on the elasticity properties of the arterial bed and the damping of pulse waves as they propagate through the blood [12]. While $R_{D}$ is assumed to be constant, reflection at the aortic valve can be chosen to be time-dependent [13], i.e. $R^{a v}=\left(R_{k}^{a v}\right)_{k \in \mathbb{N}}$ with the time index $k$ where the length of a time step will be given by the step size $\tau$. Typically, different values are chosen during systole, when the valve is open, and diastole, when the valve is closed.

The number of time steps it takes a pressure impulse to travel from the measuring site to the distal reflection site and back will be denoted by the time constant $t_{b} \in \mathbb{N}$. It includes information on both the distance between measuring site and reflection site and pulse wave velocity. In a more complex description of reflection phenomena the time of return of the reflected wave is also affected by the phase shift that occurs during re- flection [14]. Due to the simplification of the reflection coefficient in this paper this time delay must be included in the time constant. The choice of the time constant is coupled with the choice of the reflection coefficient $R_{D}$ $[14,15,16]$.

Analogously, the number of time steps it takes a pressure impulse to travel from the measuring site to the aortic valve, denoted by $t_{f} \in \mathbb{N}$, is coupled with the choice of $R^{a v}$. Since the measuring site is very close to the valve there holds $t_{f} \ll t_{b}$.

Not only does the heart serve as a reflection site, it also is the driving force for pressure dynamics. During systole blood is pumped into the system, increasing the pressure. This phenomenon is represented by the discrete function $\left(P_{k}^{i n}\right)_{k \in \mathbb{N}}$.

Model Equations and Solutions. In order to formulate the model equations total pressure $P_{k}$ (at the measuring site, at time $k$ ) is split into its forward and backward components $P_{k}^{f}$ and $P_{k}^{b}$, i.e. $P_{k}=P_{k}^{f}+P_{k}^{b}$. Using all the aforementioned parameters the difference equation system

$$
\begin{array}{ll}
P_{k}^{f}=P_{k}^{i n}+P_{k-2 t_{f}}^{b} R_{k-t_{f}}^{a v}, & k \in \mathbb{N}_{>2 t_{f},}, \\
P_{k}^{b}=P_{k-t_{b}}^{f} R_{D}, & k \in \mathbb{N}_{>t_{b}}
\end{array}
$$

can be obtained. Figure 2 illustrates the idea behind equation (1).

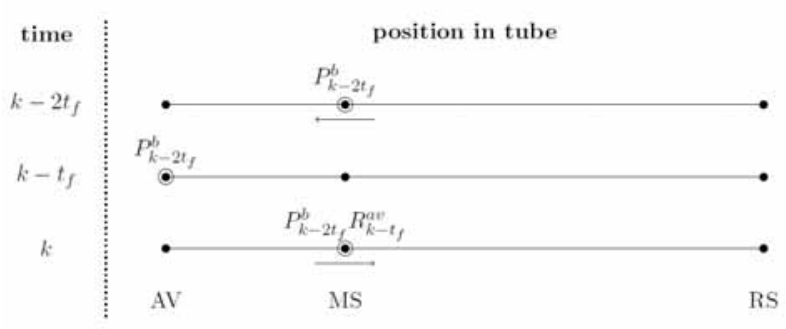

Figure 2: Backward pressure is reflected at the time-dependent aortic valve (AV) as described in equation (1) and returns to measuring site (MS).

Inserting (1) into (2) and vice versa yields the two independent equations

$$
\begin{array}{ll}
P_{k}^{f}=P_{k}^{i n}+P_{k-2 t_{f}-t_{b}}^{f} R_{D} R_{k-t_{f}}^{a v}, & k \in \mathbb{N}_{>2 t_{f}+t_{b},}, \\
P_{k}^{b}=P_{k-t_{b}}^{i n} R_{D}+P_{k-2 t_{f}-t_{b}}^{b} R_{D} R_{k-t_{b}-t_{f}}^{a v}, & k \in \mathbb{N}_{>2 t_{f}+t_{b} .}
\end{array}
$$

Obviously both equations are of order $N$ where $N=$ $2 t_{f}+t_{b}$. Since they only define a recurrence relation 
for $k>N$, initial values $P_{k}^{f}$ and $P_{k}^{b}, k=1, \ldots, N$ are required. Then standard literature [17] guarantees the existence of unique solutions for the resulting initial value problems. The initial values are chosen as $P_{k}^{f}=P_{k}^{i n}$, $k=1, \ldots, N, P_{k}^{b}=0, k=1, \ldots, t_{b}$ and $P_{k}^{b}=P_{k-t_{b}}^{i n} R_{D}$, $k=t_{b}+1, \ldots, N$. Using a solution method for linear difference equations of fixed but unknown order [18] yields the solutions

$P_{k}^{f}=P_{k}^{i n}+\sum_{j=1}^{\left\lfloor\frac{k-1}{2 t_{f}+t_{b}}\right\rfloor} P_{k-j\left(2 t_{f}+t_{b}\right)}^{i n} R_{D}^{j} \prod_{m=1}^{j} R_{k-(m-1)\left(2 t_{f}+t_{b}\right)-t_{f}}^{a v}$

and

$$
\begin{aligned}
P_{k}^{b}=P_{k-2 t_{b}}^{i n} R_{D}+\sum_{j=1}^{\left\lfloor\frac{k-t_{b}-1}{2 t_{f}+t_{b}}\right\rfloor}\left(P_{k-j\left(2 t_{f}+t_{b}\right)-t_{b}}^{i n} R_{D}^{j+1}\right. \\
\\
\left.\prod_{m=1}^{j} R_{k-(m-1)\left(2 t_{f}+t_{b}\right)-t_{f}-t_{b}}^{a v}\right)
\end{aligned}
$$

for $k \in \mathbb{N}_{>2 t_{f}+t_{b}}$.

Model Properties. Using stability theory for linear difference equation systems [17] it can be shown that all the solutions are stable and bounded under the assumption $R^{a v} \equiv 1$. For this purpose both independent equations of order $N$ have to be transformed to an equivalent difference equation system

$$
u_{k+1}=A u_{k}+b_{k+1}
$$

of order one with system matrix

$$
A=\left(\begin{array}{cccccc}
0 & 1 & 0 & \ldots & \ldots & 0 \\
\vdots & 0 & 1 & \ddots & & \vdots \\
\vdots & \vdots & \ddots & \ddots & \ddots & \vdots \\
\vdots & \vdots & & \ddots & \ddots & 0 \\
0 & \vdots & & & \ddots & 1 \\
R_{D} & 0 & \ldots & \ldots & \ldots & 0
\end{array}\right) \quad \in \mathbb{R}^{N \times N}
$$

Then, for any matrix norm $\|\cdot\|$ there holds

$$
\lim _{k \rightarrow \infty}\left\|A^{k}\right\|=0 .
$$

Let $\tilde{A}$ be the system matrix of the equivalent difference equation system of order one with variable $R^{a v}$. Be- cause of

$$
\left\|\tilde{A}^{k}\right\| \leq\left\|A^{k}\right\| .
$$

for any matrix norm $\|\cdot\|$ the same result is valid for this system.

Both functions $\left(R_{k}^{a v}\right)_{k \in \mathbb{N}}$ and $\left(P_{k}^{i n}\right)_{k \in \mathbb{N}}$ are periodic with the same period length $K$ that equals the duration of one cardiac cycle, i.e. the sum of systole and diastole duration. Therefore, also the solutions of the model become periodic with period length $K$ after a certain setting time that depends on the choice of the reflection coefficient $R_{D}$.

Under the assumptions made for the model it is possible to set $t_{f}=0$, i.e. shift the measuring site towards the heart, without major changes in the results. The solution then simplifies to

$P_{k}=P_{k}^{f}+P_{k}^{b}=P_{k}^{i n}+\sum_{j=1}^{\left\lfloor\frac{k-1}{t_{b}}\right\rfloor} P_{k-j t_{b}}^{i n} R_{D}^{j}\left(R_{k}^{a v}+1\right) \prod_{m=1}^{j-1} R_{k-m t_{b}}^{a v}$

for $k \in \mathbb{N}_{>2 t_{f}+t_{b}}$. In order to simplify analysis this will be done in the rest of the paper.

\section{Implementation and Results}

After theoretical discussion the model is implemented in MATLAB. Numerical analysis is conducted by comparing plots of generated curves where one parameter is varied while the others are held constant. Both the influence of parameters on the shape of the curve and on the minimum (diastolic pressure) and maximum (systolic pressure) values are analyzed.

Parameter Values and Implementation. Parameter ranges are determined according to previous studies. Systole duration typically is 0.3 seconds, and diastole duration typically is 0.7 [19]. They are included in the model via the choice of the input function $P^{i n}$ and the reflection at the heart $R^{a v}$.

The distal reflection coefficient should be chosen around $R_{D}=0.5$. Different authors give different values that are computed using different methods $[14,16,20]$.

The same goes for the return time from the distal reflection site where both effective length and pulse wave velocity can be determined in various ways $[14,16,21]$. A typical value is 145 milliseconds which is much shorter than systole duration [22]. This time constant can be transformed to a discrete number of time steps 
using the number of time points per second $1 / \tau$. Implementation of the model using the previously defined parameters and initial values in MATLAB is straightforward.

Input Function and Reflection at the Heart. Two different functions are compared for $P^{i n}$. Both functions are zero during diastole when the valve is closed and the heart produces no output. During systole a half sine that is motivated by other authors' results $[2,13]$ is compared to the much simpler constant one function. The half sine (see Figure 3 ) that considers the opening and closing time of the aortic valve yields more realistic results than the strongly simplified constant function (see Figure4).

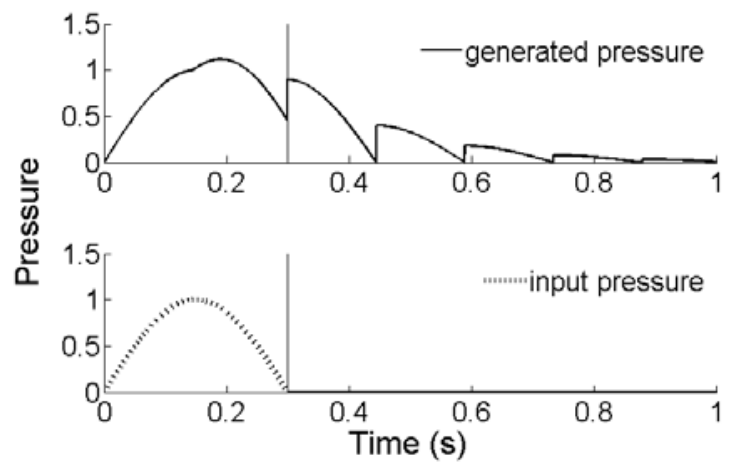

Figure 3: Generated pressure curve (solid) with corresponding input function (dotted). A half sine is chosen during systole. Reflection at the heart is zero during systole and one during diastole. End of systole is indicated by a vertical line. One cardiac cycle.

For modeling reflection at the heart three different functions can be compared. A step function assumes zero during systole when the valve is open and all waves are absorbed and one during diastole when the valve is closed and all waves are reflected. As before, motivation for this function is given by other authors [13]. Instead, the simpler constant functions zero and one can be used. They produce smoother curves with less distinct spikes, see Figure 5. In the following analysis the reflection is assumed to be a step function.

Results. Figure 6 compares a curve generated by the model (solid) with a measured blood pressure curve (dotted) taken from a data set that was collected for a previous study [23]. Both curves are normalized to the

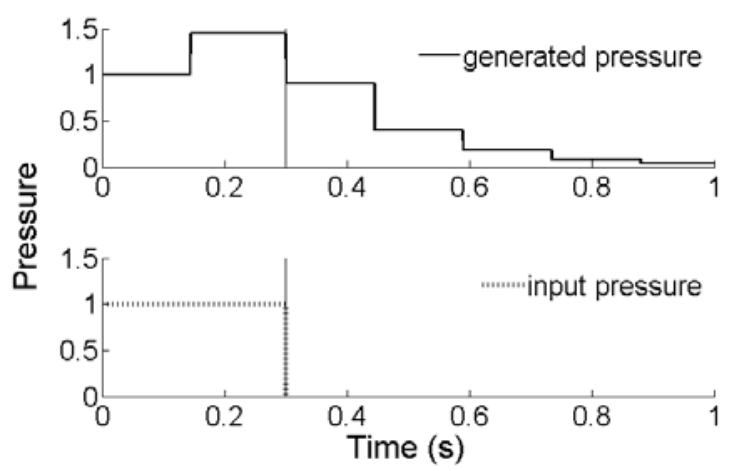

Figure 4: Generated pressure curve (solid) with corresponding input function (dotted). The constant one function is chosen during systole. Reflection at the heart is zero during systole and one during diastole. End of systole is indicated by a vertical line. One cardiac cycle.

same pulse pressure (amplitude) to increase comparability. The focus of this paper is the shape of blood pressure curves, not their absolute values. For the generated curve the input function $P^{\text {in }}$ is chosen as a half sine during systole and zero during diastole. Reflection at the heart, $R^{a v}$, is chosen as the constant one function during systole and zero during diastole. A vertical line indicates the end of systole.

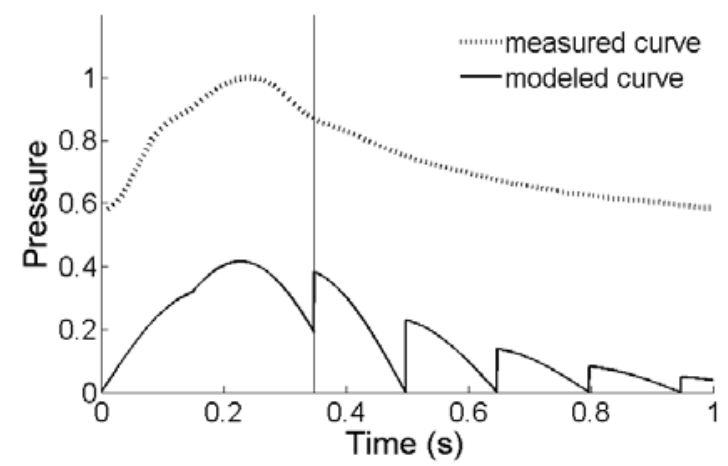

Figure 6: Comparison of a modeled (solid) and a measured (dotted) blood pressure curve. End of systole is indicated by a vertical line. One cardiac cycle.

Even though the modeled curve shows more spikes and is generally less smooth, the main characteristics of an aortic blood pressure curve [24] are represented. There is a (relatively) fast systolic upstroke, a notch at the end of systole and a slow diastolic decay.

Increasing the reflection coefficient $R_{D}$ significantly 


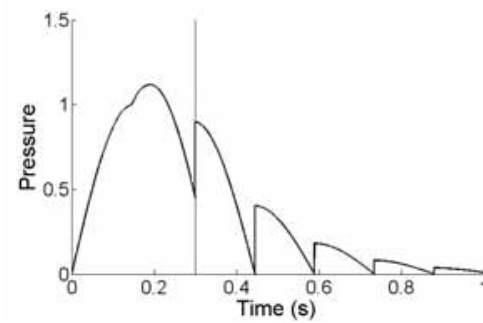

(a) $R^{a v}$ is step function.

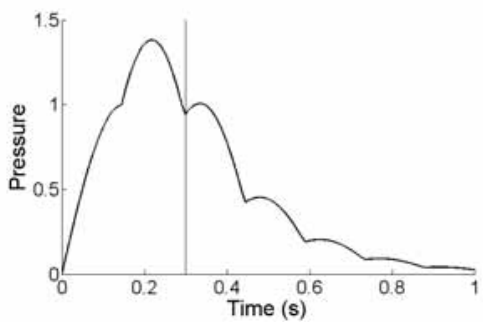

(b) $R^{a v}$ is constant one function.

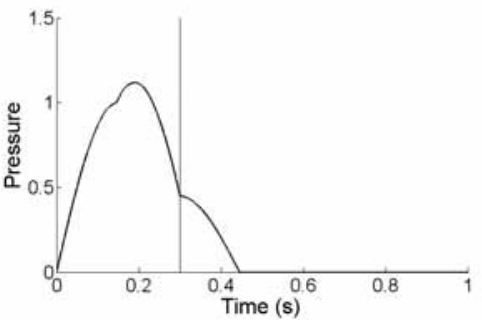

(c) $R^{a v}$ is constant zero function.

Figure 5: Comparison of different shapes of $R^{a v}$. Input function is a half sine during systole and zero during diastole. End of systole is indicated by a vertical line. One cardiac cycle.

increases systolic pressure and the prominence of spikes while diastolic pressure remains close to zero, yielding an increase in pulse pressure, i.e. the amplitude of the wave. The curves look realistic only for a sufficiently small value of $R_{D}$ where the threshold depends on the choice of other parameters.

In assessing the influence of systole and diastole duration one must distinguish between two cases. One can either vary them individually, resulting in a variable heart rate or hold the heart rate fixed which means varying them together. Only the first case that is physiologically more relevant is analyzed in the paper. Systolic pressure increases with systole duration while diastolic pressure remains the same, yielding an increase in pulse pressure, see Figure 7. The prominence of spikes decreases. Both for too low and too high values of systole duration the curve does not resemble a measured one. The thresholds depend on the values of the other parameters. Diastole duration does not have a significant influence on the shape of the modeled curves.

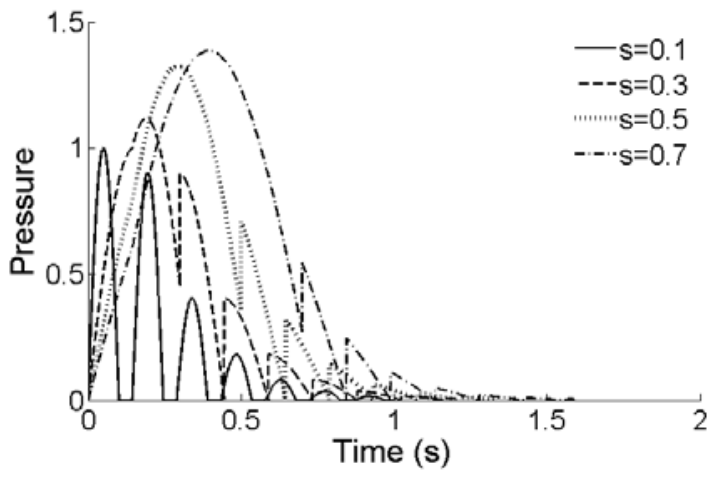

Figure 7: Generated blood pressure curve for four different values of systole duration. One cardiac cycle.

Return time $t_{b}$ negatively influences systolic and pulse pressure while diastolic pressure remains zero. All spikes occurring during diastole become wider and more prominent with increasing return time, see Figures 8 and 9 . The width of spikes during diastole equals the return time, therefore representing the single reflections.

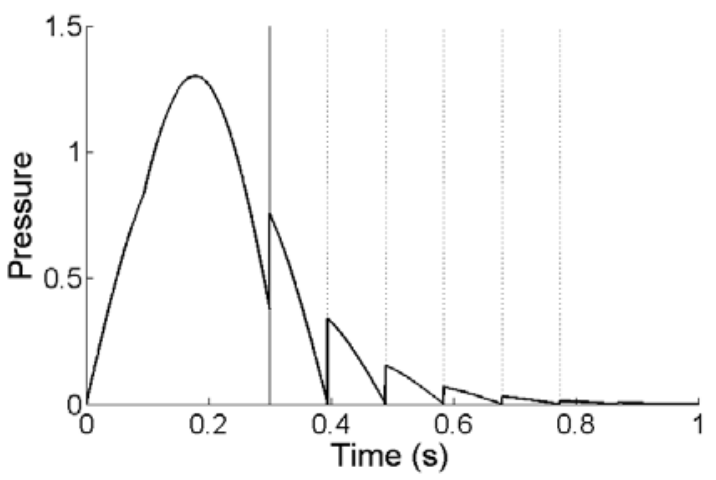

Figure 8: Return time 95ms. End of systole is indicated by a solid vertical line. Return times are indicated by dotted vertical lines. One cardiac cycle.

Choosing a return time that is larger than systole duration does not lead to realistic results.

\section{Model Refinement}

The curves' shapes can be improved by adding more reflection sites. It has been argued before that at least two peripheral reflection sites are necessary to model the entire arterial system - one for the upper and one for the lower body half $[25,26]$.

The Model. While modeling two reflection sites is often accomplished with an asymmetric T-tube model 


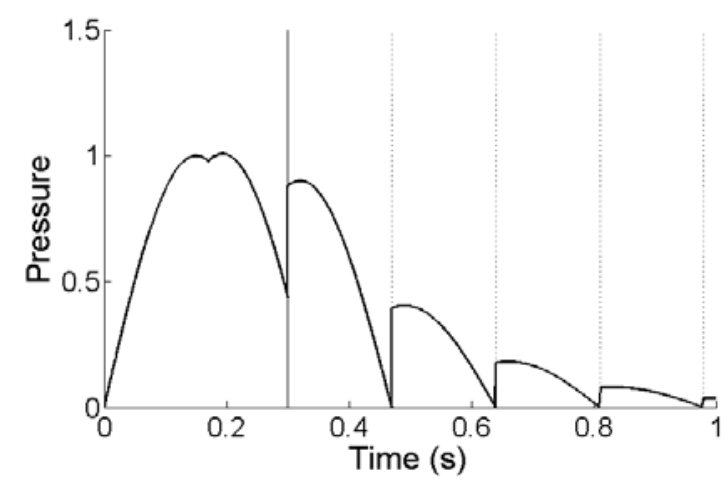

Figure 9: Return time 170ms. End of systole is indicated by a solid vertical line. Return times are indicated by dotted vertical lines. One cardiac cycle.

[27], the reflection sites in the present paper are assumed to be completely independent from each other. The equations are

$$
\begin{array}{ll}
P_{k}^{f}=P_{k}^{i n}+P_{k-2 t_{f}}^{b} R_{k-t_{f}}^{a v}, & k \in \mathbb{N}_{>2 t_{f},}, \\
P_{k}^{b}=P_{k-t_{b}^{1}}^{f} R_{D}^{1}+P_{k-t_{b}^{2}}^{f} R_{D}^{2}, & k \in \mathbb{N}_{>t_{b}^{2}}
\end{array}
$$

where $R_{D}^{1}$ and $R_{D}^{2}$ are the reflection coefficients of the two reflection sites. Their respective return times are $t_{b}^{1}$ and $t_{b}^{2}$ with $t_{b}^{1}<t_{b}^{2}$.

Inserting each of the equations into the other one and prescribing initial values $P_{k}^{f}$ and $P_{k}^{b}$ for $k=1, \ldots, N=$ $2 t_{f}+t_{b}^{2}$ yields two initial value problems. Standard literature [17] guarantees the existence of unique solutions. They cannot be expressed explicitly. It is possible, however, to prove analytically that the solutions of the homogeneous problems are bounded as long as $R_{D}^{1}+R_{D}^{2}<1$. In the following, $t_{f}=0$ will be assumed.

Results. Comparison with the original model reveals that the prominence of spikes during diastole is decreased significantly, see Figure 10. In particular, blood pressure does not decrease to zero after each spike, presumably a result of the superposition of reflected waves. The main characteristics of a blood pressure curve are represented.

It can be observed that the generated curves are both bounded and periodic after a certain setting time, even for $R_{D}^{1}+R_{D}^{2} \geq 1$.

Again, the influence of different parameters can be assessed. While the general effects are the same as in the model with only one reflection site, the different in-

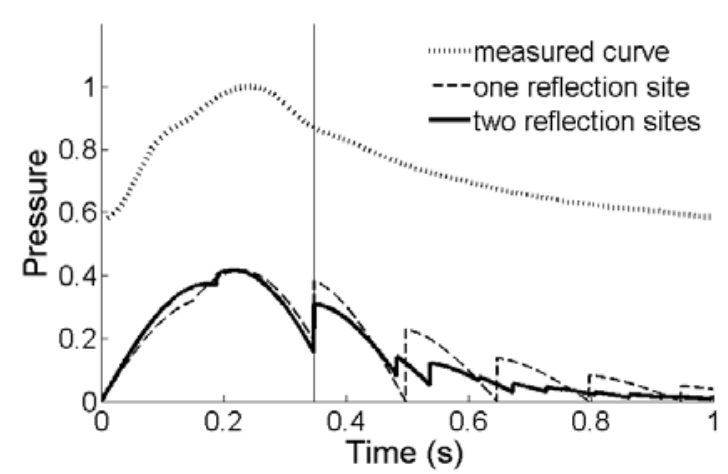

Figure 10: Comparison of a measured curve (dotted), a curve modeled with one reflection site (dashed) and a curve modeled with two reflection sites (solid). End of systole is indicated by a vertical line. One cardiac cycle.

fluences of the two reflection sites can be compared. The reflection coefficients have similar influences on systolic and diastolic pressure as long as both are small. When they exceed a certain threshold that depends on the choice of the other parameters, $R_{D}^{1}$ increases systolic blood pressure and $R_{D}^{2}$ increases diastolic blood pressure more significantly. Physiologically meaningful results can only be obtained when the sum $R_{D}^{1}+R_{D}^{2}$ does not exceed some threshold value that depends on the return times. Each of the reflection coefficients has to be chosen smaller than the reflection coefficient in the original model.

The influence of systole and diastole duration is the same as can be observed in the original model with one reflection site. The same is valid for the influence of both return times.

\section{Conclusion and Outlook}

All the results obtained by theoretical and numerical analysis correspond to effects observed in reality and therefore indicate that modeling aortic blood pressure with difference equations is a reasonable approach.

Instead of a variable reflection at the heart constant functions can be chosen. If the heart is considered to be a total reflector during the entire cardiac cycle, or equivalently, $R^{a v} \equiv 1$, estimating formulas for systolic and diastolic pressure can be found, enabling further theoretical analysis. In fact, choosing $R^{a v}$ close to 1 is necessary 
if physiologically meaningful absolute values, i.e.

diastolic pressure : pulse pressure $\approx 2: 1$,

are desired. This indicates that re-reflections at the aortic valve should not be neglected in methods of arterial pulse wave analysis.

The model can be further refined by adding more distal reflection sites. Also, more complex functions to model reflections at the heart can be chosen. These could be step functions but also piecewise linear functions that consider opening and closing of the valve. Another possibility is to find a model to describes the ventricle. During systole this model is linked with the arterial system while during diastole reflection is assumed to be total.

Once the model has been improved to a desired level of exactness, it can be fit to measured curves by determining the correct parameter combination. From this point, a variety of applications can be considered, such as implementing a wave separation [6] or wave tracking [28] algorithm.

\section{References}

[1] Mendis S, Puska P, Norrving B, editors. Global Atlas on cardiovascular disease prevention and control. World Health Organization, 2011.

[2] Formaggia L, Lamponi D, Quarteroni A. One-dimensional models for blood flow in arteries. Journal of Engineering Mathematics. 2003; 47:251-276. doi: 10.1023/B:ENGI.0000007980.01347.29.

[3] Formaggia L, Perktold K, Quarteroni A. Basic mathematical models and motivations. In: Formaggia, L., Quarteroni, A. and Veneziani, A., editors. Cardiovascular Mathematics. Milano: Springer; 2009. p 47-75.

[4] Westerhof N, Lankhaar JW, Westerhof BE. The arterial Windkessel. Medical and Biological Engineering and Computing. 2009; 47:131-141. doi: 10.1007/s11517-008-0359-2.

[5] Papageorgiou GL, Jones NB. Wave reflection and hydraulic impedance in the healthy arterial system: a controversial subject. Medical and Biological Engineering and Computing. 1988; 26:237-242. doi: 10.1007/BF02447075.
[6] Westerhof N, Sipkema P, van den Bos GC, Elzinga G. Forward and backward waves in the arterial system. Cardiovascular Research. 1972; 6:648-656. doi: 10.1093/cvr/6.6.648.

[7] Berger DS, Li JKJ, Laskey WK, Noordergraaf A. Repeated reflection of waves in the system arterial system. American Journal of Physiology - Heart and Circulatory Physiology. 1993; 264:H269-H281. doi: 10.1152/ajpheart.1993.264.1.H269.

[8] Westerhof N, Stergiopulos N, Noble MIM. Snapshots of Hemodynamics. Boston: Springer; 2005. 192 p.

[9] Burattini R, Knowlen GG, Campbell KB. Two arterial effective reflecting sites may appear as one to the heart. Circulation Research. 1991; 68: 85-99. doi: 10.1161/01.RES.68.1.85.

[10] Caro CG, Pedley TC, Schroter RC, Seed WA. The Mechanics of the Circulation. New York: Cambridge University Press; 2012. 550 p.

[11] Womersley JR. Oscillatory flow in arteries: the constrained elastic tube as a model of arterial flow and pulse transmission. Physics in Medicine and Biology. 1957; 2:178-187. doi: 10.1088/0031-9155/2/2/305.

[12] Nichols WW, O’Rourke MF, Vlachopoulos C. McDonald's Blood Flow in Arteries, Sixth Edition: Theoretical, Experimental and Clinical Principles. London: CRC Press; 2011. 768p.

[13] Wang JJ, Parker KH. Wave propagation in a model of the arterial circulation. Journal of Biomechanics. 2004; 37:457-470. doi: 10.1016/j.jbiomech.2003.09.007.

[14] Westerhof BE, van den Wijngaard JP, Murgo JP, Westerhof N. Location of a reflection site is elusive. Hypertension. 2008; 52:478-483. doi: 10.1161/HYPERTENSIONAHA.108.116525.

[15] Campbell KB, Lee LC, Frasch HF, Noordergraaf A. Pulse reflection site and effective length of the arterial system. American Journal of Physiology - Heart and Circulatory Physiology. 1989; 256:H1684-H1689. doi: 10.1152/ajpheart.1989.256.6.H1684.

[16] Segers P, De Backer J, Devos D, et al. Aortic reflection coefficients and their association with global indexes of wave reflection in healthy controls and patients with Marfan's syndrome. American Journal of Physiology - Heart and Circulatory Physiology. 2006; 290(6):H2385-H2392. doi: 10.1152/ajpheart.01207.2005. 
[17] Agarwal RP. Difference Equations and Inequalities. New York: Marcel Dekker Inc; 1992. 1000 p.

[18] Mallik RK. Solutions of linear difference equations with variable coefficients. Journal of Mathematical Analysis and Applications. 1998; 222:79-91. doi: 10.1006/jmaa.1997.5903.

[19] Thiriet M, Parker KH. Physiology and pathology of the cardiovascular system: a physical perspective. In: Formaggia, L., Quarteroni, A. and Veneziani, A., editors. Cardiovascular Mathematics. Milano: Springer; 2009. p 1-45.

[20] Latham RD, Westerhof N, Giolma JP, Altobelli SA. Regional wave travel and reflections along the human aorta: a study with six simultaneous micromanometric pressures. Circulation. 1985; 72:1257-1269. doi: 10.1161/01.CIR.72.6.1257.

[21] Murgo JP, Westerhof N, Giolma JP, et al. Aortic input impedance in normal man: Relationship to pressure wave forms. Circulation. 1980; 62: 105-116. doi: 10.1161/01.CIR.62.1.105

[22] Sherwin SJ, Franke V, Peiro J, Parker KH. One-dimensional modelling of a vascular network in space-time variables. Journal of Engineering Mathematics. 2003; 47:217-250. doi: 10.1023/B:ENGI.0000007979.32871.e2.
[23] Weber T, Auer J, O’Rourke MF, et al. Arterial Stiffness, wave reflections, and the risk of coronary artery disease. Circulation. 2004; 109:184-189. doi: 10.1161/01.CIR.0000105767.94169.E3.

[24] Stoner L, Young JM, Fryer S. Assessments of arterial stiffness and endothelial function using pulse wave analysis. International Journal of Vascular Medicine. 2012; Article ID 903107.. doi: 10.1155/2012/903107.

[25] O'Rourke MF, Taylor MG. Input Impedance of the System Circulation. Circulation Research. 1967; 20:365-380. doi: 10.1161/01.RES.20.4.365.

[26] Sipkema P, Westerhof N, Randall OS. The arterial system characterised in the time domain. Cardiovascular Research. 1980; 14:270-279. doi: 10.1093/cvr/14.5.270.

[27] O'Rourke MF. The arterial pulse in health and disease. American Heart Journal. 1971; 82:687-702. doi:10.1016/0002-8703(71)90340-1.

[28] Parker KH. The reservoir-wave model. Artery Research. 2017; 18:87-101. doi:10.1016/j.artres.2017.04.003. 


\title{
Autonomous Landing System: Safe Landing Zone Identification
}

\author{
Aljaž Blažič ${ }^{1}$, Klemen Kotnik ${ }^{1}$, Kristina Nikolovska ${ }^{1}$, Miha Ožbot ${ }^{1}$, Martin Pernuš ${ }^{1}$, \\ Uroš Petkovič ${ }^{1}$, Nika Hrušovar ${ }^{2}$, Matic Verbič̌, Irena Ograjenšek ${ }^{2}$, Andrej Zdešar ${ }^{1}$, \\ Matevž Bošnak ${ }^{1}$, Tine Tomažič ${ }^{3}$, Gregor Klančar ${ }^{1 *}$ \\ ${ }^{1}$ Faculty of Electrical Engineering, University of Ljubljana, Trzaška 25, 1000 Ljubljana, Slovenia; \\ *gregor.klancar@fe.uni-lj.si \\ 2 Faculty of Economics, University of Ljubljana, Kardeljeva ploščad 17, 1000 Ljubljana Slovenia \\ 3 PIPISTREL VERTICAL SOLUTIONS d.o.o, Vipavska cesta 2, 5270 Ajdovščina, Slovenia
}

SNE 28(4), 2018, 165 - 170, DOI: 10.11128/sne.28.tn.10444

Received: November 12, 2018; Revised November 21, 2018;

Accepted: December 3, 2018

SNE - Simulation Notes Europe, ARGESIM Publisher Vienna

ISSN Print 2305-9974, Online 2306-0271, www.sne-journal.org

\begin{abstract}
Recently, there has been a growing interest in developing autonomous landing systems which is the greatest challenge of the whole autonomous flight mission. This paper presents market research results of currently available sensors for environment recognition during landing. Main focus is given to development of algorithms for the safe-landing zone estimation using LIDAR and stereo-camera sensors. And finally, the paper highlights challenges in this research field.
\end{abstract}

\section{Introduction}

A new revolutionary innovation in transportation is approaching which will allow traveling by air and will reduce both costs and time spent while increasing comfort. Similarly in the past, a bit more than a century ago first cars appeared that enabled the development of mass mobility. Nicolas-Joseph Cugnot built the first selfpropelled road vehicle, a military tractor running on steam, while the first gasoline powered car was invented by Karl Friedrich Benz in 1885. Recently five successful companies started partnership with Uber to elevate an electric flying taxi to service in 2020 [3]. One of them is also Slovenian company PIPISTREL VERTICAL SOLUTIONS d.o.o. [4], who also joined the student project PKP (entitled "By creative way towards knowledge"), and whose activity is summarized in this article.
The project is co-financed by the European Union from the European Social Fund and the Republic of Slovenia. Seven students and five mentors from the Faculties of electrical engineering and Economics in Ljubljana and the company PIPISTREL VERTICAL SOLUTIONS d.o.o. have cooperated in this project. The main purpose of this project is that students acquire additional competencies and knowledge that can help them with career and employment. During the project the students need to solve specific problems, and actual challenges defined by the participating company.

One of the key challenges of such autonomous VTOL (vertical takeoff and landing) aircrafts is the development of an automated and safe landing system. Before landing, the system needs to identify an appropriate landing surface where the aircraft can safely land. The identification of a safe landing zone cannot depend only on the pilot's view from the cabin as he may have incomplete information. Therefore we propose an automated sensor system that would assist the pilot, and in the future it will also allow autonomous operation. The proposed system is based on 3D LIDAR and stereo camera measurements, which are processed in the algorithms to obtain depth images of the environment. These sensors turned out to be currently the most appropriate selection for the task.

We decided to use and upgrade existing solutions due to the economies of scale, where the price of the final product decreases with demand on the market. That would also allow to offer the product to global market in the future. 
In the following, the overview of the existing sensors currently available on the market is given, followed by the description of the developed algorithms for sensor data processing, identification of landing zone from the sensor measurement and appropriate presentation of the results to the user. Realistic measurements were taken to test and validate the proposed ideas. Finally, some future challenges in the area of research are exposed.

\section{Landing Problem and Used Development Environments}

The main goal of the project was to propose a conceptual idea how to solve autonomous landing of VTOL aircraft. The proposal includes the selection of appropriate sensors and analysis of their possible placements to the aircraft, the required sensor measurements processing and development of the basic algorithms that would illustrate the main idea. First, the main assumption, starting points and limitations were defined as follows:

- $\quad$ the system needs to be operational for heights up to 50 meters,

- final decision for the landing must be given at least at a height of $15 \mathrm{~m}$,

- $\quad$ used sensors should not include mechanically spinning parts to increase robustness, sensors need to be fixed or only rotation in one direction is permitted,

- more sensors can be arranged over the hull of the aircraft,

- the whole cost of the sensor system should not exceed $10.000 €$

During the development, the following tools were used:

- MATLAB, where the main development of the algorithms was performed. Due to many libraries, easy work with matrices, easy debugging and visualization, it allows rapid development of algorithm ideas and processing of data. This environment was used to implement algorithms and for data processing from LIDAR and imaging camera.

- PCL (Point cloud library), which is an open source library for 2D and 3D image and data clouds processing. It contains algorithms for filtering, surface reconstruction (modelling and segmentation), object of known shape recognition etc. The library contains several sub libraries. It supports various sensors, such as stereo cameras, 3D scanners, Kinect and similar. PLC library was used in the project for processing images from stereo camera.

- Fusion 360, which is a 3D modelling or drawing program. Its advantage is that it contains all phases of product development (CAD, CAM in CAE) in a single platform. It is a free access program for students. We used it for drawing 3D models and also for the analysis of various scenarios of sensor selection and their positioning on the aircraft model.

\section{Sensor Overview for Autonomous Landing Purpose}

Sensors are the most important elements in autonomous landing systems. When selecting appropriate sensors, the preferred are those who have at least $30 \mathrm{~m}$ range, have no rotating mechanical parts and are sufficiently robust.

The main goal of the performed analysis is an overview of the available sensors in the market, identifying which of those are still in the development phase and will be available soon and their types. We have checked the entire offer (2D, 3D and 1D LIDAR, cameras, stereo cameras, IR cameras, radars, ToF cameras, ultrasound sensors, etc.) and selected the most appropriate ones for our needs (see Table 1).

We have found out that many suppliers offer solutions, which are still under development and as such unavailable and usually with uncomplete specifications. An example of such disappointment were Solid-State LIDAR sensors, which would be the optimal choice as they have no moving parts (e.g. rotating mirrors) and are therefore more robust. However, the majority of them is currently unavailable (still in development) or have a small field of view, short range, rough resolution and a high price.

On the contrary, a very high potential have imaging sensors (camera, stereo camera), which are already available and their measurement (image) includes a high amount of information. However, a bigger challenge when using cameras is the quality of image processing, in order to extract the desired information from the measurements, such as reliable 3D images or point clouds of the environment from larger distances. 
IR cameras operation depends on temperature contrast of the environment which can in a given area fluctuate a lot. Nevertheless, the camera returns a 2D image, suitable for processing and rapid analysis of potential objects. ToF cameras only operate at very short distances and in good conditions (clear weather). Their good side is that they can directly measure 3D information. Radars can operate on both short and long distances, but are limited by poor resolution.

The choice of currently the most suitable sensors for our solution, which are available on the market, is shown in Table 1.

Four different scenarios for landing area detection were suggested. They all share the same basic idea in acquiring a $3 \mathrm{D}$ cloud of space points, which is then processed to identify good and bad areas for landing.

\begin{tabular}{ll}
\hline Sensor type & Sensor \\
\hline Solid-State LIDER & Cepton HR80W \\
\hline Stereo camera & MultiSense S2 \\
\hline Infrared camera & NightHawk 2 \\
\hline Time of Flight & 03D303 \\
\hline Radar & Continental ARS4-A \\
\hline
\end{tabular}

Table 1: Selected sensors.

In the classification of the planes, the RANSAC (RANdom SAmple Consenses) method turned out as the most appropriate. Each scenario contains a combination of two different sensor principles where the weaknesses of the one is eliminated by the advantages of the other. The following combinations of sensors are made:

- solid-state LIDAR combined with stereo camera,

- IR camera combined with stereo camera,

- Radar combined with stereo camera and

- ToF camera combined with stereo camera.

All mentioned approaches are based on gradual measurements, where the use of the sensor at different heights improves the accuracy and reliability of the taken measurements. Integration of several sensors in a solution also improves redundancy, thereby increasing safety in the event of a malfunction. More information sources are obtained for fusion and comparison to other sources of information.
Some sensors, e.g. solid state LIDAR, have a very narrow range of measurement $(\mathrm{FoV})$ such as, for example, $60^{\circ} \times 24^{\circ}$ in the Cepton HR80W (Wide Angle) model. A possible solution (less robust) here is a mechanical mechanism that would move the sensor in a circular arc and thus capture several individual images, which are merged into a common image of the terrain. Another solution (more expensive and more demanding) is the installation of several identical sensors, which at the same time cover a larger area.

\section{Safe Landing Zone Estimation}

\subsection{Basic approach}

The block diagram of the data processing from the capture of the measurements to the graphic representation is given in Figure 2. It is constructed in a way that it is common to both the LIDAR measurements and the stereo camera measurement obtained by the constructed depth image.

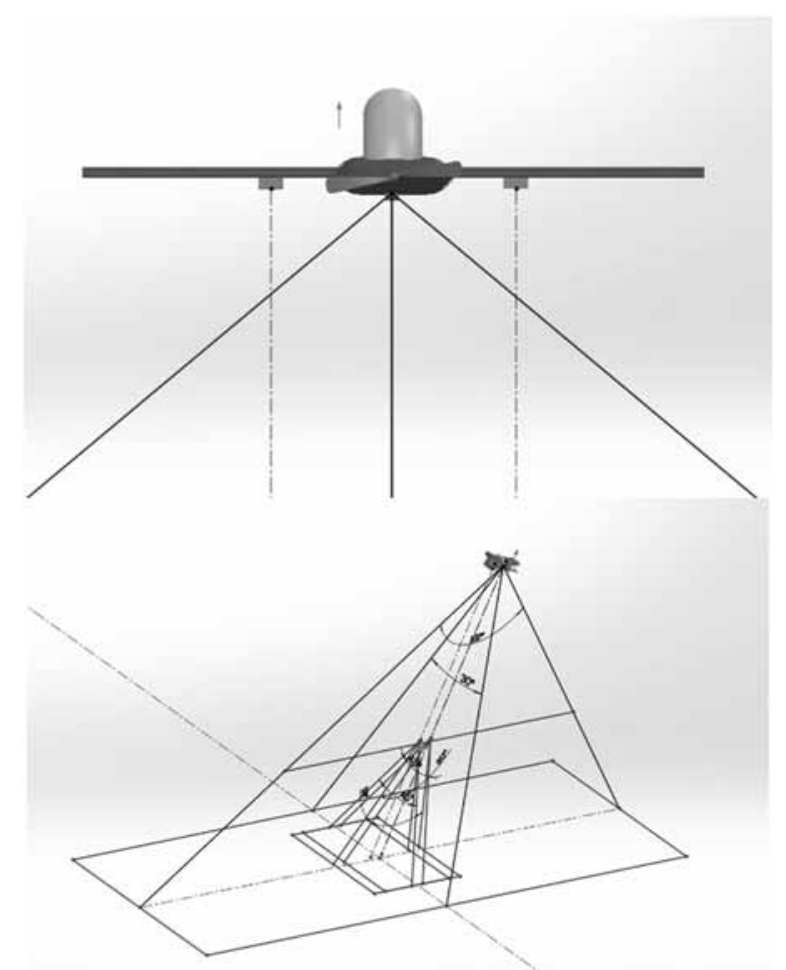

Figure 1: Sensor placement on the aircraft (LIDAR sensor on the middle and stereo cameras on the wings) and image from the analysis of obtained field of views. 


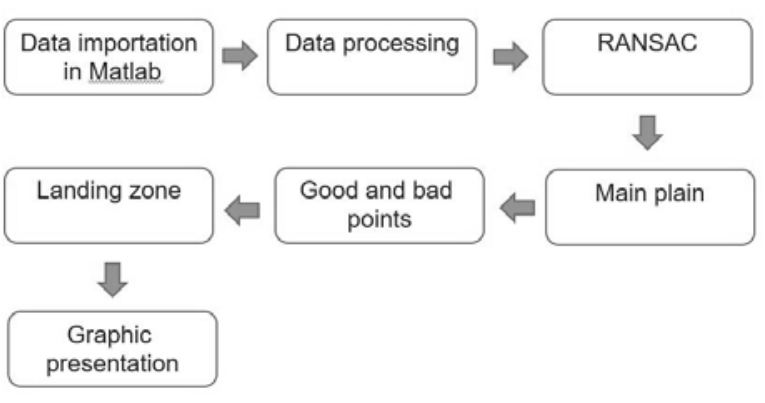

Figure 2: Work flow of data processing from captured measurements towards graphic presentation.

\subsection{Performing measurements}

Development and operation of algorithms were carried out exclusively on real measurements, which were captured from two different sensors, i.e. LIDAR (Velodyne VLP-16) and stereo camera (two ACME VR06 at a distance of $1 \mathrm{~m}$ ). Measurements were taken from a window on the second floor of the Faculty of Electrical Engineering (view of the parking lot, Figure 3).

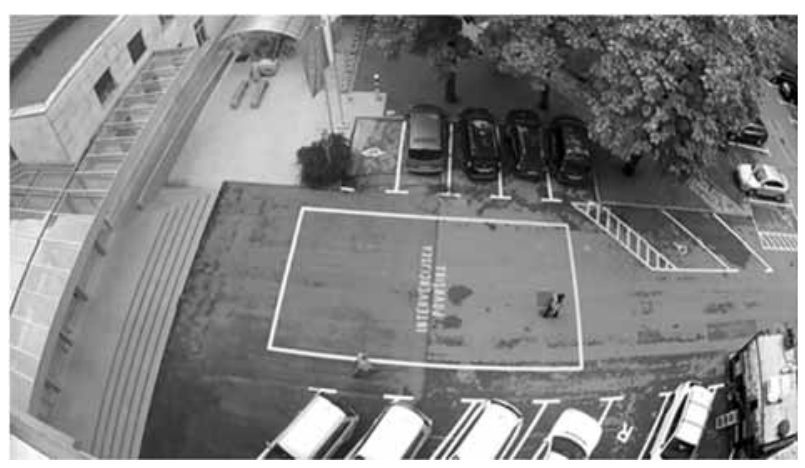

Figure 3: View of the area that was measured.

\subsection{Approach using LIDAR sensor}

Data from Velodyne VLP-16 LIDAR was obtained in the form of a pcap (Velodyne packet capture) file, which can be transformed into a csv (comma-separated values) format using VeloView.

We imported the data into MATLAB using the csvread function, where the result is a cloud of points given in the Cartesian coordinate system of the LIDAR sensor (Figure 4). The Velodyne VLP-16 measures $360^{\circ}$ field of view, but only a specific area of this measurement is interesting for our problem therefore irrelevant points were removed.

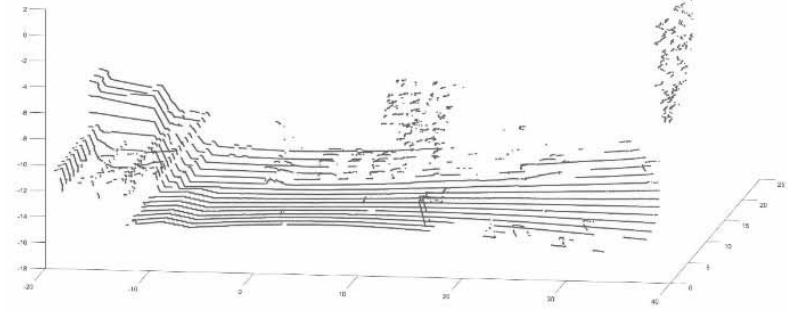

Figure 4: 3D depth image obtained from the LIDAR.

The brief idea of the implemented safe landing area algorithm is as follows. From the obtained measurement determine the boundaries of the area and divide it into sub-areas. Using the RANSAC method in each subregion, find a plain that fits best to the points in that region. RANSAC is an iterative process for estimation of the parameters of a mathematical model from data. Data can contain outliers which must not influence the estimation of parameters. The algorithm first checks if there are enough points, otherwise the sub-area is marked as an undefined area. If there are enough points, three points are randomly selected, which accurately determine a plane. In the next step, the distances of all points of the sub-area to the plane are calculated. All the points that are in a certain proximity (e.g. at least 10 $\mathrm{cm}$ ) of the plane are recorded and considered as good points. The described procedure is repeated n-times. We only save the list of points when their number is higher than in all previous experiments.

Good points can be classified also if they have been marked more than m-times as close enough to a randomly found plane.

After completion of the iterations, the largest list of good points is stored and the plane, which best fits the good points, is determined by the least-square method. The resulting plane is evaluated based on how close it is to the horizontal plane. It can also be validated by the alignment of the plane's normal vector with z-axis (of the scene) and by the total distance of all the good points from it. On the basis of deviations (from the normal direction and points distances), the obtained plane is estimated to be acceptable or unacceptable, or as a good or a bad zone.

In the next step, the main plane (in Figure 5-6, indicated by dark green) is determined taking into account all good planes of the sub-areas. This can be done in several different ways. Good plains at different heights must not be joined. 
Follows the classification of all points based on the distance from the main plane (with threshold e.g. 10 $\mathrm{cm}$ ) to determine if points are good or bad. Additionally the surroundings of all bad points are checked where the first good point is searched around a given bad, which is subsequently interpreted as a bad point. This creates information on the edges of the obstacles. All the given points are mapped into the main plane, where new "undefined" points are added in the areas where the points are too far away from each other. New undefined points do not contain any other points in the radius $\mathrm{R}$.

Then the largest radius containing good points is searched to obtain a candidate for the best landing surface. Additionally, the whole searched area needs to be within the boundaries of the main plane and must not be in contact with an undefined area. The search of the best landing area is an iterative process where the search area moves along $x$ and $y$ axes and saves the most successful area found.

The final result of the mentioned recognition process is the graphic presentation (Figure 5, first method) of the main plane with the illuminated circle (light green) representing the suitable landing zone, and a slightly darker green color on the plane shows good points and the red color shows the obstacles.

In the second presentation approach (Figure 6) the results are calculated first in each sub-region, by calculating the average value by the median. In the undetermined regions, this average value is determined from the median of the neighborhood average values. Then all these obtained points are connect to form a continuous surface.

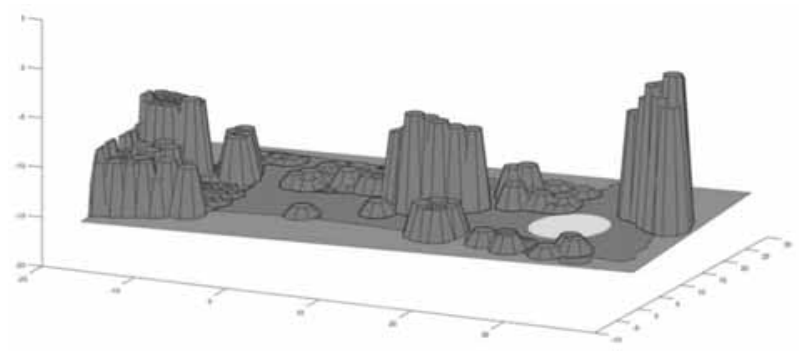

Figure 5: Presentation of the results obtained by the first method.

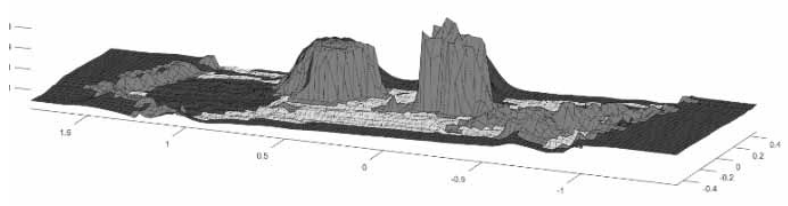

Figure 6: Presentation of the results obtained by the first method.

\subsection{Stereo camera approach}

Using the stereo camera which consists of two ACME VR06 cameras (placed $1 \mathrm{~m}$ apart), measurements of the same environment (Figure 3) as in the case of LIDAR were performed. After applying appropriate calibration of the stereo camera, a corresponding depth image is obtained. It turned out that a quality of calibration is of great importance in obtaining a proper depth image. In the following, the process of obtaining depth images is described.

The first step in image processing is the calibration of the stereo camera. The calibration provides parameters describing the camera's internal parameters and external parameters. The internal parameters represent the optical center and focal length of the camera. While the external parameters represent the orientation and distance transformation between the two cameras. Due to the use of cameras with a wide angle, it is important to use radial coefficients of distortion, which take into account the grater deviations of the light rays at the edges of the lens as compared to the optical center. As usually, the calibration was performed using a chessboard image that is in the field of view of both cameras. In each image, the vertices of the chessboard are searched. By using several image frames with marked vertices and information on the true distance between individual vertices, the necessary internal and external parameters as well as the distortion parameters can be calculated following the algorithm described in article [1].

By using calibration parameters, the distortion in the images can be corrected and compensated. Further on by matching the images, it is achieved that the corresponding points in both images are in the same row, or the same values of the y coordinate of the image.

In order to achieve this, a transformation of the image is done, which makes the same result as it would be obtained if the cameras were parallel and at the same height. By obtaining transformed images, a picture of the disparity between the two images is calculated. The disparity represents the difference in the pixels between the columns of the correspondence points on the left and the right image.

Calculation of the disparity was carried out using semi-global matching method [2]. This method differs from the usual methods for disparity calculation because it requires a similar disparity between the nearby blocks (groups) of pixel elements. 
This can partially eliminate noise, which appears due to the lack of image elements in the search for a disparity.

The cloud of points from the found pairs of image pixels is obtained by the following transformation:

$$
\left[\begin{array}{cccc}
1 & 0 & 0 & -c_{x} \\
0 & 1 & 0 & -c_{y} \\
0 & 0 & 0 & f \\
0 & 0 & -1 / T_{x} & \left(c_{x}-c_{x}^{\prime}\right) T
\end{array}\right]\left[\begin{array}{l}
x \\
y \\
d \\
1
\end{array}\right]=\left[\begin{array}{c}
X \\
Y \\
Z \\
W
\end{array}\right]
$$

where $c_{x}$ and $c_{x y}$ are the center of the left camera sensor, $f$ is the focal length of the left camera, $T$ is the distance between the cameras, $c_{x}^{\prime}$ is the $x$-coordinate of the center of the right camera and $d$ is the dispersity. Since we deal with homogeneous coordinates, the pixel position in $3 \mathrm{D}$ coordinates is equal to $(X / W, Y / W, Z / W)$.

When the point cloud is obtained, it is processed in the same manner as with the LIDAR data (Figure 2 and Subsection 3.3). The result of the processing is shown in Figure 7.

Each point of the depth image in Figure 7 represents $10 \times 10$ pixels in the original image. If the point is good it is colored green or if it is bad it is colored red. In the case where the point in Figure 7 is not specified, the corresponding image elements are shown in black.

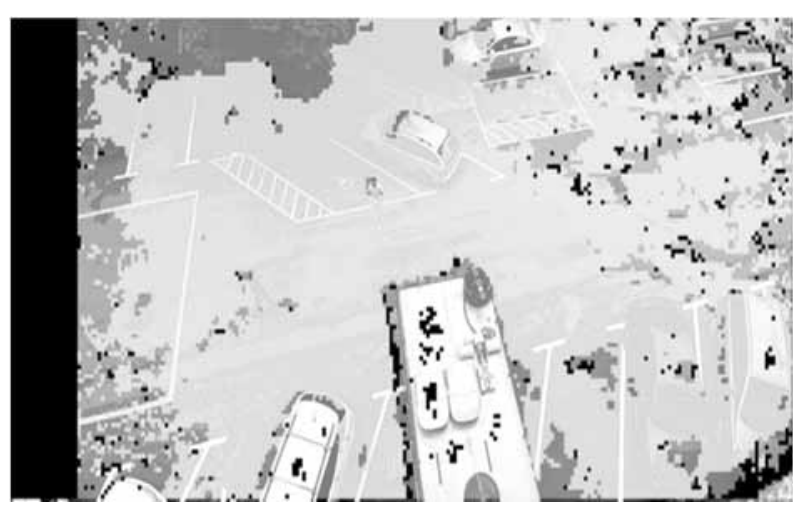

Figure 7: Depth image obtained from the stereo camera. View of good (green) zones, bad (red) zones and undefined (black) zones on the left camera image.

\section{Conclusion}

The paper presents a proposal for the design of an autonomous landing system and an automatic estimation of the safe zone based on 3D LIDAR and stereo cameras. Based on the performed overview of sensors it is concluded that many sensors that are suitable (e.g. solidstate LIDAR) are still in the development phase.
This study highlights mainly the limitations and advantages of the available sensors currently on the market.

The results obtained in the evaluation of the proposed algorithms operation are good, considering the expected problems in machine vision even above expectations. Algorithms obtained in this phase of research cannot run in real time, as the primary goal was to validate proposed concepts, algorithms, and a set of sensors. The obtained results of the research suggest that it is possible to obtain a reliable autonomous zone detection system using available and affordable sensors and existing algorithms.

Further improvements and steps for future development would be the following. Validate the proposed methodology also on higher quality sensors, especially cameras. Upgrade of the algorithms to obtain greater robustness, by choosing more reliable features in determining image disparities and optimization to achieve real-time operation. Acquisition of real measurements with the proposed sensors on real aircraft and performing possible improvements in the sensors positioning, their selection and in recognition algorithms.

\section{Acknowledgement}

The presented work was performed in the frame of PKP project, which is co-financed by the European Union from the European Social Fund and the Republic of Slovenia.

\section{References}

[1] Zhengyou T. A flexible new technique for camera calibration. IEEE Transactions on pattern analysis and machine intelligence. 2000; vol. 22: pp.1330-1334.

[2] Hirschmuller H. Accurate and Efficient Stereo Processing by Semi-Global Matching and Mutual Information. International Conference on Computer Vision and Pattern Recognition. 2005; vol. 2: pp. 807-814.

[3] Uber Elevate, Fast-Forwarding to a Future of OnDemand Urban Air Transportation, available at: https://www.uber.com/elevate.pdf, 2016.

[4] Pipistrel d.o.o., Uber partners with Pipistrel Aircraft to manufacture electric VTOLs, available at: https://www.pipistrel.si/news/uber-partners-withpipistrel-aircraft-to-manufacture-electri, 2017 


\title{
Different Strategies for Modelling and Simulation of the Impact of Migration on Regional Population Development
}

\author{
Matthias Obermair $^{1 *}$, Martin Bicher ${ }^{1,2}$, Felix Breitenecker ${ }^{1}$ \\ ${ }^{1}$ Institute of Analysis and Scientific Computing, TU Wien, Wiedner Hauptstraße 8-10, \\ 1040 Vienna, Austria; *matthiasobermair@gmail.com \\ ${ }^{2}$ dwh simulation services, dwh GmbH, Neustiftgasse 57-59, 1070 Vienna, Austria
}

SNE 28(4), 2018, 171-176, DOI: 10.11128/sne.28.tn.10446 Received: November 5, 2018; Revised November 30, 2018; Accepted: December 2, 2018

SNE - Simulation Notes Europe, ARGESIM Publisher Vienna, ISSN Print 2305-9974, Online 2306-0271, www.sne-journal.org

\begin{abstract}
Demographic developments are not only a point of interest on national but also on sub-national level where migration rates are typically much higher compared to international movements. This article presents several models for the simulation of the impact national and international migration has on regional population development. Hereby we contribute to the research field of demographic modelling as we demonstrate pros and cons of the different modelling strategies when trying to parametrize the models with real-world data. Since the influence of an individual's age and sex on its mobility is a common feature discussed in literature, the models developed mainly focus on these influences. While the first models use decoupled migration processes, one additional approach is designed to take into account an individual's wish of moving to a particular place and not being distributed to a random region. All models are further enhanced with a region-specific external migration tool.
\end{abstract}

\section{Introduction}

Demography plays a very important role in many aspects of governmental and economic planning. Many essential features of our society, such as the health-care system, need reliable data on population development, in this special case e.g. to guarantee an unfailing provision of medical care.

Tom Wilson[1] formulated three answers to the question, why in particular internal migration is worth a detailed examination. Firstly, for understanding the dynamics of a country's population geography, it is necessary to understand internal migration patterns. Secondly, to compute population estimates in years where no census takes place, reliable (internal) migration data are essential. Wilson thus argued that "internal migration is often the most important demographic variable shaping regional population age structures." Thirdly, multi-regional demographic prospects by single ageclasses of course need precisely modelled migration profiles by single age-classes.

To embrace the influence of (internal) migration on regional population developments, this article further investigates its impact on a country's demography and how to model it in the most fitting way with respect to the data available for the regions in question. Therefore different model approaches will be presented and tested and their results compared. The models were implemented using the object-oriented programming language Python 3, altering a given basic model which will be presented in the next section. Among other advantages like free accessibility and an enormous pool of freely available Python packages, this language is capable of dealing with the high number of required agents.

\section{The Generic Population Concept (GEPOC)}

In 2015 a generic population model, capable of producing a valid virtual image of Austria's population and feasible prognoses, only using public accessible initial population data, was completed as part of the Decision Support for Health Policy and Planning (DEXHELPP) health-care research project. 
Amongst other things, the GEPOC model was implemented as an agent-based model which was further developed as part of this work. It is defined by its initial setup and time-dynamics.

- Initial setup: Once a simulation start date is fixed, an agent-based model with $N+1$ agents is initialized. $N$ of them represent the inhabitants of Austria. Each of these agents can be imagined as representative of a real person and receives an individual birth-date and sex (male or female) as well as a unique ID. The empirical data used for the initial population is provided by the Austrian Bureau of Statistics[2]. The additional agent shall be referred to as government-agent, as it will play the role of the government.

- Time-dynamics: The model is updated in apriori defined time-steps which are not necessarily equidistant. For every time-step all agents representing individuals are iterated in random order. For each one the model decides if they die, emigrate or (in case of a female agent) give birth to a new agent, using an event-based strategy. In case of death or emigration the agent is removed from the model and possibly later scheduled events are skipped. The birth event leads to the construction of a new agent with a birth-date according to the schedule. After the iteration is completed, the government-agent generates a given number of immigrants and adds them to the model as new agents.

After the systematic generation of results, they have been validated using prognosis data of the Austrian $\mathrm{Bu}-$ reau of Statistics[2]

\section{Mathematical Models for Internal Migration}

Henceforth (if not stated otherwise) migrant, immigrant and emigrant always refers to internal migration.

Various reasons and factors have to be considered when doing research on internal migration. For example Aude Bernard, Martin Bell and Elin CharlesEdwards concentrate on the life-course transitions that affect migration choices - especially of young adults directly, calling them "proximate determinants". They state that economic, social and other rather general factors, shape people's plans for their lives and therefore lead to migration age profiles. Age profile differences between countries occur through different timing of entry into education, labour market entry, partnership and childbearing[3]. The approach presented by them will be further explained in Section 2.1. Wilson and Bell state that multi-regional models with fixed migration rates tend towards dampening the net migration gains in fast growing regions and therefore causing convergent regional growth rates. The reason for this is that in such a fast growing region the potential number of emigrants is increasing at the same speed as the population, whereas the pool of possible immigrants cannot keep that pace. In addition, model approaches, where migration flows are influenced by both origin and destination, deliver better representations of migration behaviour compared to those depending merely on the original population size in the base year[4].

\subsection{Migration age profile model (MAP)}

Various scientists who have examined internal migration age profiles in different countries, have often found a high degree of regularity over space and time for migration age profiles, although huge variations in levels of migration occur[1]. Thus, they suggest the usage of generalised, age-dependent migration probabilities. The big advantage of such a MAP model is the small amount of additional input data for internal migration. It only consists of one matrix per gender, where migration flows between all regions (including the region of origin) for the required time are listed. A typical standardised migration age profile is presented by Aude Bernard, Martin Bell and Elin Charles-Edwards [3]. Migration propensities per age undergo a high variation, with young adults forming the most mobile group. Mobility peaks between twenty and thirty and declines steadily afterwards, with an eventual rise around retirement age and again shortly before death. Many authors consider this strong empirical regularity as almost universal, which is shown by the wide use of Tom Wilson's "age schedule of migration" [5].

A disadvantage of generalised migration age profiles is, according to David Plane, that fixed origindestination migration rates cannot be justified on behavioural grounds, since true dynamics of regional demographic change are not reflected by them [6]. Figure 1 shows the existing regional differences in agedependent migration intensities for Austria. Since this work concentrates especially on internal migration and the differences between the regions concerning a mi- 
gration age profile, this approach has been rejected and replaced by improved models with higher accuracy.

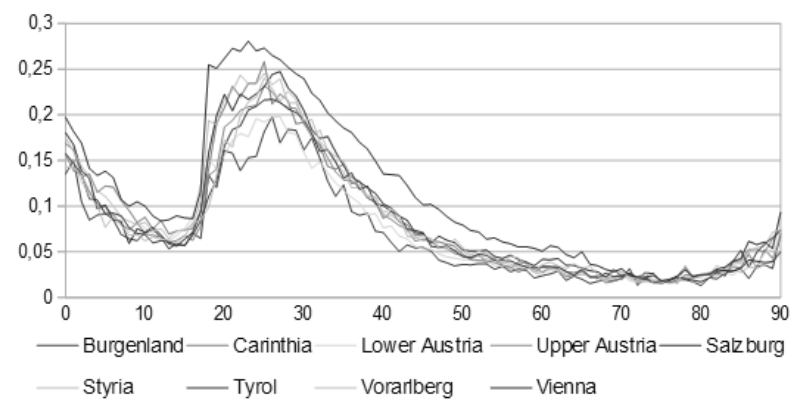

Figure 1: Differences in age-dependent migration probabilities between the federal states of Austria in 2017 according to Statistik Austria for ages 0-90[2]. Although a common tendency is visible, regional differences cannot be overseen. Especially Vienna shows higher internal migration rates due to its high population density.

\subsection{Biregional model (BR)}

With the MAP model handling only migration probabilities and not the whole migration process between regions, the first well-defined modelling approach for internal migration is the construction of a so-called biregional model, based on the one presented by Wilson and Bell[4]. The additional data needed for the internal migration flows consists of initial population as well as emigration and immigration data for every region partitioned into sex (s) and age (a). In fact, this approach is the combination of several biregional models instead of one whole multi-regional model. It is assumed that the country in question is divided into one region of interest and a combination of all other regions. Then emigration and immigration rates are calculated for this particular region. This process is repeated for every region in the simulation.

$$
\begin{aligned}
\operatorname{emr}_{i, s, a} & =\frac{\operatorname{EM}_{i, s, a}(T)}{P_{i, s, a}(T)} \\
\operatorname{imr}_{j, s, a} & =\frac{\operatorname{IM}_{j, s, a}(T)}{\sum_{i} P_{i, s, a}(T)}
\end{aligned}
$$

The emigration rate corresponds to the total emigration per sex and age of the region divided by the total population of the region per sex and age at start time $T$ of the simulation. The immigration rate (2) is calculated as the total number of immigrations per sex and age to a particular region from all other regions combined, divided by the total population per sex and age of the country at start time $T$.

For the implementation of the biregional model since the GEPOC model is agent-based - it has to be evaluated for every agent if they emigrate. With that knowledge the calculation of the total amount of emigrants, which naturally has to be the same as the total amount of immigrants for internal migration, is possible. To ensure that the overall net internal migration sums to zero, it is necessary to rescale the calculated immigration rate. Now all emigrating agents can be distributed to their destination. This process is repeated for every time-step, leading to a very costly procedure. Therefore in the second approach the internal simulation was implemented via a migration pool model.

\subsection{Migration pool model (MP)}

Another model based on the paper of Wilson and Bell[4] is the so-called migration pool model. In theory it is executed in two steps: first the number of all emigrants from all regions is evaluated and the migrants concerned are put into a common "pool." Second they are divided into different regional destinations. While the emigration rate is calculated analogous to Equation (1) in the BR model, the difference to the BR model is that this division is not based on the population but on the total number of migrants.

To obtain the total number of immigrations to each region, immigration rates have to be calculated in the form of immigrants of one region separated by sex and age divided by total immigrants of the corresponding group

$$
\operatorname{imr}_{j, s, a}=\frac{\operatorname{IM}_{j, s, a}(T)}{\sum_{j} \operatorname{IM}_{j, s, a}(T)} .
$$

Equation (4) depicting the migration flow between two regions, shows the major advantage the MP model has compared to the BR model in an agent-based approach.

$$
\mathrm{M}_{i, j, s, a}(t)=\mathrm{emr}_{i, s, a} \cdot \mathrm{P}_{i, s, a}(t) \cdot \mathrm{imr}_{j, s, a}
$$

Since the migration flow is calculated as the product of two fixed rates and the group-specific population of one region, rescaling is not necessary. This results in an agent's probability $\mathrm{m}$ to migrate from region $i$ to region j

$$
\mathrm{m}_{i, j, s, a}=\mathrm{emr}_{i, s, a} \cdot \mathrm{imr}_{j, s, a} .
$$


Under these assumptions it is not necessary to actually create a pool of emigrants in an intermediate step. Therefore during the simulation the whole migration process of one individual can be handled in one quick step. Still, in the MP model immigration into regions is only dependent on the pool size and not the composition of the pool by region and origin [7].

\subsection{Inter-regional migration model (IRM)}

All models presented above have in common that emigration and immigration happen independently, thus there is no connection between origin and goal region. The fact that an individual emigrates from region $A$ or $B$ has no influence on their decision where to immigrate to afterwards. Although this has no effect on the number of migrants and the total population, from an agentbased point of view it should be considered. Since individuality is the unique feature of $\mathrm{ABM}$, the agents in an internal migration model should decide where to immigrate based on their initial region. The inter-regional migration model reaches this goal by changing the internal migration process. While the decision to emigrate is still dependent on the emigration rate analogous to the BR and MP model, for immigration decisions age is not incorporated. Instead data which represents movement from every initial region to all goal regions is included. Thereby, while the agent's age is still important since it influences its emigration decision, the agent's destination is dependent on its origin.

Formalising this leads to an inter-regional migration rate for each sex from region $i$ to region $j$ which can be seen in Equation (6):

$$
\operatorname{imr}_{i, j, s}=\frac{\operatorname{IM}_{i, j, s}(T)}{\sum_{j} \operatorname{IM}_{i, j, s}(T)}
$$

Note that now the distribution and number of immigrants to one region depend on their origin instead of their age. The new migration rate between two regions thus is:

$$
\mathrm{m}_{i, j, s, a}=\mathrm{emr}_{i, s, a} \cdot \mathrm{imr}_{i, j, s}
$$

\subsection{Enhancement with region-specific external migration (rem)}

Until now all models have concentrated on internal migration only, thus external migration is handled for the conglomerate of all regions within the model. Emigration to a foreign country happens on the basis of a common probability for all agents, whereas immigrants from abroad are split proportionally according to the population of the regions.

With the additional region-specific external migration approach, every region has specifically adapted immigration and emigration rates for abroad. To implement this, foreign migration data for every region used has to be added, implying additional parametrisation data. Still, it has the big advantage of improving the simulation results significantly, which will be shown later on.

\section{Results}

The MP model has the highest accuracy of the standard models. The BR and MP models produce very similar results, with the MP approach being slightly more accurate, whereas the IRM model differs from them. The simulation results for the latter show the by far biggest deviation compared to the data. While for the first two approaches the relative difference overall constitutes a maximum deviation of $4.41 \%$ for the BR and 3.64\% for MP model respectively (both Vienna, 2016), the difference for the IRM model reaches a deviation of up to $7.23 \%$ (Vienna, 2017). For all three approaches the growth of Vienna drags behind in favour of the other, less densely populated areas close to Vienna. With the MP model having a quicker computation time, it is satisfying to observe that it produces the best results of the basic implementations.

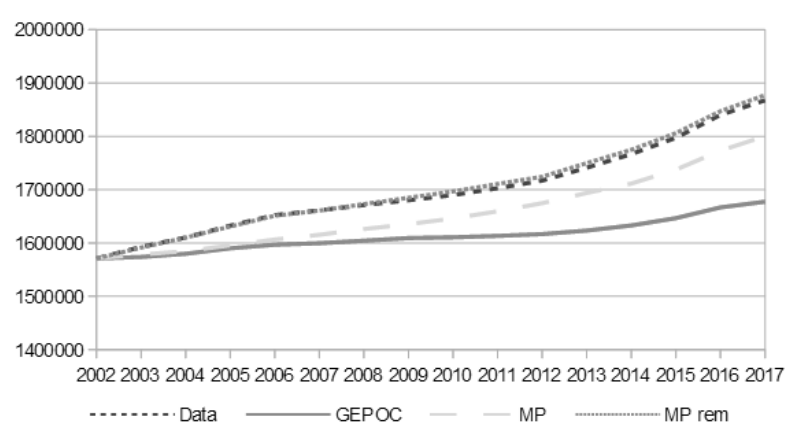

Figure 2: Comparison of the population of Vienna according to data, original GEPOC (without internal migration), the MP model and the MPrem.

Enhancement with region-specific external migration pays off. For all three upgrades a considerable improvement of simulation results can be ob- 
served. Figure 2 exemplary shows this improvement for the MP model. While for Vienna the difference between data and outcome differ up to 81.952 people in 2017 for the MP approach, the upgraded MPrem reaches a maximum discrepancy of (minus) 9.802 for Vienna. Still, compared to the actual population of Vienna in 2017 of 1.867 .582 inhabitants, the results of both approaches are rather accurate. The relative difference of the MP model upgraded with region-specific external migration now diminishes to a maximum of 1.99\% (Upper Austria, 2017). The enhancement enables the successful transition of the (too many) people living in regions like Lower Austria, according to the standard MP model, to Vienna. The distribution of external immigrants changes from the allocations solely depended on population density in the standard models, to a distribution according to the real data. The magnitude of this change is the same for all approaches and for external emigration.

The IRMrem model has the highest accuracy of all models implemented. Of all modelling approaches compared, the IRMrem model reaches the most accurate results for the years 2002-2017. The highest deviation reaches an absolute number of 22.437 persons, compared to 29.173 in the second-best performing MPrem approach (both Upper Austria, 2017). Since the basic IRM model has the worst accuracy, the highly increased performance of the IRMrem model is very interesting. Apparently the internal immigration decision which is directly dependent on the origin of the agent adds up well with the region-specific external migration. This observation will be further investigated in the next paragraph. Considering the very good runtime performance of the IRMrem approach, this model is the best option to choose if the necessary data for the region-specific external migration is available.

\section{Overall simulated migration rates are too low.}

A closer look at the internal migration data shows that - with very few exceptions - the number of migrants calculated tends to be lower than the actual ones. The rates are too low, especially for Vienna. This finding is in line with the results concerning the population figures. Again the BR and MP models produce similar results with the IRM model dragging behind. Altogether, the implementation of region-specific external migration has a positive impact on internal emigration rates, too. The similar behaviour patterns of the approaches derive from the use of the same data and the identical data processing for the internal emigration process. The difference between the IRM model and the other two develops over time and is a consequence of the significantly lower internal immigrations happening right from the beginning.

The simulation forecasts produce very similar results. The population forecasts until 2030 produced by the different modelling approaches are nearly akin. Comparing them, the MP model predicts more people living in the city of Vienna, with accordingly less in the countryside. The IRM model mirrors the contrary development and the BR model is somewhere in between those two. These trends are confirmed when the internal migration rates of all simulation results are compared. Thus, the observations of the timespan 2002-2017 persist for the forecast, although, through the comparably simple extrapolation of the migration data by linear regression, the informative value of the predicted population development is questionable.

All models presented require demographic data available from most national (European) institutes for statistics or Eurostat, respectively[8]. The type and amount of parametrisation data required is listed in Table 1 .

\section{Conclusion}

The main part of this article concentrated on the expansion of the existing demographic GEPOC model with internal migration to simulate regional population changes. All implemented models are constructed to handle an arbitrary amount of regions, as long as data for the migration flows between them is provided. The analysis and validation was undertaken for the federal states of Austria. In conclusion the inter-regional migration model with region-specific external migration turned out to be the best performing one, regarding both computation time and accuracy of the results.

Internal migration has a high impact on simulating regional population numbers accurately. As it has been shown in this work, regional demographic development cannot be simulated accordingly without regionspecific external migration. Still the necessity of additional data for this enhancement has to be considered. The IRMrem approach needs fewest parametrisation data of all models with region-specific external 


\begin{tabular}{llr}
\hline Model & Parameter & Datapoints \\
\hline GEPOC & Emigration & 3.584 \\
& Immigration & 3.584 \\
& Birth & 1.792 \\
& Death & 3.584 \\
& TOTAL & 12.544 \\
\hline BR & GEPOC & 12.544 \\
$\&$ & Internal Emigration & 32.256 \\
MP & Internal Immigration & 32.256 \\
& TOTAL & 77.056 \\
\hline IRM & GEPOC & 12.544 \\
& Internal Emigration & 32.256 \\
& Internal Immigration & 2.592 \\
& TOTAL & 47.392 \\
\hline BRrem & Emigration & 32.256 \\
$\&$ & Immigration & 32.256 \\
MPrem & Birth & 1.792 \\
& Death & 3.584 \\
& Internal Emigration & 32.256 \\
& Internal Immigration & 32.256 \\
& TOTAL & 134.400 \\
\hline IRMrem & Emigration & 32.256 \\
& Immigration & 32.256 \\
& Birth & 1.792 \\
& Death & 3.584 \\
& Internal Emigration & 32.256 \\
& Internal Immigration & 2.592 \\
& TOTAL & 104.736 \\
\hline
\end{tabular}

Table 1: Parametrisation data necessary for the implemented models using a timespan of 16 years, 112 different age groups and nine regions.

migration. The generation of accurate population development forecasts is very difficult due to the unavailability of data concerning future migration trends.

The developed agent-based models can be further enhanced and used for many different applications. A possible usage lies in the analysis of probable developments of local labour markets. Various other possibilities lie in planning regional infrastructure like schools, retirement homes, hospitals and public transport.

\section{References}

[1] Wilson T. The impact of education-bound mobility on inter-regional migration age profiles in Australia. Applied Spatial Analysis and Policy. 2015; 8(4):371-391.

[2] Statistik Austria. STATcube. http: //statcube at. Accessed: 2018-07-18.

[3] Bernard A, Bell M, Charles-Edwards E. Life-Course Transitions and the Age Profile of Internal Migration. Population and Development Review. 2014; 40(2):213-239.

[4] Wilson T, Bell M. Comparative empirical evaluations of internal migration models in subnational population projections. Journal of Population Research. 2004; 21(2):127.

[5] de Jong PA, Brouwer AE, McCann P. Moving up and down the urban hierarchy: age-articulated interregional migration flows in the Netherlands. The Annals of Regional Science. 2016;57(1):145-164.

[6] Plane DA. Requiem for the Fixed-Transition-Probability Migrant. Geographical Analysis. 1993;25(3):211-223.

[7] Van der Gaag N, Van Imhoff E, Van Wissen L. Internal migration scenarios and regional population projections for the European Union. International Journal of Population Geography. 2000;6(1):1-19.

[8] Commission E. Eurostat. https: //ec.europa.eu/eurostat/data/database. Accessed: 2018-09-18.

[9] Bicher M, Glock B, Miksch F, Schneckenreither G, Popper N. Definition, Validation and Comparison of Two Population Models for Austria. In: Book of Abstracts, 4th International Conference on Business, Technology and Innovation. 2015; .

[10] Ravenstein EG. The laws of migration. Journal of the statistical society of London. 1885;48(2):167-235. 


\title{
Decision Trees for Human Activity Recognition Modelling in Smart House Environments
}

\author{
Veralia Gabriela Sánchez ${ }^{*}$, Nils-Olav Skeie \\ Department of Electrical Engineering, Information Technology and Cybernetics, \\ University of South-Eastern Norway (USN), Porsgrunn, Norway; *veralia.g.sanchez@usn.no
}

SNE 28(4), 2018, 177-184, DOI: 10.11128/sne.28.tn.10447 Received: November 27, 2018; Revised December 6, 2018; Accepted: December 10, 2018

SNE - Simulation Notes Europe, ARGESIM Publisher Vienna, ISSN Print 2305-9974, Online 2306-0271, www.sne-journal.org

Abstract. Human activity recognition in smart house environments is the task of automatic recognition of physical activities of a person to build a safe environment for older adults or any person in their daily life. The aim of this work is to develop a model that can recognize abnormal activities for assisting people living alone in a smart house environment. The idea is based on the assumption that people tend to follow a specific pattern of activities in their daily life. An open source database is used to train the decision trees classifier algorithm. Training and testing of the algorithm is performed using MATLAB. The results show an accuracy rate of $88.02 \%$ in the activity detection task.

\section{Introduction}

Human activity recognition modelling (HAM) in smart environments is an important area of research. Smart houses are being developed to improve and ease the life of the inhabitant. The idea of implementing HAM is to recognize the activities of a person in order to adapt the house to its user $[1,2]$.

A smart house is defined as any living environment that has been carefully designed to support its inhabitant in carrying out daily activities, as well as to promote independent lifestyles [3].

People tend to follow a pattern in their daily live $[4,5]$. Therefore, it is possible to recognize the activities of daily life (ADL) a user performs, such as eating, toileting, bathing, dressing, etc. This recognition task is also known as human activity recognition (HAR).

Once the ADL recognition task is done, HAM can use the output from it to learn the pattern of the user and model the user's activities. The modelling has the potential to detect any deviation from the usual pattern.

Detecting abnormal activities has several applications including assisting older adults. In Norway, $38.5 \%$ of households with people aged 65 and over are living alone [6]. Hence, a smart house can help the older adult to remain living in their own home for as long as possible [7].

In this work, HAR is implemented using an open source database. The output of the HAR is used for the HAM. HAM generally refers to the task of modelling the person activity pattern together with time. Therefore, accurate activity recognition is a crucial part for good HAM.

Decision trees are used to develop the HAM. Decision trees are a probabilistic algorithm that is able to predict the next step or value by learning from data. An open dataset is used for training the model.

\section{Related Work}

Decision tree is a supervised learning method. This method has been used for several tasks in the field of pattern recognition and machine learning as a predictive model. The main goal is to predict the next value given several input variable.

Previous studies on pervasive environment using decision trees have been successfully implemented [8].

In smart house environments, an $80 \%$ accuracy was achieved using decision trees on 20 everyday activities in a research by Bao and Intille ([9]). Another research based on decision tree with good result for ADL is the work by Fan et al. ([10]).

\section{Design and Methods}

Figure 1 shows the methodology flow in this work. 


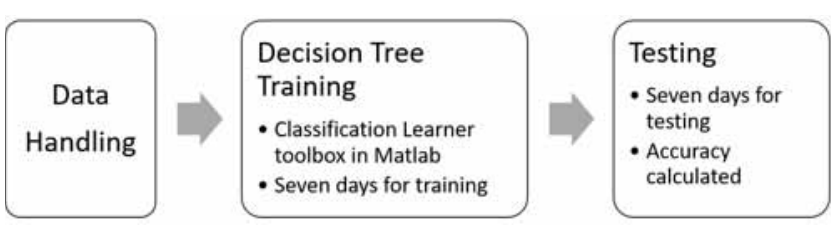

Figure 1: Methods.

\subsection{Decision trees}

Decision trees is a hierarchical model also known as classification and regression trees. They have the property of predicting response from data.

The attributes of the decision trees are mapped into nodes. The edges of the tree represent the possible output values. Each branch of the tree represents a classification rule, from the root to the leaf node [11].

\subsection{Software}

MATLAB is used in this study for developing the model. The classification learner toolbox was specifically used for training the tree. The code from the classification learner toolbox was exported and saved for later use with any other dataset. Testing was also coded in MATLAB.

Finally, the program Wolfram Mathematica is used for the results plots presented in this article.

\begin{tabular}{|c|c|}
\hline Name & Value \\
\hline Setting & Apartment \\
\hline Number of Rooms & 4 Rooms + Hall/Entrance \\
\hline Number of labelled days & 14 days \\
\hline Labels (ADLs included) & $\begin{array}{l}\text { Leaving, Toileting, Show- } \\
\text { ering, Sleeping, Breakfast, } \\
\text { Lunch, Dinner, Snack, } \\
\text { Spare Time/TV, Grooming }\end{array}$ \\
\hline Number of sensors & 12 sensors \\
\hline Sensors & $\begin{array}{l}\text { PIR: Shower, Basin, Cook- } \\
\text { top } \\
\text { Magnetic: Maindoor, } \\
\text { Fridge, Cabinet, Cupboard } \\
\text { Flush: Toilet } \\
\text { Pressure: Seat, Bed } \\
\text { Electric: Microwave, } \\
\text { Toaster }\end{array}$ \\
\hline
\end{tabular}

Table 1: ADLs Database.

\subsection{Dataset}

An open dataset is used in this study. The dataset has been previously used in other research and is known in the HAR field [12]. The dataset is named "Activities of Daily Living (ADLs) Recognition Using Binary Sensors Data Set" and is available for download at [13]. The purpose of using an open dataset is to obtain unbiased results.

The dataset consists of annotated ADLs collected by two different users living on a daily basis in a smart house. The activities in the dataset were manually labelled by the users. Table 1 presents the dataset attributes.

Two instances of data exist corresponding to each user living in the smart house. One dataset of 14 days (OrdonezA), and the second dataset of 21 days (OrdonezB). The first dataset data is depicted in Figure 2. The first dataset is used this work for creating and testing the model. The second dataset (OrdonezB) is implemented later in order to test the model with a different dataset.

Data Handling The variables used from the dataset are "Date", "Time", "Activity", and "Room". Another variable named "position" was added to improve the recognition task. This variable position correspond to one of the three following values: laying, sitting, standing.

Table 2 depicts the first day from the dataset. The dataset is in a text file format.

In order to model the decision tree, a sample was drawn from the dataset. All the 14 days in the dataset were stopped when the activity leaving was found. Seven days were used for training and seven days were used for testing.

The dataset text values were coded to numbers in order to develop the MATLAB code. Table 3 shows the rooms with their respective codes.

Numbers were also assigned to the activities to make the learning and decoding process more feasible.

Table 4 shows the activities with the assigned codes.

Table 5 shows the coding used for the position values.

A total of 9 activities, 5 rooms, and 3 positions are used. 

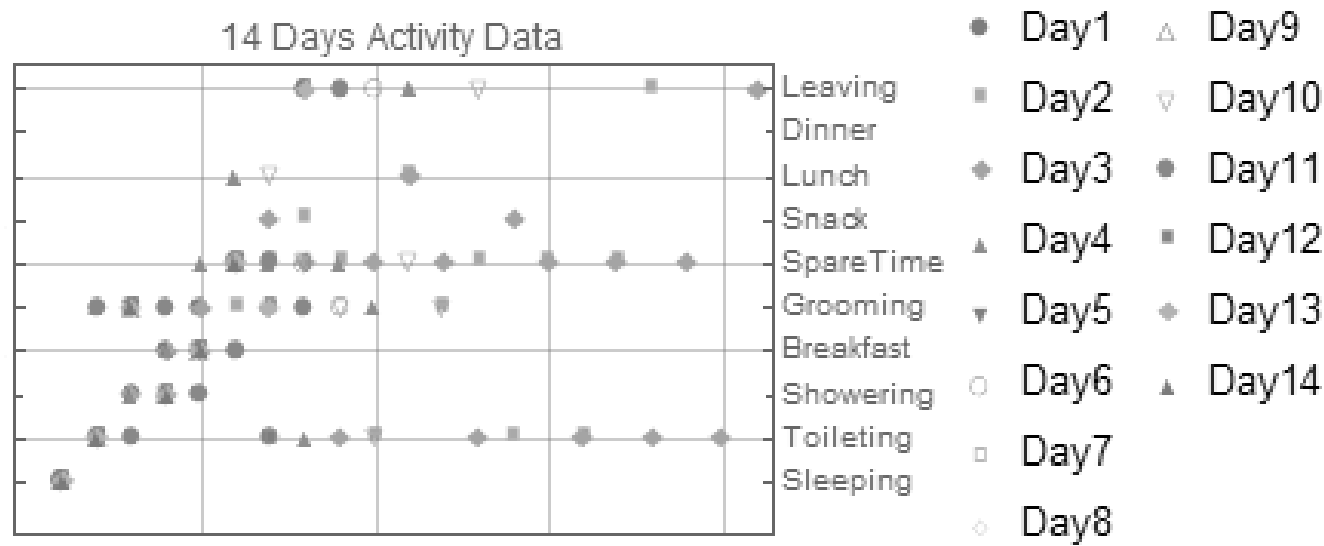

Figure 2: Activities Dataset Graph.

\subsection{HAR modelling (HAM)}

HAM refers to the modelling of the behaviour or activity of the person. Behaviour is regarded as an activity with duration, i.e. the time elapsed from start to end of an activity and time of day [14]. For example, a behaviour can be having breakfast, which consist of opening the refrigerator, cooking, sitting and eating breakfast. This set of activities are given in a time span (time elapsed from start to end), and usually in the morning (time of day).

Normal and abnormal activity and behaviour can be detected in a smart house by analysing both, the activity and the time. Abnormal activity detection main purpose is to warn a member of the family or caretaker whether something is wrong with the person. This can be regarded as anomaly. "Anomaly detection refers to the problem of finding patterns in data that do not conform to expected behavior" [15].

\section{Experiments}

The dataset used in this study is available in a text file only. Therefore, the dataset was exported to an excel file. The dataset contains 14 days of data in total. Two files were created, one for training and one for testing. Random numbers was generated in MATLAB with the randperm function to randomly select seven days for training. The numbers selected according to the random generator were days: 12, 10, 5, 14, 1, 7, and 6. Hence, these days were use for training. The remaining days $(2,3,4,8,9,11,13)$ were used for testing.

A new variable called duration was added. The vari- able duration was calculated using the time data from the dataset and consist of the time spent in each activity, from start to end of each activity. The duration value was calculated in seconds.

In the excel file, the text values of the dataset were coded to numbers. The variables room, position and activities were coded as explained in Section 2.3. The room values were coded to numbers from 1 to 5 . The position values coded to numbers from 1 to 3 . The $a c$ tivity values were coded to numbers from 1 to 9 .

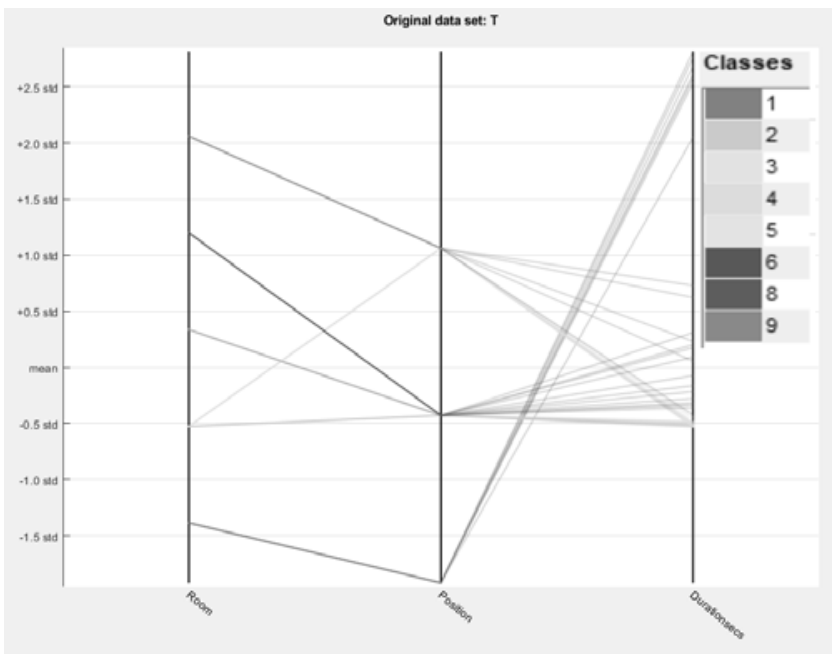

Figure 3: Parallel coordinated plots.

Both excel files were imported to MATLAB as table data type. The training was performed using the integrated classification learner toolbox. The variables used for training the decision trees are the room, position, and duration. The output variable is the activity 


\begin{tabular}{|c|c|c|c|c|}
\hline Date & StartTime & EndTime & Activity & Room \\
\hline $28-11-11$ & $02: 27: 59$ & 10:18:11 & Sleeping & Bedroom \\
\hline $28-11-11$ & $10: 21: 24$ & $10: 23: 36$ & Toileting & Bathroon \\
\hline $28-11-11$ & $10: 25: 44$ & 10:33:00 & Showering & Bathroon \\
\hline $28-11-11$ & $10: 34: 23$ & 10:43:00 & Breakfast & Kitchen \\
\hline 28-11-11 & 10:49:48 & 10:51:13 & Grooming & Bathroon \\
\hline $28-11-11$ & 10:51:41 & 13:05:07 & $\begin{array}{l}\text { Spare } \\
\text { Time }\end{array}$ & $\begin{array}{l}\text { Living- } \\
\text { room }\end{array}$ \\
\hline $28-11-11$ & 13:06:04 & 13:06:31 & Toileting & Bathroon \\
\hline 28-11-11 & 13:09:31 & 13:29:09 & Leaving & Hall \\
\hline 28-11-11 & 13:38:40 & $14: 21: 40$ & $\begin{array}{l}\text { Spare } \\
\text { Time }\end{array}$ & $\begin{array}{l}\text { Living- } \\
\text { room }\end{array}$ \\
\hline 28-11-11 & $14: 22: 38$ & 14:27:07 & Toileting & Bathroon \\
\hline 28-11-11 & 14:27:11 & 15:04:00 & Lunch & Kitchen \\
\hline 28-11-11 & 15:04:59 & 15:06:29 & Grooming & Bathroon \\
\hline 28-11-11 & 15:07:01 & 20:20:00 & $\begin{array}{l}\text { Spare } \\
\text { Time }\end{array}$ & $\begin{array}{l}\text { Living- } \\
\text { room }\end{array}$ \\
\hline $28-11-11$ & $20: 20: 55$ & 20:20:59 & Snack & Kitchen \\
\hline 28-11-11 & 20:21:15 & 02:06:00 & $\begin{array}{l}\text { Spare } \\
\text { Time }\end{array}$ & $\begin{array}{l}\text { Living- } \\
\text { room }\end{array}$ \\
\hline
\end{tabular}

Table 2: Day 1 example of the dataset.

data.

Figure 3 shows the parallel coordinated plots of the data. The variables room, position, and duration are plotted to show the relationship between them. According to Figure 3, it is possible to see that activities 3 and 5 (showering and grooming) follow almost the same path line in the graph. Also, activities 4 and 8 (breakfast and lunch) almost follow the same path line, with the duration barely different for each of the two activities. Activity 7, snack, was not found in the training dataset.

Once the tree is trained, testing is performed with the remaining seven days of the dataset: days $2,3,4$, $8,9,11,13$. The testing consists on using the variables room, position, and duration as input data. The response or output is the activity value. Each day from the testing dataset was tested and compared to the real data.

A new fictional test set was created in order to test the model with abnormal data, as showed in Table 6 . The test set consists of a fictional single day. The table shows that the duration of some of the activities were exaggerated. In addition, the position: lying of the first

\begin{tabular}{l|l}
\hline Name of Room & Number Assigned \\
\hline Bedroom & 1 \\
\hline Bathroom & 2 \\
\hline Kitchen & 3 \\
\hline Livingroom & 4 \\
\hline Hall & 5 \\
\hline
\end{tabular}

Table 3: House rooms and their code.

\begin{tabular}{l|l}
\hline Name of Activity & Number Assigned \\
\hline Sleeping & 1 \\
\hline Toileting & 2 \\
\hline Showering & 3 \\
\hline Breakfast & 4 \\
\hline Grooming & 5 \\
\hline Spare time/TV & 6 \\
\hline Snack & 7 \\
\hline Lunch & 8 \\
\hline Leaving & 9 \\
\hline
\end{tabular}

Table 4: Activities numbers and their code.

activity in the hall room should qualify as abnormal behaviour.

Result plots were obtained using the Mathematica software. The actual data and the predicted data for each of the testing days was copied to Mathematica and plots were coded to visually present the results.

Finally, the total computational time was measured.

\section{Results}

Figure 4 shows the trained decision tree. The decision tree model was able to classify seven out of nine activities in the dataset.

Figure 5 shows the number of observations for each of the activities. The true class is in the y-axis and the predicted class is in the $\mathrm{x}$-axis. It is possible to see that there are no observations in the training dataset for activity number seven (snack), and only one observation for activity number eight (lunch).

Most error counts in Figure 5 occurred in activities that are performed in the same room, such as showering and grooming (3 and 5). However, this number of 


\begin{tabular}{l|l}
\hline Name of Activity & Number Assigned \\
\hline Lying & 1 \\
\hline Sitting & 2 \\
\hline Standing & 3 \\
\hline
\end{tabular}

Table 5: Position numbers and their code.

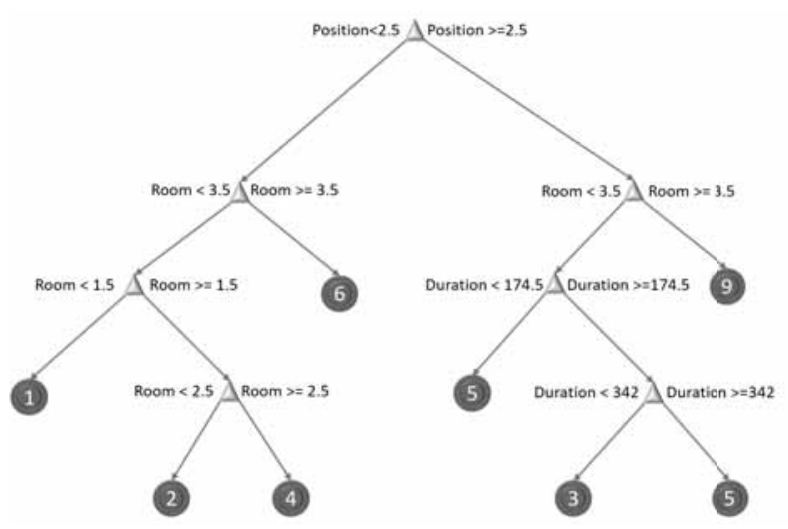

Figure 4: Trained Decision Tree. Red circles represent the activities.

observations with errors is low.

Figure 6 shows in percentage of success and errors prediction in the training data, called "positive predictive values false discovery rate". The highest false discovery is 50\% in the activity grooming (5). The model classified the activities showering (3) half of the times instead of the true class grooming (5).

For the activity grooming (5), the model had a false discovery rate of $22 \%$, classifying the activity showering (3) instead of grooming (5).

For the activity lunch (8), the model classified the activity breakfast (4) with a false discovery of $13 \%$.

Figure 7 shows the results of the test. Days 2, 3, 4,

\begin{tabular}{l|l|l|l}
\hline Room & Position & Durationsecs & Activity \\
\hline Hall & Lying & 10000 & - \\
\hline Bathroom & Sitting & 15000 & toileting \\
\hline Bathroom & Standing & 450 & grooming \\
\hline Living-room & Sitting & 9000 & spare time \\
\hline Living-room & Sitting & 9500 & spare time \\
\hline Hall & Standing & 412 & leaving \\
\hline
\end{tabular}

Table 6: Added fictional test day.

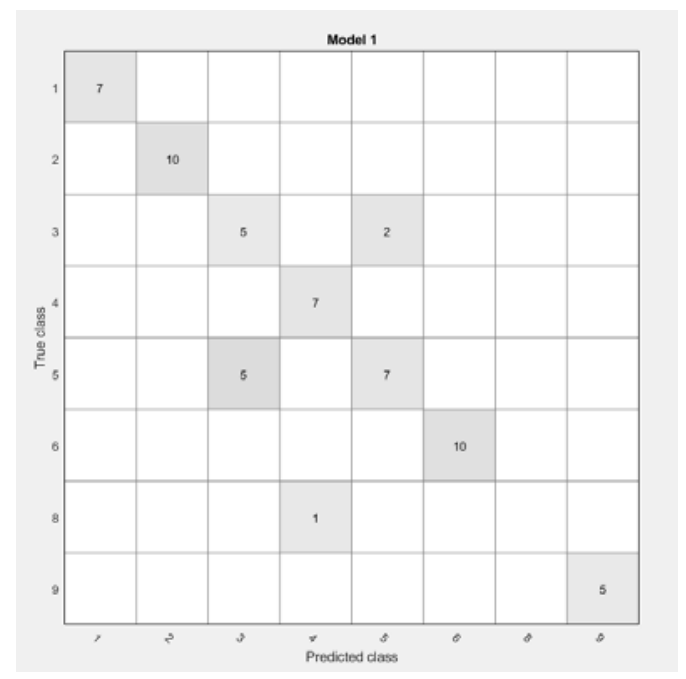

Figure 5: Number of observations.

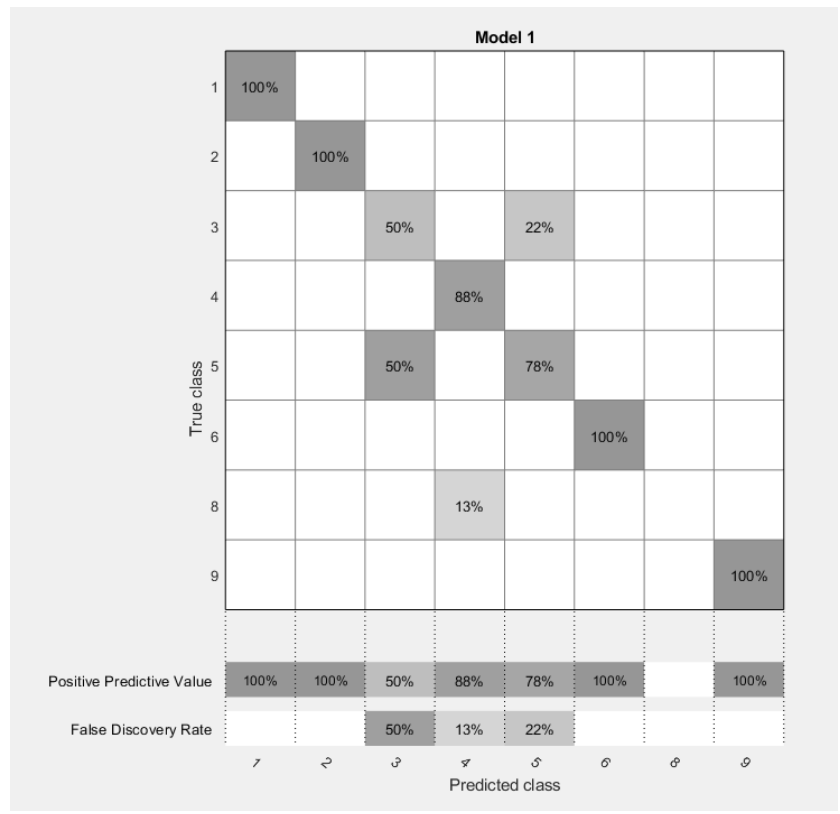

Figure 6: Positive predictive values and false discovery rates.

$8,9,11$, and 13 were used for testing. Some prediction errors were found when comparing the actual data with the estimated data.

Most of the errors were found between the grooming and showering activity, and between the breakfast and snack activities. A possible explanation for these prediction errors is that both of these activities are performed in the same room, bathroom and kitchen, respectively.

Figure $7 \mathrm{~h}$ shows the results of the added fictional 
day with abnormal behaviour data. The model predicted the activity Spare Time instead of finding an abnormal behaviour in the first activity.

The true positive-false positive rate for each fo the predicted activities are shown in Table 7.

Finally, the total accuracy of the activity recognition task is $88.02 \%$. The computational time of the model, consisting of training and testing is around 3 seconds.

\begin{tabular}{l|c|c}
\hline Activity & True Positive & False Positive \\
\hline Sleeping & $100 \%$ & 0 \\
\hline Toileting & $100 \%$ & 0 \\
\hline Showering & $77 \%$ & $23 \%$ \\
\hline Breakfast & $98 \%$ & $2 \%$ \\
\hline Grooming & $94 \%$ & $6 \%$ \\
\hline Spare time & $100 \%$ & 0 \\
\hline Lunch & $36 \%$ & $64 \%$ \\
\hline Leaving & $100 \%$ & 0 \\
\hline
\end{tabular}

Table 7: True positive, false positive rate for each activity.

\subsection{Test on second dataset}

The model was tested on the second dataset (OrdonezB) consisting of 21 days, also open source as described in Section 2.3. The purpose of this second test is to verify that the model works with any dataset.

In this test, the entire dataset was used, without sampling. The dataset was also processed as described in Section 3 . The values were coded to numbers. A total of 10 activities, 5 rooms and 3 positions were used.

The results showed that the model worked as well as in the experimental work (dataset Ordonez A). Like in the experimental work, minor mistakes were found in the prediction task corresponding to activities made in the same room. Namely bathroom and kitchen. Therefore, the model presented here is able to work with any dataset.

\section{Discussion}

In this work, decision trees are researched to perform human activity recognition modelling.

The decision tree classified seven out of nine activities. This is because there are no observation of activity snack, and only one observation for activity lunch in the training dataset. Therefore, the model could only classify seven activities in total.

Some predictions presented minor error rates. One possible reason for the these error rates is that there are rooms that allow different types of activities. Hence, the recognition task is more difficult. For example, in the room bathroom, three different activities are performed: showering, toileting and grooming. Thus, the model tends to predict the highest probability of the activity given the room bathroom.

This is the same case for the room kitchen, where activities breakfast, lunch and snack are performed. The activity lunch has an error rate of $64 \%$. From Figure 5 it is possible to see that there is only one observation of the activity lunch. Therefore, the model would hardly predict this activity. Instead, the model predicts the activity breakfast, because it has the highest probability.

Decision trees are probabilistic algorithms and thus produces some errors in the prediction task. As any probabilistic algorithm, decision trees will always chose the highest probability according to the trained data.

In general, the finding suggest that decision trees are a good tool for HAR with $88.02 \%$ accuracy. However, for the HAM, the model does not detects abnormal behaviour as well as it does HAR. When a fictional single day test set was created to check the performance on an abnormal day, the model did not meet the expectations in the anomaly detection task.

The most probable reason for this is that decision trees do not always enforce to check every variable before estimating a results. Consequently, in the added fictional test day with room hall and position lying, the tree predicted the activity spare time. Thus, the tree does not check for yhe other variables of room, nor $d u$ ration. The model should have detected an abnormal behaviour in this scenario, since lying in the hall is not a normal activity, but a possible fall.

Another reason for the model not detecting abnormal behaviour is that abnormal situations need to be trained in decision trees. This means that all possible abnormal scenarios need to be learned a priori. As a result, the findings suggest that decision trees are not the best option for detecting abnormal activities or behaviour.

The model was also tested with the second dataset available (OrdonezB) to verify that the model is able to work with any dataset. The results obtained were similar to the experimental work. Minor mistakes were 


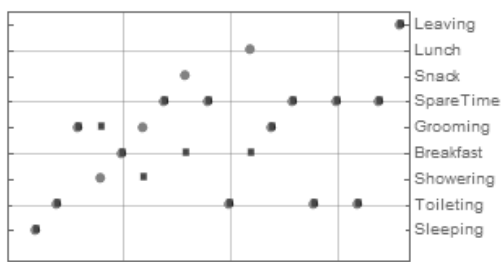

(a) Day 2 Results.

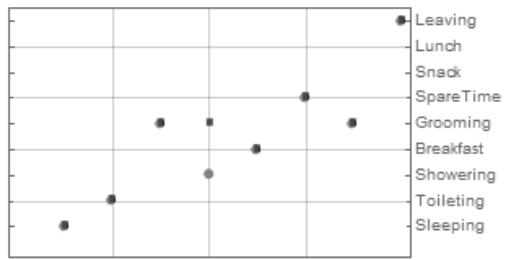

(d) Day 8 Results.

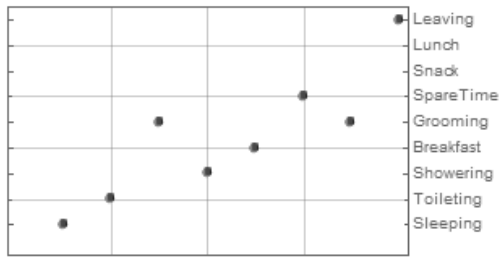

(g) Day 13 Results.

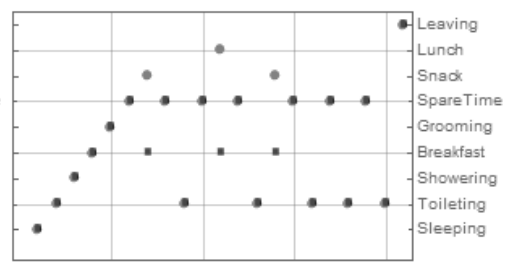

(b) Day 3 Results.

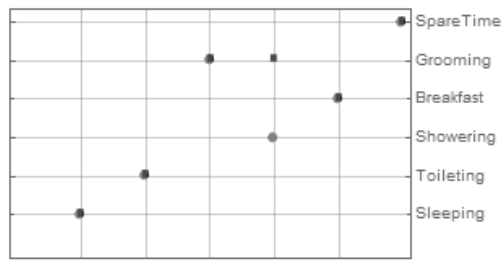

(e) Day 9 Results.

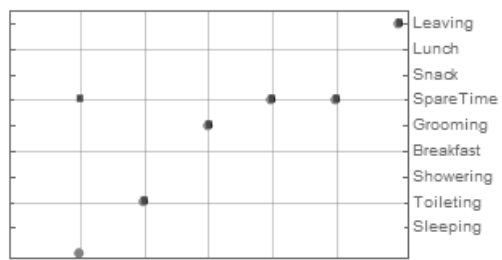

(h) Added fictional day results.

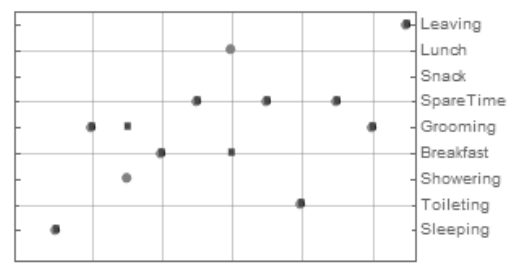

(c) Day 4 Results.

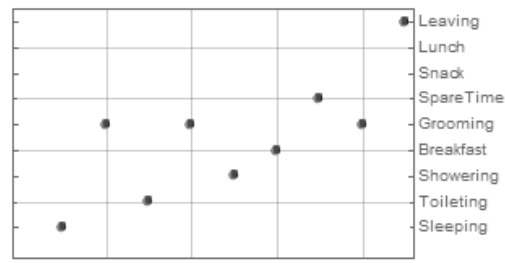

(f) Day 11 Results.

\section{- Actual Data \\ - Estimated Data}

Figure 7: Results.

found in activities performed in the same room.

Possible solutions for improving the model are more research on how to enforce the decision trees to check every single parameter. Another option could be to combine decision trees with another probabilistic method to increase the accuracy of the model.

Finally, HAM would ideally keep the activity history of the user in order to model the behaviour of the person. For example, if the user has followed the pattern wake up, toileting, grooming, showering and breakfast, the most normal behaviour would be not to repeat any of those activities again within a given frame time.

\section{Ethics in Smart Houses}

Smart house technology, like any other type of technology, can carry many ethical challenges. Therefore, a separate study has been carried at USN to address this topic. We consider that the ethical aspects are an important part of our research in smart house technology.

Among the main challenges found that smart houses present are cost-effectiveness, privacy, autonomy, in- formed consent, dignity, safety, and trust [16].

These challenges are central to keep in mind when developing a smart house system. Developers need to be aware of these challenges in order to provide a safer and dignified environment for the users. Nevertheless, it is important to acknowledge that smart house systems, at some point, cannot solve all the problems that are related to ageing, disabilities and diseases. There are needs that people develop as they age and smart house technology cannot help them any more [16].

\section{Conclusion and Future Work}

In this study, activity recognition modelling (HAM) is researched. The goal is to find the normal and abnormal behaviour of the person living in a smart house. Decision trees have been used to perform activity recognition because they can predict responses to data. The output from the activity recognition task is used as an input for the modelling task.

The input data for the decision trees learning task are the rooms, duration and position. The responses are 
the activities. A total accuracy of $88.02 \%$ was achieved for activity recognition using decision trees. Thus, decision trees can be a good tool for activity recognition. However, HAM did not meet the expected results.

The possible reason for this is that decision trees do not enforce to verify every single input variable before calculating a result. Therefore, more research on how to check every variable before estimating a results needs to be studied. Alternatively, combining decision tree algorithm with another probabilistic model could be a possible solution for HAM.

\section{References}

[1] Reaz MBI. Artificial Intelligence Techniques for Advanced Smart Home Implementation. Acta Technica Corviniensis-Bulletin of Engineering. 2013;6(2):51.

[2] Vainio AM, Valtonen M, Vanhala J. Proactive fuzzy control and adaptation methods for smart homes. Intelligent Systems, IEEE. 2008;23(2):42-49.

[3] Chan M, Estève D, Escriba C, Campo E. A review of smart homes-Present state and future challenges. Computer methods and programs in biomedicine. 2008; 91(1):55-81.

[4] Alam M, Reaz M, Ali M, Samad S, Hashim F, Hamzah M. Human activity classification for smart home: A multiagent approach. In: Industrial Electronics \& Applications (ISIEA), 2010 IEEE Symposium on. IEEE. 2010; pp. 511-514.

[5] Bourobou STM, Yoo Y. User Activity Recognition in Smart Homes Using Pattern Clustering Applied to Temporal ANN Algorithm. Sensors. 2015; 15(5):11953-11971.

[6] sentralbyraa S. Key figures for the population, 2017. https://www.ssb.no/en/befolkning/nokkeltall/population. 2018. Accessed 2017-12-10.

[7] Sanchez VG, Pfeiffer CF, Skeie NO. A Review of Smart House Analysis Methods for Assisting Older People Living Alone. Journal of Sensor and Actuator Networks. 2017;6(3):11.

[8] McBurney S, Papadopoulou E, Taylor N, Williams H. Adapting pervasive environments through machine learning and dynamic personalization. In: 2008 IEEE International Symposium on Parallel and Distributed Processing with Applications. IEEE. 2008; pp. 395-402.

[9] Bao L, Intille SS. Activity recognition from user-annotated acceleration data. In: Pervasive computing, pp. 1-17. Springer. 2004;.

[10] Fan X, Huang H, Xie C, Tang Z, Zeng J. Private smart space: Cost-effective ADLs (Activities of Daily
Livings) recognition based on superset transformation. In: Ubiquitous Intelligence and Computing, 2014 IEEE 11th Intl Conf on and IEEE 11th Intl Conf on and Autonomic and Trusted Computing, and IEEE 14th Intl Conf on Scalable Computing and Communications and Its Associated Workshops (UTC-ATC-ScalCom). IEEE. 2014; pp. 757-762.

[11] Lara OD, Labrador MA. A survey on human activity recognition using wearable sensors. IEEE Communications Surveys and Tutorials. 2013; 15(3):1192-1209.

[12] Ordónez FJ, de Toledo P, Sanchis A. Activity recognition using hybrid generative/discriminative models on home environments using binary sensors. Sensors. 2013;13(5):5460-5477.

[13] Ordonez. Activities of Daily Living (ADLs) Recognition Using Binary Sensors Data Set. 2013. Accessed: 2017-05-01.

URL https :

//archive.ics.uci.edu/ml/datasets/ Activitiestof+Daily+Living+28ADLs29+ Recognition+Using+Binary+Sensors

[14] Pfeiffer CF, Sánchez VG, Skeie NO. A Discrete Event Oriented Framework for a Smart House Behavior Monitor System. In: Intelligent Environments (IE), 2016 12th International Conference on. IEEE. 2016; pp. 119-123.

[15] Chandola V, Banerjee A, Kumar V. Anomaly detection: A survey. ACM computing surveys (CSUR). 2009; 41(3):15.

[16] Sánchez VG, Taylor I, Bing-Jonsson PC. Ethics of Smart House Welfare Technology For Older Adults: A Systematic Literature Review. International Journal of Technology Assessment in Health Care. 2017;pp. 1--9. 


\title{
Benefit Analysis of Wind Energy Storage by Time Shift Simulation
}

\author{
Daniela Andor ${ }^{1}$, Arturo Aquino ${ }^{1,2}$, Eduardo Martínez-Cámara ${ }^{1}$, \\ Juan Ignacio Latorre ${ }^{3}$, Emilio Jiménez ${ }^{1 *}$
}

\begin{abstract}
${ }^{1}$ University of La Rioja, High Technical School of Industrial Engineering. Departments of Electrical Engineering and Mechanical Engineering, San José de Calasanz 31, 26004 Logroño (La Rioja). Spain; *emilio.jimenez@unirioja.es

¿Universidad de Huelva, Escuela Técnica Superior de Ingeniería, Department of Electronic Engineering, Computer Systems and Automatics, Spain; arturo.aquino@diesia.uhu.es

${ }^{3}$ Public University of Navarre, Department of Mechanical, Energy and Materials Engineering, Campus of Tudela, 31500 Tudela (Navarre), Spain; juanignacio.latorre@unavarra.es
\end{abstract}

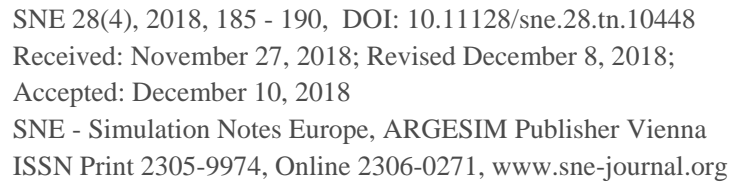

SNE 28(4), 2018, 185 - 190, DOI: 10.11128/sne.28.tn.10448 Received: November 27, 2018; Revised December 8, 2018;

Accepted: December 10, 2018

SNE - Simulation Notes Europe, ARGESIM Publisher Vienna ISSN Print 2305-9974, Online 2306-0271, www.sne-journal.org

Abstract. Currently there exist several energy storage technologies that are suitable for wind energy integration services. Energy prices in several countries (such as in Spain) are set for the day ahead market, which means the hourly prices are known the day before. This represents an opportunity for wind power plant owners. Wind energy generated in hours when demand and prices are the lowest could be stored and sold in hours when demand and prices are higher. This paper analyses the benefit of wind energy storage by time shift depending on climatological (wind), technological (storage facilities), and market (power prices) factors for the Spanish case, as exemplification of a methodology to be used in any other country. Wind energy time shift has been simulated for periods of time of 1 hour up to 9 hours considering two scenarios, a day with low wind energy generation and a day with high wind generation, in order to determine in which moments a beneficial exists with the different energy storage technologies. According to the results, on a day with high wind energy levels the gain obtained by time shifting wind energy from low to high demand hours could reach $68,1 \%$, and on a day with low wind energy levels, the gain obtained by time shifting wind energy could reach $19,3 \%$.

\section{Introduction}

Currently there exist several energy storage technologies that are suitable for wind energy integration services. Energy storage can make wind energy more reliable and economically more attractive in an environmentally responsible way. Through the strategic utilization of storage, the use of renewable energy, and more specifically the use of wind energy, can in fact be improved.
The energy storage applications depend on the operational requirements and the duration and frequency of discharge. Possible value streams of energy storage include flexible capacity, energy arbitrage, system balancing and ancillary services, congestion management, renewable time shifting, forecast hedging, emissions and power quality. Energy storage allows for the improved management of energy supply and demand and can provide multiple valuable energy and power sources.

The relation between wind energy and demand curve is also very important. Wind is more similar to load than to conventional generation: wind and most loads are nondispatchable resources, they have cycling behaviour, and they depend on weather conditions and sometimes deviate from the forecast. Energy storage can also be used to mitigate wind power curtailment.

In several counties the price of electricity ( $€ / \mathrm{MWh})$ is lower when demand is lower which gives an opportunity for economic benefits by storing wind energy when demand is low and selling it during peak demand when prices are higher. This is the case in Spain, whose power system (wind power and electricity demand) is used to exemplify this methodological proposal.

The study is based on the technical characteristics and performance parameters, system costs, benefits and drawbacks, environmental impact, and commercial status of pumped hydro storage, compressed air energy storage, hydrogen fuel cell storage systems, flywheel energy storage systems and batteries and ultracapacitors. Also several battery energy storage technologies including lead acid, nickel cadmium, nickel metal hydride, lithium-ion, sodium sulphur, vanadium redox flow, zinc bromide, zinc chlorine, iron redox, sodium nickel chloride and nickel iron are considered. 
This paper is also based on a previous work of the authors [1] that analysed in detail the power rating, discharge time, energy and power density, efficiency, life time and cycle life, environmental impact and capital cost of the energy storage technologies, as well as the technical and ecumenical suitability of the different energy storage technologies for certain services and applications based on their specific technical requirements.

With this base, the time shift of $1 \mathrm{MWh}$ of energy storage for a duration of 1 to 9 hours has been simulated using energy storage technologies. According to the results, on a day with high wind energy levels the gain obtained by time shifting wind energy from low to high demand hours could reach $68,1 \%$. On a day with low wind energy levels, the gain obtained by time shifting wind energy could reach $19,3 \%$.

Thus, as a summary, the paper analyses the benefit of wind energy storage by time shift depending on climatological (wind), technological (storage facilities), and market (power prices) factors for the Spanish case, as a methodology to be used in any other case. The particularities of wind generation and wind energy grid integration are analysed in section 1. Section 2 is devoted to Energy Storage, presenting a brief Energy Storage Technology Overview and analysing the Characteristics of the energy storage systems. In section 3 wind energy time shift has been simulated considering two scenarios, a day with low wind energy generation and a day with high wind generation, in order to determine in which moments a benefit can be achieved with the different energy storage technologies. Finally section 4 summarizes the conclusions of the simulation-based analysis.

\section{Wind Energy}

Wind energy is expected to be the cornerstone and driving force for the immediate application of a world energy system driven by renewable energy to completely substitute fossil and nuclear sources [2]. This work, through summarization of a research on optimally harnessing wind energy by means of energy storage, demonstrates that indeed wind energy is a front-runner in the process of clean energy transformation.

\subsection{Wind energy generation}

Wind energy is considered as a clean and competitive renewable energy resource. In developing countries, wind energy has already a significant share in the energy mix. In developing and emerging countries, wind energy is one of the main focus in the efforts made to respond to climate change and diversify the energy mix.
Wind power is one of the most popular and fast expanding sources of renewable energy; the worldwide installed wind capacity is expected to reach $631 \mathrm{GW}(602$ GW onshore) by 2020 according to [3]. Spain is one of the five traditional wind countries together with China, USA, Germany and India representing a $72 \%$ share of the global wind capacity. In fact, the total capacity installed in Spain by 2016 was 23057 MW [4].

\subsection{Wind energy integration}

Wind energy is intermittent and weather and location dependent. These characteristics of wind energy create challenges for wind integration in power systems.

Wind speed is not constant, making electricity output uncontrollable. Electricity output variations are not perceptible in the range of seconds to minutes. In one day, however, the cumulative wind energy production at country level can ramp from near maximum to near zero and vice versa. This translates in additional energy required to match supply and demand immediately.

Wind resource is partially unpredictable. For the grid, this means maintaining reserves for additional power when wind energy generation produces less than expected. Dispatchable loads must also be available at times when wind generation produces in excess.

Another characteristic of wind energy is that generation must be located where the best wind resources are. These locations are often far from the places where the power will be consumed. Connecting wind power to the grid requires most times new transmission capacity.

Wind power variability and the impacts that it has on power systems have been studied in [5], which states that wind power integration impacts on power systems depend on the level of flexibility the systems have and on the wind penetration level. The general impacts identified in this study are generation efficiency (it affects other conventional generation units), necessity for back-up reserves, curtailed wind energy, reliability, transmission and distribution losses and voltage and reactive power.

In [6], the authors studied the potential challenges of integrating wind energy in power systems focusing on social impacts, economic impacts, environmental impacts and technical impacts.

Power systems that take in large amounts of wind power need to be flexible to deal with the variability and uncertainty. The needed flexibility could be achieved with grid friendly wind energy generation, improved flexibility in conventional generation, transmission expansion, operational enhancement and demand response. Energy storage can be an important player in adding flexibility as it can act both as generation and load [7-9]. 


\section{Energy Storage}

\subsection{Energy storage technology overview}

One of the characteristics of electricity is that it cannot be stored directly, requiring continuously balancing with the demand, this resulting in costly implications. Sufficient generating capacity is required to match the highest demand level, even though the capacity increment is only needed for short periods of time and infrequently [10, 11].

Also, due to the inability to store electricity, reserve generating capacity must be maintained available as spinning or non-spinning reserves for potential changes in the load or the unplanned loss of a generation plant [10].

Although it is not possible to store electricity directly, it can be converted to other forms of energy that can be stored. The stored energy is converted to electricity when needed. Energy storage is a fundamental intermediary between variable energy generation sources and variable loads. Without it, energy generation is forced to equal consumption. The greatest advantage of energy storage is that it is capable of moving energy through time.

From the electrical system standpoint, the energy storage systems act as loads during storage and as sources of electricity when returning energy to the system [10]. Electrical energy can be stored in energy forms such as mechanical, chemical and electro-chemical, electromagnetic, and thermal (this one not interesting for this study) [12,10]:

i. Mechanical energy storage. Mechanical energy storage technologies are pumped hydroelectric storage (PHS) [13], compressed air energy storage (CAES) and flywheel energy storage. In PHS the energy is stored as potential gravitational energy while in CAES, the energy is stored as potential pressure energy. Flywheel energy storage consists of storing energy in the form of rotational kinetic energy. PHS and CAES are considered technologies suited for energy management whereas flywheels are more suitable for power applications.

ii. Chemical energy storage. Hydrogen energy storage is a chemical storage technology. Hydrogen is used as an energy carrier for electricity storage through a process such as electrolysis [14].

iii. Electrochemical energy storage. Electrochemical energy storage technologies convert electricity in chemical energy during charging. Batteries are considered electrochemical energy storage systems. Power converter interfaces for electrochemical energy storage systems have been reviewed in [15]. Innovation in electrochemical energy storage technologies has been monitored in [16].

iv. Electrical field storage. Capacitors are considered electrical field storage systems. Capacitors consist of two electrical conductors separated by a dielectric material (non-conducting). The electric field is created when applying voltage across the conductors causing opposite electrical charges build up on the conductors. The surface area of the conductors and the distance between them determined the capability of energy storage. In general capacitors consist of two plates separated by a thin dielectric. The capacitors can be electrostatic, electrolytic and electrochemical. The difference between electrostatic and electrolytic capacitors is that the electrolytic uses a liquid electrolyte as one of the plates.

v. Magnetic energy storage. This form of energy storage consists in storing energy in a magnetic field. The flow of direct current in a super-cooled coil creates a magnetic field capable of storing energy. Superconducting magnetic energy storage (SMES) is an example of magnetic field storage technology. In SMES, the magnetic energy can be stored indefinitely once the superconducting coil is charged. Cryogenic refrigeration is required to maintain the magnetic coil so cool as to have superconducting properties $[10,14]$.

\subsection{Characteristics of energy storage systems}

The potential of energy storage technologies for the demands of different applications is determined by their characteristics. This subsection defines classical properties according to the existing literature.

The characteristics of energy storage technologies such as discharge duration, power rating, energy storage capacity, response time and costs in the context of benefits determine the applications they are most suited for. The criteria for the selection of energy storage technology are divided into 4 aspects [1]:

- Design: Power rating, Storage capacity, Discharge duration, Response time, Energy density per unit area, Energy density per unit volume, Technology maturity, Reliability, Modularity, Siting requirements, Portability, Synergies with other energy applications.

- Operation: Overall cycle, Efficiency, Lifetime/maximum number of charge-discharge cycles, Parasitic losses.

- Financial: Capital cost per energy stored, Capital cost per power rating, cost for Fixed O\&M, Variable O\&M, Replacement, Disposal, and Commercial risk.

- Other aspects: Health and safety aspects, Environmental impacts, Synergies with other sectors.

The ideal characteristics of storage technologies would be low capital and low operating and maintenance cost, have a long life and high efficiency, be flexible in operation and have a fast response and be environmentally friendly. The main characteristics for choosing energy storage have been defined in [17] and [10]. 


\section{WindEnergy Time ShiftSimulation}

The problem with renewable generation is that it often does not match demand. Peak demand and renewable generation typically occur at different times in the day and year. Renewable energy generation is also dependent on weather and geographic conditions. When generation exceeds the need for serving load, energy storage could be used to store energy for times when generation in not enough to serve the load. Storage helps removing time specific constraints [10].

Energy prices in Spain are set for the Day Ahead Market (DAM), which means the hourly prices are known the day before. This represents an opportunity for wind power plant owners $[4,5,6]$. Wind energy generated in hours when demand and prices are the lowest could be stored and sold in hours when demand and prices are higher. Wind energy time shift has been simulated considering two scenarios, a day with low wind energy generation and a day with high wind generation. The level of wind energy generation influences energy prices. When wind energy generation levels are high, the prices are lower and vice versa.

The low wind day selected for the time shift simulation is May 19, 2015 (see Figure 1). The lowest hourly price registered was $34.40 € / \mathrm{MWh}$ between 4:00 and 5:00 am. The day with high wind energy generation levels selected for the wind energy time shift simulation is May 05, 2015 (see Figure 2). On the day with high wind energy generation levels, the minimum price registered during low demand hours was $14.95 € / M W h$ (between 3:00 and 4:00 am) while the highest price was $62 € / \mathrm{MWh}$ (between 21:00 and 22.00 pm).

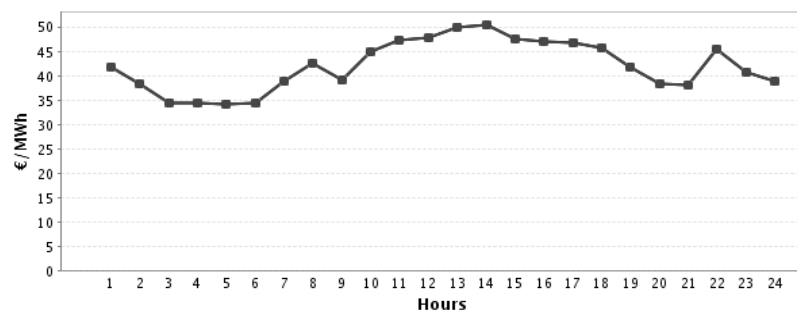

Figure 1. Hourly marginal prices in Spain and Portugal (case of day with low wind, 19/05/2015).

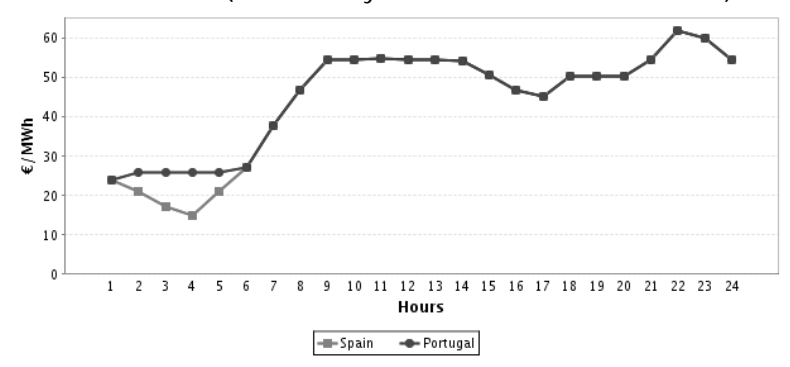

Figure 2. Hourly marginal prices in Spain and Portugal (case of day with high wind, 05/05/2015).
The energy storage technologies considered in the simulation are CAES, PHS, Hydrogen Fuel Cell Storage Systems (HFCSS), Lead Acid (LA) batteries, NiCd batteries, Li-ion batteries, NaS batteries, Vanadium Redox Battery (VRB), Zinc-Bromine flow Batteries (ZBB) and Zero Emissions Batteries Research Activity (ZEBRA) batteries. Flywheel Energy Storage (FES) systems and ultracapacitors (UC) have been discarded due to their short duration storage (below 1 hour) and high selfdischarge rates (hourly self discharge of FES is higher than 20\%).

Based on the findings in the existing literature regarding energy storage system (ESS) cost, round trip efficiency (RTE), storage duration and self discharge, the following assumption were made (see Table 1), obtained from a deep analysis developed in [1].

These technical characteristics of the energy storage systems will for sure improve with the advance of the technology, but the methodology presented in this paper will be still valid, just adapting the study with the new values. The technical and economic feasibility of storing $1 \mathrm{MWh}$ of wind energy for several hours has been studied. The economic merit of time shift consists in the difference between energy purchase cost, store and discharge energy cost.

\subsection{High wind day scenario}

The time shift of $1 \mathrm{MWh}$ of wind energy for one or several hours has been made considering the hourly prices registered on May 05, 2015. The lowest price per MWh on that day was registered at 3:00 am. The price escalated continuously until 10:00 am when it reached 55.08 $€ / M W h$. The gain achieved by time shifting $1 \mathrm{MWh}$ of wind energy for up to 7 hours using different energy storage technologies can be seen in Table 2, where it can be seen that the difference between the lowest price and the highest price goes from $29 \%$ up to $73 \%$.

\begin{tabular}{|l|c|c|c|c|}
\hline ESS & RIE & $\begin{array}{c}\text { Cost } \\
\text { ( } / \mathbf{M W h})\end{array}$ & $\begin{array}{c}\text { Self-discharge } \\
\text { (\% hour) }\end{array}$ & $\begin{array}{c}\text { Storagedur } \\
\text { ration }\end{array}$ \\
\hline CAES & $71 \%$ & 0,05 & $0 \%$ & $2-50 \mathrm{~h}$ \\
\hline PHS & $75 \%$ & 0,3 & $0 \%$ & h-days \\
\hline HFCSS & $40 \%$ & 0,01 & $0 \%$ & h-days \\
\hline FES & $90 \%$ & 3 & $20 \%$ & $<1 \mathrm{~h}$ \\
\hline LA & $80 \%$ & 0,35 & $0,01 \%$ & min-days \\
\hline Ni-Cd & $85 \%$ & 1,5 & $0,02 \%$ & $1-8 \mathrm{~h}$ \\
\hline Li-ion & $90 \%$ & 2,5 & $0,17 \%$ & m-days \\
\hline NaS & $85 \%$ & 0,45 & 0 & $10 \mathrm{~h}$ \\
\hline VRB & $75 \%$ & 1 & $0,42 \%$ & s- $12 \mathrm{~h}$ \\
\hline ZBB & $75 \%$ & 0,5 & $0,00 \%$ & h-months \\
\hline ZEBRA & $85 \%$ & 0,65 & $0,63 \%$ & hours \\
\hline UC & $95 \%$ & 10 & $0,83 \%$ & ms-1h \\
\hline
\end{tabular}

Table 1: Assumptions regarding the energy storage technologies considered in the time shift simulation. 


\begin{tabular}{|c|c|c|c|c|c|c|c|c|}
\hline $\begin{array}{c}\text { Hour of } \\
\text { the day }\end{array}$ & $\begin{array}{c}\mathbf{0 3 -} \\
\mathbf{0 4}\end{array}$ & $\begin{array}{c}\mathbf{0 4 -} \\
\mathbf{0 5}\end{array}$ & $\begin{array}{c}\mathbf{0 5 -} \\
\mathbf{0 6}\end{array}$ & $\begin{array}{c}\mathbf{0 6 -} \\
\mathbf{0 7}\end{array}$ & $\begin{array}{c}\mathbf{0 7 -} \\
\mathbf{0 8}\end{array}$ & $\begin{array}{c}\mathbf{0 8 -} \\
\mathbf{0 9}\end{array}$ & $\begin{array}{c}\mathbf{0 9 -} \\
\mathbf{1 0}\end{array}$ & $\begin{array}{c}\mathbf{1 0 -} \\
\mathbf{1 1}\end{array}$ \\
\hline $\mathbf{f} \mathbf{M M h}$ & $\mathbf{1 4 , 9 5}$ & $\mathbf{2 1 , 0 0}$ & $\mathbf{2 7 , 1 3}$ & $\mathbf{3 7 , 7}$ & $\mathbf{4 7 , 0 2}$ & $\mathbf{5 4 , 6}$ & $\mathbf{5 4 , 7 4}$ & $\mathbf{5 5 , 0 8}$ \\
\hline $\begin{array}{c}\text { Hours of } \\
\text { storage }\end{array}$ & 0 & 1 & 2 & 3 & 4 & 5 & 6 & 7 \\
\hline CAES & & $-0,5$ & 22,2 & 44,1 & 55,2 & 61,4 & 61,5 & 61,7 \\
\hline PHS & & 3,7 & 25,7 & 46,7 & 57,3 & 63,3 & 63,4 & 63,6 \\
\hline HFCSS & & $-78,1$ & $-37,8$ & 0,8 & 20,5 & 31,5 & 31,7 & 32,1 \\
\hline LA & & 9,5 & 30,2 & 50,0 & 59,9 & 65,5 & 65,6 & 65,8 \\
\hline Ni-Cd & & 9,8 & 31,3 & 51,4 & 61,3 & 66,9 & 66,9 & 67,1 \\
\hline Li-ion & & 9,9 & 32,2 & 52,5 & 62,4 & 67,9 & 67,9 & $\mathbf{6 8 , 1}$ \\
\hline NaS & & 14,4 & 34,1 & 52,8 & 62,2 & 67,5 & 67,6 & 67,8 \\
\hline VRB & & $-0,4$ & 22,9 & 45,0 & 56,0 & 62,2 & 62,1 & 62,2 \\
\hline ZBB & & 2,8 & 25,1 & 46,4 & 57,2 & 63,2 & 63,2 & 63,5 \\
\hline ZEBRA & & 12,6 & 32,5 & 51,6 & 61,2 & 66,6 & 66,4 & 66,4 \\
\hline
\end{tabular}

Table 2: Time shift simulation results for high wind day scenario in time period 03h-11h (in \%).

The following conclusions can be drawn regarding the energy price and time of storage (Table 2):

- Time shifting $1 \mathrm{MWh}$ of wind energy for 1 hour reported economical gain with all the energy storage technologies except for CAES, HFCSS and VRB due to low RTE efficiencies or higher cost of the storage system. An important factor influencing the gain is the considerable energy price difference (29\% higher).

- After 2 hours of storage and an increase of energy price of $45 \%$, only the time shift with HFCSS does not provide gain. This is due to the low round trip efficiency of the system (40\%). During the following hours, all the energy storage technologies used for time shift report benefits.

- The energy price difference increases significantly over the 7 hours: $45 \%$ (3 hours); $68 \%$ (4 hours), $73 \%$ (5, 6 and 7 hours). The gain obtained by time shifting $1 \mathrm{MWh}$ of wind energy increases as the energy price increases. The highest gain is obtained with the Liion battery energy storage reaching $68,1 \%$.

- Between 16:00 and 17:00 pm on the same day (May 05,2015 , energy price was $45.25 € / \mathrm{MWh}$ and it reached $62 € / \mathrm{MWh}$ at 21:00 pm. A time shift simulation was made given the considerable price difference.

In Table 3 it can be seen that the energy price difference is much lower compared to the first time period simulated. The price is steady for the first three hours with a difference of $10 \%$ compared to the lowest price. The fourth hour the difference reached $17 \%$ while the fifth hour the difference is $27 \%$. As it can be seen, the price difference is not enough for the first three hours to produce economical gain by time shifting $1 \mathrm{MWh}$ of wind energy.
Only Li-ion and NaS battery produce economical gain with an energy price difference of $17 \%$. This is due to their high round trip efficiencies.

With an energy price difference of $27 \%$, except for CAES, HFCSS and VRB, time shifting $1 \mathrm{MWh}$ of wind energy with the rest of energy storage technologies provides economic benefit. The highest gain is achieved with Li-ion battery energy storage.

\subsection{Low wind day scenario}

Time shifting of $1 \mathrm{MWh}$ of wind energy has been simulated for a day with low wind energy. The low wind day chosen is May 19, 2015. Energy prices are higher, and the energy price difference registered in this day with low wind is considerably smaller (32\%) compared to the difference registered in the high wind day (73\%).

The lowest energy price registered on May 19, 2015 was at 5:00 am while the highest was registered 9 hours later at 13:00 pm. The gain achieved by time shifting 1 MWh of wind energy for up to 9 hours using different energy storage technologies can be seen in Table 4 .

From the results it can be seen that time shift does not report benefit with any of the energy storage technologies with small energy price differences ( $1 \%$ to $12 \%)$. When energy price increases by $19 \%$ ( 3 hours), time shifting with NiCd, Li-ion, NaS and ZEBRA batteries produces economic benefit. NiCd batteries have maximum storage duration of 8 hours. Time shift with HFCSS does not report benefits at all due to the very low round trip efficiency.

\begin{tabular}{|c|c|c|c|c|c|c|}
\hline $\begin{array}{c}\text { Hour of } \\
\text { the day }\end{array}$ & $\mathbf{1 6 - 1 7}$ & $\mathbf{1 7 - 1 8}$ & $\mathbf{1 8 - 1 9}$ & $\mathbf{1 9 - 2 0}$ & $\mathbf{2 0 - 2 1}$ & $\mathbf{2 1 - 2 2}$ \\
\hline $\mathbf{f / M M h}$ & $\mathbf{4 5 , 2 5}$ & $\mathbf{5 0 , 5 0}$ & $\mathbf{5 0 , 5}$ & $\mathbf{5 0 , 5}$ & $\mathbf{5 4 , 7 4}$ & $\mathbf{6 , 0 0}$ \\
\hline $\begin{array}{c}\text { Hours of } \\
\text { storage }\end{array}$ & $\mathbf{0}$ & $\mathbf{1}$ & $\mathbf{2}$ & $\mathbf{3}$ & $\mathbf{4}$ & $\mathbf{5}$ \\
\hline CAES & & $-26,3$ & $-26,3$ & $-26,3$ & $-16,5$ & $-2,9$ \\
\hline PHS & & $-20,2$ & $-20,2$ & $-20,2$ & $-10,8$ & 2,2 \\
\hline HFCSS & & $-124,1$ & $-124,1$ & $-124,1$ & $-106,7$ & $-82,5$ \\
\hline LA & & $-12,8$ & $-12,8$ & $-12,8$ & $-4,0$ & 8,2 \\
\hline Ni-Cd & & $-8,7$ & $-8,7$ & $-8,7$ & $-0,1$ & 11,9 \\
\hline Li-ion & & $-4,9$ & $-5,1$ & $-5,2$ & 3,3 & $\mathbf{1 5}$ \\
\hline NaS & & $-6,4$ & $-6,4$ & $-6,4$ & 1,9 & 13,5 \\
\hline VRB & & $-22,4$ & $-22,8$ & $-23,1$ & $-13,6$ & $-0,5$ \\
\hline ZBB & & $-20,7$ & $-20,7$ & $-20,7$ & $-11,2$ & 1,9 \\
\hline ZEBRA & & $-7,4$ & $-7,9$ & $-8,4$ & $-0,2$ & 11,2 \\
\hline
\end{tabular}

Table 3: Time shift simulation results for high wind day scenario in time period $16 \mathrm{~h}-22 \mathrm{~h}$ (in \%). 


\begin{tabular}{|c|c|c|c|c|c|c|c|c|c|c|}
\hline $\begin{array}{l}\text { Hour of } \\
\text { the day }\end{array}$ & $\begin{array}{l}5- \\
66\end{array}$ & $\begin{array}{l}06- \\
07\end{array}$ & $\begin{array}{l}07- \\
08\end{array}$ & $\begin{array}{l}08- \\
09\end{array}$ & $\begin{array}{l}09- \\
10\end{array}$ & $\begin{array}{l}10- \\
11\end{array}$ & $\begin{array}{c}11- \\
12\end{array}$ & $\begin{array}{l}12- \\
13\end{array}$ & $\begin{array}{l}13- \\
14\end{array}$ & $\begin{array}{l}14- \\
15\end{array}$ \\
\hline €/MWh & $\begin{array}{c}34, \\
4\end{array}$ & $\begin{array}{l}34, \\
60\end{array}$ & 39 & $\begin{array}{l}42, \\
71\end{array}$ & $\begin{array}{l}39, \\
21\end{array}$ & $\begin{array}{c}45, \\
1\end{array}$ & $\begin{array}{c}47, \\
4\end{array}$ & 48 & $\begin{array}{c}50, \\
2\end{array}$ & $\begin{array}{c}50, \\
6\end{array}$ \\
\hline & 0 & 1 & 2 & 3 & 4 & 5 & 6 & 7 & 8 & 9 \\
\hline CAES & & $-40,2$ & 24,4 & $-13,6$ & $-23,7$ & $-7,5$ & $-2,3$ & $-1,0$ & 3,4 & 4,2 \\
\hline & & 33,7 & $-18,5$ & $-8,2$ & $-17,9$ & $-2,4$ & 2,6 & 3,8 & 8,1 & 8,8 \\
\hline HFCSS & & 48 & -120 & -101 & -119 & $-90,7$ & $-81,5$ & $-79,2$ & $-71,3$ & $-70,0$ \\
\hline & & $-25,6$ & $-11,3$ & $-1,5$ & $-10,7$ & 3,9 & 8,6 & 9,7 & 13,7 & $\mid 14,4$ \\
\hline $\mathrm{i}-\mathrm{Cc}$ & & $-22,3$ & $-8,0$ & 1,7 & $-7,4$ & 7,1 & 11,7 & 12,8 & 16,8 & \\
\hline Li-ion & & $-19,3$ & $-5,1$ & 4,4 & $-4,9$ & 9,5 & 14,0 & 14,9 & 18,7 & 19,3 \\
\hline & & $-18,5$ & $-5,0$ & 4,2 & $-4,4$ & 9,4 & 13,8 & 14,9 & 18,7 & \begin{tabular}{|l|}
19,3 \\
\end{tabular} \\
\hline VRB & & $-37,1$ & $-21,7$ & $-11,4$ & $-22,1$ & $-6,3$ & $-1,4$ & $-0,6$ & 3,5 & 3,8 \\
\hline 3B & & $-34,5$ & $-19,1$ & $-8,7$ & $-18,5$ & $-2,8$ & 2,2 & 3,4 & 7,7 & 8,4 \\
\hline ZEBRA & & 20,0 & $-6,9$ & 1,9 & $-7,7$ & 6,0 & 10,0 & 10,6 & 14,0 & 14,1 \\
\hline
\end{tabular}

Table 4: Time shift simulation results for low wind day scenario in time period 05h-15h (in \%).

\section{Results}

After simulating the time shift of $1 \mathrm{MWh}$ wind energy for periods of time of 1 hour up to 9 hours in a low wind day scenario and a high wind day scenario using several energy storage technologies including CAES, PHS, HFCSS, LA, Ni-Cd, Li-ion, NaS, VRB, ZBB and ZEBRA batteries, the following can be concluded:

i) In the scenario with high wind energy levels, time shift with energy storage reported gains up to $68,1 \%$.

ii) In the scenario with low wind energy, time shift with energy storage reported gains up to $19,3 \%$.

iii) All the selected storage technologies are technically suitable for this service in terms of storage duration and selfdischarge. Ni-Cd has storage duration up to 8 hours. The hourly selfdischarge of the batteries is not significant; however it affects the possible gain when energy is stored for several hours.

iv) Energy price is the most important factor in terms of benefit. The difference between the energy price during low demand and the energy price during high demand hours determines the economic feasibility of the time shift service.

v) The efficiency and cost of the energy storage system are very important. Low energy efficiency and high system costs affect the possible gain that can be obtained with time shift. When energy price variation in time is low, low round trip efficiencies and high storage system costs make this service economically unfeasible.

\section{References}

[1] Andor D. Energy storage systems for wind energy integration. Technology, applications, and benefit analysis, $\mathrm{PhD}$ Thesis, 2015, University of La Rioja https://dialnet.unirioja.es/servlet/tesis?codigo $=46779$
[2] W.W.E.A. World Wind Resource Assessment Report. 2014.

[3] (IEA) I.E.A. Medium Term Renewable Energy Market Report 2014 - Market Analysis and Forecast to 2020. 2014.

[4] El sistema eléctrico español 2016 (pdf, translated as "The Spanish electrical system 2016"). Red Eléctrica de España (trsnalated as "Spanish Electrical Network"). https://www.ree.es/sites/default/files/11_PUBLICACIONES/Documentos/InformesSistemaElectrico/2016/inf_sis_elec_ree_2016.pdf

[5] Albadi MH, El-Saadany EF. Overview of wind power intermittency impacts on power systems. Electric Power Systems Research, 2010. 80(6): p. 627-632.

[6] Shafiullah GM, et al. Potential challenges of integrating largescale wind energy into the power grid-A review. Renewable and Sustainable Energy Reviews, 2013. 20: p. 306-321.

[7] (IEC) I.E.C. Grid integration of large-capacity Renewable Energy sources and use of large-capacity Electrical Energy Storage. 2012.

[8] Solomon AA, Kammen DM., Callaway D, The role of largescale energy storage design and dispatch in the power grid: A study of very high grid penetration of variable renewable resources. Applied Energy, 2014. 134: p. 75-89.

[9] Crespo-Vazquez JL, Carrillo C, Diaz-Dorado E, MartinezLorenzo JA, Noor-E-Alam M. (2018). A machine learning based stochastic optimization framework for a wind and storage power plant participating in energy pool market. Applied Energy, 232, 341-357. doi:10.1016/j.apenergy.2018.09.195

[10] Rachel-Carnegie DG, Nderitu D, Preckel PV. Utility Scale Energy Storage Systems. Purdue University. State Utility Forecasting Group. 2013.

[11] Jani V, Abdi H. Optimal allocation of energy storage systems considering wind power uncertainty. Journal of Energy Storage, 20, 244-253. doi:10.1016/j.est.2018.09.017

[12] Zhao H, et al. Review of energy storage system for wind power integration support. Applied Energy, 2015. 137: p. 545-553.

[13] Saad Y, Younes R, Abboudi S, Ilinca A. Hydro-pneumatic storage for wind-diesel electricity generation in remote sites. Applied Energy, 231, 1159-1178. doi:10.1016/j.apenergy.2018.09.090

[14] (IEA) I.E.A. Technology Roadmap Energy storage. 2014.

[15] Fernão-Pires V, et al. Power converter interfaces for electrochemical energy storage systems - A review. Energy Conversion and Management, 2014. 86: p. 453-475.

[16] Müller S, Sandner P, Welpe I, Monitoring Innovation in Electrochemical Energy Storage Technologies: A Patent-based Approach. Energy Procedia, 2014. 61: p. 2293-2296.

[17] Ibrahim H, Ilinca A, Perron J. Energy storage systemsCharacteristics and comparisons. Renewable and Sustainable Energy Reviews, 2008. 12(5): p. 1221-1250.

[18] Díaz G, Coto J, Gómez-Aleixandre J. Optimal operation value of combined wind power and energy storage in multi-stage electricity markets. Applied Energy, 235, 1153-1168. doi:10.1016/j.apenergy.2018.11.035

[19] Gomes ILR, Melicio R, Mendes VMF, Pousinho HMI. Decision making for sustainable aggregation of clean energy in day-ahead market: Uncertainty and risk. Renewable Energy, 133, 692-702. doi:10.1016/j.renene.2018.10.054

[20] Jiang Y, Yu S, Wen B. Monthly electricity purchase and decomposition optimization considering wind power accommodation and day-ahead schedule. International Journal of Electrical Power and Energy Systems, 107, 231-238. doi:10.1016/j.ijepes.2018.11.001 


\title{
A Simulation-based Analysis of the Influence of Renewables on the Spanish Electricity Prices
}

\author{
Diego Azofra' ${ }^{1}$,Emilio Jiménez ${ }^{1 *}$, Eduardo Martínez-Cámara ${ }^{1}$, Julio Blanco ${ }^{1}$, \\ Juan Ignacio Latorre ${ }^{2}$ \\ ${ }^{1}$ University of La Rioja. High Technical School of Industrial Engineering. Departments of Electrical Engineering and \\ Mechanical Engineering. San José de Calasanz 31, 26004 Logroño (La Rioja). Spain; *emilio.jimenez@unirioja.es \\ 2Public University of Navarre, Department of Mechanical, Energy and Materials Engineering, Campus of Tudela, \\ 31500 Tudela (Navarre), Spain; juanignacio.latorre@unavarra.es
}

SNE 28(4), 2018, 191 - 196, DOI: 10.11128/sne.28.tn.10449 Received: November 27, 2018; Revised December 8, 2018;

Accepted: December 10, 2018

SNE - Simulation Notes Europe, ARGESIM Publisher Vienna ISSN Print 2305-9974, Online 2306-0271, www.sne-journal.org

Abstract. Spain is at the forefront of renewable energy worldwide with a great integration level in its generation mix. This has put Spain in the situation that other countries that support renewable energy will be in a short-medium term. However active measures to support renewable energy have been losing strength in recent times seeking to reduce the deficit. On the one hand, the bonus for each technology is different and, on the other hand, their integration level in the mix, and thus its influence on the Pool, is uneven. This work aims to provide information and conclusions about the actual economic impact of each renewable technology on the pool and the electricity tariff for the Spanish case. For doing so, simulation and artificial intelligence techniques are applied in order to model the energy pool and simulate the electricity prices in five fictitious scenarios that suppress out of the generation mix, respectively, wind, photovoltaic, biomass, small hydraulic, and solar thermal. This way, based on the simulation, and ex-post analysis can be developed in order to obtain conclusions and knowledge, useful for other countries as well as for future decision making in the Spanish power system.

\section{Introduction}

Spain is at the forefront of renewable energies worldwide, with a great integration level of them in its generation mix. This has put Spain in the situation which the other countries supporting renewable energies will be in a short-medium term. However active policies to support renewable energy have been losing strength in recent times, seeking to reduce the deficit. This fact, coupled with the significant rising experienced by the electricity bill lately, have made ordinary people and society in general to belief that renewables technologies are expensive for the system and constitute the main cause for that rising.

It is true that renewable generation technologies have not been, up to date, competitive enough to challenge the traditional thermoelectric power plants, which has made them earn bonuses from generation. But renewables have in turn benefits for the system as their ranking preference in the energy auction gets the most expensive technologies out of the pool, lowering thus the electricity prices. That works in practice in a very uneven way depending on the type of renewable energy. This happens because, on the one hand, the bonus for each technology is different and, on the other hand, their integration level in the mix, and thus its influence on the pool, is disparate.

This paper presents a global view of the effects of different renewable energy sources on the electricity pool [1] combining and complementing the analysis of the effects of five renewable sources: wind, photovoltaic, biomass, small hydraulic, and solar thermal. The ex-post economic analysis of photovoltaic power in the Spanish grid was analysed in [2] by modelling alternative scenarios. The analysis of wind power merit-order and feed-intariffs effect by a variability analysis of the Spanish electricity market was developed in [3], while [4] compared the influence of photovoltaic and wind power on the Spanish electricity prices by means of artificial intelligence techniques and [5] did a similar comparison of biomass, solar-thermal and small hydraulic power. Finally [6] presented the economical-environmental impact analysis of subsidised renewable energy sources for electricity (RES-E) in the Spanish system. Other works have continued with this analysis of the renewable energy policy, the impact of wind generation on the spot market 
electricity pricing, and the support policies and merit order effect, such as [7-9].

In this work artificial intelligence techniques (algorithm M5P) are applied in order to model the energy pool cassation process. Once, the model has been created, it is time to simulate the new electricity price in five fictional scenarios that suppress wind, photovoltaic, biomass, small hydraulic, and solar thermal, respectively, out of the generation mix. Taking into account the price reduction produced for renewables as well as the bonus received, it is determined, for each renewable technology, whether it is beneficial or in deficit for the system. To go a step further and extrapolate the influence of renewable energies over the pool to the tariff or charges to be paid by the final consumer, the low voltage most common tariff, known as TUR, is recalculated for the above-mentioned scenarios. From this study it will be concluded that, with the analysed Spanish renewable policy, wind, biomass and small hydraulic power were beneficial while the photovoltaic and solar thermal technologies were economically deficient for the system, as well as for the electricity tariff.

\section{Methodology and Scope}

The main objectives of this work are:

- Apply artificial intelligence techniques (M5P algorithm - tree model) for the simulation of the electricity matching process in the Pool which have proved really accurate.

- Determine the economic influence of the main renewable technologies (wind, solar, biomass, small hydraulic and solar thermal) on the price reduction in the Spanish Pool.

- Taking into account the price reduction produced for renewables as well as the bonus received, determine for each renewable technology, whether it is beneficial or in deficit for the system.

That is, we want to determine, using an ex-post analysis, the economic influence of the major renewable technologies eligible for bonus (wind, photovoltaic, biomass, small hydraulic and solar thermal) on the Spanish electricity grid.

For doing so, five fictional scenarios, which suppress the Spanish generation mix each of the aforementioned technologies respectively have been created:

- SCENARIO A: Real generation mix excluding generation from wind technology.

- SCENARIO B: Real generation mix excluding the generation from photo-voltaic technology.

- SCENARIO C: Real generation mix, excluding generation from biomass.

- SCENARIO D: Real generation mix, excluding generation from thermoelectric solar technology.

- SCENARIO E: Real generation mix, excluding generation from small-hydraulic technology.

The balance in the system is restored in the model by filling the gap of renewable generation in each scenario by combined cycle and coal thermal power plants, being the only ones capable of acting as back-up power plants. The establishment of the new equilibrium in the system will provide a new pool price lower than the original (real mix with all technologies).

\section{Simulation Model}

Once the scenarios have been defined, it is necessary to generate a model that simulates the matching of the price of energy, thus determining the inversely proportional relationship between the $\%$ of integration of renewables in the Pool and the price of the resulting energy [2-6].

In order to reduce the dispersion resulting from the dispersion chart of the pool's price and the level of integration in the renewable mix, it is imperative to carry out a multivariate analysis that takes into account parameters such as generation by technology, capacity available by technology ..., and that in turn throw light on such dispersion of points.

Several prediction algorithms have been tested using WEKA in order to determine its accuracy to predicting the final price of energy in the Pool, based on the attributes. Several ones have presented a similar adjustment and correlation factor with respect to the database (M5P, Metabagging Reptree, M5rules, and IBK lazy) [1]. The M5P algorithm has been chosen, which allows us to obtain hidden knowledge of the matching process thanks to the graphic visualization of the model tree.

Once a model that represents the Spanish electric Pool with sufficient precision is determined, the new resulting price is simulated in each scenario.

Taking into account that the bonus produces an increase in the cost of electricity tariff and their preference order in the electricity auction produces a drop in the energy prices matching in the Pool, it is considered that if the two factors are eliminated, the influence of each renewable technology on the system and the electricity tariff is determined. 


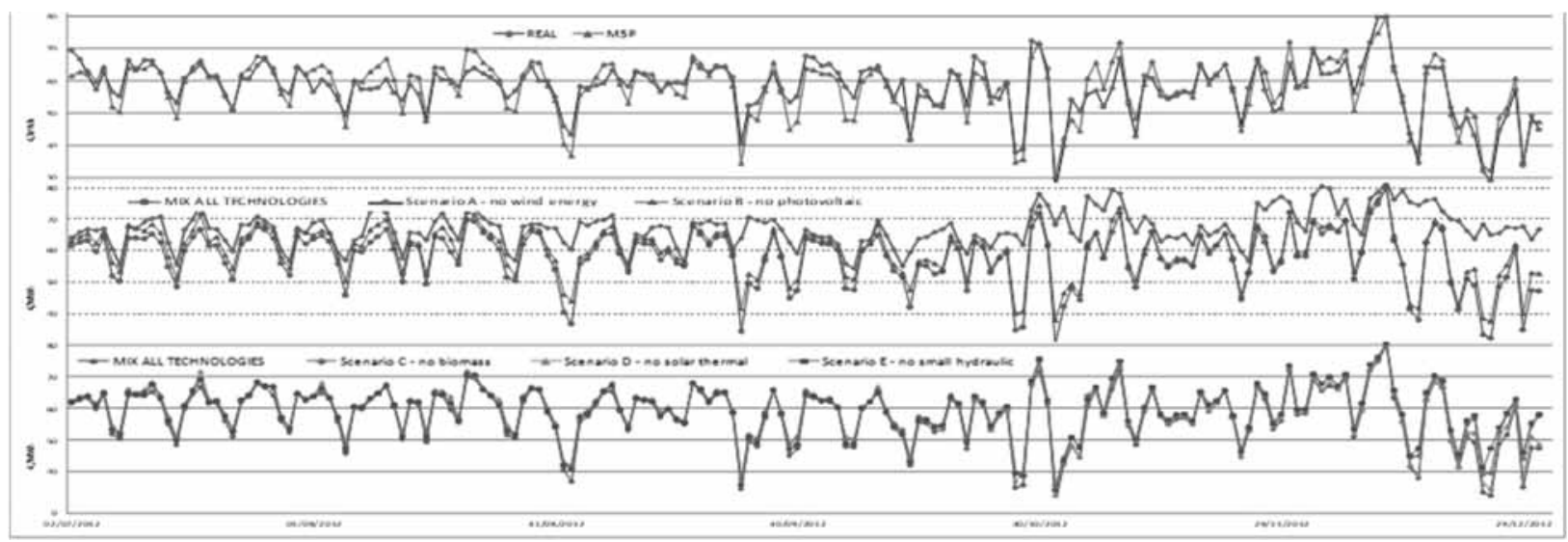

Figure 2. a) (upper graph) comparison between the final hourly price of real cassation (blue) and that obtained by the M5P prunned model (red), b) Comparison for each day of the final price obtained by the model for the scenario with all the technologies (red), scenario A without generation wind (blue) and B, without photovoltaic generation (green); c) comparison now with scenario C, without generation by biomass (blue), scenario D, without solar thermal generation (green) and scenario $\mathrm{E}$, without small-hydraulic generation (violet).

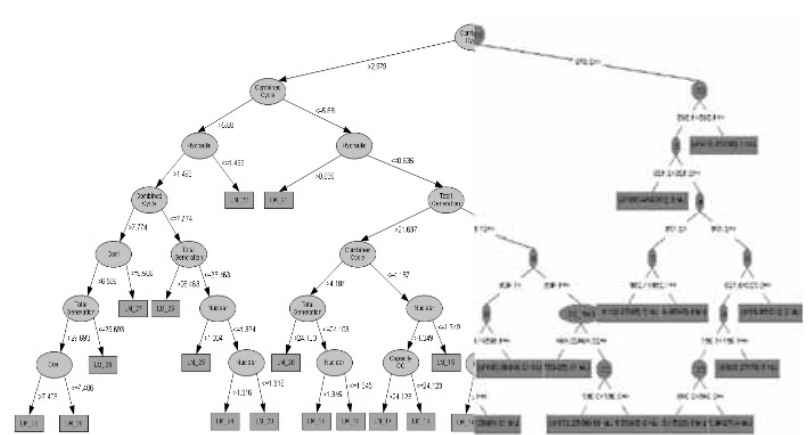

Figure 1. Model tree combining 2 different representation formats $[4,5]$.

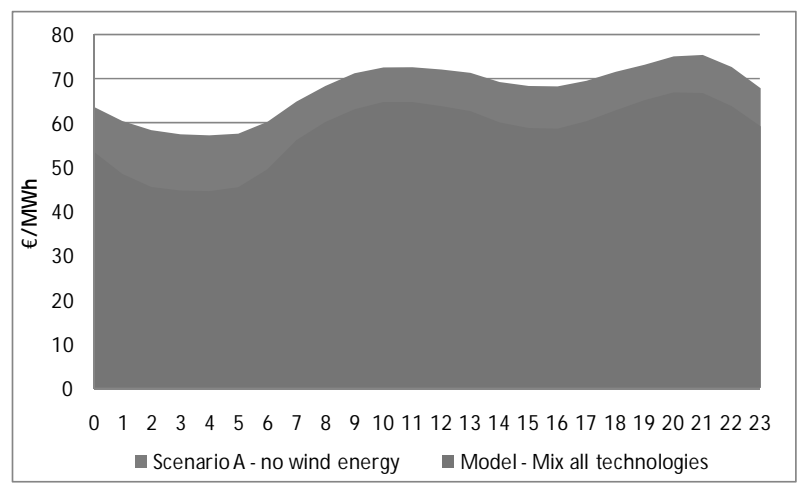

Figure 3. Final hourly prices obtained by the model for the real scenario vs. a scenario without wind generation.

The training database contains the schedule data of the variables shown in the previous point between January 1, and December 31, 2012 (the year selected for the ex-post analysis of this work). The calculations have been made by applying the cross-validation technique with an improvement of the holdout method.

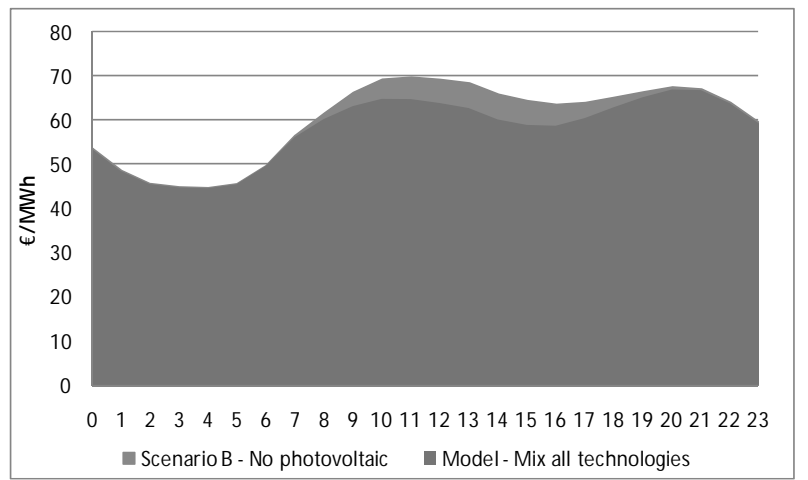

Figure 4. Final hourly prices obtained by the model for the real scenario vs. a scenario without photovoltaic generation.

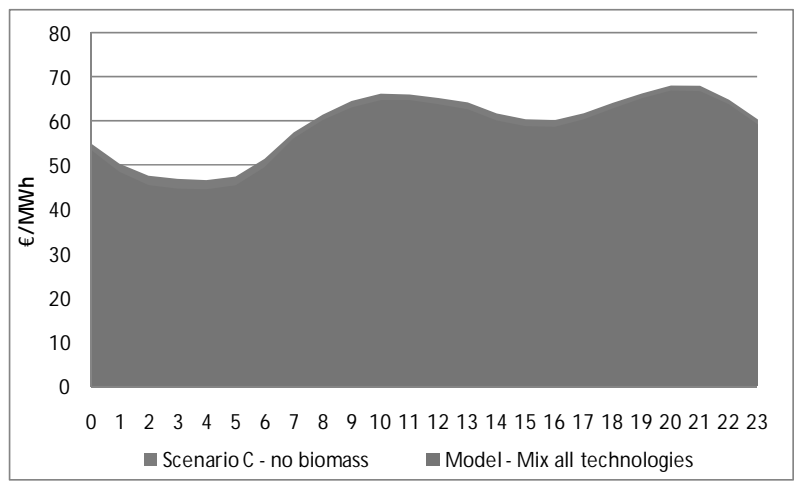

Figure 5. Final hourly prices obtained by the model for the real scenario vs. a scenario without biomass generation.

Once the model is trained, we have a model tree (Figure 1) that returns the MWh price based on the input variables used, and we will be able therefore to use it to determine the new pool price resulting in the five scenarios fictitious. 


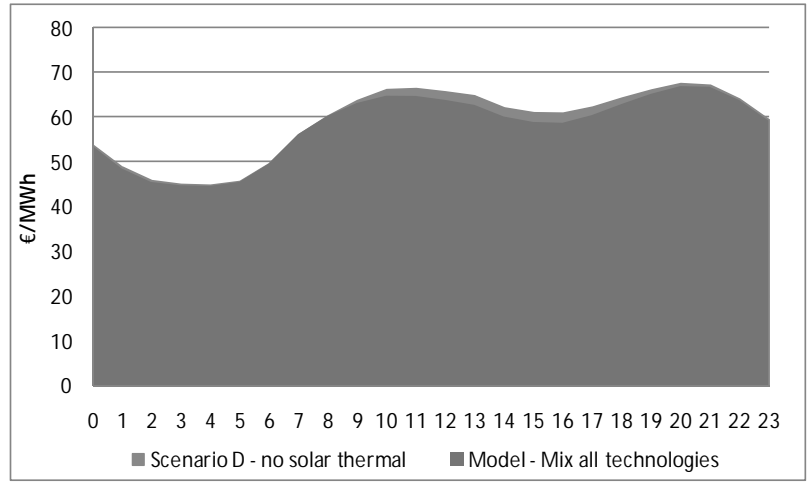

Figure 6. Final hourly prices obtained by the model for the real scenario vs. a scenario without solar thermal generation.

\section{Simulation Results: Economic Influence of Renewable Technologies}

Figure 2 represents the comparative [1] between the real case and the one obtained from the M5P algorithm (upper graph) and compares the hourly price of the electric power obtained by the model for the real case vs. the price obtained by the model for each of the scenarios for every day of a semester (wind and photovoltaic in the intermediate graph, and biomass, solar thermal and small hydraulic in the lower graph).

The results shown for a day are represented in figures 3 to 7 , which show that the scenarios without wind and photovoltaic generation return higher Pool matching prices. In relation to wind energy, the biggest difference is produced at night because demand decreases and wind generation remains relatively constant, which means that there is virtually no remaining generation to be covered by thermal power plants.

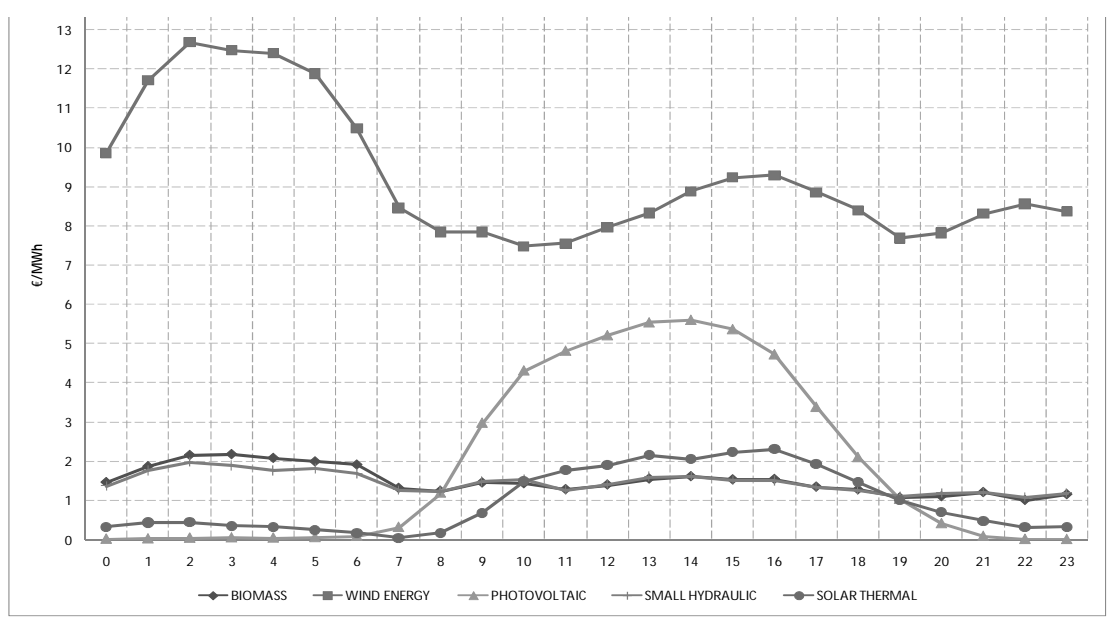

Figure 8. Decrease in the price of the energy produced by each technology per

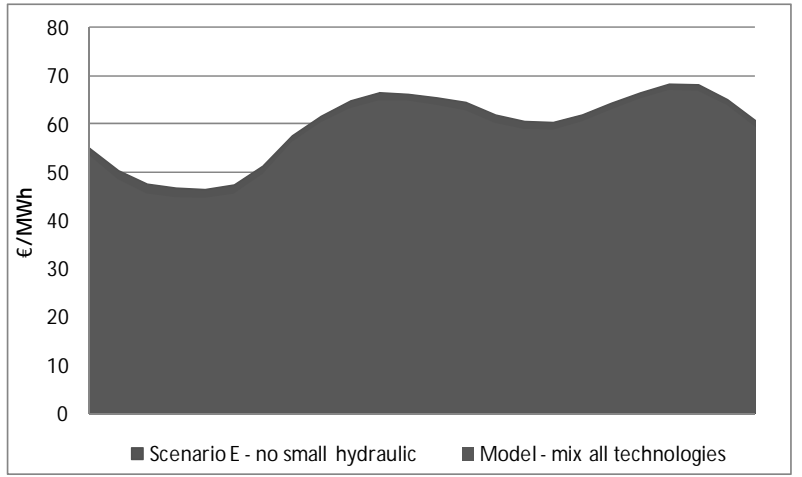

Figure 7. Final hourly prices obtained by the model for the real scenario vs. a scenario without small hydraulic generation.

With regard to photovoltaic energy, the generation curve during the day can be clearly observed, obtaining a maximum around 14:00 (12:00 in solar time in summer time) coinciding with the highest solar radiation over the country. The scenarios without electricity generation through biomass, solar thermoelectric or small hydraulic return higher Pool matching prices, which confirms again that the preference of order in the auction of the premium renewables generates a decrease in the final price of the auctioned energy. Figure 8 represents the decrease in the price of the energy produced by each technology individually for the day type.

\section{Conclusions}

Below are shown the main contributions made throughout this work as well as the results thereof.

i) The application of artificial intelligence techniques (Algorithm MP5 - model generation trees) to simulate the process of price cassation in the Pool, based on the levels of integration of each technology in the generation mix, have stated that:

- Wind power received bonuses for $€ 2037$ million, which caused a reduction of the average weighed price in the Pool of $9.10 € / \mathrm{MWh}$, generating as a whole a net saving for the grid of $€ 364.0$ million.

- Photovoltaic energy received bonuses of $€ 2610.7$ million, causing a reduction of the average weighed price in the Pool of $2.18 € / \mathrm{MWh}$, generating as a whole a net cost for the system of $€ 2034$.1 million. 
- The biomass for power generation received bonuses of $€ 344.0$ million, causing a reduction of the average weighed price in the Pool of $1.48 € / \mathrm{MWh}$, generating as a whole a net saving to the system of $€ 50.7$ million.

- Thermo-electric solar energy received bonuses of $€ 926.9$ million, causing a reduction of the average weighed price in the Pool of $1.05 € / \mathrm{MWh}$, generating as a whole a net cost for the system of $€ 648.2$ million.

- Energy from small-hydraulic power plants received bonuses of $€ 184.1$ million, causing a reduction of the average weighed price in the Pool of $1.45 € / \mathrm{MWh}$, generating as a whole a net saving for the system of $€ 200.6$ million.

ii) Going a step further and extrapolate the influence of renewable energies over the Pool to the tariff or charges to be paid by the final consumer in the year 2012, thus backing up the lack of related scientific literature up this moment, it has been concluded that:

- The wind power from the generation mix would have meant an extra cost for the average consumer in the TUR (Tariff of Last Resource) of $€ 1.95$ in the electricity bill. That is, wind power caused a $0.28 \%$ drop in the Tariff of Last Resource.

- To exclude photovoltaic energy from the generation mix would have meant a saving for the average consumer in the TUR of $€ 38.82$ in the electricity bill. That is, photovoltaic energy caused a $5.95 \%$ increase in the Tariff of Last Resource.

- To exclude biomass from the generation mix would have meant a cost for the average consumer in the TUR of $€ 0.12$ in the electricity bill. That is, biomass caused a $0.02 \%$ drop in the Tariff of Last Resource.

- To exclude solar thermo-electrical energy form the generation mix, would have meant a saving for the average consumer in the TUR of $€ 12.39$ in the electricity bill. That is, solar thermo-electric energy caused a $1.82 \%$ increase in the Tariff of Last Resource.

- To exclude the energy from small-hydraulic power plants from the generation mix would have meant an extra cost for the average consumer in the TUR of $€ 3.01$ in the electricity bill for the year 2012. That is, small-hydraulic energy caused a $0.43 \%$ drop in the Tariff of Last Resource.

iii) A sensitivity analysis allows a further knowledge of the effects of renewables depending on the actual penetration of the renewable source. For this purpose, 111 scenarios have been analysed, ranging from zero penetration in the grid to a $110 \%$ of the real wind power generation of the year 2012. The conclusions are:
- Energy generation by means of wind power technology would have been profitable to the grid as long as the generation level had been equal or higher than the of the $83 \%$ of the real generation.

- The worst wind power generation level, in economic terms, coincides with the $55 \%$ of the real generation. Under this scenario, the total cost overrun caused by wind power to the grid would have been of $€ 175.3$ million.

- The optimal wind power generation level, in economic terms, coincides with the $110 \%$ of the real generation. Under this scenario, the total saving produced by wind power over the system would have been of $€ 643.1$ million.

- Finally, and according to the variability of the wind map, if the year 2012 had been less windy, with a wind power generation of $90 \%$, the energy price reduction would have been of $7.42 € / \mathrm{MWh}$. On the contrary, if the year 2012 had been windier, with a generation of $110 \%$, the energy price reduction would have been of $10.94 € / \mathrm{MWh}$.

\section{Listings and Code}

The code used in this work, in concrete the tree model M5P and the VBA code for the sensitivity analysis of wind energy on the system, can be found on the Annex section of [1], which includes "Model tree - algorithm M5P”, "VBA code for sensitivity analysis of wind power on the system".

\section{References}

[1] Azofra D. Analysis of the influence of renewable energies on the price of electricity in Spain using Artificial Intelligence techniques/"Análisis de la influencia de las energías renovables sobre el precio de la electricidad en España mediante técnicas de Inteligencia Artificial", PhD Thesis, 2016, University of La Rioja https://dialnet.unirioja.es/servlet/tesis?codigo $=41546$

[2] Azofra D, Saenz-Díez JC, Martínez E, Jiménez E, Blanco J.). Ex-post economic analysis of photovoltaic power in the Spanish grid: Alternative scenarios. Renewable Energy, 95, 98-108, 2016. doi:10.1016/j.renene.2016.04.005.

[3] Azofra D, Jiménez E, Martínez E, Blanco J, SaenzDíez JC. Wind power merit-order and feed-in-tariffs effect: A variability analysis of the Spanish electricity market. Energy Conversion and Management, 83, 19-27. doi:10.1016/j.enconman.2014.03.057, 2014. 
[4] Azofra D, Martínez E, Jiménez E, Blanco J, Azofra F, Saenz-Díez JC. Comparison of the influence of photovoltaic and wind power on the Spanish electricity prices by means of artificial intelligence techinques. Renewable and Sustainable Energy Reviews, 42, 532-542. doi:10.1016/j.rser.2014.10.048.

[5] Azofra D, Martínez E, Jiménez E, Blanco J, SaenzDíez JC. Comparison of the influence of biomass, solar-thermal and small hydraulic power on the Spanish electricity prices by means of artificial intelligence techniques. Applied Energy, 121, 28-37, 2014. doi:10.1016/j.apenergy.2014.01.064.

[6] Azofra D, Jiménez E, Martínez E, Blanco J, SaenzDíez JC. Economical-environmental impact of subsidised renewable energy sources for electricity (RES-E) in the Spanish system. Energy for Sustainable Development, 29, 47-56, 2015. doi:10.1016/j.esd.2015.09.002.
[7] Blazquez J, Fuentes-Bracamontes R, Bollino CA, Nezamuddin $\mathrm{N}$. The renewable energy policy paradox. Renewable and Sustainable Energy Reviews, 82, 1-5, 2018. doi:10.1016/j.rser.2017.09.002

[8] Makalska T, Varfolomejeva R, Oleksijs R. The impact of wind generation on the spot market electricity pricing. Paper presented at the Proceedings - 2018 IEEE International Conference on Environment and Electrical Engineering and 2018 IEEE Industrial and Commercial Power Systems Europe, EEEIC/I and CPS Europe 2018, doi:10.1109/EEEIC.2018.8494539

[9] Lopes F, Sá J, Santana J. Renewable generation, support policies and the merit order effect: A comprehensive overview and the case of wind power in Portugal, 2018. doi:10.1007/978-3-319-74263-2_9 


\title{
Modeling and Validation of an Open-Source Mean Value Heavy-Duty Diesel Engine Model
}

\author{
Kristoffer Ekberg ${ }^{*}$, Viktor Leek, Lars Eriksson
}

Vehicular Systems, Department of Electrical Engineering, Linköping University, Sweden, 58183 Linköping; *kristoffer.ekberg@liu.se

SNE 28(4), 2018, 197-204, DOI: 10.11128/sne.28.tn.10451

Received: November 27, 2018; Revised December 10, 2018; Accepted: December 12, 2018

SNE - Simulation Notes Europe, ARGESIM Publisher Vienna, ISSN Print 2305-9974, Online 2306-0271, www.sne-journal.org

Abstract. The pursuit of lower fuel consumption and stricter emission legislation has made a simulation- and optimization-based development methodology important to the automotive industry. The keystone in the methodology, is the system model. But for the results obtained using a model to be credible, the model has to be validated. The paper validates an open-source, meanvalue engine model of a 13 liter $\mathrm{Cl}$ inline 6 cylinder heavyduty engine, and releases it as open-source.

\section{Introduction}

In today's automotive industry, there is a drive for lower fuel consumption and better control of emissions. Simulation and numerical optimization are two tools that can be used to achieve that. The keystone in a simulation driven approach, is the system model. For it (and the results obtained using it) to be credible, it needs to be validated. This paper presents the model structure of an open-source engine model and validates it.

The engine is a 13 liter CI inline 6 cylinder Scania engine. The model structure is a mean-value engine model (MVEM) [5], this type of structure does not model the piston movement explicitly. Instead the mean flow in and out of the cylinders is modeled. This makes the model computationally efficient, and suitable for control and optimization of the air and fuel path of the engine. It has a low number of states for efficient simulations, and is continuously differentiable for use with gradient-based optimization solvers.

The model is a continuation of previous modeling work by the authors, described in [1]. In contrast to the previous work, indicated mean effective pressure
(IMEP), pump mean effective pressure (PMEP) and friction mean effective pressure (FMEP), have been remodeled, the compressor model has been changed for a more advanced model presented in [7], the turbine has been remodeled to better describe the efficiency, and the model is validated as a complete system.

The purpose of this paper is to provide an open model to the research community, and to invoke confidence in the model by validating it. The paper is outlined as follows. In Section 1 the data sets are presented, in Section 2 the estimation criterion is presented, in Section 3 the model equations are presented and the submodels are validated, in Section 4 all submodels are connected and the complete modeled is tuned and validated against measurement data, and in Section 5 the model is simulated and its basic simulation properties are presented.

Contribution. The main contribution of this paper is a validated open-source MVEM of a Scania 13 liter 6 cylinder engine, downloadable from www.fs.isy.liu.se/Software/. Also, new component models for the engine torque, and an adaptation of an existing turbine model are introduced.

\section{Data}

Six datasets have been used for modeling and validation, see Table 1. Dataset A is dynamometer data of the engine, and is the primary modeling set. Dataset $\mathrm{B}$ is simulation data from a model of the same engine obtained using a detailed model implemented in GTPower [4]. In B, the air-to-fuel ratio is varied for the operating points and is primarily used to develop the torque model. Dataset $\mathrm{C}$ is used to model the compressor, and dataset $\mathrm{D}$ is used for modeling the turbine. Dataset $\mathrm{E}$ is used to validate the complete model, and dataset $\mathrm{F}$ is used for throttle modeling. 


\begin{tabular}{l|l|l} 
Dataset & Signals & Samples \\
\hline A. Stationary measurement data & 24 & 235 \\
B. GT Power Simulation & 22 & 160 \\
C. Compressor map & 4 & 73 \\
D. Turbine map & 4 & 38 \\
E. Dynamic measurement data & 24 & 69598 \\
F. Throttle effective area & 1 & 11 \\
\hline
\end{tabular}

Table 1: Datasets used to find model parameters.

\section{Parameter Estimation}

To parameterize the sub-models, the following criterion is used:

$$
\theta^{*}=\arg \min _{\theta} \sum_{k=1}^{K} e_{k}^{2}(\theta)
$$

where $\theta^{*}$ are the criterion minimizing parameters, $e_{k}$ the model error at sample $k$, and $K$ the number of samples in the data set.

Some of the submodels are not modeled using dataset $\mathrm{A}$, which is the desired model behavior at steady-state. To compensate for this, the parameters need to be refitted to represent dataset $\mathrm{A}$. This is done using a regularization technique, which is selected in order to preserve the model structure, according to:

$$
\theta^{\star}=\arg \min _{\theta}\left(\sum_{k=1}^{K} e_{k}^{2}(\theta)+C \sum_{i=1}^{I}\left(\mu_{i} \frac{\theta_{i}^{*}-\theta_{i}}{\theta_{i}^{*}}\right)^{2}\right)
$$

where $\theta^{\star}$ are the parameters refitted to dataset A, $\theta^{*}$ the parameters obtained using (1) and a dataset different than A, $I$ the numbers of parameters to refit, $\mu_{i}$ the weight assigned to parameter $i$, and $C$ the cost of changing the parameters.

To evaluate the model fit, the following measures are used:

$$
\begin{aligned}
& \bar{e}=\frac{1}{K} \sum_{k=1}^{K}\left|e_{k}\right| \\
& e_{\sigma}=\sqrt{V\left[\left|e_{k}\right|\right]} \\
& \bar{e}_{\text {rel }}=\frac{1}{K} \sum_{k=1}^{K}\left|\frac{e_{k}}{s_{k}}\right| \\
& e_{\text {rel }, \sigma}=\sqrt{V\left[\left|e_{k} / s_{k}\right|\right]}
\end{aligned}
$$

where $\bar{e}$ is the mean absolute error, $e_{\sigma}$ the standard deviation of the absolute error, $\bar{e}_{\text {rel }}$ the relative error, $s_{k}$ the k:th sample, and $e_{\mathrm{rel}, \sigma}$ the standard deviation of the relative error. The variance was calculated using the command var in Matlab R2017b.

\section{Model}

In the presentation of the validated model structure, the time dependence, $(t)$, is used to distinguish variables from constants, dependence on other variables is omitted for notational simplicity.

The model has four states $x(t)$ :

$$
x(t)=\left[p_{\mathrm{c}}(t), p_{\mathrm{im}}(t), p_{\mathrm{em}}(t), \omega_{\mathrm{tc}}(t)\right]
$$

where $p_{\mathrm{c}}$ is the pressure after the compressor, $p_{\text {im }}$ the intake manifold pressure, $p_{\mathrm{em}}$ the exhaust manifold pressure, and $\omega_{\mathrm{tc}}$ the turbocharger angular velocity. The model has three control inputs:

$$
u(t)=\left[u_{\mathrm{f}}(t), u_{\mathrm{thr}}(t), u_{\mathrm{wg}}(t)\right]
$$

where $u_{\mathrm{f}}$ is the fuel mass injected per cycle, $u_{\mathrm{thr}}$ the throttle position, and $u_{\mathrm{wg}}$ the wastegate position. The engine speed, $N_{\text {ice }}$, is treated as an external input into the system. Figure 1 shows an overview of the model, where the states and control inputs are visualized.

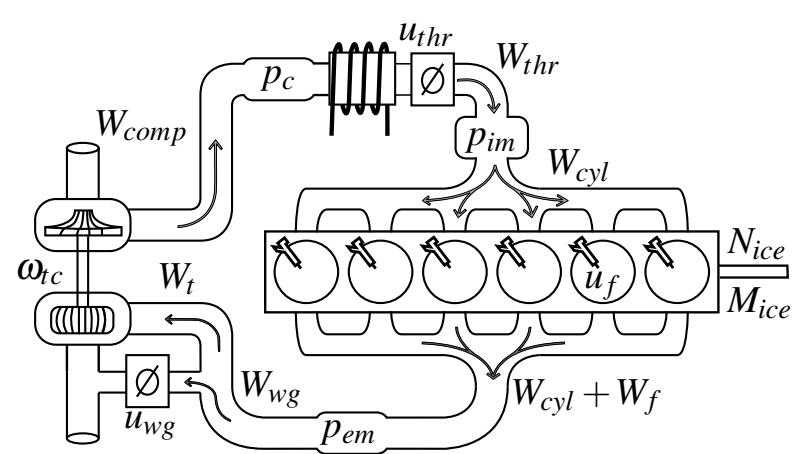

Figure 1: Model overview. Shown are the four states: pressure after the compressor $p_{\mathrm{c}}$, intake manifold pressure $p_{\text {im }}$, exhaust manifold pressure $p_{\text {em }}$ and turbocharger angular velocity $\omega_{\mathrm{tc}}$, and the three control inputs: Fuel injection per cycle $u_{\mathrm{f}}$, throttle effective area $u_{\text {thr }}$, and wastegate effective area $u_{\mathrm{wg}}$. 


\subsection{Control volumes}

The volume after the compressor, intake manifold and exhaust manifold are seen as thermodynamic control volumes, and modeled as dynamic states with filling and emptying dynamics. Using an isothermal model and by assuming mass conservation, constant $c_{\mathrm{p}}$ and $c_{\mathrm{v}}$, the dynamics are described as:

$$
\begin{aligned}
\frac{d}{d t} p_{\mathrm{c}}(t) & =\frac{R_{\mathrm{a}} T_{\mathrm{c}}(t)}{V_{\mathrm{c}}}\left(W_{c}(t)-W_{\mathrm{thr}}(t)\right) \\
\frac{d}{d t} p_{\mathrm{im}}(t) & =\frac{R_{\mathrm{a}} T_{\mathrm{im}}}{V_{\mathrm{im}}}\left(W_{\mathrm{thr}}(t)-W_{\mathrm{cyl}}(t)\right) \\
\frac{d}{d t} p_{\mathrm{em}}(t) & =\frac{R_{\mathrm{e}} T_{\mathrm{em}}(t)}{V_{\mathrm{em}}}\left(W_{\mathrm{cyl}}(t)+W_{\mathrm{f}}(t)-W_{\mathrm{t}}(t)-W_{\mathrm{wg}}(t)\right)
\end{aligned}
$$

Parameters. There are four parameters to estimate: volume after the compressor $V_{\mathrm{c}}$, intake manifold volume $V_{\mathrm{im}}$, exhaust manifold volume $V_{\mathrm{em}}$, and intake manifold temperature $T_{\mathrm{im}}$.

Parametrization and Validation. In dataset A, the mean value of the measured temperature in the intake manifold is $304.00 \mathrm{~K}$ with a standard deviation of $1.11 \mathrm{~K}$, indicating that a constant temperature in the intake manifold is an acceptable modeling assumption. The charge air cooler is assumed to be ideal, therefore the temperature in the intake manifold equals the surrounding temperature, $T_{\mathrm{im}}=T_{\mathrm{amb}}$. The sizes of the volumes $V_{\mathrm{c}}, V_{\mathrm{im}}$ and $V_{\mathrm{em}}$ are tuned until the dynamics of the model comply with the measurements. Validation of the volume sizes is seen in Section 5.

\subsection{Throttle}

In accordance with the throttle modeling approach in []MCED, the mass flow through the throttle is modeled as an isentropic compressible restriction, according to:

$$
W_{\mathrm{thr}}(t)=\frac{p_{\mathrm{c}}(t)}{\sqrt{R_{\mathrm{a}} T_{\mathrm{c}}(t)}} C_{\mathrm{D}, \mathrm{thr}} A_{\mathrm{thr}, \max } u_{\mathrm{thr}}(t) \Psi_{\mathrm{thr}}(t)
$$

where the throttle air mass flow is denoted by $W_{\text {thr }}$, the maximum throttle area by $A_{\mathrm{thr} \text { max }}$, the flow coefficient by $C_{\mathrm{D}, \text { thr }}$, the temperature by $T_{\mathrm{c}}$, and the specific gas constant of air by $R_{a}$. The throttle effective area is controlled directly via the control signal $u_{\text {thr }}(t)$. The flow parameter $\Psi_{\text {thr }}$ is taken from [9], and represented as in
[6]:

$$
\begin{gathered}
\Psi_{\mathrm{thr}}(t)=\sqrt{\frac{\gamma_{\mathrm{a}}+1}{2 \gamma_{\mathrm{a}}}\left(1-\Pi_{\mathrm{thr}}(t)\right)\left(\Pi_{\mathrm{thr}}(t)+\frac{\gamma_{\mathrm{a}}-1}{\gamma_{\mathrm{a}}+1}\right)} \\
\Pi_{\mathrm{thr}}(t)= \begin{cases}\frac{p_{\mathrm{im}}(t)}{p_{\mathrm{c}}(t)} & \text { if } \frac{p_{\mathrm{im}}(t)}{p_{\mathrm{c}}(t)} \geq \frac{1}{\gamma_{\mathrm{a}}+1} \\
\frac{1}{\gamma_{\mathrm{a}}+1} & \text { otherwise }\end{cases}
\end{gathered}
$$

where $\gamma_{a}$ is the ratio of specific heats. The conditional equation (11b) does not have a continuous derivative at the switching point. The Logistic function is therefore used to emulate the switching (11b):

$$
\begin{aligned}
\Pi_{\mathrm{thr}}(t) & =\Pi^{\text {choke }}+c_{\text {switch }}(t)\left(\Pi(t)-\Pi^{\text {choke }}\right) \\
c_{\text {switch }}(t) & =\frac{1}{1+e^{-c \Psi\left(\Pi(t)-\Pi^{\text {choke }}\right)}} \\
\Pi^{\text {choke }} & =\frac{1}{\gamma_{\mathrm{a}}+1} \\
\Pi(t) & =\frac{p_{\text {im }}(t)}{p_{\mathrm{c}}(t)}
\end{aligned}
$$

where $c \Psi$ is the steepness parameter of the Logistic function. The implemented flow parameter $\Psi_{\mathrm{thr}}(t)$ is modelled as (11a), where $\Pi_{\mathrm{thr}}(t)$ is modelled according to (12).

Parameters. There is one parameter to estimate, $C_{\mathrm{D}, \mathrm{thr}}$. The throttle area $A_{\mathrm{thr} \text { max }}$ is known from dataset $\mathrm{F}$.

Parametrization and Validation. A dataset for validating the throttle model was not obtained. $C_{\mathrm{D} \text {,thr }}$ is seen as a tuning parameter in optimizing the steadystate levels of the model. A validation of $\Psi_{\text {thr }}$ is depicted in Figure 2.

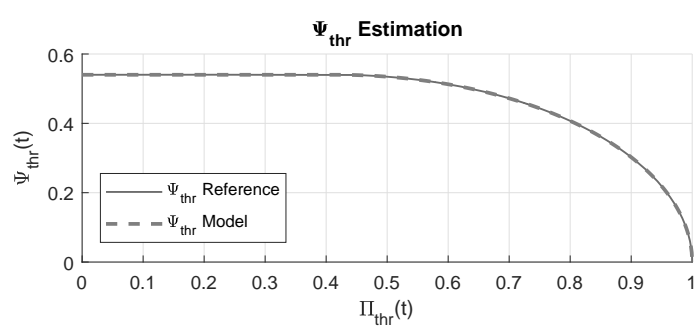

Figure 2: $\Psi_{\text {thr }}(t)$-functions where the blue solid reference is described by (11), the red trace is when $\Pi_{\mathrm{thr}}(t)$ is described according to (12). 


\subsection{Cylinder}

The cylinder air mass flow is modeled with the help of the volumetric efficiency, $\eta_{\mathrm{vol}}$, [5]. It is expressed using a single constant as in [3]:

$$
W_{\mathrm{cyl}}(t)=\eta_{\mathrm{vol}} \frac{V_{\mathrm{D}} p_{\mathrm{im}}(t)}{n_{\mathrm{r}} R_{\mathrm{a}} T_{\mathrm{im}}} \frac{N_{\mathrm{ice}}(t)}{60}
$$

The cylinder fuel mass flow is calculated from the fuel injection per cycle and engine speed:

$$
W_{\mathrm{f}}(t)=\frac{n_{\text {cyl }} N_{\text {ice }}(t) u_{\mathrm{f}}(t)}{n_{\mathrm{r}}} \frac{10^{-6}}{60}
$$

The fuel-to-air equivalence ratio $\phi(t)$ is calculated as:

$$
\phi(t)=\frac{W_{\mathrm{f}}(t)}{W_{\mathrm{cyl}}(t)} \mathrm{AF}_{\mathrm{s}}
$$

where $\mathrm{AF}_{\mathrm{S}}$ is the air-to-fuel stoichiometric ratio.

To model the engine torque $M_{\text {ice }}(t)$, it is broken down into the components: gross indicated torque $M_{\mathrm{ig}}(t)$, pumping torque $M_{\text {pump }}(t)$, and friction torque $M_{\text {fric }}(t)$, and calculated as:

$$
M_{\text {ice }}(t)=M_{\mathrm{ig}}(t)-M_{\text {pump }}(t)-M_{\text {fric }}(t)
$$

where the three components $M_{\mathrm{ig}}(t), M_{\text {pump }}(t)$ and $M_{\text {fric }}(t)$ are expressed in the normalized quantities IMEP, PMEP and FMEP [3].

The indicated torque is modeled according to:

$$
\begin{aligned}
M_{\mathrm{ig}}(t) & =\frac{V_{\mathrm{D}}}{n_{\mathrm{r}} 2 \pi} \operatorname{IMEP}(t) \\
\operatorname{IMEP}(t) & =\eta_{\mathrm{ig}}(t) \frac{q_{\mathrm{HV}} u_{\mathrm{f}}(t) 10^{-6} n_{\mathrm{cyl}}}{V_{\mathrm{D}}} \\
\eta_{\mathrm{ig}}(t) & =\left(1-\frac{1}{r_{\mathrm{c}}(t)-1}\right) \eta_{\mathrm{cal}}(t) \\
\gamma_{\mathrm{cyl}}(t) & =c_{\gamma, 0}+c_{\gamma, 1} \phi(t)+c_{\gamma, 2} \phi^{2}(t)
\end{aligned}
$$

Where the operating point dependent losses, are modeled using the following polynomial structure:

$$
\begin{aligned}
\eta_{\mathrm{cal}}(t) & =c_{\mathrm{cal}, 2}(t)\left(\frac{u_{\mathrm{f}}(t)}{100}-c_{\mathrm{cal}, 1}(t)\right)^{2}+c_{\mathrm{cal}, 0} \\
c_{\mathrm{cal}, 1}(t) & =c_{\mathrm{cal}, 10}+c_{\mathrm{cal}, 11} \frac{N_{\mathrm{ice}}(t)}{1000}
\end{aligned}
$$

$$
c_{\mathrm{cal}, 2}(t)=c_{\mathrm{cal}, 20}+c_{\mathrm{cal}, 21} \frac{N_{\mathrm{ice}}(t)}{1000}+c_{\mathrm{cal}, 22}\left(\frac{N_{\mathrm{ice}}(t)}{1000}\right)^{2}
$$

The pumping losses are modeled as:

$$
\begin{aligned}
M_{\text {pump }}(t) & =\frac{V_{\mathrm{D}}}{n_{\mathrm{r}} 2 \pi} \operatorname{PMEP}(t) \\
\operatorname{PMEP}(t) & =c_{\mathrm{PMEP}, 0}+c_{\mathrm{PMEP}, 1}\left(p_{\mathrm{em}}(t)-p_{\text {im }}(t)\right)
\end{aligned}
$$

The losses which are not included in the pumping losses are lumped into the friction term, which is modeled as a polynomial in fuel injection and engine speed:

$$
\begin{aligned}
M_{\text {fric }}(t) & =\frac{V_{\mathrm{D}}}{n_{\mathrm{r}} 2 \pi} \operatorname{FMEP}(t) \\
\operatorname{FMEP}(t) & =c_{\mathrm{f}, 0}+c_{\mathrm{f}, 1} N_{\text {ice }}(t)+c_{\mathrm{f}, 2} u_{\mathrm{f}}(t)+c_{\mathrm{f}, 3} u_{\mathrm{f}}(t) N_{\text {ice }}(t)
\end{aligned}
$$

The temperature of the gas exiting the cylinders, $T_{e}$, is modeled based on calculations on an ideal cycle and adding the parameter $\eta_{\text {sc }}$ to include non-ideal properties:

$$
\begin{aligned}
T_{\mathrm{e}}(t) & =\eta_{\mathrm{sc}} \Pi_{\mathrm{cyl}}^{1-1 / \gamma_{\mathrm{a}}}(t) r_{\mathrm{c}}^{1-\gamma_{\mathrm{a}}}\left(\frac{q_{\mathrm{in}}(t)}{c_{\mathrm{p}, \mathrm{a}}}+T_{\mathrm{im}} r_{c}^{\gamma_{\mathrm{a}}-1}\right) \\
q_{\mathrm{in}}(t) & =\frac{W_{\mathrm{f}}(t)}{W_{\mathrm{f}}(t)+W_{\mathrm{cyl}}(t)} q_{\mathrm{HV}} \\
\Pi_{\mathrm{cyl}}(t) & =\frac{p_{\mathrm{em}}(t)}{p_{\mathrm{im}}(t)}
\end{aligned}
$$

To take the heat transfer from the exhaust manifold to the surroundings into account, the mean value exhaust gas temperature model from [2] is implemented:

$$
T_{\mathrm{em}}(t)=T_{\mathrm{amb}}+\left(T_{\mathrm{e}}(t)-T_{\mathrm{amb}}\right) e^{-\frac{c_{\mathrm{em}, \mathrm{h}}}{\left(W_{\mathrm{cyl}}(t)+W_{\mathrm{f}}(t) c_{\mathrm{p}, \mathrm{e}}\right.}}
$$

Parameters. There are 18 parameters to estimate: $\eta_{\mathrm{vol}}, c_{\gamma, 0}, c_{\gamma, 1}, c_{\gamma, 2}, c_{\mathrm{cal}, 0}, c_{\mathrm{cal}, 10}, c_{\mathrm{cal}, 11}, c_{\mathrm{cal}, 20}, c_{\mathrm{cal}, 21}$, $c_{\mathrm{cal}, 22}, c_{\mathrm{f}, 0}, c_{\mathrm{f}, 1}, c_{\mathrm{f}, 2}, c_{\mathrm{f}, 3}, c_{\mathrm{PMEP}, 0}, c_{\mathrm{PMEP}, 1}, \eta_{\mathrm{sc}}$, and $c_{\mathrm{em}, \mathrm{h}}$.

Parametrization and Validation. To preserve the properties observed in the data, the parameterization is carried out in steps. The cylinder massflow is estimated using dataset A. The error function is calculated as:

$$
e_{k, \eta \mathrm{vol}}=\left(\eta_{\mathrm{vol}, \mathrm{data}, k}-\eta_{\mathrm{vol}}\right)^{2}
$$

The resulting fit is shown in Table 2. 
The indicated torque is estimated using data set B (Table 1). The parameterization was done by minimizing the following error function:

$$
\begin{aligned}
& e_{k, \mathrm{IMEP}}=\left(\operatorname{IMEP}_{\mathrm{data}, k}-\operatorname{IMEP}_{\text {model }, k}\left(\theta_{\mathrm{IMEP}}\right)\right)^{2} \\
& \theta_{\mathrm{IMEP}}=\left[c_{\gamma, 0}, c_{\gamma, 1}, c_{\gamma, 2}, c_{\mathrm{cal}, 10}, c_{\mathrm{cal}, 11}, c_{\mathrm{cal}, 20}, c_{\mathrm{cal}, 21}, c_{\mathrm{cal}, 22}\right]
\end{aligned}
$$

The resulting fit is shown in Table 3 .

The pumping torque is estimated using data set B (Table 1). The parameterization was done by minimizing the following error function:

$$
\begin{aligned}
e_{k, \mathrm{PMEP}} & =\left(\mathrm{PMEP}_{\mathrm{data}, k}-\mathrm{PMEP}_{\text {model }, k}\left(\theta_{\mathrm{PMEP}}\right)\right)^{2} \\
\theta_{\mathrm{PMEP}} & =\left[c_{\mathrm{PMEP}, 0}, c_{\mathrm{PMEP}, 1}\right]
\end{aligned}
$$

The resulting fit is shown in Table 3.

The friction torque is estimated using data set B (Table 1). The parameterization was done by minimizing the following error function:

$$
\begin{aligned}
e_{k, \mathrm{FMEP}} & =\left(\mathrm{FMEP}_{\mathrm{data}, k}-\mathrm{FMEP}_{\text {model }, k}\left(\theta_{\mathrm{FMEP}}\right)\right)^{2} \\
\theta_{\mathrm{FMEP}} & =\left[c_{\mathrm{f}, 0}, c_{\mathrm{f}, 1}, c_{\mathrm{f}, 2}, c_{\mathrm{f}, 3}\right]
\end{aligned}
$$

The resulting fit is shown in Table 3.

For the model describing the exhaust manifold temperature, the loss function is calculated as:

$$
\begin{aligned}
& e_{k, T}=\left(T_{\mathrm{em}, \text { data }, k}-T_{\mathrm{em}, \text { model }, k}\left(\theta_{T_{\mathrm{em}}}\right)\right)^{2} \\
& \theta_{T_{\mathrm{em}}}=\left[\eta_{\mathrm{sc}}, c_{\mathrm{em}, \mathrm{h}}\right]
\end{aligned}
$$

The resulting fit is shown in Table 2 .

\begin{tabular}{ccccc}
\hline & $\bar{e}$ & $e_{\sigma}$ & $\bar{e}_{\text {rel }}$ & $e_{\text {rel }, \sigma}$ \\
\hline$\eta_{\text {vol }}$ & $0.84 \%$ & $0.61 \%$ & $0.92 \%$ & $0.69 \%$ \\
$T_{\mathrm{em}}$ & $5.20 \mathrm{~K}$ & $3.40 \mathrm{~K}$ & $0.78 \%$ & $0.49 \%$ \\
\hline
\end{tabular}

Table 2: Cylinder model fit to dataset A. $\bar{e}$ is the mean absolute error, $e_{\sigma}$ the variance of the absolute error, $\bar{e}_{\text {rel }}$ the relative error, and $e_{\text {rel }, \sigma}$ the variance of the relative error.

\begin{tabular}{lcccc}
\hline & $\bar{e}$ & $e_{\sigma}$ & $\bar{e}_{\text {rel }}$ & $e_{\text {rel }, \sigma}$ \\
\hline IMEP & $24.0 \mathrm{kPa}$ & $11.7 \mathrm{kPa}$ & $1.57 \%$ & $0.42 \%$ \\
PMEP & $99.2 \mathrm{kPa}$ & $105.1 \mathrm{kPa}$ & $23.0 \%$ & $13.6 \%$ \\
FMEP & $0.75 \mathrm{kPa}$ & $0.52 \mathrm{kPa}$ & $0.70 \%$ & $0.43 \%$ \\
\hline
\end{tabular}

Table 3: Cylinder model fit to dataset B. $\bar{e}$ is the mean absolute error, $e_{\sigma}$ the variance of the absolute error, $\bar{e}_{\text {rel }}$ the relative error, and $e_{\text {rel }, \sigma}$ the variance of the relative error.

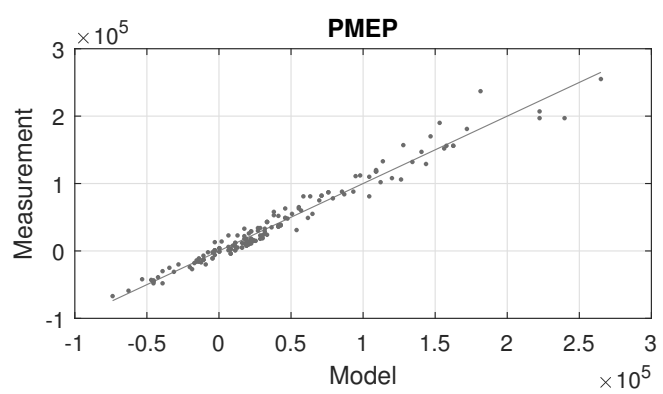

Figure 3: PMEP fit to dataset $B$. Red line shows the one-to-one ratio.

\subsection{Turbocharger}

The turbocharger dynamics is modeled from Newton's second law of motion according to:

$$
\frac{d}{d t} \omega_{\mathrm{tc}}(t)=\frac{P_{\mathrm{t}} \eta_{\mathrm{m}}(t)-P_{\mathrm{c}}(t)}{\omega_{\mathrm{tc}}(t) J_{\mathrm{tc}}}
$$

Parameters. There is one parameter to estimate: $J_{\mathrm{tc}}$. The validation is seen in Section 5.

\subsection{Turbine}

The turbine power, including the mechanical efficiency of the turbocharger shaft is calculated according to:

$$
\begin{aligned}
P_{\mathrm{t}} \eta_{\mathrm{m}}(t) & =W_{\mathrm{t}}(t) c_{\mathrm{p}, \mathrm{e}} T_{\mathrm{em}}(t) \eta_{\mathrm{t}}(t)\left(1-\Pi_{\mathrm{t}}(t)^{1-1 / \gamma_{\mathrm{e}}}\right) \\
\Pi_{\mathrm{t}}(t) & =\frac{p_{\mathrm{ats}}}{p_{\mathrm{em}}(t)}
\end{aligned}
$$

where $p_{\text {ats }}$ is the pressure in the exhaust aftertreatment system. For the flow, the square root turbine flow model in [3] is adapted to to describe dataset D:

$$
W_{\mathrm{t}, \mathrm{corr}}(t)=k_{0}(t)\left(1-\Pi_{\mathrm{t}}(t)^{k_{1}(t)}\right)^{k_{2}(t)}
$$




$$
\begin{aligned}
k_{0}(t) & =c_{00}+c_{02} N_{\mathrm{tc}, \mathrm{corr}, \mathrm{I}}^{2}(t) \\
k_{1}(t) & =c_{10}+c_{11} N_{\mathrm{tc}, \mathrm{corr}, \mathrm{I}}(t) \\
k_{2}(t) & =c_{20}+c_{21} N_{\mathrm{tc}, \mathrm{corr}, \mathrm{I}}(t)+c_{22} N_{\mathrm{tc}, \mathrm{corr}, \mathrm{I}}^{2}(t) \\
N_{\mathrm{tc}, \mathrm{corr}, \mathrm{I}}(t) & =\frac{\omega_{\mathrm{tc}}(t)}{\sqrt{T_{e m}(t)}} \frac{30}{\pi} \frac{1}{1000}
\end{aligned}
$$

The turbine mass flow is calculated as:

$$
W_{\mathrm{t}}(t)=W_{\mathrm{t}, \mathrm{corr}}(t) \frac{p_{\mathrm{em}} 10^{-3}}{\sqrt{T_{\mathrm{em}}}}
$$

For the turbine efficiency, the standard approach of modeling it from the blade-speed-ratio (BSR) is taken. However, since the BSR lines do not overlap in the $\mathrm{BSR}-\eta$ plane in dataset $\mathrm{D}$, a speed dependence is included. The model is described by:

$$
\begin{aligned}
& \eta_{t}(t)=\eta_{t, \max }(t)-k_{\eta}(t)\left(\mathrm{BSR}(t)-\mathrm{BSR}_{\mathrm{opt}}(t)\right)^{2} \\
& \operatorname{BSR}(t)= \frac{\omega_{\mathrm{tc}}(t) D_{\mathrm{t}} / 2}{\sqrt{2 c_{\mathrm{p}, \mathrm{e}} T_{\mathrm{em}}(t)\left(1-\Pi_{\mathrm{t}}(t){ }^{1-\frac{1}{\gamma_{\mathrm{e}}}}\right)}} \\
& \operatorname{BSR}_{\mathrm{opt}}(t)= c_{\mathrm{BSR}, 0}+c_{\mathrm{BSR}, 1} N_{\mathrm{tc}, \mathrm{corr}, \mathrm{II}}(t)+ \\
& \eta_{\mathrm{t}, \mathrm{max}}(t) c_{\mathrm{BSR}, 2} N_{\mathrm{tc}, \mathrm{corr}, \mathrm{II}}(t) \\
& k_{\eta}(t)= c_{\eta_{t}, 0}+c_{\eta_{t}, 1} N_{\mathrm{tc}, \mathrm{corr}, \mathrm{II}}+(t) \\
& N_{\mathrm{tc}, \mathrm{corr}, \mathrm{II}}(t)=\frac{\omega_{\mathrm{tc}}(t)}{\sqrt{T_{e m}(t)} \frac{30}{\pi} \frac{1}{10000} N_{\mathrm{tc}, \mathrm{corr}, \mathrm{II}}(t)}
\end{aligned}
$$

Parameters. There are 14 parameters to estimate: $c_{00}, c_{02}, c_{10}, c_{11}, c_{20}, c_{21}, c_{22}, c_{\mathrm{BSR}, 0}, c_{\mathrm{BSR}, 1}, c_{\mathrm{BSR}, 2}$, $c_{\eta_{t}, 0}, c_{\eta_{t}, 1}, c_{\max , 0}$, and $c_{\max , 1}$.

Parametrization and Validation. The parameterization is carried out by parameterizing the mass flow model and efficiency model separately using dataset D. For the mass flow, the loss function is calculated as:

$$
\begin{aligned}
& e_{k, W \mathrm{t}}=\left(W_{\mathrm{t}, \text { data, } k}-W_{\mathrm{t}, \text { model }, k}\left(\theta_{W \mathrm{t}}\right)\right)^{2}
\end{aligned}
$$

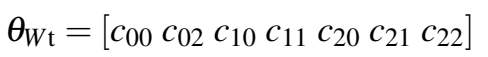

The model fit is shown in Table 4. For the efficiency, the following loss function is used:

$$
e_{k, \eta \mathrm{t}}=\left(\eta_{\mathrm{t}, \text { data }, k}-\eta_{\mathrm{t}, \text { model }, k}\left(\theta_{\eta \mathrm{t}}\right)\right)^{2}
$$

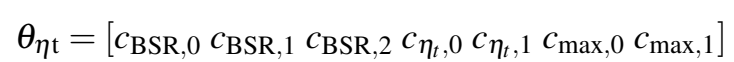

The model fit is shown in Table 4.

\subsection{Wastegate}

The wastegate is developed in the same way as the throttle (see section 3.2), apart from $\gamma_{\mathrm{a}}$ which is replaced by $\gamma_{\mathrm{e}}$. The wastegate mass flow is described by:

$$
W_{\mathrm{wg}}(t)=\frac{p_{\mathrm{em}}(t)}{\sqrt{R_{\mathrm{e}} T_{\mathrm{em}}}} C_{\mathrm{D}, \mathrm{wg}} A_{\mathrm{wg}, \max } u_{\mathrm{wg}}(t) \Psi_{\mathrm{wg}}(t)
$$

where $\Psi_{\mathrm{wg}}(t)$ is similar to (11a), but $\Pi_{\mathrm{thr}}(t)$ is replaced by $\Pi_{\mathrm{wg}}(t) . \Pi_{\mathrm{wg}}(t)$ is defined as in Equation (12), where $\Pi(t)$ is replaced by $\Pi_{t}(t)$ in $(29 b)$.

Parameters There is only one parameter to estimate, $C_{\mathrm{D}, \mathrm{wg}}$. The wastegate area $A_{\mathrm{wg}, \max }$ is determined from measuring the diameter. $C_{\mathrm{D}, \mathrm{wg}}$ is seen as a tuning constant. Since data was not available to parameterize the wastegate as a separate component, the same approach as for the throttle is taken. The modeling of the wastegate and throttle is similar due to both being controllable valves for restricting the gas flow.

\subsection{Compressor}

The parameterization of the compressor was done using dataset $\mathrm{C}$ and by using LiU CPgui [8], which parameterizes a high-order control-oriented compressor model based on the total least squares algorithm. The fit to dataset A is shown in Table 5.

\section{Full System Parametrization}

An approach taken in [11] and [10] is to refit the parameters to the measurements when all model components

\begin{tabular}{ccccc}
\hline & $\bar{e}$ & $e_{\sigma}$ & $\bar{e}_{\text {rel }}$ & $e_{\text {rel }, \sigma}$ \\
\hline$W_{\mathrm{t}, \mathrm{corr}}$ & $5.1410^{-4}$ & $3.5610^{-4}$ & $1.30 \%$ & $0.95 \%$ \\
& $\mathrm{~kg} / \mathrm{s} \sqrt{\mathrm{K}} / \mathrm{kPa}$ & $\mathrm{kg} / \mathrm{s} \sqrt{\mathrm{K}} / \mathrm{kPa}$ & & \\
$\eta_{\mathrm{t}}$ & $0.92 \%$ & $0.99 \%$ & $1.34 \%$ & $1.47 \%$ \\
\hline
\end{tabular}

Table 4: Turbine model fit to dataset D. $\bar{e}$ is the mean absolute error, $e_{\sigma}$ the variance of the absolute error, $\bar{e}_{\text {rel }}$ the relative error, and $e_{\text {rel }, \sigma}$ the variance of the relative error. 


\begin{tabular}{ccccc}
\hline & $\bar{e}$ & $e_{\sigma}$ & $\bar{e}_{\text {rel }}$ & $e_{\text {rel }, \sigma}$ \\
\hline$W_{\mathrm{c}}$ & $1.0310^{-4}$ & $8.2910^{-4}$ & $0.13 \%$ & $1.51 \%$ \\
& $\mathrm{~kg} / \mathrm{s}$ & $\mathrm{kg} / \mathrm{s}$ & & \\
$\eta_{\mathrm{c}}$ & $0.92 \%$ & $0.99 \%$ & $1.34 \%$ & $1.47 \%$ \\
\hline
\end{tabular}

Table 5: Compressor model fit to dataset A. $\bar{e}$ is the mean absolute error, $e_{\sigma}$ the variance of the absolute error, $\bar{e}_{\text {rel }}$ the relative error, and $e_{\text {rel }, \sigma}$ the variance of the relative error.

are assembled in order to achieve good fit. A similar approach is taken here, but with a slightly different cost function (see equation 2) where a regularization technique is used to limit the changes in parameter values, in order to preserve the model structure. This procedure is divided into two steps. The first step is to refit the torque model to dataset $\mathrm{A}$. The second step is to tune all parameters influencing the steady state levels of the states when the complete model is fully assembled.

\subsection{Torque model}

For the torque model, the following parameters are refitted:

$$
\begin{array}{r}
\theta_{\mathrm{M}}=\left[c_{\mathrm{cal}, 0}, c_{\mathrm{cal}, 10}, c_{\mathrm{cal}, 11}, c_{\mathrm{cal}, 20}, c_{\mathrm{cal}, 21}, c_{\mathrm{cal}, 22},\right. \\
\left.c_{\mathrm{f}, 0}, c_{\mathrm{f}, 1}, c_{\mathrm{f}, 2}, c_{\mathrm{f}, 3}, c_{\mathrm{PMEP}, 0}, c_{\mathrm{PMEP}, 1}\right]
\end{array}
$$

using criterion (2). The resulting fit is shown in Table 6 .

\begin{tabular}{ccccc}
\hline & $\bar{e}$ & $e_{\sigma}$ & $\bar{e}_{\text {rel }}$ & $e_{\text {rel }, \sigma}$ \\
\hline$M_{\text {ice }}$ & $8.42 \mathrm{Nm}$ & $6.59 \mathrm{Nm}$ & $1.81 \%$ & $3.05 \%$ \\
\hline
\end{tabular}

Table 6: Torque model refit to dataset A. $\bar{e}$ is the mean absolute error, $e_{\sigma}$ the variance of the absolute error, $\bar{e}_{\text {rel }}$ the relative error, and $e_{\text {rel }, \sigma}$ the variance of the relative error.

\subsection{Tuning of steady-state levels}

In the same manner as for the torque model, using criterion (2), the parameters influencing the steady-state levels of the states are re-parameterized. To do this, the full model is simulated and the wastegate is controlled, using a $P I D$-controller to minimize the error:

$$
e_{\mathrm{wg}}=W_{\mathrm{c}}-W_{\mathrm{c}, \mathrm{ref}}
$$

where the compressor massflow reference $W_{\mathrm{c}, \text { ref }}$ is taken from the massflow measurment in dataset $\mathrm{A}$. The result- ing fit is shown in Table 7.

\begin{tabular}{ccccc}
\hline & $\bar{e}$ & $e_{\sigma}$ & $\bar{e}_{\text {rel }}$ & $e_{\text {rel }, \sigma}$ \\
\hline$p_{\mathrm{c}}$ & $2.78 \mathrm{kPa}$ & $4.00 \mathrm{kPa}$ & $1.89 \%$ & $2.80 \%$ \\
$p_{\mathrm{im}}$ & $2.76 \mathrm{kPa}$ & $3.96 \mathrm{kPa}$ & $1.89 \%$ & $2.79 \%$ \\
$p_{\mathrm{em}}$ & $14.73 \mathrm{kPa}$ & $3.96 \mathrm{kPa}$ & $7.33 \%$ & $3.69 \%$ \\
$\omega_{\mathrm{tc}}$ & $1.98 \mathrm{kRPM}$ & $2.42 \mathrm{kRPM}$ & $4.88 \%$ & $7.93 \%$ \\
\hline
\end{tabular}

Table 7: Steady-state levels of states fit to dataset A for the fully parameterized model. $\bar{e}$ is the mean absolute error, $e_{\sigma}$ the variance of the absolute error, $\bar{e}_{\text {rel }}$ the relative error, and $e_{\mathrm{rel}, \sigma}$ the variance of the relative error.

\section{Full System Validation}

The complete system model is simulated with the control signals recorded from a dynamic engine test (part of data set E). The result from the simulated system, in comparison with the measurement data is displayed in Figure 4.

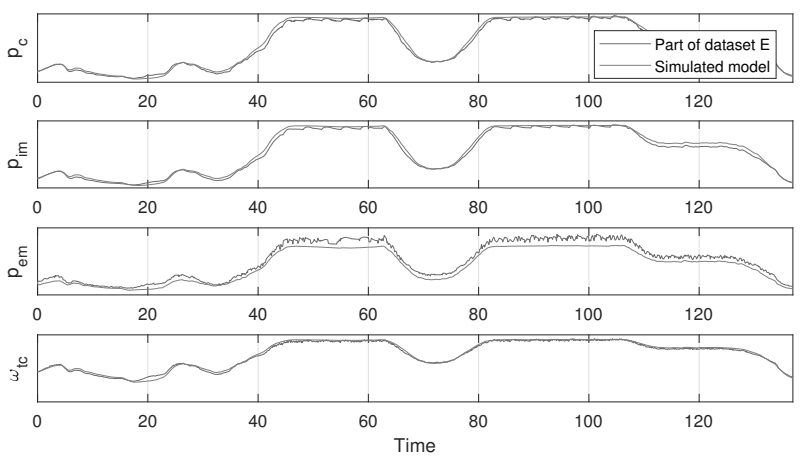

Figure 4: State validation, simulated engine model compared to dynamic measurement data. The control signals are taken from measurements.

\section{Conclusions}

A mean value engine model of a Scania 13 liters heavyduty diesel engine has been developed and validated using both stationary and dynamic measurements. The results show good agreement with measurements, showing that both dynamics and steady-state levels are well represented indicating that the model is well suited for studying the engine dynamics and fuel optimal control. The model is open-source and downloadable from www.fs.isy.liu.se/Software/. 


\section{References}

[1] Ekberg K, Leek V, Eriksson L. Optimal control of wastegate throttle and fuel injection for a heavy-duty turbocharged diesel engine during tip-in. In 58th Conference on Simulation and Modelling (SIMS 58). Reykjavik, Iceland, 2017.

[2] Eriksson L. Mean value models for exhaust system temperatures. SAE 2002 World Congress \& Exhibition, 2002.

[3] Eriksson L, Nielsen L. Modeling and Control of Engines and Drivelines. John Wiley and Sons Ltd, 2014.

[4] Gamma Technologies. GT-Power User's Manual. GTSuite Version 6.1, 2004.

[5] Heywood JB. Internal Combustion Engine Fundamentals. McGraw-Hill, 1988.

[6] Holmbom R, Eriksson L. Analysis and development of compact models for mass flows through butterfly throttle valves. In WCX World Congress Experience. SAE International, Apr 2018.
[7] Llamas X, Eriksson L. Control-oriented compressor model with adiabatic efficiency extrapolation. $S A E$ International Journal of Engines, 10(4), 2017.

[8] Llamas X, Eriksson L. LiU CPgui: A toolbox for parameterizing compressor models. Technical Report LiTH-ISY-R-3102, Department of Electrical Engineering, Linköpings Universitet, SE-581 83 Linköping, Sweden, 2018.

[9] Shen T, Ohata A. Modeling and Control Design for Automotive Engines - with MATLAB Engine Simulator CD-ROM. ISBN 978e-4-339-04610-6, 2011.

[10] Sivertsson M, Eriksson L. Modeling for Optimal Control: A Validated Diesel-Electric Powertrain Model. SIMS 2014 - 55th International Conference on Simulation and Modelling, Ålborg, Denmark, 2011.

[11] Wahlström J, Eriksson L. Modelling diesel engines with a variable-geometry turbocharger and exhaust gas recirculation by optimization of model parameters for capturing non-linear system dynamics. Proceedings of the Institution of Mechanical Engineers, Part D: Journal of Automobile Engineering, 225(7):960-986, 2011. 


\title{
Time- and Event-oriented Spreadsheet Modelling of ARGESIM Benchmark C12 'Collision Processes in Rows of Spheres'
}

\author{
Zsofia Kolozsvari ${ }^{1}$, Lukas Lienbacher ${ }^{1}$, Stefan Perebner ${ }^{1}$, Henrique Teixeira Rego ${ }^{1}$, \\ Martin Bicher ${ }^{2 *}$, Andreas Körner ${ }^{1}$, Horst Ecker ${ }^{3}$, Felix Breitenecker ${ }^{1}$ \\ ${ }^{1}$ Mathematical Modelling and Simulation Group, Inst. of Analysis and Scientific Computing, \\ 2Inst. of Information System Engineering, ${ }^{3}$ Inst. of Mechanics and Mechatronics \\ TU Wien, Wiedner Hauptstrasse 8-10, 1040 Vienna, Austria; *martin.bicher@tuwien.ac.at
}

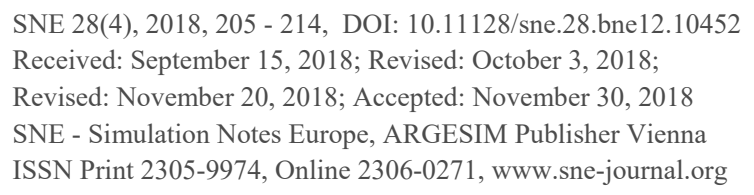

SNE 28(4), 2018, 205 - 214, DOI: 10.11128/sne.28.bne12.10452 Received: September 15, 2018; Revised: October 3, 2018;

Revised: November 20, 2018; Accepted: November 30, 2018 SNE - Simulation Notes Europe, ARGESIM Publisher Vienna ISSN Print 2305-9974, Online 2306-0271, www.sne-journal.org

Abstract. This Benchmark Study with educational key aspects presents a spreadsheet-based approach to ARGESIM Benchmark C12 'Collision Processes in Rows of Spheres'. The process, the collision of spheres in a row, is seen as discrete process with a discrete time base, using two modelling approaches. A classical time-oriented approach describes the movement of the spheres by a discrete-time model, the collisions are approximated within the discretization points. The event-oriented approach makes use of the explicit movement formula and determines a sequence of exact collision times and collision events. Both approaches are implemented in the spreadsheet program Excel, which is quite suitable for simulation of discrete processes by means of recursive formulas. The implementation uses standard features of Excel, so that the provided sources can be used in arbitrary spreadsheet programs. Interestingly, with given medium accuracy and sufficient time resolution, time-oriented and event-oriented results coincide. The study also concentrates on educational aspects in giving a sketch on the physical background of elastic and inelastic collisions, in giving hints for proper implementation, and in providing background information on the selection of required experiments with the model (benchmark tasks).

\section{Introduction}

The ARGESIM Benchmark C12 Collision Processes in Rows of Spheres, defined in SNE in 1999 ([1]), is based on a continuous mechanical model with collision events, but mainly concentrates on discrete events within the movement of the spheres - on the collision of the spheres and tries to analyse the phenomena of collisions, from elastic to plastic, or inelastic, resp. The tasks of the benchmark the experiments to be performed with the model - require determination of collision sequences depending on the collision type (between elastic and inelastic), boundary value problems for initial hits, analysis of the number of collisions and of final velocities of the spheres, and stochastic analysis for stochastically modelled collisions strength.

A time-oriented and an event-oriented discrete model approach is implemented in the spreadsheet program Excel, which is quite suitable for simulation of discrete processes by means of recursive formulas (in case of time constants of medium or large range). The implementation uses standard features of the spreadsheet tool Excel, so that the provided sources can be used in arbitrary spreadsheet programs.

Section 1 sketches the model and necessary equations, formulas, and algorithms for movement and for collisions. Section 2 presents two discrete model approaches - time-oriented and event-oriented - and the respective implementation in Excel. Section 3 presents results of time domain analysis for specific collision types in a comparative manner for the different implementation approaches. Section 4 shows the results for a structural collision analysis investigating the dependence of the number of collisions and of the final velocities of the spheres on the collisions coefficient. Section 5 sketches the experiment description and the results for a boundary value problem for the initial velocity, and the stochastic analysis of collision strength with results for final velocities.

\section{Model Equations}

The model consists of four spheres in a row, with positions $x_{i}$, velocities $v_{i},\left(\dot{x}_{i}\right)$ and parameters mass $m_{i}$, diameter $d$ and distance $a$ (Figure 1).

The first sphere starts moving, hits the second, both move, the second hits the third, the first may re-hit the second, the third hits the fourth sphere, etc. 


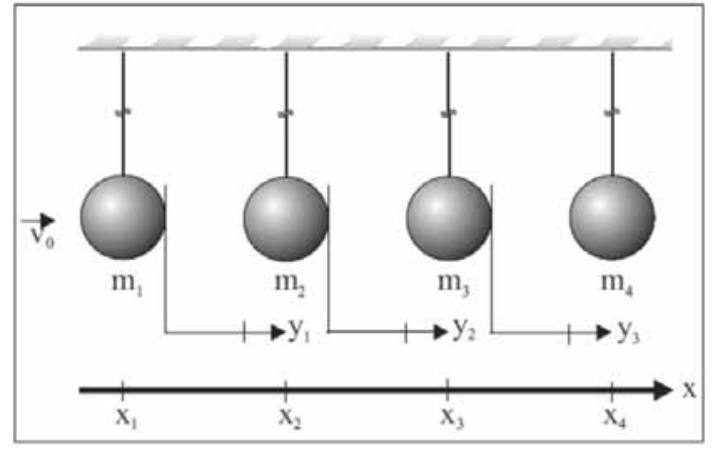

Figure 1: Spheres in a row - pendulum with infinite length.

With development of time, a sequence of collisions occurs - from minimal three collisions up to a theoretically infinite number of collisions $n_{C}$. Time instants of collisions are denoted by $t_{c}^{a}$ or $t_{c, k}^{a}$ resp. Movements of the spheres after each collision follow a simple linear translational movement, i.e.

$$
\begin{gathered}
x_{i}(t)=v_{i, c} \cdot t+x_{i, c}, \quad \mathrm{i}=1, \ldots, 4 \leftrightarrow \\
\ddot{x}_{i}(t)=0, \dot{x}_{i}\left(t_{c}^{a}\right)=v_{i, c}, \quad x_{i}(0)=x_{i, c}
\end{gathered}
$$

Here $x_{i, c}$ and $v_{i, c}=\dot{x}_{i, c}$ denote initial position and initial velocity after a collision: position is continued, and velocity is changed if the $i$-th sphere is involved, otherwise continued.

The effect of the collision is given by the momentum conservation law for the impact of two masses (Figure 2): the quality of impact is controlled by the collisions coefficient $e, 0 \leq e \leq 1$, or restitution coefficient resp., and may range from elastic collision (collisions coefficient $e_{e l}=1$ ), via the quasi-elastic collision (all spheres move with same velocity), until the inelastic or plastic collision (collisions coefficient $e_{p l}=0$ ).

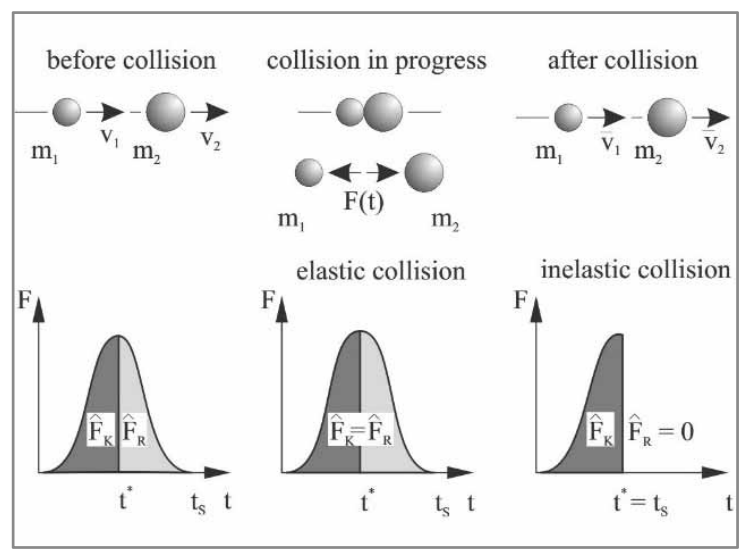

Figure 2: Central impact of two masses - momentum conservation law.
The change of velocities in case of collision at $t_{c}^{a}$ is based on the momentum conservation law depending on the collision (restitution) coefficient (Figure 2):

$$
\begin{aligned}
v_{i, \text { new }} & =v_{i}(1-e) \frac{m_{i+1}}{m_{i}+m_{i+1}}\left(v_{i}-v_{i+1}\right) \\
v_{i+1, \text { new }} & =v_{i+1}(1+e) \frac{m_{i}}{m_{i}+m_{i+1}}\left(v_{i}-v_{i+1}\right)
\end{aligned}
$$

In order to avoid small faulty differences, relative quantities $y_{j}=x_{j+1}-x_{j}-d, j=1, \ldots, 3, \dot{y}_{j}=\dot{x}_{j+1}-\dot{x}_{j}=$ $v_{j+1}-v_{j}$ have to be used as further variables.

The process starts with an active hit of the first sphere with an initial velocity $v_{0}$ :

$$
v_{1, c}=\dot{x}_{1, c}=v_{0} \text { at } t_{c, 0}^{a}=t_{0}=0 \text {, with } x_{1, c}=0
$$

resulting in initial values for the other variables with

$$
y_{1, c}=y_{2, c}=y_{3, c}=a ; \dot{y}_{1, c}=-v_{0}, \dot{y}_{2, c}=\dot{y}_{3, c}=0
$$

so that next movement starting at collision time $t_{c}^{a}$ is given by

$$
\begin{gathered}
\ddot{x}_{1}=0, \quad \dot{x}_{1}\left(t_{c}^{a}\right)=\dot{x}_{1, c}, \quad x_{1}\left(t_{c}^{a}\right)=x_{1, c} \\
\ddot{y}_{j}=0, \quad \dot{y}_{j}\left(t_{c}^{a}\right)=\dot{y}_{j, c}, \quad y_{j}\left(t_{c}^{a}\right)=y_{j, c}, j=1, \ldots 3
\end{gathered}
$$

The change of velocities at collision $C_{2 \times 3}$ (collision second sphere -third sphere) at collision time $t_{c}^{a}$ is given by momentum law (2):

$$
\begin{gathered}
\dot{y}_{1}=\dot{y}_{1}+\frac{(1+e) * m_{3}}{m_{3}+m_{2}} * \dot{y}_{2}, \\
\dot{y}_{3}=\dot{y}_{3}+\frac{(1+e) * m_{2}}{m_{3}+m_{2}} * \dot{y}_{2}, \quad \dot{y}_{2}=-e * \dot{y}_{2}
\end{gathered}
$$

These formulas for the collision $C_{u \times(u+1)}, 2 \leq u \leq n-1$ of two inner spheres is generally valid, also in case of $n$ spheres $n>4$. Similar formulas hold for collisions with first sphere $C_{1 \times 2}$ and last sphere $C_{3 \times 4} C_{(n-1) \times n}$ :

$$
\begin{gathered}
\dot{x}_{1}=\dot{x}_{1}+\frac{(1+e) * m_{2}}{m_{1}+m_{2}} * \dot{y}_{1}, \dot{y}_{1}=-e * \dot{y}_{1} \\
\dot{y}_{2}=\dot{y}_{2}+\frac{(1+e) * m_{1}}{m_{1}+m_{2}} * \dot{y}_{1} \\
\dot{y}_{2}=\dot{y}_{2}+\frac{(1+e) * m_{4}}{m_{3}+m_{4}} * \dot{y}_{3}, \dot{y}_{3}=-e * \dot{y}_{3}
\end{gathered}
$$

The simple linear translational movement (1) allows to calculate the collision times in advance. After collision time $t_{c, k}^{a}$, two neighbouring spheres move with

$$
x_{i}(t)=v_{i, c} \cdot t+x_{i, c}, \quad x_{i+1}(t)=v_{i+1, c} \cdot t+x_{i+1, c}
$$

and collide if $v_{i+1, c}<v_{i, c}$ and $x_{i+1}(t)=x_{i}(t)$, given by

$$
v_{i, c} \cdot t+x_{i, c}=v_{i+1, \mathrm{c}} \cdot t+x_{i+1, \mathrm{c}},
$$

This results in the relative timespan $t_{c, i \times i+1}^{r}$ until next collision $C_{i \times(i+1)}$ : 


$$
t=t_{c, i \times i+1}^{r}=\frac{x_{i+1, c}-x_{i, c}}{v_{i, c}-v_{i+1, c}}=\frac{y_{i, c}}{-\dot{y}_{i, c}}
$$

Depending on the velocity differences, one, two, three, or no collision can occur, where one must be the first. Consequently, the absolute time instant $t_{c, k+1}^{a}$ is given by

$$
t_{c, k+1}^{a}=t_{c, k}^{a}+\min _{\exists i \times i+1}\left(t_{c, i \times i+1}^{r}\right)
$$

Obviously, for the dynamics of movement more complicated dynamics can be used, e.g. taking into account air resistance, so that instead of linear translational movement (1) nonlinear dynamics must be used:

$$
\dot{x}_{i}(t)=v_{i}(\mathrm{t}), \dot{v}_{i}(\mathrm{t})=f\left(x_{i}, v_{i}\right), \mathrm{i}=1, \ldots, 4
$$

\section{Implementation}

Two types of approaches are implemented in MS Excel: a time-oriented approach and an event-oriented approach. The event-oriented approach computes the exact collision times, whereas the time-oriented approach detects the collisions within discrete time progress.

\subsection{Time-oriented approach}

The time-oriented approach defines a time grid with given step size $\Delta \mathrm{t}$ and calculates the dynamics in a recursive manner by a discretisation of model (1)

$$
x_{i}\left(t_{k+1}\right)=v_{i, c} \cdot \Delta \mathrm{t}+x_{i}\left(t_{k}\right), \mathrm{i}=1, \ldots, 4,
$$

and analogously for the derivative variables and difference variables according to model (3-4).
In each recursive calculation (9) the occurrence of a collision is checked. If a collision has happened in the last recursion (between $t_{k}$ and $t_{k+1}$ ) then it is handled by means of formulas (5) and (6) at time instant $t_{k+1}-$ in general too late, but proper choice of the timestep can keep the error small.

The straightforward implementation in Excel makes use of the following variables, calculated in rows and partly updated recursively along the time column:

$\mathbf{t}$ current time $t_{k}$

$\begin{array}{lllll}\mathbf{x} 1 & \mathbf{x} 2 & \mathbf{x} 3 & \mathbf{x} 4 & x_{i}\left(t_{k}\right) \text { position of spheres }\end{array}$

dx1 dx2 dx3 dx4 $\dot{x}_{i}\left(t_{k}\right)$ velocity of spheres

y1 $\mathbf{y 2}$ y3 $y_{i}\left(t_{k}\right)$ position differences

dy1 dy2 dy3 $\quad \dot{y}_{i}\left(t_{k}\right)$ velocity differences

s12 s23 s34 collision indicator at $\left(t_{k}\right)($ ' 0 ' or ' 1 ')

delta_y1 delta_y2 delta_y3 $y_{i}\left(t_{k}\right)-y_{i}\left(t_{k-1}\right)$

recursive difference of position differences.

Parameters are defined in fixed calls and named:

\section{a, d, v_0, m_1, m_2, m_3, m_4, e, t_0, delta_t, tend.}

Figure 3 shows the Excel worksheet with parameter definitions and results of the recursive updates, to be explained in more detail in the following.

The implementation of the model and its formulas is straightforward and briefly sketched in Figure 3 for row

\begin{tabular}{|c|c|c|c|c|c|c|c|c|c|c|c|c|c|c|c|c|c|c|c|c|c|c|c|}
\hline & A & B & D & $\mathrm{E}$ & $\mathrm{F}$ & G & $\mathrm{H}$ & 1 & $J$ & $\mathrm{~K}$ & L & M & $\mathrm{N}$ & 0 & $\mathrm{P}$ & Q & $\mathrm{R}$ & $\leqslant U$ & $\mathrm{v}$ & w & $\mathrm{x}$ & $\mathrm{Y}$ & Z \\
\hline 1 & ARGESIM E & BENCH & HMARK CI & 2 'Collit: & on of $\mathrm{S}$ & Seres' & ime-or & ented $A$ & oproacl & & & & & & & & & & & & & & \\
\hline 2 & & & & & & & & & & & & & & & & & & \multicolumn{3}{|c|}{ Impact } & \multirow{2}{*}{\multicolumn{3}{|c|}{$\begin{array}{l}\text { Difference: Previous Pos. Diff. } \\
\text { minus Actual position Diff. }\end{array}$}} \\
\hline 3 & \multicolumn{2}{|l|}{ Parameters } & Time & \multicolumn{4}{|c|}{ Position Variables } & \multicolumn{4}{|c|}{ Velocity Variables } & \multicolumn{3}{|c|}{ Position Difference Var. } & \multicolumn{3}{|c|}{ Velocity Difference Var. } & \multicolumn{3}{|c|}{ Indicator } & & & \\
\hline 4 & & & $\mathrm{t}$ & $\mathrm{x} 1$ & $\times 2$ & $x_{3}$ & $\times 4$ & $\mathrm{dx} 1$ & $\mathrm{~d} \times 2$ & $\mathrm{~d} \times 3$ & $d \times 4$ & $\mathrm{y} 1$ & $\mathrm{y}^{2}$ & $\mathrm{y}^{3}$ & dy1 & dy2 & dy3 & s12 & $523 \mathrm{~s}$ & s34 & delta_y1 & delta y2 & delta y3 \\
\hline 5 & a & 1 & 0,00 & 0,00 & 2,00 & 4,00 & 6,00 & 1,00 & 0,00 & 0,00 & 0,00 & 1,00 & 1,00 & 1,00 & $-1,00$ & 0,00 & 0,00 & & & & & & \\
\hline 6 & d & 1 & 0,05 & 0,05 & 2,00 & 4,00 & 6,00 & 1,00 & 0,00 & 0,00 & 0,00 & 0,95 & 1,00 & 1,00 & $-1,00$ & 0,00 & 0,00 & 0 & 0 & 0 & 0,05 & 0,00 & 0,00 \\
\hline 7 & v_o & 1 & 0,10 & 0,10 & 2,00 & 4,00 & 6,00 & 1,00 & 0,00 & 0,00 & 0,00 & 0,90 & 1,00 & 1,00 & $-1,00$ & 0,00 & 0,00 & 0 & 0 & 0 & 0,05 & 0,00 & 0,00 \\
\hline 8 & m_1 & 1 & 0,15 & 0,15 & 2,00 & 4,00 & 6,00 & 1,00 & 0,00 & 0,00 & 0,00 & 0,85 & 1,00 & 1,00 & $-1,00$ & 0,00 & 0,00 & 0 & 0 & 0 & 0,05 & 0,00 & 0,00 \\
\hline 9 & m_2 & 1 & 0,20 & 0,20 & 2,00 & 4,00 & 6,00 & 1,00 & 0,00 & 0,00 & 0,00 & 0,80 & 1,00 & 1,00 & $-1,00$ & 0,00 & 0,00 & 0 & 0 & 0 & 0,05 & 0,00 & 0,00 \\
\hline 10 & m_3 & 1 & 0,25 & 0,25 & 2,00 & 4,00 & 6,00 & 1,00 & 0,00 & 0,00 & 0,00 & 0,75 & 1,00 & 1,00 & $-1,00$ & 0,00 & 0,00 & 0 & 0 & 0 & 0,05 & 0,00 & 0,00 \\
\hline 11 & m_4 & 1 & 0,30 & 0,30 & 2,00 & 4,00 & 6,00 & 1,00 & 0,00 & 0,00 & 0,00 & 0,70 & 1,00 & 1,00 & $-1,00$ & 0,00 & 0,00 & 0 & 0 & 0 & 0,05 & 0,00 & 0,00 \\
\hline 12 & e & 0,2 & 0,35 & 0,35 & 2,00 & 4,00 & 6,00 & 1,00 & 0,00 & 0,00 & 0,00 & 0,65 & 1,00 & 1,00 & $-1,00$ & 0,00 & 0,00 & 0 & 0 & 0 & 0,05 & 0,00 & 0,00 \\
\hline 13 & to & 0 & 0,40 & 0,40 & 2,00 & 4,00 & 6,00 & 1,00 & 0,00 & 0,00 & 0,00 & 0,60 & 1,00 & 1,00 & $-1,00$ & 0,00 & 0,00 & 0 & 0 & 0 & 0,05 & 0,00 & 0,00 \\
\hline 14 & delta_t & 0,05 & 0,45 & 0,45 & 2,00 & 4,00 & 6,00 & 1,00 & 0,00 & 0,00 & 0,00 & 0,55 & 1,00 & 1,00 & $-1,00$ & 0,00 & 0,00 & 0 & 0 & 0 & 0,05 & 0,00 & 0,00 \\
\hline 15 & tend & 15 & 0,50 & 0,50 & 2,00 & 4,00 & 6,00 & 1,00 & 0,00 & 0,00 & 0,00 & 0,50 & 1,00 & 1,00 & $-1,00$ & 0,00 & 0,00 & 0 & 0 & 0 & 0,05 & 0,00 & 0,00 \\
\hline 16 & & & 0,55 & 0,55 & 2,00 & 4,00 & 6,00 & 1,00 & 0,00 & 0,00 & 0,00 & 0,45 & 1,00 & 1,00 & $-1,00$ & 0,00 & 0,00 & 0 & 0 & 0 & 0,05 & 0,00 & 0,00 \\
\hline 17 & & & 0,60 & 0,60 & 2,00 & 4,00 & 6,00 & 1,00 & 0,00 & 0,00 & 0,00 & 0,40 & 1,00 & 1,00 & $-1,00$ & 0,00 & 0,00 & 0 & 0 & 0 & 0,05 & 0,00 & 0,00 \\
\hline 18 & & & 0,65 & 0,65 & 2,00 & 4,00 & 6,00 & 1,00 & 0,00 & 0,00 & 0,00 & 0,35 & 1,00 & 1,00 & $-1,00$ & 0,00 & 0,00 & 0 & 0 & 0 & 0,05 & 0,00 & 0,00 \\
\hline 19 & Number & 13 & 0,70 & 0,70 & 2,00 & 4,00 & 6,00 & 1,00 & 0,00 & 0,00 & 0,00 & 0,30 & 1,00 & 1,00 & $-1,00$ & 0,00 & 0,00 & 0 & 0 & 0 & 0,05 & 0,00 & 0,00 \\
\hline 20 & Collisions & & 0,75 & 0,75 & 2,00 & 4,00 & 6,00 & 1,00 & 0,00 & 0,00 & 0,00 & 0,25 & 1,00 & 1,00 & $-1,00$ & 0,00 & 0,00 & 0 & 0 & 0 & 0,05 & 0,00 & 0,00 \\
\hline 21 & & & 0,80 & 0,80 & 2,00 & 4,00 & 6,00 & 1,00 & 0,00 & 0,00 & 0,00 & 0,20 & 1,00 & 1,00 & $-1,00$ & 0,00 & 0,00 & 0 & 0 & 0 & 0,05 & 0,00 & 0,00 \\
\hline 22 & & & 0,85 & 0,85 & 2,00 & 4,00 & 6,00 & 1,00 & 0,00 & 0,00 & 0,00 & 0,15 & 1,00 & 1,00 & $-1,00$ & 0,00 & 0,00 & 0 & 0 & 0 & 0,05 & 0,00 & 0,00 \\
\hline 23 & & & 0,90 & 0,90 & 2,00 & 4,00 & 6,00 & 1,00 & 0,00 & 0,00 & 0,00 & 0,10 & 1,00 & 1,00 & $-1,00$ & 0,00 & 0,00 & 0 & 0 & 0 & 0,05 & 0,00 & 0,00 \\
\hline 24 & & & 0,95 & 0,95 & 2,00 & 4,00 & 6,00 & 1,00 & 0,00 & 0,00 & 0,00 & 0,05 & 1,00 & 1,00 & $-1,00$ & 0,00 & 0,00 & 0 & 0 & 0 & 0,05 & 0,00 & 0,00 \\
\hline 25 & & & 1,00 & 1,00 & 2,00 & 4,00 & 6,00 & 0,40 & 0,60 & 0,00 & 0,00 & 0,00 & 1,00 & 1,00 & 0,20 & $-0,60$ & 0,00 & 1 & 0 & 0 & 0,05 & 0,00 & 0,00 \\
\hline 26 & & & 1,05 & 1,02 & 2,03 & 4,00 & 6,00 & 0,40 & 0,60 & 0,00 & 0,00 & 0,01 & 0,97 & 1,00 & 0,20 & $-0,60$ & 0,00 & 0 & 0 & 0 & $-0,01$ & 0,03 & 0,00 \\
\hline 27 & & & $:$ & $:$ & $:$ & $:$ & $:$ & : & $:$ & $:$ & : & : & $:$ & $:$ & $:$ & : & : & $:$ & $:$ & ${ }^{\circ}$ & : & : & : \\
\hline
\end{tabular}
no. 7 , with recursive updates from row no. 6 . Only the sometimes necessary distinction of cases with nested IF and AND constructs seems elaborate.

Figure 3: Time-oriented approach, calculation spreadsheet. 


\section{Time Advance}

\section{$t: \quad \mathbf{D 7}=$ IF ( D6 t_end; D6 + delta_t $)$}

If the previous time is smaller than the maximum time (15 seconds), the actual time is the previous time plus the time step.

Position update: The actual positions are the previous position plus the previous velocity times the time step:

$x_{1}: \mathbf{E 7}=\mathbf{E 6}$ +delta_t $, x_{2}, x_{3}, x_{4}$ alike

Position difference is calculated by differences of positions minus sphere diameter:

$y_{1}: \mathbf{M 7}=\mathbf{F} \mathbf{-} \mathbf{E 7}-\mathbf{d}, \quad y_{2}, y_{3}$ alike

\section{Collisions Detection:}

The difference between previous position difference and actual position difference changes sign, if a collision has just occurred:

delta_y1: X7=M6-M7, delta_y2 and delta y3 alike, the type of collision is given by the impact indicator

s12: U7 = IF ( $M 7<=0 ;$ IF ( X7>0;1;0 ); 0 )

s23 and s34 alike, being ' 1 ' in case of detected collision $C_{1 \times 2}, C_{2 \times 3}$, or $C_{3 \times 4}$ resp.

\section{Collision Handling:}

New Velocity difference:

If the impact number $\mathbf{s} \mathbf{1 2}$ is ' $\mathbf{1}$ ', the velocity difference dy1 is calculated with formula (5) for $C_{1 \times 2}$

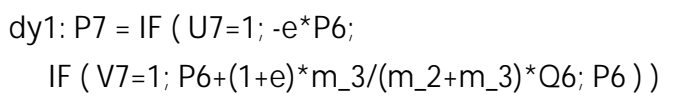

If the impact number $\mathbf{5} \mathbf{2 3}$ is one, the velocity difference dy $\mathbf{3}$ is calculated with formula (5). If both impact numbers $\mathbf{s 1 2}$ and $\mathbf{s} \mathbf{2 3}$ are zero, the actual velocity difference $\mathbf{d y} \mathbf{1}$ is the previous velocity difference dyl (dy2 and dy3 alike).

\section{New Velocity: $\quad$ dx1: $17=$ IF $(U 7=1$; $\left.16+(1+e) * m \_2 /\left(m \_1+m \_2\right) * P 6 ; 16\right)$}

If the impact number $\mathbf{s} \mathbf{1 2}$ is one $\left(C_{1 \times 2}\right)$, the actual velocity $d x 1$ is calculated with formula similar to (5), else the actual velocity is the previous velocity, $\mathbf{d x 2}, \mathbf{d x} \mathbf{3}$ and $\mathbf{d x} \mathbf{4}$ alike, and analogously for collisions $C_{2 \times 3}$. and $C_{3 \times 4}$.

The worksheet for the first task (Figure 3 ) shows the movement for the first 22 timesteps - with step size delta_t of 0.05; the first collision is detected and handled at $t=\mathbf{1}$, indicated by a one for $\mathbf{5 1 2}$ (highlighted in orange.

The columns for the collision indicators allow a simple calculation of the number of collisions: indicated in cell A19 (highlighted in orange): $n_{C}=\operatorname{SUM}(\mathbf{U}: \mathbf{W})$.
The advantage of the time-oriented approach is the flexibility in the model description - instead of the simple linear translational movement for the position update also a nonlinear movement described by ODEs can be used instead of the update a Euler solver for the ODE must be used. The disadvantage of the approach is the fact, that all collisions time instants $t_{c}^{a}$ are detected with a delay at the next grid time instant $t_{k+1}$. The classical interpolation strategy of simulators for event detection could help, but is elaborate: the collision time $t_{c}^{a}$ could be interpolated between $t_{k}$ and $t_{k+1}$.

Most of the up to now 14 published benchmark solutions are based on a simulator, which makes use of an ODE solver with integrated event detection for the collisions, e.g. [2-4].

\subsection{Event-oriented approach}

The event-oriented approach makes use of the special linear movement dynamics given by model (1), which allows to calculate the next collision time instant by means of the intersection formula (7). Each collision is a state event, which changes the velocities of the involved spheres due to collision formulas (5) and (6). From the viewpoint of events, the collision event is an algorithmic state event: intersection formulas and case-by-case analysis constitute the algorithm for the next collision time with the associated collision type.

The implementation in Excel follows the time-oriented approach, in order to provide a better comparability. Again, in rows the variables are calculated, but each row is now associated with the respective collision time. Variables and parameters are:

\begin{tabular}{cccccc} 
tca & \multicolumn{4}{c}{ current absolute collision time $t_{c}^{a}$} \\
tcr & \multicolumn{4}{c}{ current relative collision time $t_{c}^{r}$} \\
$\mathbf{x 1}$ & $\mathbf{x} \mathbf{2}$ & $\mathbf{x}$ & $\mathbf{x} \mathbf{4}$ & $x_{i}\left(t_{k}\right)$ & position of spheres \\
$\mathbf{d x 1}$ & $\mathbf{d x 2}$ & $\mathbf{d x 3}$ & $\mathbf{d x 4}$ & $\dot{x}_{i}\left(t_{k}\right)$ & velocity of spheres
\end{tabular}

y1 y2 y3 $y_{i}\left(t_{k}\right)$ position differences

dy1 dy2 dy3 $\quad \dot{y}_{i}\left(t_{k}\right)$ velocity differences

tcr12 tcr23 tcr34 time until next possible collision

time instant $t_{c, i \times i+1}^{r}$ ('FALSE' if no collision possible) cind collision indicator for next collision type.

Parameters are defined in fixed calls and named:

\section{a, d, v_0, m_1, m_2, m_3, m_4, e, t_0, tend.}

Figure 4 shows the Excel worksheet with formula implementation. The implementation of the model and its formulas is straightforward and briefly sketched for row no. 9 , with recursive updates from row no. 8: 


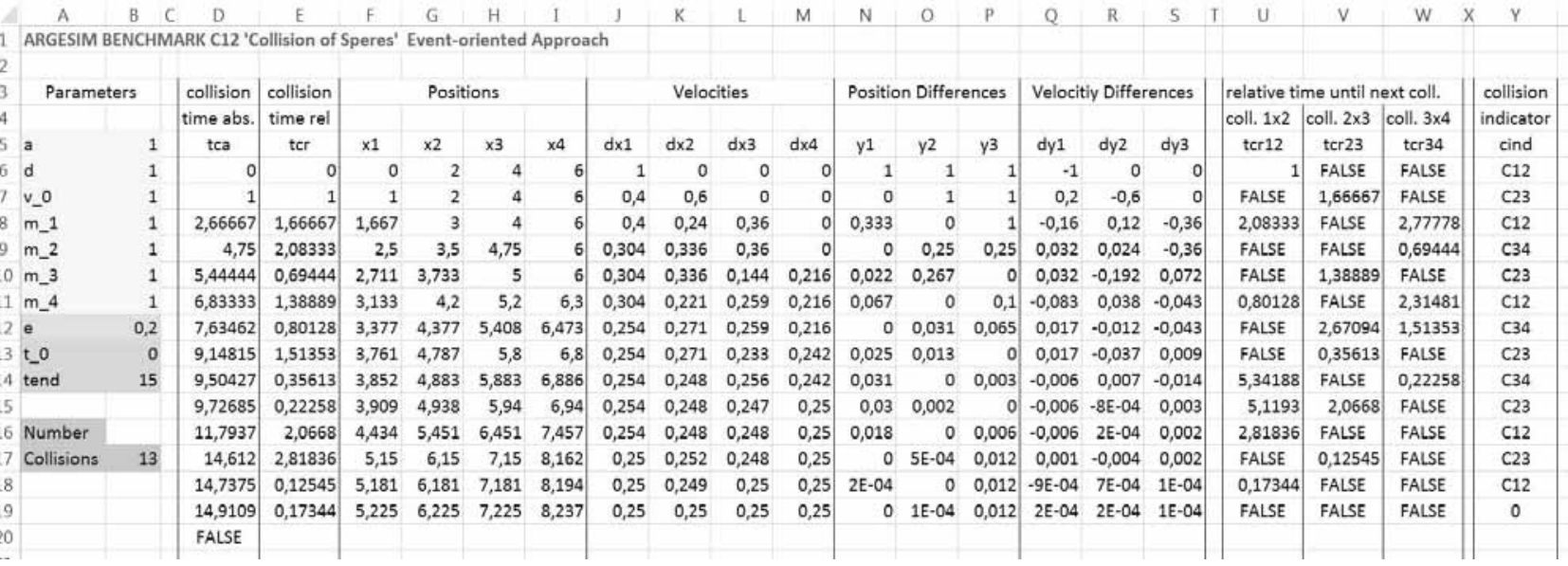

Figure 4: Event-oriented approach, calculation spreadsheet

\section{Collision time advance}

Row update starts with the selection of the next relative collision time span $t_{c, i \times i+1}^{r}$ due to minimum selection in formula (7) by:

\section{tcr: E9 =IF ( D8>t_end; FALSE; MIN( U8:W8 ) )}

If the previous absolute collision time $t_{c}^{a}$ is smaller than the final time, time span $t_{c}^{r}$ for next collision is the minimum of precalculated relative collision times for all possible collisions.

Now the next (actual) absolute collision time $t_{c, k+1}^{a}$ can be calculated by adding the actual (next) relative collision time span $t_{c}^{r}$ to the previous absolute collision time $t_{c, k}^{a}($ formula (7)):

\section{tca: D9 =IF ( D8>tend; FALSE; D8+E9 )}

Position update: The next (actual) positions are the previous position plus the previous velocity times relative collision time: $x_{1}$ : F9=F8 +E9*J8, $x_{2}, x_{3}, x_{4}$ alike.

Position differences are calculated by differences of positions minus sphere diameter:

$$
y_{1}: \mathbf{N} 9=\mathbf{G 9}-\mathbf{F} \text { - } \mathbf{d}, \quad y_{2}, y_{3} \text { alike }
$$

\section{Velocities, velocity differences}

As a collision has happened, velocities and velocity differences must be updated due to formulas (5) and (6) for spheres involved (indicated by precalculated collision indicator cind) or must be simply continued:

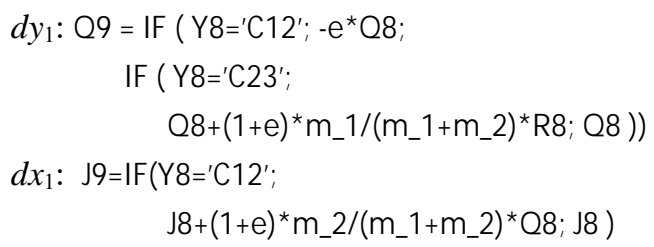

\section{Next collision determination}

The intersection formula (7) allows to calculate the time spans until all next possible collisions

$\ldots t_{c, 1 \times 2}^{r}: \mathbf{U} \mathbf{9}=\mathbf{I F}(\mathbf{N} \mathbf{9}=\mathbf{0}$; FALSE;

\section{IF (Q9>=0; FALSE; N9/-Q9) )}

If the position difference $y_{\mathrm{i}}$ is not zero and the velocity difference $d y_{\mathrm{i}}$ is less than zero, the time span until next collision $t_{c, i \times i+1}^{r}$ is calculated by the position difference $y_{i}$ divided with the negative velocity difference $d y_{i}$.

The collision indicator cind determines, which collision comes next:

\section{Y9 = IF ( U9=E10; 'C12'; IF ( V9=E10; 'C23'; IF ( W9=E10; 'C34'; '0') ) )}

Indeed, there can be maximal two possible next collisions, but there can be also no next (further) collision, so that the sequence of collisions has ended (indicated by type $\mathbf{0} \mathbf{0}$ ).

The number of collisions $n_{C}$ can be easily determined by counting the number of rows with detected collision type or collision time

$$
\begin{aligned}
& \left.n_{C}: \mathbf{B 1 7}=\text { COUNTIF ( Y6:Y29; } \diamond^{\prime} \mathbf{0}^{\prime}\right) \text { or } \\
& n_{C}: \text { B17 =COUNTIF ( D6:D29; > }>0 \text { ) }
\end{aligned}
$$

In general, the implementation is straightforward, only the case-by-case analysis with its nested IF constructs seems to be laborious. The necessary number of rows with recursive updates depends on the number of collisions - which is not known in advance.

For simplicity, a maximal number of rows is implemented, and if collisions stop, collisions type is set to zero, and collision time is set to FALSE. (see Figure 4, row 19 and row 20). 


\section{Task a - Time Domain Analysis}

The first task concentrates on time domain analysis for given values of the collision coefficient: first a classical simulation showing the behaviour with numerous hits, and second, the simulation-based analysis of specific collisions cases -- elastic collisions, quasi-plastic collision, and inelastic collision.

\subsection{Task a1 - Basic time analysis}

The first task requires a graphical representation of the position differences $y_{i}(t)$ for fixed collision coefficient $e=0.2$, and initial velocity of first sphere $\dot{x}_{1}(0)=v_{0}=$ 1 , to be observed on time interval $[0,15]$.

Figure 5 and Figure 6 show the positions and the position differences for the time-oriented approach.

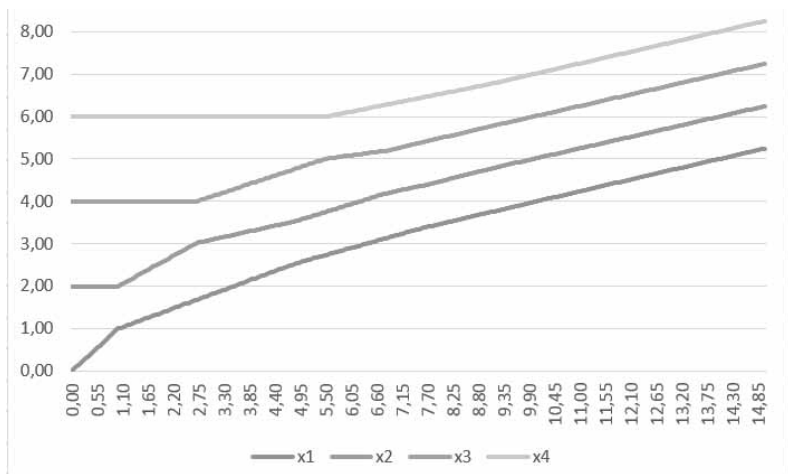

Figure 4: Positions over time - time oriented approach.

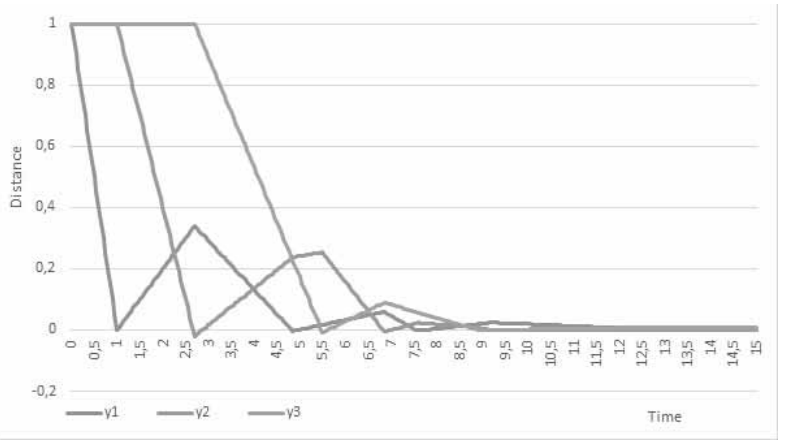

Figure 5: Position differences over time - time oriented approach.

Figure 6 displays the position differences for the eventoriented approach - the time scale grid represents the event times, and interpolation to a synchronous grid shows only small differences to results of the time-oriented approach. A more precise comparison is given in Table 1, which lists the collision times and collision types for both approaches (13 collisions in both approaches). As expected, the collision times in case of the time-oriented approach are delayed: the relative delay is less than the time step, but absolutely the delay is aggregated.

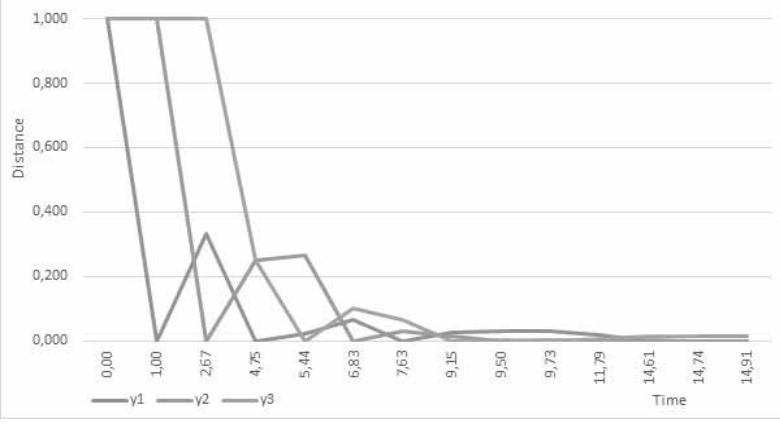

Figure 6: Position differences over collision - event-oriented approach.

\begin{tabular}{|c|c|c|c|}
\hline \multicolumn{2}{|c|}{ Time-oriented Approach } & \multicolumn{2}{|c|}{ Event-oriented approach } \\
\hline $\begin{array}{l}\text { Collision } \\
\text { time }\end{array}$ & $\begin{array}{c}\text { Collision } \\
\text { Type }\end{array}$ & $\begin{array}{l}\text { Collision } \\
\text { time }\end{array}$ & $\begin{array}{c}\text { Collision } \\
\text { Type }\end{array}$ \\
\hline 1 & $1-2$ & 1 & $1-2$ \\
\hline 2.7 & $2-3$ & 2.67 & $2-3$ \\
\hline 4.85 & $1-2$ & 4.75 & $1-2$ \\
\hline 5.5 & $3-4$ & 5.44 & $3-4$ \\
\hline 6.85 & $2-3$ & 6.83 & $2-3$ \\
\hline 7.6 & $1-2$ & 7.63 & $1-2$ \\
\hline 8.95 & $3-4$ & 9.15 & $3-4$ \\
\hline 9.2 & $2-3$ & 9.5 & $2-3$ \\
\hline 9.25 & $3-4$ & 9.73 & $3-4$ \\
\hline 9.3 & $2-3$ & 11.79 & $2-3$ \\
\hline 13.05 & $1-2$ & 14.61 & $1-2$ \\
\hline 13.1 & $2-3$ & 14.47 & $2-3$ \\
\hline 13.2 & $1-2$ & 14.91 & $1-2$ \\
\hline
\end{tabular}

Table 1: Comparison of collision times - time-oriented vs. event-oriented approach.

A realistic collision takes time. This fact is not taken into account in the event-oriented solution. However, in the time-oriented solution this impact time is implicitly taken into account by the delay error. Because of this, the solution of the time-oriented approach seems to be more realistic.

\subsection{Task a2 - elastic vs. quasi-plastic case}

This task requires the simulation for special cases of the collision coefficient $e-$ the elastic case, and the quasiplastic case.

Elastic case. In the elastic case with $e_{e l}=1$ each collision gives the full moment to the sphere hit, so that the hitting sphere stops, and the sphere hit continues with full velocity. Simple analytical considerations derive the results for the final velocities: the first three spheres are stopped and do not move, so that $x_{i}($ tend $)=0, i=1,2,3$, and the $4^{\text {th }}$ sphere moves on with the initial velocity $\left(x_{4}(\right.$ tend $\left.)=v_{0}=1\right)$. Simulation results (Table 2$)$ coincide with the expected values, also for the time-oriented approach, because the collision time instants of the eventoriented approach are a subset of the discrete time grid for the chosen resolution $\Delta \mathrm{t}=0.05$. 


\begin{tabular}{c|c|c}
$\begin{array}{c}\text { Final Velocities } \\
\text { elastic case }\end{array}$ & $\begin{array}{c}\text { Time-oriented } \\
\text { Approach }\end{array}$ & $\begin{array}{l}\text { Event-oriented } \\
\text { approach }\end{array}$ \\
\hline$x_{i}($ tend $), i=1,2,3$ & 0 & 0 \\
\hline$x_{4}($ tend $)$ & 1 & 1
\end{tabular}

Table 2: Final velocities for elastic collisions.

Quasi-plastic case. While elastic collisions and inelastic collision represent generic cases, the quasi-plastic case lies in-between: all spheres move after some time and after some collisions with the same velocity. These movements with same velocity are result of a proper chosen collision coefficient $e_{q p}$, which forwards energy at sufficient many collisions in that amount, that i) all spheres move with same velocity, and so that ii) no further collisions occur. The proper sequence of collisions results in a dynamic equilibrium.

Simple guess and variation of the collision coefficient could give sufficient accurate approximation of the value for $e_{q p}$. Simulation trials indicate, that an appropriate collision coefficient must be small -- below 0.25 - and the number of collisions rises above 10 .

From algorithmic viewpoint, the search for an appropriate collision coefficient $e_{q p}$ is an optimisation problem, or boundary problem, resp. The boundary value, or the goal function, resp., is expressed in terms of same velocities for all spheres, or equivalently with zero velocity differences for all spheres. An appropriate goal function is for instance:

$$
F(e)=\sum_{i=1}^{3}\left|y_{i}\left(t_{\text {end }}\right)\right| \rightarrow 0
$$

Excel provides as standard feature the Goal Seeking Function in the What If Analysis - suitable for approximating the collision coefficient value $e_{q p}$ for the quasiplastic case. Figure 7 shows the spreadsheet for this approximation. The cells B2O-B23 contain the final velocities, cells B25-B27 the final velocity differences, and cell

B28 defines the goal function (10):

\section{$B 28==A B S(B 25)+A B S(B 26)+A B S(B 27)$}

The Goal Seeking Function needs as input the cell of the parameter to be iterated - the value of the collision coefficient $e$ in cell B12, the goal function evaluation - formula (10) in B28, and the goal value - zero in this case. The Goal Seeking Function results in values given in Figure 7 (event-oriented approach), with tuning parameters of a maximum of 1000 iteration steps and a deviation of 0.0001 .

\begin{tabular}{|c|c|c|c|c|c|c|}
\hline 4 & A & B & C & D & E & $\mathrm{F}$ \\
\hline 1 & \multicolumn{6}{|c|}{ ARGESIM BENCHMARK C12 'Collision of Speres' Event-oriented Approacl } \\
\hline 2 & & & & & & \\
\hline 3 & \multicolumn{2}{|c|}{ Parameters } & & collision & collision & Positions \\
\hline 4 & & & & time abs. & time rel & \\
\hline 5 & a & 1 & & tca & tcr & $\mathrm{x} 1$ \\
\hline 6 & d & 1 & & 0 & 0 & 0 \\
\hline 7 & v_o & 1 & & 1 & 1 & 1 \\
\hline 8 & m_1 & 1 & & 2,6896588 & 1,6896588 & 1,6896588 \\
\hline 9 & m_2 & 1 & & 4,5524703 & 1,8628116 & 2,4499924 \\
\hline 10 & m_3 & 1 & & 5,5446056 & 0,9921353 & 2,7571235 \\
\hline 11 & m_4 & 1 & & 6,6222959 & 1,0776903 & 3,0907395 \\
\hline 12 & e & 0,1836709 & & 7,3578167 & 0,7355208 & 3,3184316 \\
\hline 13 & t_o & 0 & & 7,9460849 & 0,5882682 & 3,4705597 \\
\hline 14 & tend & 15 & & 8,338899 & 0,392814 & 3,5721426 \\
\hline 15 & & & & 8,5554333 & 0,2165344 & 3,6281391 \\
\hline 16 & \multicolumn{2}{|c|}{ Number Collisions } & & 9,4994705 & 0,9440372 & 3,8722702 \\
\hline 17 & & 17 & & 9,6974983 & 0,1980278 & 3,9234808 \\
\hline 18 & & & & 9,7075366 & 0,0100383 & 3,9260031 \\
\hline 19 & \multicolumn{2}{|c|}{ Final Velocities } & & 9,7198529 & 0,0123163 & 3,9290978 \\
\hline 20 & $\mathrm{dx} 1$ & 0,2500068 & & 11,378927 & 1,659074 & 4,344147 \\
\hline 21 & $\mathrm{dx} 2$ & 0,2499916 & & 11,688162 & 0,3092356 & 4,4215081 \\
\hline 22 & $\mathrm{~d} \times 3$ & 0,250012 & & 12,186975 & 0,4988126 & 4,5462957 \\
\hline 23 & $\mathrm{dx} 4$ & 0,2499896 & & 14,133557 & 1,9465822 & 5,0332705 \\
\hline 24 & \multicolumn{2}{|c|}{ Goal Function } & & FALSE & & \\
\hline 25 & dy1 & $1,518 \mathrm{E}-05$ & & & & \\
\hline 26 & dy2 & $-2,035 \mathrm{E}-05$ & & & & \\
\hline 27 & dy3 & $2,232 \mathrm{E}-05$ & & & & \\
\hline 28 & sum_dy & $5,784 \mathrm{E}-05$ & & & & \\
\hline
\end{tabular}

Figure 7: Spreadsheet for goal seeking of restitution coefficient for quasi-plastic case - event-oriented approach.

\begin{tabular}{l|c|c} 
Approach & Time-oriented & Event-oriented \\
\hline $\begin{array}{l}\text { Collision } \\
\text { Coefficient }\end{array}$ & $e_{q p}=0.191$ & $e_{q p}=0.184$ \\
\hline $\begin{array}{l}\text { Final } \\
\text { Velocities }\end{array}$ & $x_{i}($ tend $)=0.25$ & $x_{i}($ tend $)=0.25$ \\
\hline Collisions & 17 & 17
\end{tabular}

Table 3: Collision coefficient for quasi-plastic case results of Goal Seeking tool.

Table 3 confronts the results for the two modelling approaches: the event-oriented approach results in a smaller coefficient $e_{q p}=0.184$ than in the time-oriented approach with $e_{q p}=0.191$ - caused by the delayed collision times of the time-oriented approach. But both approaches manage 17 collisions until the quasi-plastic equilibrium.

It is evident, that the inelastic case (plastic case) with $e_{p l}=0$ results in spheres moving with same velocity. If the first sphere hits the second, both stick together and move on with half velocity - but double mass !, etc., so that after three collisions all spheres stick together and move with same velocity. The implementation does not regard this special case with 'increasing' mass. On the other hand, the inelastic case happens, if the collision coefficient is decreased until zero - with theoretically infinite number of collisions - see results Task b1. 


\section{Task $b$ - Dependence on Collision Coefficient}

This task analyses the dependence of the number of collisions and of the final velocities on the collision coefficient. Numerical problems for small values of the collision coefficient can be expected. Variation of the collision coefficient $e$ in cell $\mathbf{B} 12$ requires recalculation of the spreadsheet - here Excel macros are of help, which vary the collision coefficient, perform a spreadsheet update calculation, and write results into columns.

\subsection{Task b1 - Number of collisions}

It is known, that the elastic case $e_{e l}=1$ produces three consecutive collisions $\left(n_{C}=3\right)$ - but with decreasing collision coefficient $e<1$, multiple collisions occur, so that the number of collisions is increasing $\left(n_{C}>3\right)$.

A simple Excel macro helps to determine the number of collisions $n_{C}(e)$ dependent on the collision coefficient and stores results (Figure 8):

i) increase of $e$ by increment 0.001 in cell B12, and store value in consecutive cell of $x$-axis columns AAn for varying $e$ (start with $e=0.15$ ),

ii) update spreadsheet,

iii) store resulting number of collisions $n_{C}$ from cell B17 in consecutive cell of $y$-axis column AFn for storing function $n_{C}(e)$

Figure 8 shows the results for $n_{C}(e)$ numerically in columns AAn and AFn, and Figure 9 plots the corresponding graph $n_{C}(e)$ - using the event-oriented approach. For a collision coefficient $e>0.54$, only three collisions are occuring $\left(n_{C}=3\right)$. With decreasing collision coefficient $e<0.54$ the number of collisions $n_{C}(e)$ is rising, for $e \rightarrow$ 0 the number theoretically increases monotonically to infinity: $n_{C} \rightarrow \infty$. However, there is a limit in reducing the collision coefficient due to general numerical computation limitations - for values $e<0.15$ computations fail.

The event-oriented approach is able to calculate up to $n_{C}=48$ collisions for a value of $e=0.17$, for values below $e<0.17$ the results are not reliable (number of collisions $n_{C}(e)$ seems to decrase) or simply fail.

The time-oriented approach - plot in Figure 10 - is faced with an additional erroneous behaviour. Because of the delay in collision detection, collisions times not only are delayed, but collisions themselves may vanish, if they are to near to the previous ones. As consequence, the time-oriented approach is able to calculate only up to $n_{C}=20$ collisions for a value of $e=0.18$; for values below $e<0.18$ the computations fail.

\begin{tabular}{|c|c|c|c|c|c|c|c|c|c|}
\hline 2 & A & B & $C Z$ & - $A A$ & $A B$ & $A C$ & $A D$ & $\mathrm{AE}$ & $\mathrm{AF}$ \\
\hline 1 & \multicolumn{9}{|c|}{ ARGESIM BENCHMARK C12 'Collision of Speres' Event-oriented Approach } \\
\hline 3 & \multicolumn{2}{|c|}{ Parameters } & \multicolumn{2}{|r|}{ Varation } & \multicolumn{4}{|c|}{ Final Velocities } & $\begin{array}{l}\text { number } \\
\text { collisions }\end{array}$ \\
\hline 5 & a & 1 & & e & dx1 & dx2 & $\mathrm{dx3}$ & $\mathrm{dx} 4$ & $\mathrm{nC}$ \\
\hline 6 & d & 1 & & 0,15 & 0,25 & 0,25 & 0,25 & 0,25 & 44 \\
\hline 7 & v_o & 1 & & 0,16 & 0,25 & 0,25 & 0,25 & 0,25 & 48 \\
\hline 8 & m_1 & 1 & & 0,17 & 0,25 & 0,25 & 0,25 & 0,25 & 48 \\
\hline 9 & m_2 & 1 & & 0,18 & 0,25 & 0,25 & 0,25 & 0,25 & 21 \\
\hline 10 & m_3 & 1 & & 0,19 & 0,25 & 0,25 & 0,25 & 0,25 & 13 \\
\hline 11 & m_4 & 1 & & 0,20 & 0,25 & 0,25 & 0,25 & 0,25 & 13 \\
\hline 12 & e & 1 & & 0,21 & 0,25 & 0,25 & 0,25 & 0,25 & 9 \\
\hline 13 & t_o & 0 & & 0,22 & 0,25 & 0,25 & 0,25 & 0,25 & 9 \\
\hline 14 & tend & 15 & & 0,23 & 0,25 & 0,25 & 0,25 & 0,25 & 9 \\
\hline 15 & & & & 0,24 & 0,24 & 0,26 & 0,26 & 0,24 & 6 \\
\hline 16 & Number & & & 0,25 & 0,24 & 0,26 & 0,26 & 0,24 & 6 \\
\hline 17 & Collision & 3 & & 0,26 & 0,24 & 0,26 & 0,26 & 0,25 & 6 \\
\hline 18 & & & & 0,27 & 0,24 & 0,25 & 0,25 & 0,26 & 6 \\
\hline : & & & & $:$ & $:$ & $:$ & $:$ & $:$ & : \\
\hline 83 & & & & 0,92 & 0,04 & 0,04 & 0,04 & 0,88 & 3 \\
\hline 84 & & & & 0,93 & 0,03 & 0,03 & 0,03 & 0,90 & 3 \\
\hline 85 & & & & 0,94 & 0,03 & 0,03 & 0,03 & 0,91 & 3 \\
\hline 86 & & & & 0,95 & 0,02 & 0,02 & 0,02 & 0,93 & 3 \\
\hline 87 & & & & 0,96 & 0,02 & 0,02 & 0,02 & 0,94 & 3 \\
\hline 88 & & & & 0,97 & 0,01 & 0,01 & 0,01 & 0,96 & 3 \\
\hline 89 & & & & 0,98 & 0,01 & 0,01 & 0,01 & 0,97 & 3 \\
\hline 90 & & & & 0,99 & 0,00 & 0,00 & 0,00 & 0,99 & 3 \\
\hline 91 & & & & 1,00 & 0,00 & 0,00 & 0,00 & 1,00 & 3 \\
\hline
\end{tabular}

Figure 8: Spreadsheet with calculation of collisions number final velocities dxi(e) for varying collision coefficient e - event-oriented approach.

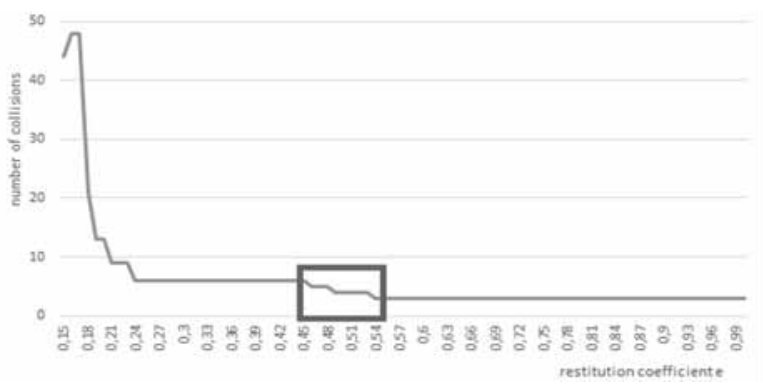

Figure 9: Number of collisions as function of collision coefficient - time-oriented approach.

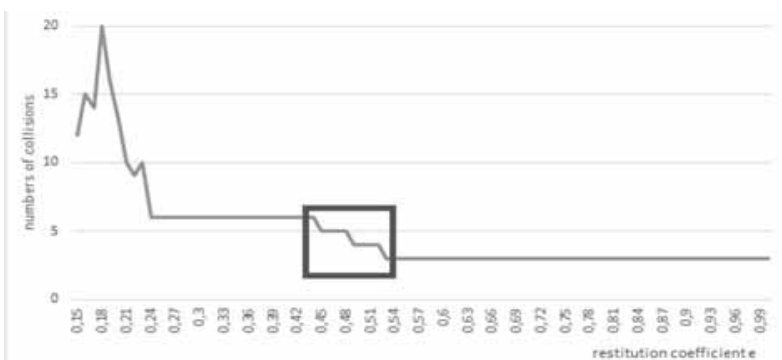

Figure 10: Number of collisions as function of collision coefficient - time-oriented approach.

Delay error and vanishing collisions result in another erroneous phenomenon: the function $n_{C}(e)$ is not monotonous - in Figure 10, for $e \sim 0.22$ the number of collisions seem to decrease (not only below the 'numerical' border $e<$ 0.18). A smaller time step does not really help, because it only shifts the errors in relation to the time step decrease. 
Both approaches coincide with the increase of the collision number from $n_{C}(e)=3$ to $n_{C}(e)=6$ within the range of $e=0.54$ down to $e=0.45$, simulating correctly the first re-hits of the spheres (indicated by green rectangles in Figure 9 and Figure 10).

For $e \rightarrow 0$, the number of collisions is going to infinity, so that from this viewpoint the plastic case, or the inelastic case resp., is associated with an infinite number of collisions: the spheres do not stick together and move as 'increased' mass, the spheres get infinitely near to each other. As consequence, a proper model for the plastic case must follow the strategy of three collisions with increasing mass of 'multiple' spheres.

The number of collisions indeed is increasing drastically. A benchmark solution with a high-accuracy computation tool ([5]) results in $n_{C}(e)=11216$ for a collision coefficient of $e=0.1715729$. Interestingly, the number of collisions does not really matter for very small values of the collision coefficient - in any case, the result is the quasi-plastic case; for instance, another high-accuracy benchmark solution ([6]) reaches a minimal collision coefficient of $e=0.0811$ (with limiting the collision number in the decreasing loop).

\subsection{Task b2 - Final velocities}

Task $1 \mathrm{~b}$ already investigated the final velocities of the spheres for the elastic case and for the quasi-plastic case. For further analysis, the final velocities are now investigated as function of the collision coefficient $e$. The development of $\dot{x}_{i}(e)$ is expected to be within the range of the elastic case $-\dot{x}_{i}(e=1)=0, \dot{x}_{4}(e=1)=1-$ and the quasi-plastic case $-\dot{x}_{i}(e=0.184)=0.25$.

As before, a simple Excel macro determines the final velocities $\dot{x}_{i}^{\text {tend }}(e)$ dependent on the collision coefficient and stores results. Figure 8 shows implementation and the numerical results, Figure 11 plots the functions $\dot{x}_{i}^{\text {tend }}(e)$ generated by the event-oriented approach.

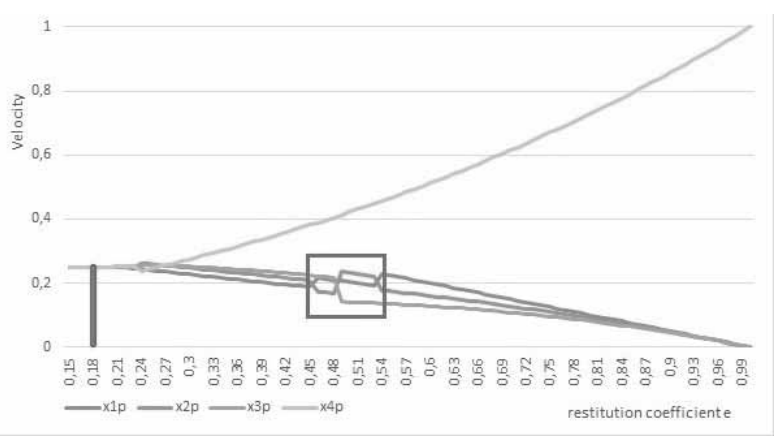

Figure 11: Final velocity of each sphere as function of collision coefficient - event--oriented approach.

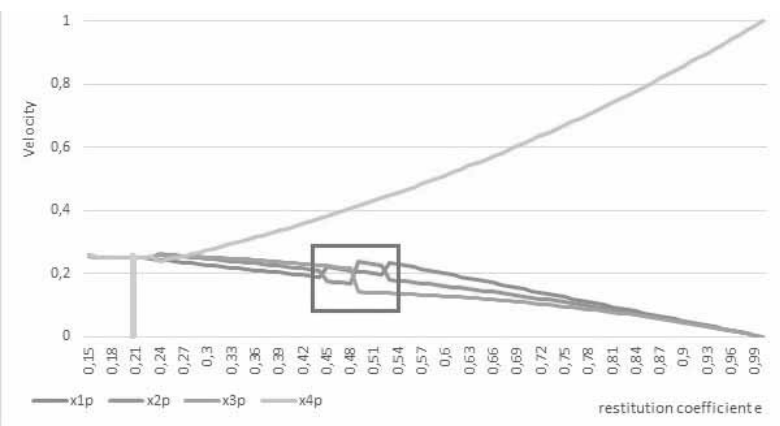

Figure 12: Final velocity of each sphere as function of collision coefficient - time-oriented approach.

The results for the time-oriented approach in Figure 12 show almost no difference to the results of the event-oriented approach in Figure 11. The final velocities of the first three spheres $\dot{x}_{i}^{\text {tend }}(e)$ tend towards zero with an increasing collision coefficient $e$, and the final velocity of the fourth sphere $\dot{x}_{4}^{\text {tend }}(e)$ rises until one with increasing collision coefficient.

For decreasing collision coefficient all velocities tend towards the value $\dot{x}_{i}^{\text {tend }}\left(e_{q p}\right)=0.25$ and reach the value at the slightly different collision coefficients for the quasi-plastic collision case (red bar in Figure 11 for event-oriented approach, yellow bar in Figure 12 for the time-oriented approach). Interestingly, while the calculations for the number of collisions for collision coefficients below 0.15 fail in both approaches (see Figure 9 and Figure 10), calculations for the final velocities do not fail as given in Figures 11 and Figure 12: although the number of collisions may vary chaotically, the associated changes of velocities are below any significant values.

Both approaches coincide in the changes of the velocities within the range of $e=0.54$ down to $e=0.45$, in accordance with the increase of the number of collisions from $n_{C}(e)=3$ to $n_{C}(e)=6$ (indicated in figures 9-12 by the green rectangle).

\section{Task c - Boundary Value - Stochastic Parameters}

In most benchmarks, the last task is a challenging one: complex experiments, sophisticated experiment control, model extensions, etc. Also Benchmark $\mathrm{C} 12$ requires in the third task elaborate experiments with the model: a boundary value problem, and statistical parameter analysis. But spreadsheet tools offer features for such tasks as standard features. Here the various data analysis features play an important role. 


\subsection{Task c1 - Velocity boundary value}

This task requests the calculation of the collision coefficient $e_{v_{0} / 2}$ which results in half initial velocity for the final velocity of the fourth sphere: $\dot{x}_{4}^{\text {tend }}\left(e_{v_{0} / 2}\right)=\frac{v_{0}}{2}=0.5$. Excel provides as standard feature the Goal Seeking Function in the What If Analysis - suitable for approximating the collision coefficient value $e_{v_{0} / 2}$. Analogously to task b2 a goal function has to be defined, in this case simply

$$
\left.F(e)=\left|\dot{x}_{4}^{\text {tend }}-\frac{v_{0}}{2}\right| \rightarrow 0 \quad \text { B28 = =ABS ( B25 - v_0 }\right)
$$

The Goal Seeking Function needs as input the cell of the parameter to be iterated $-e$ in cell $\mathbf{B 1 2}$, the goal function evaluation - the above formula in B28, and goal value zero. The Goal Seeking Function gives as result the value $e_{v_{0} / 2}=0.587$ for the event-oriented approach, with four collisions (first re-hit of $C_{1 \times 2}$ as fourth collisions). With accuracy limited to three digits, the time-oriented approach gives the same result.

\subsection{Task c2 - Statistical analysis}

This task extends to some extent task b2, calculation of the dependence of the final velocity on the collision coefficient. With given normally distributed stochastic values for the collision parameter $e_{\mu, \sigma}$, the challenge is to calculate the distribution function, the mean value, the standard deviation and the confidence interval (with confidence probability of 95\%) for the final velocity $\dot{x}_{4}^{\text {tend }}\left(e_{\mu, \sigma}\right), \mu=$ $0.5, \sigma=0.05$ for a sufficient large sample size.

Excel provides many statistical functions, and again Excel Macros perform the statistical parameter variation and the storage of the final velocities in the spreadsheet (similar as in Figure 7). The stored sample for the final velocities is then statistically analysed by standard features resulting in the following values:

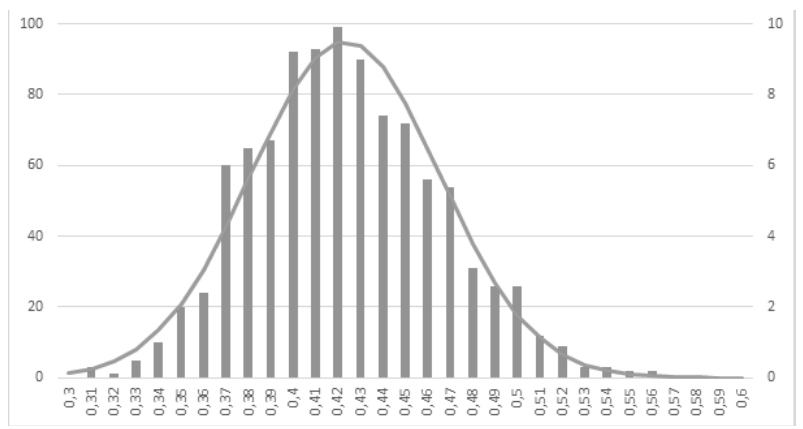

Figure 13: Histogram of the final velocities of the fourth sphere for normal distributed collision coefficient - event-oriented approach.
Mean value: $\ldots \mu\left(\dot{x}_{4}^{\text {tend }}\right)=0.42313 \ldots$.

Standard deviation: $\quad \sigma\left(\dot{x}_{4}^{\text {tend }}\right)=0.041907805$

Confidence interval: [0.420532527 $\leq \mu \leq 0.425727473$ ]

Excel graphic features offer bar charts, used for Figure 13, the histogram for the final velocities of the fourth sphere. Additionally, the formula for the density function allows to plot the distribution approximation (orange curve in Figure 13). With accuracy limited to three digits, the time-oriented approach gives the same result.

\section{Conclusion}

A spreadsheet tool is definitely not a simulator - modelling features for ODEs, processes, events, etc. are missing. But spreadsheet programs are an excellent experiment environment with statistical analysis, optimisation, what-if analysis, date handling, etc. Of course, macros and external programming could be used, but to some extent the standard features allow to implement discrete dynamic processes - recursive formulas for the development of states along with the flow of time with sufficient time resolution.

It is also possible to implement event-driven dynamics, by calculating the time advance due to state-dependent conditions. This implementation technique is used for the event-oriented approach in this benchmark study. A general disadvantage is the lack of accuracy in the Excel standard configuration - possible but laborious to increase. On the other hand, a spreadsheet tool is a very suitable tool for education, so that this $\mathrm{C} 12$ benchmark study is mainly intended for educational use.

\section{References}

[1] Hohmann R, Gotzel C, Pöge C. Comparison 12: Collision Processes in Rows of Spheres, Definition. SNE vol. 9(3) (SNE 27), p. 36-37, 1999.

[2] Hohmann R, Gotzel C, Pöge C. Comparison 12 - ACSL: Hybrid Approach. SNE vol. 9(3) (SNE 27), p. 38, 1999.

[3] Filippov A, Kornev A. C12 Spheres' CollisionAnyLogic: Numerical simulation / Time-oriented Model. SNE vol. 11(2/3) (SNE 32/33), p. 50, 2001.

[4] Lingl M. Comparison 12 - MATLAB/SIMULINK: Continuous Approach - State Events. SNE vol. 9(3) (SNE 27), p. 39, 1999.

[5] Gotzel C, Hohmann R, Pöge C. Schwerdt J. C12 Spheres'Collision - SLX /LEDA: Analytical Simulation / Event-oriented Model. SNE vol. 11(1) (SNE 31), p. 31, 2001.

[6] Willensdorfer M, Breitenecker F. Sphere's Collision FORTRAN Algorithmic Simulation / Time-oriented Model. SNE vol. 11(1), (SNE 31), p.36, 2001. 


\title{
Educational Use of the ARGESIM Benchmarks for Modelling Approaches and Simulation Implementations
}

\author{
Felix Breitenecker ${ }^{1 *}$, Andreas Körner ${ }^{1}$, Horst Ecker ${ }^{2}$ \\ ${ }^{1}$ Mathematical Modelling and Simulation Group, Inst. of Analysis and Scientific Computing, ${ }^{2}$ Inst. of Mechanics \\ and Mechatronics; TU Wien, Wiedner Hauptstrasse 8-10, 1040 Vienna, Austria; felix.breitenecker@tuwien.ac.at
}

SNE 28(4), 2018, 215 - 218, DOI: 10.11128/sne.28.bne.10453

Received: November 15, 2018;

Accepted: November 30, 2018

SNE - Simulation Notes Europe, ARGESIM Publisher Vienna ISSN Print 2305-9974, Online 2306-0271, www.sne-journal.org

Abstract. This short note in SNE 28(4) sketches educational aspects of the 'ARGESIM Benchmarks for Modelling Approaches and Simulation Implementations' published since 1990 in SNE. Interestingly, it turned out, that along with the various benchmark publications in SNE, the benchmarks are used as examples, case studies, and lab work in simulation education in academia. Indeed, most of the benchmarks are a very useful basis for education in modelling and simulation. This contribution lists shortly the educational benefits of each benchmark - a detailed investigation of the educational aspects of the benchmarks, together with a description of classification and development of the benchmarks is scheduled for SNE 29 (1), March 2019.

\section{Introduction}

The ARGESIM Benchmarks for Modelling Approaches and Simulation Implementations are a success story in the simulation community. With up to now 24 benchmarks defined, and 354 benchmark solutions, benchmark reports, or benchmark studies published in SNE - Simulation Notes Europe, the scientific membership journal of EUROSIM, the Federation of European Simulation Societies. The benchmark publications have become a forum for information exchange on modelling approaches, simulation implementations, and features of simulation systems.

Interestingly, it turned out, that along with the various benchmark publications in SNE, the benchmarks are used as examples, case studies, and lab work in simulation education in academia. Indeed, most of the benchmarks are a very useful basis for education in modelling and simulation, at modelling level, and at implementation level.
The benchmarks may also be used for education in application areas, as introductory case studies in mechanical engineering, electrical engineering, economics, biology, etc.

This contribution presents shortly the publication possibilities for benchmark publications generally and for education-oriented benchmark publications (Section 1) and lists the educational benefits of each of the 24 benchmarks (Section 2). The last section comments the future development of new benchmark definitions and benchmark solutions to be initiated.

A detailed investigation of the educational aspects of the benchmarks, together with a description of a classification helpful for education and the development of the benchmarks is scheduled for SNE 29 (1), March 2019 [1].

\section{Benchmark Publications}

ARGESIM, a non-profit organisation for information and publication on simulation, started in 1990 the series $A R$ GESIM Comparisons of Simulation Software with modelling and simulation tasks based on relatively simple, easily comprehensible processes. In the beginning, simulationists were invited to prepare a comparison solution to be published in SNE as one-page solution. Along with development of system simulation, also the Comparisons of Simulation Software developed further on towards the ARGESIM Benchmarks for Modelling Approaches and Simulation Implementations.

In 2006 and in 2018, re-organisations of the publication strategy improved the publication possibilities for benchmark publications, including benchmark publications with emphasis on education:

- Extended definitions of new benchmarks:

SNE introduces new benchmarks with extended scope and indication of educational aspects, e.g. comparing modelling and simulation paradigms, dealing with more complex experiments, and formulating models and tasks especially for educational use - indicating educational aspects, as with benchmarks C19, C20, C21 and planned benchmark C22. 
- Revised definitions of previous benchmarks:

SNE is publishing revised definitions of previous comparisons, updating models, extending the scope, and underlining the educational aspects - in order to continue them as new benchmark also for benchmark contributions with emphasis on education, e.g. C12.

The benchmark publications now may have different content, structure, emphasis, and length - and the authors may underline educational aspects, or may shape the benchmark contribution for use in education. Modellers, simulationists, and lecturers in modelling and simulation are invited to take the challenge to prepare, realise and submit a

- Benchmark Solution with concise description of model implementation and experimentation tasks (two pages SNE), or a

- Benchmark Report with sufficient detailed description of model implementation with variants and adequate experiment formulations (four to six pages SNE), or an

- Educational Benchmark Report, a benchmark report with educational aspects or generally addressing use in education (four to six pages SNE), or a

- Benchmark Study presenting e.g. different / alternative / comparative modelling approaches and sketching analysis variants or supplemental model experiments (six to ten pages SNE), or an

- Educational Benchmark Study, a benchmark study for use in education, or at least with essential educational aspects (six to twelve pages SNE) - see e.g. contribution [2] in this SNE.

\section{Benchmarks for Education}

In the following the up to now defined comparisons and benchmarks are briefly investigated with respect to their suitability for educational purposes:

- C1 Lithium-Cluster Dynamics, SNE 0(1), 1990, is based on three coupled stiff ODEs describing a process in surface physics. Simulation beginners can learn to deal with stiff systems, to perform logarithmic parameter variations, and calculate steady states - using a simulation tool or a calculation tool.

- C2 Flexible Assembly System, SNE 1(1), 1991, is an introductory case study for process modelling with six process station around a circular transport belt for pallets. Novices get an introduction into process modelling and simulation, using an appropriate process simulation tool.
- C3 Generalized Class-E Amplifier, SNE 1(2), 1991, describes an RLC circuit with time-varying resistor. The case study is suited for introduction into analogue circuit modelling - by means of general or application-oriented simulation tools.

- C4 Dining Philosophers I, SNE 1(3), 1991, and C10 Dining Philosophers II, SNE 6(3), 1996,

This almost classical process describing the competition for limited resources is a typical case study for education. While $\mathrm{C} 4$ is very generally defined and invites for analysis of any kind, C10 is defined with emphasis on time domain analysis. The benchmarks are very well suited for education, using arbitrary calculation tools.

- C5 Two State Model, SNE 2(1), 1992, is based on two linear ODEs with state-dependent switching parameters and tests state event detection not really suited for education.

- C6 Emergency Department SNE 2(3), 1992, is based on a process model for follow-up treatment in a hospital's emergency department - with real-world data. Novices get an introduction into process modelling and simulation in a health care system, and health care people get an introduction to simulation capabilities - by means of an appropriate process simulation tool.

- C7 Constrained Pendulum, SNE 3(1), 1993, describes the classical pendulum, which hits a pin and continues swinging with switching states. Beginners in simulation can study the handling of simple state events, using an appropriate simulation or also a general calculation tool.

- C8 Canal-and-Lock System, SNE 6(1), 1996, is based on the scheduling of barges moving through a lock. The benchmark mainly tests features of discrete simulators for modelling complex logic, to validate logic through use of deterministic data, and to check for variance-reduction capabilities. The case study may be of educational interest for process modelling, but also for training programming of scheduling - using a process simulation tool, or direct programming.

- C9 Fuzzy Control of a Two Tank System, SNE 6(2), 1996, revised SNE 16(3), 2006, investigates the efficiency of fuzzy control for a simple hydraulic system. This benchmark may be of interest for introduction into fuzzy control, and simulation beginners learn to implement fuzzy structures by means of simulation tools with (easy) or without (laborious) fuzzy control features, or by classical calculation tools (challenging). 
- C11 SCARA Robot, SNE 8(1), 1998, makes use of the implicit model of a 3DOF robot with classic control and control for collision prevention. This benchmark is very suitable for education purposes. Beginners and trained simulationists can learn about implicit equation-base modelling or about physical power-based block-oriented modelling, and students from mechanical engineering get an introduction to mechanical modelling and simulation. Software tools for education range from multibody systems via system simulators to calculation tools.

- C12 Collision of Spheres, SNE 9(3), 1999, allows different approaches for the analysis of movement and for collision of spheres in a row, investigating the impact from elastic to plastic collisions. This benchmark addresses not only system simulation, it is a classical mechanical or physical case study mainly suited for use in education. Students in technical science can learn about collision phenomena, and novices in simulation get knowledge in state event handling. Interestingly, a broad variety of software tools can be used, from physical simulators to spreadsheet tools.

\section{- C13 Crane Crab and Embedded Control,} SNE 11(1), 2001; rev. SNE 17(1), 2007,

is based on modelling and digital control of a crane crab. The discrete control is designed by means of a state space observer, and by state space control. The revised definition of this benchmark outlines the educational aspects: for simulationists, comparison of models, investigation of discrete control elements, and state-dependent security interventions; and for students in control engineering, modelling and simulation of plant, observer, digital control and security intervention events. Appropriate tools are system simulators and control design systems.

- C14 Supply Chain Management, SNE 11(2-3), 2001, is based on a simplified supply chain (four factories, four distributors, a group of wholesalers, and 12 products). The benchmark concentrates on discrete modelling of the process, especially of the order flow, and on implementation of different order strategies. This benchmark is a classical case study for supply chain modelling, showing also the bullwhip effect dependent on the order strategies - an interesting topic for basic education in production and logistics. The implementation does not really need a simulation system, classical recursive calculations can manage all tasks, so that the benchmark is open as educational case study in many areas.
- C15 Clearance Identification, SNE 12(2-3), 2002, is based on the dynamics of renal clearance. The benchmark puts emphasis on identification of a compartmental model for the clearance using real-word measurement protocols, and on statistical model parameter analysis based on artificial protocols. This benchmark allows to study different identification approaches for a linear dynamic system, e.g. use of sensitivity functions or use of analytical solutions, and to handle artificial data protocols for extended experiments - a useful basis for teaching of both topics, independent of the physiological background. Software tools range from compartmental modelling systems to calculation tools.

- C16 Restaurant Business Dynamics, SNE 14(1), 2004, addresses modelling, simulation and optimisation of a discrete dynamic system. The business system under examination is restaurant operating: depending on financial results (from guests living in the neighbourhood), restaurants open branches in an adequate surrounding or close down. Strategic parameters are tax rate and structural distance to new branch restaurants - to be investigated and optimised in an adequate discrete (stochastically influenced) model (process model, agent-based model, statistical analysis, event model, etc.). This benchmark addresses not only the simulation community, it is an interesting calculation task for everybody, and can be approached in various ways. Consequently, the benchmark is a basis for various education areas, and also suited for spreadsheet calculation.

- C17 Spatial Dynamics of SIR Epidemics, SNE 14 (2/3), 2004; revised SNE 25(1), 2015, investigates a classical population model for the spread of infection diseases (SIR model) and an inhomogeneous spatial approach using cellular automata. This benchmark is very suitable for educational purpose: in modelling, macroscopic and microscopic approaches can be compared, and modelling with advanced cellular automata can be studied; in implementation, discrete interventions (vaccinations) are to be managed properly for comparative investigations. And in application - spread of disease and vaccination - the benchmark is a powerful basis for learning the benefits of modelling and simulation.

- C18 Neural Networks vs. Transfer Functions, SNE 15(1), 2005,

studies alternative approaches for identification of the nonlinear dynamical relation between muscle force 
and muscle-belly thickening. Classical discrete transfer function models and as alternatives neural net models are to be compared. This benchmark may be of educational use for control modelling, and for introduction into neural net modelling and simulation software tools supporting digital control and neural net structures are recommended.

- C19 Pollution in Groundwater Flow, SNE 15(2-3), 2005, revised SNE 16(3-4), 2006,

analyses a homogeneous ground water body with a singular pollution source and with facilities for reduction of contamination, based on the transport equation. The benchmark addresses quite different modelling approaches and solution techniques, from classical discretisation methods via FEM to alternatives techniques like cellular automata, Monte-Carlo methods and Random Walk. The recent revised definition outlines the educational aspects: modelling methods for distributed systems, introduction into instationary distributed diffusion processes with comparative modelling and simulation implementation.

- C20 Complex Production System, SNE 21(3/4), 2011 is intended as a foundation for analyzing manufacturing control systems, by comparing different simulation techniques and/or control algorithms regarding complexity and dynamics. The benchmark is based on two dimensions, defining a total of twelve different scenarios that differ in their complexity and dynamic behaviour. This benchmark is a challenge for expert simulationists to test discrete process simulation systems.

- C21 State Events and Structural-dynamic Systems, SNE 26(2), 2016.

In this recent benchmark, three case studies compare modelling and implementation of state events in dynamic systems, up to structural-dynamic systems. The case studies are: the almost classical bouncing ball dynamics, a switching RLC circuit with different diode models, and the rotating pendulum with free falling phase changing dynamics from swinging to falling (and vice versa) - switching between different degrees of freedom. All three case studies are coming along with educational aspects for simulation methodology, implementation, and application. In application, the benchmark addresses mechanical engineering and electrical engineering, in methodology and implementation the benchmark deals with appropriate description and handling of hybrid and structural-dynamic systems. Depending on the case studies, the benchmark is suited for all educational levels in simulation.
- C22 Pitfalls in Discrete Event Handling, 2019/2020. This planned benchmark will discuss the proper modelling and algorithmic implementation of discrete events, emphasizing in application on flip-flops. As with recent and new benchmarks, educational aspects will be integrated from the beginning.

- CP1 Parallel Simulation Techniques, SNE 4(1), 1994, and CP2 Parallel\&Distributed Simulation, SNE16(2), 2006.

These benchmarks address mainly specialists in simulation, so the educational aspects are only minor ones.

\section{Conclusion}

The inspection of the benchmarks with respect to educational aspects shows that the benchmarks are a valuable source for use in education.

For most benchmarks a calculation tool is sufficient, so that licenses for simulation systems are not necessary. Submitted and already published educational benchmark studies and educational benchmark reports show that spreadsheet tools may be an appropriate tool for working with a benchmark. And clearly, computer numeric systems as MATLAB, Octave and Python are suitable tools and suitable alternatives to special software for system simulation.

The SNE Editorial Office invites all readers and lecturers in modelling and simulation, and lecturers in applications to make use of the ARGESIM Benchmarks, and to submit a benchmark contribution describing their use of the benchmark in education. Definitions and benchmarks solutions, benchmark reports, and benchmark studies can be found at SNE's website:

\section{www.sne-journal.org/benchmarks}

Authors are asked to submit additionally the model and/or program sources used for the benchmark contribution. Only members of EUROSIM societies can download these source files, together with the high-quality colour benchmark publications.

\section{References}

[1] Breitenecker F, Körner A, Ecker H, Pawletta T. ARGESIM Benchmarks on Modelling Approaches and Simulation Implementations - Development, Classification, and Basis for Simulation Education. SNE 29(1), March 2019, to appear.

[2] Kolozsvari Z, Lienbacher L, Perebner S, Teixeira Rego H, Bicher M, Körner A, Ecker H, Breitenecker F. Time- and Event-oriented Spreadsheet Modelling of ARGESIM Benchmark C12 'Collision Processes in Rows of Spheres'. SNE 28(4), 205 - 214, 2018.

DOI: $10.11128 /$ sne.28.bne12.10452. 


\section{SNE Simulation News}

\section{EUROSIM Data and Quick Info}
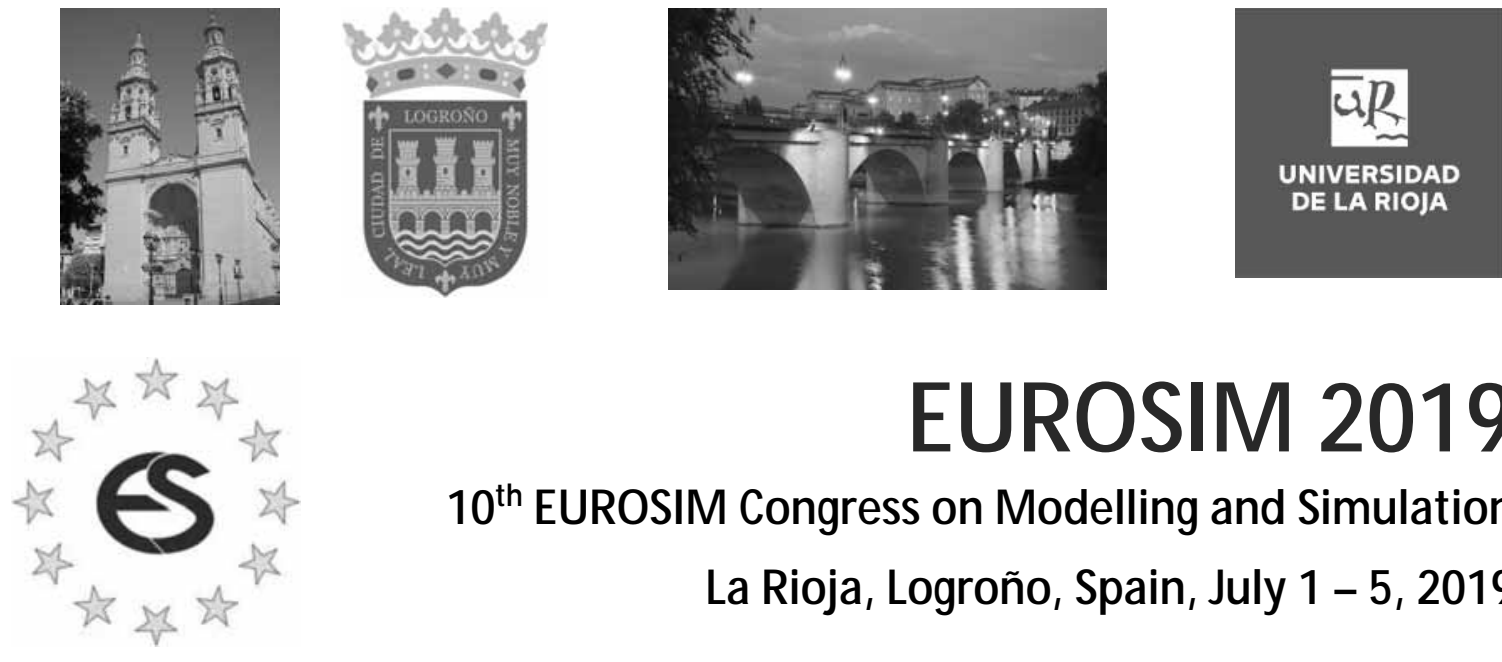

EUROSIM 2019

$10^{\text {th }}$ EUROSIM Congress on M odelling and Simulation

La Rioja, Logroño, Spain, July 1 - 5, 2019

\section{Contents}

Short Info EUROSIM .......................................... N2

Short Info ASIM , CEA-SM SG .....................................N3

Short Info CSSS, DBSS, LIOPHANT, LSS .......................N4

Short Info KA-SIM, M IM OS, NSSM, PSCS ....................N5

Short Info SIM S, SLOSIM, UKSIM ...............................N6

Short Info ROM SIM , Albanian Society ........................N7

Short Info ARGESIM , SNE .......................................N8

NewS EUROSIM ..................................................N9

News ASIM , DBSS, SLOSIM ....................................N11

News \& Call EUROSIM Congress 2019 ......................N13

Simulation Notes Europe SNE is the official membership journal of EUROSIM and distributed / available to members of the EUROSIM Societies as part of the membership benefits.

If you have any information, announcement, etc. you want to see published, please contact a member of the editorial board in your country or the editorial office. For scientific publications, please contact the EiC.

This EUROSIM Data \& Quick Info compiles data from EUROSIM societies and groups: addresses, weblinks, and officers of societies with function and email, to be published regularly in SNE issues. This information is also published at EUROSIM's website www.eurosim.info.

\section{SNE Reports Editorial Board}

EUROSIM Emilio Jiménez, emilio.jimenez@unirioja.es Andreas Körner, andreas.koerner@tuwien.ac.at Miguel Mujica Mota, m.mujica.mota@hva.nl

ASIM A. Körner, andreas.koerner@tuwien.ac.at CEA-SMSG Emilio Jiménez,emilio.jimenez@unirioja.es

CSSS Mikuláš Alexík, alexik@frtk.utc.sk

DBSS M. Mujica Mota, m.mujica.mota@hva.nl LIOPHANT F. Longo,f.longo@unical.it LSS Yuri Merkuryev, merkur@itl.rtu.lv KA-SIM Edmond Hajrizi, info@ka-sim.com MIMOS Paolo Proietti, roma@mimos.it NSSM Y. Senichenkov, senyb@dcn.icc.spbstu.ru PSCS Zenon Sosnowski, zenon@ii.pb.bialystok.pl SIMS Esko Juuso,esko.juuso@oulu.fi SLOSIMVito Logar, vito.logar@fe.uni-lj.si UKSIM A. Orsoni, A.Orsoni@kingston.ac.uk ROMSIM Marius Radulescu,mradulescu@ici.ro Albanian Society Kozeta Sevrani, kozeta.sevrani@unitir.edu.al

\section{SNE Editorial Office / ARGESIM}

$\rightarrow$ www.sne-journal.org, www.eurosim.info

麦- office@sne-journal.org,eic@sne-journal.org

$\square$ SNE Editorial Office

Johannes Tanzler (Layout, Organisation)

Irmgard Husinsky (Web, Electronic Publishing)

Felix Breitenecker EiC (Organisation, Authors)

ARGESIM/Math. Modelling \& Simulation Group,

Inst. of Analysis and Scientific Computing, TU Wien

Wiedner Hauptstrasse 8-10, 1040 Vienna, Austria 


\section{F⿻一𠃋十 EUROSIM \\ $\boldsymbol{S}$ * Federation of European Simulation Societies}

General Information. EUROSIM, the Federation of European Simulation Societies, was set up in 1989. The purpose of EUROSIM is to provide a European forum for simulation societies and groups to promote advancement of modelling and simulation in industry, research, and development. $\rightarrow$ www.eurosim.info

Member Societies. EUROSIM members may be national simulation societies and regional or international societies and groups dealing with modelling and simulation. At present EUROSIM has Full Members and $\mathrm{Ob}$ server Members, and member candidates.

\begin{tabular}{|c|c|}
\hline ASIM & $\begin{array}{l}\text { Arbeitsgemeinschaft Simulation } \\
\text { Austria, Germany, Switzerland }\end{array}$ \\
\hline CEA-SM SG & $\begin{array}{l}\text { Spanish M odelling and Simulation Group } \\
\text { Spain }\end{array}$ \\
\hline CSSS & $\begin{array}{l}\text { Czech and Slovak Simulation Society } \\
\text { Czech Republic, Slovak Republic }\end{array}$ \\
\hline DBSS & $\begin{array}{l}\text { Dutch Benelux Simulation Society } \\
\text { Belgium, Netherlands }\end{array}$ \\
\hline KA-SIM & Kosovo Simulation Society, Kosovo \\
\hline LIOPHANT & $\begin{array}{l}\text { LIOPHANT Simulation Club } \\
\text { Italy \& International }\end{array}$ \\
\hline LSS & Latvian Simulation Society; Latvia \\
\hline PSCS & $\begin{array}{l}\text { Polish Society for Computer Simulation } \\
\text { Poland }\end{array}$ \\
\hline MIMOS & $\begin{array}{l}\text { Italian M odelling and Simulation } \\
\text { Association, Italy, Observer M ember }\end{array}$ \\
\hline NSSM & $\begin{array}{l}\text { Russian National Simulation Society } \\
\text { Russian Federation }\end{array}$ \\
\hline ROM SIM & $\begin{array}{l}\text { Romanian Society for M odelling and Simu- } \\
\text { lation, Romania, Observer M ember }\end{array}$ \\
\hline SIMS & $\begin{array}{l}\text { Simulation Society of Scandinavia } \\
\text { Denmark, Finland, Norway, Sweden }\end{array}$ \\
\hline SLOSIM & $\begin{array}{l}\text { Slovenian Simulation Society } \\
\text { Slovenia }\end{array}$ \\
\hline UKSIM & $\begin{array}{l}\text { United Kingdom Simulation Society } \\
\text { UK, Ireland }\end{array}$ \\
\hline \multicolumn{2}{|c|}{ Societies in Re-Organisation: } \\
\hline CROSSIM & $\begin{array}{l}\text { Croatian Society for Simulation M odeling } \\
\text { Croatia }\end{array}$ \\
\hline FrancoSim & $\begin{array}{l}\text { Société Francophone de Simulation } \\
\text { Belgium, France }\end{array}$ \\
\hline HSS & Hungarian Simulation Society; Hungary \\
\hline ISCS & $\begin{array}{l}\text { Italian Society for Computer Simulation } \\
\text { Italy }\end{array}$ \\
\hline
\end{tabular}

EUROSIM Board / Officers. EUROSIM is governed by a board consisting of one representative of each member society, president and past president, and representatives for SNE Simulation Notes Europe. The President is nominated by the society organising the next EUROSIM Congress. Secretary, Secretary to the Board, and Treasurer are elected out of members of the board.

\begin{tabular}{ll}
\hline President & $\begin{array}{l}\text { Emilio Jiménez (CAE-SM SG), } \\
\text { emilio.jimenez@ unirioja.es }\end{array}$ \\
\hline Past President & $\begin{array}{l}\text { Esko Juuso (SIMS) } \\
\text { esko.juuso@ oulu.fi }\end{array}$ \\
\hline Secretary & $\begin{array}{l}\text { M. Mujica M ota (DBSS), } \\
\text { m.mujica.mota@ hva.nl }\end{array}$ \\
\hline Treasurer & $\begin{array}{l}\text { Felix Breitenecker (ASIM) } \\
\text { felix.breitenecker@tuwien.ac.at }\end{array}$ \\
\hline $\begin{array}{l}\text { Secretary to the } \\
\text { Board }\end{array}$ & $\begin{array}{l}\text { Andreas Körner } \\
\text { andreas.koerner@tuwien.ac.at }\end{array}$ \\
\hline Webmaster & I. Husinsky, irmgard.husinsky@tuwien.ac.at \\
\hline SNE & $\begin{array}{l}\text { Felix Breitenecker } \\
\text { Representative }\end{array}$ \\
felix.breitenecker@tuwien.ac.at
\end{tabular}

SNE - Simulation Notes Europe. SNE is a scientific journal with reviewed contributions as well as a membership newsletter for EUROSIM with information from the societies in the News Section. EUROSIM societies are offered to distribute to their members the journal SNE as official membership journal. SNE Publishers are EUROSIM, ARGESIM and ASIM.

\begin{tabular}{ll}
\hline SNE & Felix Breitenecker \\
Editor-in-Chief & felix.breitenecker@tuwien.ac.at \\
\hline$\rightarrow$ www.sne-journal.org, \\
\hline 莑: office@sne-journal.org
\end{tabular}

EUROSIM Congress. EUROSIM is running the triennial conference series EUROSIM Congress. The congress is organised by one of the EUROSIM societies.

EUROSIM 2019, the 10th EUROSIM Congress, will be organised by CEA-SM SG, the Spanish Simulation Society, in La Rioja, Logroño, Spain, July 1 - 5, 2019.

\begin{tabular}{l}
\hline Chairs / Team EUROSIM 2019 \\
\hline $\begin{array}{r}\text { Emilio Jiménez, EUROSIM President, } \\
\text { emilio.jimenez@unirioja.es }\end{array}$ \\
Juan Ignacio Latorre, juanignacio.latorre@unavarra.es \\
\hline www.eurosim.info
\end{tabular}




\section{EUROSIM Member Societies}

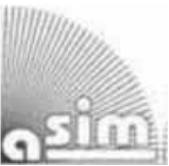

\section{ASIM \\ German Simulation Society \\ Arbeitsgemeinschaft Simulation}

ASIM (Arbeitsgemeinschaft Simulation) is the association for simulation in the German speaking area, servicing mainly Germany, Switzerland and Austria. ASIM was founded in 1981 and has now about 400 individual members (including associated), and 90 institutional or industrial members.

\section{$\rightarrow$ www.asim-gi.org with members' area \\ 暳”info@asim-gi.org,admin@asim-gi.org \\ $\triangle$ ASIM - Inst. of Analysis and Scientific Computing \\ Vienna University of Technology (TU Wien) \\ Wiedner Hauptstraße 8-10, 1040 Vienna, Austria}

\begin{tabular}{|c|c|}
\hline ASIM Officers & \\
\hline President & $\begin{array}{l}\text { Felix Breitenecker } \\
\text { felix.breitenecker@tuwien.ac.at }\end{array}$ \\
\hline \multirow[t]{3}{*}{ Vice presidents } & Sigrid Wenzel, s.wenzel@uni-kassel.de \\
\hline & T. Pawletta, thorsten.pawletta@hs-wismar.de \\
\hline & A. Körner, andreas.koerner@tuwien.ac.at \\
\hline \multirow[t]{2}{*}{ Secretary } & Ch. Deatcu, christina.deatcu@hs-wismar.de \\
\hline & I. Husinsky, Irmgard.husinsky@tuwien.ac.at \\
\hline Treasurer & Anna M athe, anna.mathe@tuwien.ac.at \\
\hline \multirow{3}{*}{$\begin{array}{l}\text { M embership } \\
\text { Affairs }\end{array}$} & S.Wenzel, s.wenzel@uni-kassel.de \\
\hline & Ch. Deatcu, christina.deatcu@ @s-wismar.de \\
\hline & F. Breitenecker, felix.breitenecker@tuwien.ac.at \\
\hline \multirow[t]{2}{*}{ Repr. EUROSIM } & F. Breitenecker, felix.breitenecker@tuwien.ac.at \\
\hline & A. Körner, andreas.koerner@tuwien.ac.at \\
\hline \multirow{2}{*}{$\begin{array}{l}\text { Intemat. Affairs } \\
\text { - GI Contact }\end{array}$} & O. Rose, Oliver.Rose@tu-dresden.de \\
\hline & N. Popper, niki.popper@dwh.at \\
\hline \multirow{2}{*}{$\begin{array}{l}\text { Editorial Board } \\
\text { SNE }\end{array}$} & T. Pawletta, thorsten.pawletta@hs-wismar.de \\
\hline & Ch. Deatcu, christina.deatcu@ @s-wismar.de \\
\hline Web EuroSim & I. Husinsky, Irmgard.husinsky@tuwien.ac.at \\
\hline
\end{tabular}

ASIM is organising / co-organising the following international conferences:

- ASIM Int. Conference 'Simulation in Production and Logistics' - biannual

- ASIM 'Symposium Simulation Technique' - biannual

- MATHM OD Int. Vienna Conference on

Mathmatical Modelling - triennial

Furthermore, ASIM is co-sponsor of WSC - Winter Simulation Conference, of SCS conferences SpringSim and SummerSim, and of I3M and Simutech conference series.

\begin{tabular}{|c|c|}
\hline \multicolumn{2}{|c|}{ ASIM Working Committees } \\
\hline GMMS & $\begin{array}{l}\text { Methods in Modelling and Simulation } \\
\text { Th. Pawletta, thorsten.pawletta@hs-wismar.de }\end{array}$ \\
\hline SUG & $\begin{array}{l}\text { Simulation in Environmental Systems } \\
\text { Jochen Wittmann, } \\
\text { wittmann@ informatik.uni-hamburg.de }\end{array}$ \\
\hline STS & $\begin{array}{l}\text { Simulation of Technical Systems } \\
\text { Walter Commerell, commerell@ hs-ulm.de }\end{array}$ \\
\hline SPL & $\begin{array}{l}\text { Simulation in Production and Logistics } \\
\text { Sigrid Wenzel, s.wenzel@ uni-kassel.de }\end{array}$ \\
\hline EDU & $\begin{array}{l}\text { Simulation in Education/Education in Simulation } \\
\text { A. Körner, andreas.koerner@tuwien.ac.at }\end{array}$ \\
\hline $\begin{array}{l}\text { BIG } \\
\text { DATA }\end{array}$ & $\begin{array}{l}\text { Working Group Data-driven Simulation in Life } \\
\text { Sciences; niki.popper@dwh.at }\end{array}$ \\
\hline $\begin{array}{l}\text { WORKING } \\
\text { GROUPS }\end{array}$ & $\begin{array}{l}\text { Simulation in Business Administration, in Traffic } \\
\text { Systems, for Standardisation, etc. }\end{array}$ \\
\hline
\end{tabular}

\section{CEA-SM SG - Spanish Modelling and Simulation Group}

CEA is the Spanish Society on Automation and Control and it is the national member of IFAC (International Federation of Automatic Control) in Spain. Since 1968 CEAIFAC looks after the development of the Automation in Spain, in its different issues: automatic control, robotics, SIMULATION, etc. The association is divided into national thematic groups, one of which is centered on Modeling, Simulation and Optimization, constituting the CEA Spanish Modeling and Simulation Group (CEA-SM SG). It looks after the development of the Modelling and Simulation (M\&S) in Spain, working basically on all the issues concerning the use of $\mathrm{M} \& \mathrm{~S}$ techniques as essential engineering tools for decision-making and optimization.

$\rightarrow$ http://www.ceautomatica.es/grupos/

$\rightarrow$ emilio.jimenez@unirioja.es

simulacion@cea-ifac.es

$\triangle$ CEA-SMSG / Emilio Jiménez, Department of Electrical Engineering, University of La Rioja, San José de Calasanz 31, 26004 Logroño (La Rioja), SPAIN

\begin{tabular}{ll}
\hline CEA - SM SG Officers \\
\hline President & $\begin{array}{l}\text { Emilio Jiménez, } \\
\text { emilio.jimenez@unirioja.es }\end{array}$ \\
\hline Vice president & $\begin{array}{l}\text { Juan Ignacio Latorre, } \\
\text { juanignacio.latorre@unavarra.es }\end{array}$ \\
\hline Repr. EUROSIM & Emilio Jiménez, emilio.jimenez@unirioja.es \\
\hline Edit. Board SNE & Juan Ignacio Latorre, \\
& juanignacio.latorre@unavarra.es \\
\hline Web EUROSıM & Mercedes Perez mercedes.perez@unirioja.es
\end{tabular}

Last data update February 2018 


\section{CSSS - Czech and Slovak csss Simulation Society}

CSSS -The Czech and Slovak Simulation Society has about 150 members working in Czech and Slovak national scientific and technical societies (Czech Society for Applied Cybernetics and Informatics, Slovak Society for Applied Cybernetics and Informatics). CSSS main objectives are: development of education and training in the field of modelling and simulation, organising professional workshops and conferences, disseminating information about modelling and simulation activities in Europe. Since 1992, CSSS is full member of EUROSIM.

$\rightarrow$ www.fit.vutbr.cz/CSSS

奉”” snorek@fel.cvut.cz

$\triangle$ CSSS / Miroslav Šnorek, CTU Prague

FEE, Dept. Computer Science and Engineering,

Karlovo nam. 13, 12135 Praha 2, Czech Republic

\begin{tabular}{ll}
\hline CSSS Officers & \\
\hline President & Miroslav Šnorek, snorek@fel.cvut.cz \\
\hline Vice president & M ikuláš Alexík, alexik@ frtk.fri.utc.sk \\
\hline Scientific Secr. & A. Kavička, Antonin.Kavicka@ upce.cz \\
\hline Repr. EuRoSım & M iroslav Šnorek, snorek@ fel.cvut.cz \\
\hline Edit. Board SNE & M ikuláš Alexík, alexik@ frtk.fri.utc.sk \\
\hline Web EuRoSım & Petr Peringer, peringer@ fit.vutbr.cz \\
\hline
\end{tabular}

\section{DBSS - Dutch Benelux Simulation Society}

The Dutch Benelux Simulation Society (DBSS) was founded in July 1986 in order to create an organisation of simulation professionals within the Dutch language area. DBSS has actively promoted creation of similar organisations in other language areas. DBSS is a member of EUROSIM and works in close cooperation with its members and with affiliated societies.

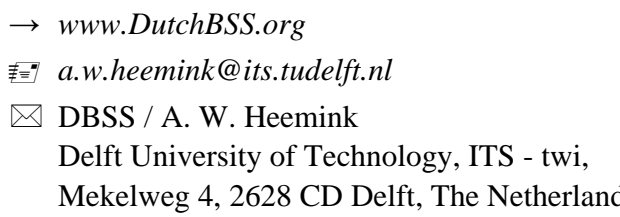

\begin{tabular}{ll}
\hline DBSS Officers & \\
\hline President & M. Mujica M ota, m.mujica.mota@ hva.nl \\
\hline Vice president & A. Heemink, a.w.heemink@ its.tudelft.nl \\
\hline Treasurer & A. Heemink, a.w.heemink@ its.tudelft.nl \\
\hline Secretary & P. M. Scala, p.m.scala@ hva.nl \\
\hline Repr. EuRoSim & M. Mujica M ota, m.mujica.mota@ hva.nl \\
\hline Edit. SNE/Web & M. Mujica M ota, m.mujica.mota@ hva.nl \\
\hline
\end{tabular}
Last data update June 2016

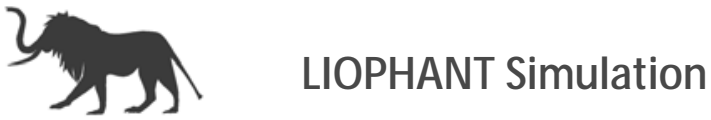

Liophant Simulation is a non-profit association born in order to be a trait-d'union among simulation developers and users; Liophant is devoted to promote and diffuse the simulation techniques and methodologies; the Association promotes exchange of students, sabbatical years, organization of International Conferences, courses and internships focused on $\mathrm{M} \& \mathrm{~S}$ applications.

$\rightarrow$ www.liophant.org

麦”; info@liophant.org

$\triangle$ LIOPHANT Simulation, c/o Agostino G. Bruzzone, DIME, University of Genoa, Savona Campus via Molinero 1, 17100 Savona (SV), Italy

\begin{tabular}{ll}
\hline \multicolumn{2}{l}{ LIOPHANT Officers } \\
\hline President & A.G. Bruzzone, agostino@ itim.unige.it \\
\hline Director & E. Bocca, enrico.bocca@ liophant.org \\
\hline Secretary & A. Devoti, devoti.a@ iveco.com \\
\hline Treasurer & M arina M assei, massei@ itim.unige.it \\
\hline Repr. EuroSIM & A.G. Bruzzone, agostino@ itim.unige.it \\
\hline Deputy & F. Longo, f.longo@ unical.it \\
\hline Edit. Board SNE & F. Longo, f.longo@ unical.it \\
\hline Web EuRoSIM & F. Longo, f.longo@ unical.it \\
\hline
\end{tabular}

\section{LSS - Latvian Simulation Society}

The Latvian Simulation Society (LSS) has been founded in 1990 as the first professional simulation organisation in the field of Modelling and simulation in the post-Soviet area. Its members represent the main simulation centres in Latvia, including both academic and industrial sectors.

\section{$\rightarrow$ briedis.itl.rtu.lv/imb/ \\ 夆=7merkur@itl.rtu.lv \\ $\triangle$ LSS / Yuri Merkuryev, Dept. of Modelling and Simulation Riga Technical University Kalku street 1, Riga, LV-1658, LATVIA}

\begin{tabular}{ll}
\hline LSS Officers & \\
\hline President & Yuri M erkuryev, merkur@itl.rtu.Iv \\
\hline Secretary & Artis Teilans, Artis.Teilans@ exigenservices.com \\
\hline Repr. EuROSIM & Yuri M erkuryev, merkur@itl.rtu.Iv \\
\hline Deputy & Artis Teilans, Artis.Teilans@ exigenservices.com \\
\hline Edit. Board SNE & Yuri M erkuryev, merkur@ itl.rtu.Iv \\
\hline Web EuROSIm & Vitaly Bolshakov, vitalijs.bolsakovs@ rtu.lv \\
\hline
\end{tabular}




\section{KA-SIM Kosovo Simulation Society}

Kosova Association for Modeling and Simulation (KASIM, founded in 2009), is part of Kosova Association of Control, Automation and Systems Engineering (KACASE). KA-CASE was registered in 2006 as non Profit Organization and since 2009 is National Member of IFAC - International Federation of Automatic Control. KA-SIM joined EUROSIM as Observer Member in 2011. In 2016, KA-SIM became full member.

KA-SIM has about 50 members, and is organizing the international conference series International Conference in Business, Technology and Innovation, in November, in Durrhes, Albania, and IFAC Simulation Workshops in Pristina.

$\rightarrow$ www.ubt-uni.net/ka-case

拝”- ehajrizi@ubt-uni.net

$\checkmark$ MOD\&SIM KA-CASE; Att. Dr. Edmond Hajrizi

Univ. for Business and Technology (UBT)

Lagjja Kalabria p.n., 10000 Prishtina, Kosovo

\begin{tabular}{ll}
\hline KA-SIM Officers & \\
\hline President & Edmond Hajrizi, ehajrizi@ ubt-uni.net \\
\hline Vice president & M uzafer Shala, info@ ka-sim.com \\
\hline Secretary & Lulzim Beqiri, info@ ka-sim.com \\
\hline Treasurer & Selman Berisha, info@ ka-sim.com \\
\hline Repr. EuRoSIm & Edmond Hajrizi, ehajrizi@ ubt-uni.net \\
\hline Deputy & M uzafer Shala, info@ ka-sim.com \\
\hline Edit. Board SNE & Edmond Hajrizi, ehajrizi@ ubt-uni.net \\
\hline Web EuRoSIM & Betim Gashi, info@ ka-sim.com \\
\hline
\end{tabular}

\section{MIMOS - Italian Modelling and Simulation Association}

MIMOS (Movimento Italiano Modellazione e Simulazione - Italian Modelling and Simulation Association) is the Italian association grouping companies, professionals, universities, and research institutions working in the field of modelling, simulation, virtual reality and 3D, with the aim of enhancing the culture of 'virtuality' in Italy, in every application area.

MIM OS became EUROSIM Observer Member in 2016 and EUROSIM Full Member in September 2018.

$\rightarrow$ www.mimos.it

麦引roma@mimos.it-info@mimos.it

$\triangle$ MIMOS - Movimento Italiano Modellazione e Simulazione; via Ugo Foscolo 4, 10126 Torino via Laurentina 760, 00143 Roma

\begin{tabular}{ll}
\hline MIM 0S Officers & \\
\hline President & Paolo Proietti, roma@mimos.it \\
\hline Secretary & Davide Borra, segreteria@ mimos.it \\
\hline Treasurer & Davide Borra, segreteria@mimos.it \\
\hline Repr. EuRoSım & Paolo Proietti, roma@ mimos.it \\
\hline Deputy & $\begin{array}{l}\text { Agostino Bruzzone, } \\
\text { agostino@ itim.unige.it }\end{array}$ \\
\hline Edit. Board SNE & Paolo Proietti, roma@ mimos.it \\
\hline & \multicolumn{1}{c}{ Last data update December 2016 }
\end{tabular}

\section{NSSM - National Society for Simulation Modelling (Russia)}

NSSM - The Russian National Simulation Society (Национальное Общество Имитационного Моделирования - НОИМ) was officially registered in Russian Federation on February 11, 2011. In February 2012 NSS has been accepted as an observer member of EUROSIM, and in 2015 NSSM has become full member.

$\rightarrow$ www.simulation.su
莑 yusupov@iias.spb.su
$\square$ NSSM / R. M. Yusupov,

St. Petersburg Institute of Informatics and Automation RAS, 199178, St. Petersburg, 14th lin. V.O, 39

\begin{tabular}{ll}
\hline NSSM Officers & \\
\hline President & R. M. Yusupov, yusupov@iias.spb.su \\
\hline Chair Man. Board & A. Plotnikov, plotnikov@sstc.spb.ru \\
\hline Secretary & M. Dolmatov, dolmatov@simulation.su \\
\hline Repr. EuRoSim & $\begin{array}{l}\text { R.M. Yusupov, yusupov@iias.spb.su } \\
\text { Y. Senichenkov, } \\
\text { senyb@dcn.icc.spbstu.ru }\end{array}$ \\
\hline Deputy & B. Sokolov, sokol@iias.spb.su \\
\hline Edit. Board SNE & $\begin{array}{c}\text { Y. Senichenkov, senyb@ mail.ru, } \\
\text { senyb@dcn.icc.spbstu.ru, }\end{array}$ \\
\hline & $\quad$ Last data update February 2018
\end{tabular}

\section{PSCS - Polish Society for Computer Simulation}

PSCS was founded in 1993 in Warsaw. PSCS is a scientific, non-profit association of members from universities, research institutes and industry in Poland with common interests in variety of methods of computer simulations and its applications. At present PSCS counts 257 members. 
$\rightarrow$ www.eurosim.info, www.ptsk.pl/

麦”leon@ibib.waw.pl

$\triangle$ PSCS / Leon Bobrowski, c/o IBIB PAN, ul. Trojdena 4 (p.416), 02-109 Warszawa, Poland

\begin{tabular}{ll}
\hline PSCS Officers & \\
\hline President & Leon Bobrowski, leon@ibib.waw.pl \\
\hline Vice president & Tadeusz Nowicki, \\
& Tadeusz.Nowicki@ wat.edu.pl \\
\hline Treasurer & Z. Sosnowski, zenon@ii.pb.bialystok.pl \\
\hline Secretary & Zdzislaw Galkowski, \\
& Zdzislaw.Galkowski@ simr.pw.edu.pl \\
\hline Repr. EuroSim & Leon Bobrowski, leon@ ibib.waw.pl \\
\hline Deputy & Tadeusz Nowicki, tadeusz.nowicki@wat.edu.pl \\
\hline Edit. Board SNE & Zenon Sosnowski, z.sosnowski@pb.ed.pl \\
\hline Web EuroSım & Magdalena Topczewska \\
& m.topczewska@ pb.edu.pl \\
\hline
\end{tabular}

\section{SIMS - Scandinavian Simulation Society}

SIMS is the Scandinavian Simulation Society with members from the five Nordic countries Denmark, Finland, Iceland, Norway and Sweden. The SIMS history goes back to 1959. SIMS practical matters are taken care of by the SIMS board consisting of two representatives from each Nordic country (Iceland one board member).

SIMS Structure. SIMS is organised as federation of regional societies. There are FinSim (Finnish Simulation Forum), MoSis (Society for Modelling and Simulation in Sweden), DKSIM (Dansk Simuleringsforening) and NFA (Norsk Forening for Automatisering).

$\rightarrow \quad$ www.scansims.org

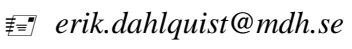

$\triangle$ SIMS / Erik Dahlquist, School of Business, Society and Engineering, Department of Energy, Building and Environment, Mälardalen University, P.O.Box 883, 72123

Västerås, Sweden

\begin{tabular}{ll}
\hline SIMS Officers & \\
\hline President & Erik Dahlquist, erik.dahlquist@ mdh.se \\
\hline Vice president & Bernt Lie, Bernt.Lie@ @ usn.no \\
\hline Treasurer & $\begin{array}{l}\text { Vadim Engelson, } \\
\text { vadim.engelson@ mathcore.com }\end{array}$ \\
\hline Repr. EuRoSım & Erik Dahlquist, erik.dahlquist@ mdh.se \\
\hline Edit. Board SNE & Esko Juuso, esko.juuso@oulu.fi \\
\hline Web EuRoSim & Vadim Engelson, \\
& vadim.engelson@ mathcore.com \\
\hline
\end{tabular}

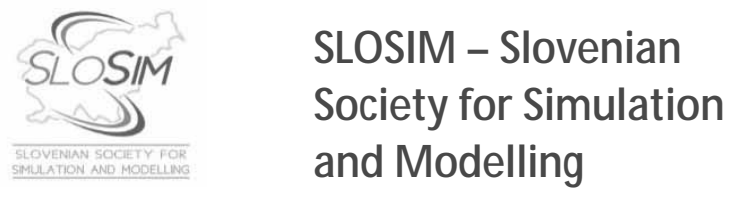

SLOSIM - Slovenian Society for Simulation and Modelling was established in 1994 and became the full member of EUROSIM in 1996. Currently it has 90 members from both Slovenian universities, institutes, and industry. It promotes modelling and simulation approaches to problem solving in industrial as well as in academic environments by establishing communication and cooperation among corresponding teams.

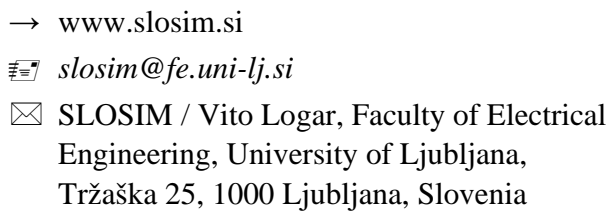

\begin{tabular}{ll}
\hline \multicolumn{2}{l}{ SLOSIM Officers } \\
\hline President & Vito Logar, vito.logar@fe.uni-lj.si \\
\hline Vice president & Božidar Šarler, bozidar.sarler@ung.si \\
\hline Secretary & Simon Tomažič, simon.tomazic@fe.uni-lj.si \\
\hline Treasurer & Milan Simčič, milan.simcic@fe.uni-lj.si \\
\hline Repr. EURoSIm & B.Zupančič, borut.zupancic@fe.uni-lj.si \\
\hline Deputy & Vito Logar, vito.logar@fe.uni-lj.si \\
\hline Edit. Board SNE & R. Karba, rihard.karba@fe.uni-lj.si \\
\hline Web EuRoSIM & Vito Logar, vito.logar@fe.uni-lj.si \\
\hline
\end{tabular}

\section{UKSIM - United Kingdom Simulation Society}

The UK Simulation Society is very active in organizing conferences, meetings and workshops. UKSim holds its annual conference in the March-April period. In recent years the conference has always been held at Emmanuel College, Cambridge. The Asia Modelling and Simulation Section (AMSS) of UKSim holds 4-5 conferences per year including the EMS (European Modelling Symposium), an event mainly aimed at young researchers, organized each year by UKSim in different European cities. Membership of the UK Simulation Society is free to participants of any of our conferences and their co-authors.

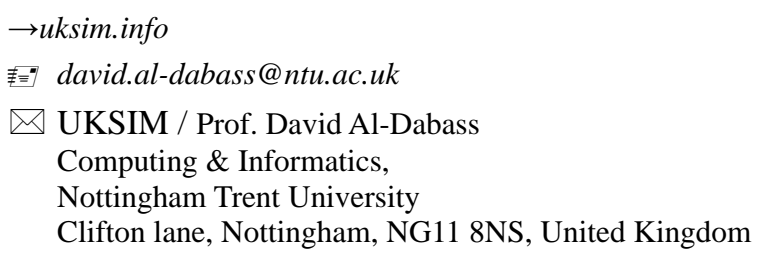




\begin{tabular}{ll}
\hline UKSIM Officers & \\
\hline President & $\begin{array}{l}\text { David Al-Dabass, } \\
\text { david.al-dabass@ ntu.ac.uk }\end{array}$ \\
\hline Secretary & A. Orsoni, A.Orsoni@kingston.ac.uk \\
\hline Treasurer & A. Orsoni, A.Orsoni@ kingston.ac.uk \\
\hline $\begin{array}{l}\text { Membership } \\
\text { chair }\end{array}$ & G. Jenkins, glenn.l.jenkins@smu.ac.uk \\
\hline Local/Venue chair & Richard Cant, richard.cant@ntu.ac.uk \\
\hline Repr. EuRoSIM & A. Orsoni, A.Orsoni@ kingston.ac.uk \\
\hline Deputy & G. Jenkins, glenn.l.jenkins@smu.ac.uk \\
\hline Edit. Board SNE & A. Orsoni, A.Orsoni@ kingston.ac.uk \\
\hline
\end{tabular}

\section{EUROSIM Observer Members}

\section{ROM SIM - Romanian Modelling and Simulation Society}

ROMSIM has been founded in 1990 as a non-profit society, devoted to theoretical and applied aspects of modelling and simulation of systems. ROMSIM currently has about 100 members from Romania and Moldavia.

$\rightarrow$ www.eurosim.info (www.ici.ro/romsim)

莑 sflorin@ici.ro

$\triangle$ ROMSIM / Florin Hartescu,

National Institute for Research in Informatics, Averescu Av. 8 - 10, 71316 Bucharest, Romania

\begin{tabular}{lr}
\hline ROM SIM Officers \\
\hline $\begin{array}{l}\text { President } \\
\text { Vice president }\end{array}$ & $\begin{array}{r}\text { Florin Hartescu, flory@ ici.ro } \\
\text { Marius Radulescu, } \\
\text { mradulescu.csmro@yahoo.com }\end{array}$ \\
\hline Repr. EUROSIM & $\begin{array}{r}\text { Marius Radulescu, } \\
\text { mradulescu.csmro@yahoo.com }\end{array}$ \\
\hline Deputy & Florin Hartescu, flory@ici.ro \\
\hline Edit. Board SNE & Constanta Zoe Radulescu, zoe@ ici.ro \\
\hline Web EuRoSIM & Florin Hartescu, flory@ici.ro \\
\hline
\end{tabular}

\section{Member CANDIDATES}

\section{Albanian SIMULATION Society}

At the Department of Statistics and Applied Informatics, Faculty of Economy, University of Tirana, Prof. Dr. Kozeta Sevrani at present is setting up an Albanian Simulation Society. Kozeta Sevrani, professor of Computer Science and Management Information Systems, and head of the Department of Mathematics, Statistics and Applied Informatic, has attended the a EUROSIM board meeting in Vienna Feb. 2016.
There she has presented simulation activities in Albania and the new simulation society.

The society - constitution and bylaws are being worked out - will be involved in different international and local simulation projects, and will be engaged in the organisation of the conference series ISTI - Information Systems and Technology. The society intends to become a EUROSIM Observer Member.

拝三” kozeta.sevrani@unitir.edu.al

$\triangle$ Albanian Simulation Goup, attn. Kozeta Sevrani

University of Tirana, Faculty of Economy

rr. Elbasanit, Tirana 355 Albania

\begin{tabular}{lc}
\hline Albanian Simulation Society- Officers (Planned) \\
\hline President & $\begin{array}{c}\text { Kozeta Sevrani, } \\
\text { kozeta.sevrani@ unitir.edu.al }\end{array}$ \\
\hline Repr. EuroSim & $\begin{array}{c}\text { Kozeta Sevrani, } \\
\text { kozeta.sevrani@ unitir.edu.al }\end{array}$ \\
\hline Edit. Board SNE & $\begin{array}{c}\text { Albana Gorishti, } \\
\text { albana.gorishti@ unitir.edu.al } \\
\text { Majlinda Godolja, } \\
\text { majlinda.godolja@fshn.edu.al }\end{array}$ \\
\hline
\end{tabular}

\section{Societies in Re-Organisation}

The following societies are at present inactive or under re-organisation:

- CROSSIM

Croatian Society for Simulation Modelling

- FRANCOSIM - Société Francophone de Simulation

- HSS - Hungarian Simulation Society

- ISCS - Italian Society for Computer Simulation 


\section{Association Simulation News}

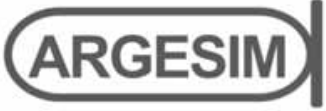

ARGESIM is a non-profit association generally aiming for dissemination of information on system simulation from research via development to applications of system simulation. ARGESIM is closely co-operating with EUROSIM, the Federation of European Simulation Societies, and with ASIM, the German Simulation Society. ARGESIM is an 'outsourced' activity from the Mathematical Modelling and Simulation Group of TU Wien, there is also close co-operation with TU Wien (organisationally and personally).

$\rightarrow$ www.argesim.org

麦=" $\rightarrow$ office@argesim.org

$\bowtie \rightarrow$ ARGESIM/Math. Modelling \& Simulation Group, Inst. of Analysis and Scientific Computing, TU Wien Wiedner Hauptstrasse 8-10, 1040 Vienna, Austria Attn. Prof. Dr. Felix Breitenecker

ARGESIM is following its aims and scope by the following activities and projects:

- Publication of the scientific journal SNE Simulation Notes Europe (membership journal of EUROSIM, the Federation of European Simulation Societies) - www.sne-journal.org

- Organisation and Publication of the ARGESIM Benchmarks for Modelling Approaches and Simulation Implementations

- $\quad$ Publication of the series ARGESIM Reports for monographs in system simulation, and proceedings of simulation conferences and workshops

- Publication of the special series FBSSimulation Advances in Simulation / Fortschrittsberichte Simulation - monographs in co-operation with ASIM, the German Simulation Society

- Organisation of the Conference Series MATHMOD Vienna (triennial, in co-operation with EUROSIM, ASIM, and TU Wien) - www.mathmod.at

- Organisation of Seminars and Summerschools on Simulation

- Administration of ASIM (German Simulation Society) and administrative support for EUROSIM www.eurosim.info

- Support of ERASMUS and CEEPUS activities in system simulation for TU Wien

ARGESIM is a registered non-profit association and a registered publisher: ARGESIM Publisher Vienna, root ISBN 978-3-901608-xx-y, root DOI 10.11128/z...zz.zz. Publication is open for ASIM and for EUROSIM Member Societies.

\section{SNE - Simulation Notes Europe}

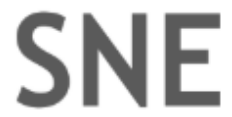

The scientific journal SNE - Simulation Notes Europe provides an international, high-quality forum for presentation of new ideas and approaches in simulation - from modelling to experiment analysis, from implementation to verification, from validation to identification, from numerics to visualisation - in context of the simulation process. SNE puts special emphasis on the overall view in simulation, and on comparative investigations.

Furthermore, SNE welcomes contributions on education in/for/with simulation.

SNE is also the forum for the ARGESIM Benchmarks on Modelling Approaches and Simulation Implementations publishing benchmarks definitions, solutions, reports and studies - including model sources via web.

$\rightarrow$ www.sne-journal.org,

莑=゙ $\rightarrow$ office@sne-journal.org,eic@sne-journal.org

$\bowtie \rightarrow$ SNE Editorial Office

ARGESIM/Math. Modelling \& Simulation Group, Inst. of Analysis and Scientific Computing, TU Wien Wiedner Hauptstrasse 8-10, 1040 Vienna, Austria EiC Prof. Dr. Felix Breitenecker

SNE, primarily an electronic journal, follows an open access strategy, with free download in basic layout. SNE is the official membership journal of EUROSIM, the Federation of European Simulation Societies. Members of EUROSIM Societies are entitled to download SNE in highquality, and to access additional sources of benchmark publications, model sources, etc. On the other hand, SNE offers EUROSIM Societies a publication forum for postconference publication of the society's international conferences, and the possibility to compile thematic or event-based SNE Special Issues.

Simulationists are invited to submit contributions of any type - Technical Note, Short Note, Project Note, Educational Note, Benchmark Note, etc. via SNE's website:

\section{CNE SIMULATION NOTES EUROPE}

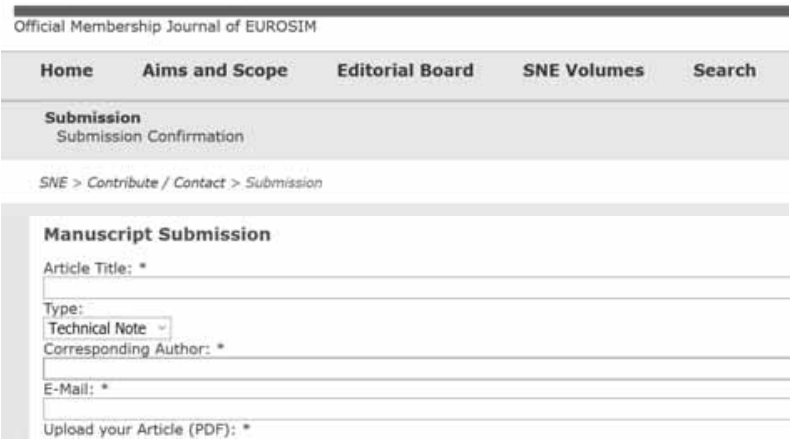


EUROSIM

\section{Federation of European Simulation Societies}

EUROSIM, the Federation of European Simulation Societies, was set up in 1989. The purpose of EUROSIM is to provide a European forum for simulation societies and groups to promote advancement of modelling and simulation in industry, research, and development.

\section{Member Societies / Member Benefits}

EUROSIM members may be national simulation societies and regional or international societies and groups dealing with modelling and simulation. The following snapshot of the societies' page at the EUROSIM website www.eurosim.info lists the active member societies.

\section{$\Leftrightarrow$ EUROSIM}

Federation of European Simulation Societies

EUROSIM News Events Societies Jou
Member Societies
Full Members
- ASIM - Arbeitsgemeinschaft Simulation (Austria, Germany,
- CEA SMSG - Spanish Modelling and Simulation Group
- DSS- Czech and Slovak Simulation Society
- KA-SIM - Kosova Society for Modeling and Simulation
- LIOPHANT SIMULATION - International Modelling and Sirm
- LSS- Latvian Society for Simulation
- MIMOS- Italian Modelling and Simulation Association
- PSCS- National Society for Simulation Modelling in Russia
- SIMS- Simulation Society for Computer Simulation
- SLOSIM- Slovenian Society for Simulation and Modelling
- UKSIM- United Kingdom Simulation Society (UK, Ireland)
Observer Members

- ROMSIM- Romanian Society for Modelling and Simulation

EUROSIM offers various benefits for the personal and institutional members of the EUROSIM Member Societies, e.g. reduced conference fees, access to and publication in EUROSIM's scientific journal SNE Simulation Notes Europe, services via EUROSIM's website (publication access to SNE and to proceedings of conferences, etc.

\section{EUROSIM Congress - Conferences - Events}

EUROSIM is organising, supporting, or co-sponsoring resp. i) the triennial EUROSIM Congress, ii) EUROSIM Conferences and EUROSIM Events-e.g. the MATHMOD Conference Series or international conferences of the EUROSIM Member Societies, iii) international conferences on basis of mutual co-sponsorship, e.g. I3M Conference Series.
The EUROSIM Congress can be seen as constant within these developments - every three years simulationists from all over the world gather in one European country to exchange information on development in modelling and simulation.

The $10^{\text {th }}$ EUROSIM Congress will take place in July 2019 in Logroño, La Rioja, Spain. The First Call and further details on the congress can be found on the congress website www.eurosim2019.com at the end of this News Section.

\section{EUROSIM 2019}

$10^{\text {th }}$ EUROSIM Congress on M odelling and Simulation

\section{July 2019, La Rioja, Logroño, Spain} www.eurosim2019.com

\section{EUROSIM Website / New Publications / Login} The EUROSIM website www.eurosim.info informs on EUROSIM and its societies and publications.

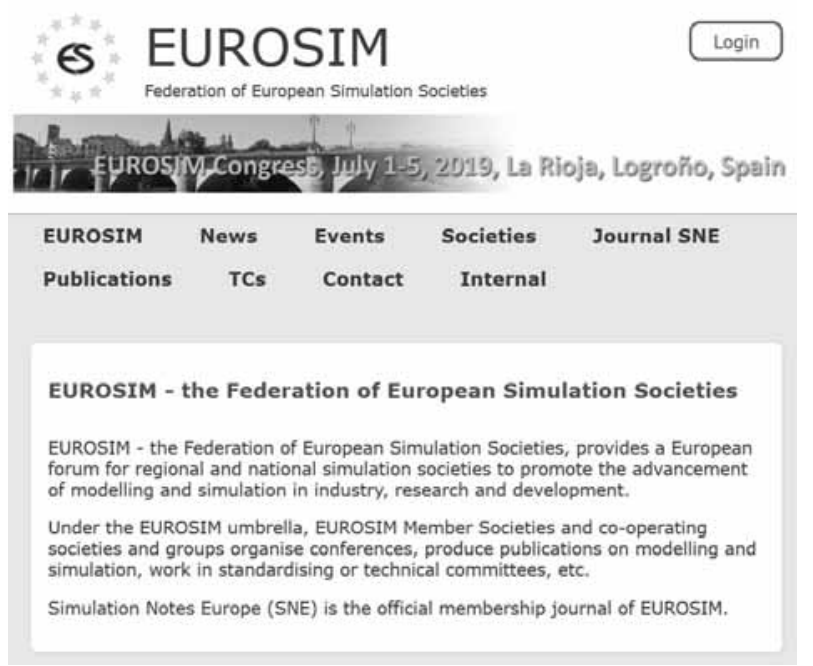

The News section and the Events section are maintained by the EUROSIM webmaster(s) compiling classical news lists and events lists. Please send an e-mail to webmaster@eurosim.info if you have an announcement to be posted (general news, general news about a society, conference announcements).

The Societies section -webpage provides information on all EUROSIM Member Societies - the content can be managed by the societies themselves. 
The Journal SNE section shortly introduces SNE Simulation Notes Europe as official membership journal of EUROSIM and links to the SNE website. The Publications section is intended to provide Proceedings from EUROSIM Congresses and from EUROSIM Conferences (e.g. MATHM OD, ICBTI), with Login for members of EUROSIM societies.

Access for members of EUROSIM Societies. Highquality colour Online SNE, full SNE Archive, and sources of benchmarks or other additional documents are available for members of EUROSIM societies. Login data are distributed by the EUROSIM societies to their members directly:

- (Sample) Login: asim-member

- Password: $\mathbf{x x x x \times x}$

This is also the login for the SNE website (for SNE download), as well as for the EUROSIM website (for publication download, see below) - please use Login button, or enter Login section resp.

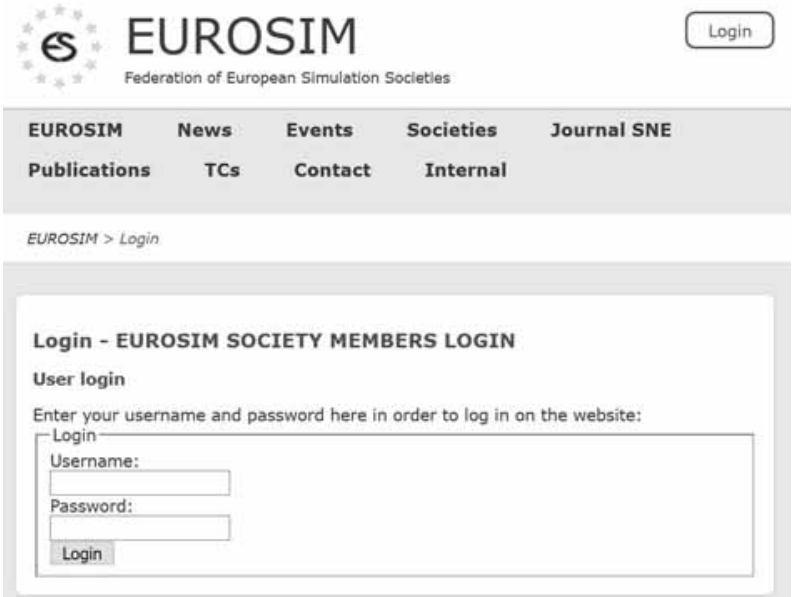

Proceedings and Special Issues. This year Extended Proceedings of the EUROSIM Congress Oulu have been published. The short contributions are available in SNE 28(3) as special issue Short Papers 9th EUROSIM Congress, Oulu, 2016 with access to the high-quality version for members of EUROSIM Societies (website snapshot at right). Furthermore, the EUROSIM website also provides publications from EUROSIM Conferences - in 2018 also the MATHMOD 2018 Extended Abstract Volume (short contributions to MATHMOD 2018 Vienna Conference) have been published by ARGESIM Publisher, and are available at the EUROSIM website, with access to the high-quality version for members of EUROSIM Societies (website snapshot at right).

\section{CNE SIMULATION NOTES EUROPE}

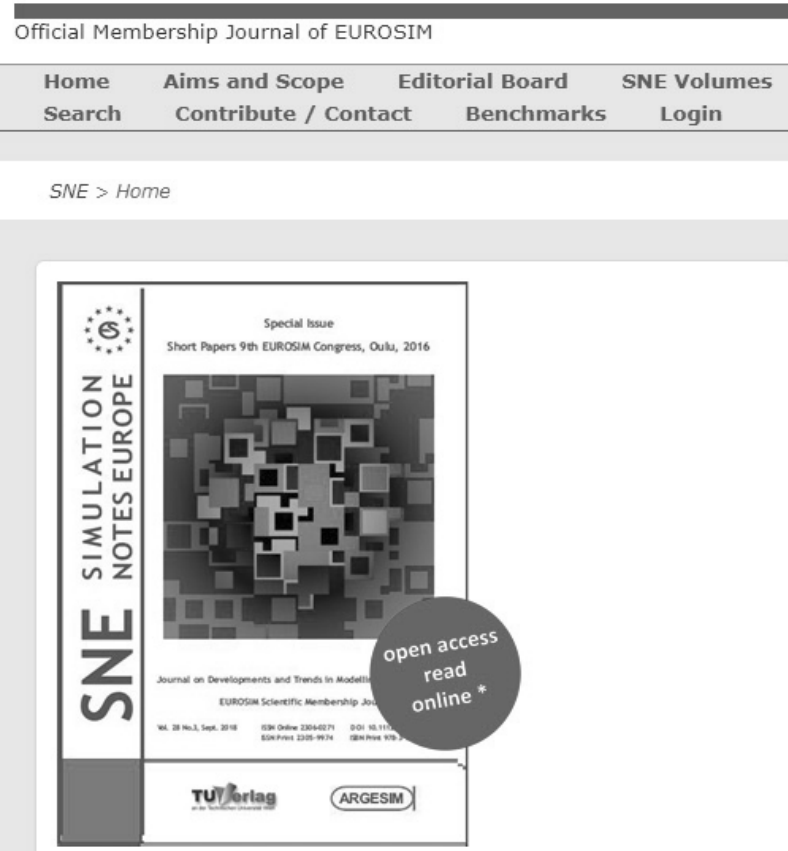

SNE 28(3), Special Issue "Short Papers 9th EUROSIM Congress, Oulu, 2016", September 2018

DOI: $10.11128 /$ sne.28.3.1042

ISSN 2306-0271

Contents:

- Monitoring and Control in Mining

- Actuator Fault Tolerant Control for a Rotary Wing Aircraft

- Utilizing Optical Monitoring to Predict the Effluent Quality in the Activated Sludge Processes

- Fault Tolerant Control for a Rotary Wing Aircraft

- Simplified Mechanistic Model of the Multiple Hearth Furnace

\section{\&*EUROSIM$$
\text { Federation of European Simulation Societies }
$$

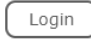

EUROSIM News Events Societies Journal SNE Publications TCs Contact Internal

EUROSIM > Publications

In order to improve dissemination of publication in modelling and simulation, EUROSIM provides download of Proceeding., Abstract Volumes and other some basic publications are open access, but full Proceedings and extended publications can be accessed by members of the EUROSIM societies (please use your EUROSIM Society Login - use Login Button right above, you will be redirected to this page)

Proceedings EUROSIM Congresses - Conferences MATHMOD 2018 Extended Abstract Volume

ARGESIM Publisher, 2018, ARGESIM Report no. 55, ISBN: 978-3-90160891-9 (ebook), DOI: 10.11128/arep.55, (AM 166)

Read more

6th UBT Conference 2017 - KA-SIM 


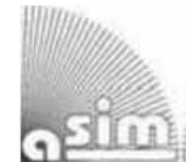

\section{ASIM \\ German Simulation Society Arbeitsgemeinschaft Simulation}

ASIM (Arbeitsgemeinschaft Simulation) is the association for simulation in the German speaking area, servicing mainly Germany, Switzerland and Austria. ASIM was founded in 1981 and has now about 400 individual members (incl. associated), and 90 institutional or industrial members.

$\rightarrow$ www.asim-gi.org with members' area

奉”info@asim-gi.org,admin@asim-gi.org

$\bowtie$ ASIM - Inst. of Analysis and Scientific Computing

TU Wien - Technical Univ. Vienna

Wiedner Hauptstrasse 8-10, 1040 Vienna, Austria

ASIM is organizing / co-organizing the following international conferences:

- $\quad$ ASIM Int. Conference Simulation in Production and Logistics - biannual - ASIM SPL Conference

- ASIM Symposium Simulation Techniques - biannual - ASIM SST Conference

- MATHMOD Int. Vienna Conference on Mathematical Modelling - triennial

- EUROSIM Congress - triennial

Furthermore, ASIM is co-sponsor of WSC - Winter Simulation Conference, of SCS Simulation Conferences, and of I3M conference series.

ASIM is structured in ASIM Sections, dealing with various methods and applications. Within the sections or across sections, working groups discuss and document new developments.

Past Events. In 2018 ASIM has successfully organized the ASIM Symposium Simulation Techniques in October in Hamburg, and has co-organized the MATHMOD 2018 Conference in February in Vienna. Furthermore, the ASIM Sections have organized workshops on their topics. Proceedings and Workshop Reports are available via ASIM website, EUROSIM website, and contributions to SNE Special Issues via the SNE website.

\section{Coming Events}

Every two years the ASIM Int. Conference Simulation in Production and Logistics is organized. ASIM SPL 2019 is ASIM's main event in 2019:

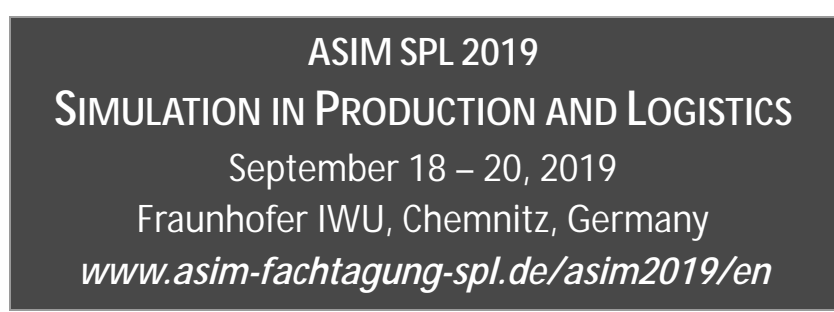

ASIM SPL is at present Europe's largest conference on simulation in production and logistics, presenting future-oriented trends and current developments, scientific works as well as interesting industry applications. Discussed topics cover a range of issues from matters of resource efficiency and digitalisation through to virtual assistance as well as further diverse aspects that relate to the application of simulation for planning, commissioning and operating factory and logistics systems. Intentionally, reports from research and teaching are presented equitably alongside developments and industrial applications.

This constellation allows the conference to be an ideal platform to interact with top-class speakers and participants with a research or an application background, to gather insights on possible applications of simulation, to establish professional contacts and to survey new offerings. Workshops and tutorials, an evening dialogue event as well as the accompanying exhibition with software vendors and service providers from the simulation industry create additional opportunities for detailed discussions. For detailed information, visit:

www.asim-fachtagung-spl.delasim2019/en/

In 2019, the ASIM Sections organise workshops, with contributions in English or German language; main workshop events are:

ASIM GMMS/ STS 2019
Workshop of ASIM Sections Foundation in M od\&Sim
and Simulation of Technical Systems
February 21 - 22, 2019, DLR Braunschweig, Germany
asim2019stsgmms. besl-eventservice.de

ASIM SUG 2019
Workshop of ASIM Section Simulation in
Environmental Systems
May 8 -9, 2019, Fraunhofer IEE Kassel, Germany
enviroinfo.eu/ de/ asim-workshop-2019

ASIM members will prepare contribution for other main international simulation conferences:

\section{EUROSIM CONGRESS, JULY, LA RIOJA, SPAIN www.eurosim2019.com}

\section{SPRINGSIM, APRIL 2019, TUCSON, ARIZONA, USA SUMMERSIM, JULY 2019, BERLIN, GERMANY I3M, SEPTEM BER 2019, LISBON, PORTUGAL Links: www.asim-gi.org}

ARGESIM Benchmarks. ASIM Sections GMMS/STS are preparing a new ARGESIM Benchmark on concurrent event handling in simulation - info ASIM GMMS.

F. Breitenecker, ASIM President 


\section{DUTCH BENELUX}

\section{SIMULATION SOCIETY}

General Information. The

Dutch Benelux Simulation

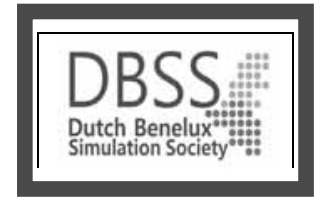

Society (DBSS) was founded in July 1986 in order to create an organization of simulation professionals within the Dutch language area. The DBSS objective is to promote the correct application of modelling and simulation techniques. The society aims to be a meeting platform for companies and academics with modelling and simulation as a common denominator.

$\rightarrow$ www.dutchbss.org

棒p p.m.scala@hva.nl

$\triangle$ Dutch Benelux Simulation Society

Mekelwek 4, 2628CD Delft, The Netherlands

General Activities. DBSS promotes the following activities related with simulation and/or modelling:

- $\quad$ Organization of the yearly DBSS Symposium

- Organization of courses and workshops

- Student exchanges via partner societies in Europe and the rest of the world

- $\quad$ Academic exchanges between participating universities

- $\quad$ Building bridges between industry and experts

- $\quad$ Publication of reports

- Development and distribution of material for education

\section{Past Events.}

DBSS Symposium: The DBSS Symposium 2018: Simulation in Practice, was held the $23^{\text {rd }}$ of November in Amsterdam, hosted by the Amsterdam School of International Business (AMSIB). Several speakers gave talks about different simulation applications to real world issues covering different areas. A special session was reserved for PhD students that presented their works about the use of simulation techniques. Representatives from universities, industries, and practitioners attended the symposium giving their contributions by discussing, sharing ideas and views about the use of simulation techniques.

\section{Various}

The DBSS will present its candidacy for the EUROSIM 2022 to be held in Amsterdam in July 2022.

M. Mujica Mota,m.mujica.mota@hva.nl

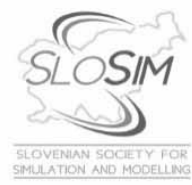

\section{SLOSIM - Slovenian Society for Simulation and Modelling}

$\rightarrow$ www.slosim.si $\quad$ 奉- slosim@fe.uni-lj.si

$\triangle \quad$ SLOSIM / Vito Logar, Faculty of Electrical

Engineering, University of Ljubljana,

Tržaška 25, 1000 Ljubljana, Slovenia

News: On Dec. 4. 2018 SLOSIM had its annual meeting. A new board was elected:

- Vito Logar, president

- Božidar Šarler, vice president

- Simon Tomažič, secretary

- Milan Simčič, treasurer

- Borut Zupančič, EUROSIM representative

Activities: The activities of the society during the last election period were described during the meeting:

- Several organizations of LEGO Masters tournament.

- Several lectures: Modelling of Fuel Cells - From Concepts to Applications, Prof. Cesare Pianese, University Salerno, Italy;

Petri nets, systems and control, Prof. Gašper Mušič, University of Ljubljana, Faculty of Electrical Engineering; Magnetic control of a tokamak plasma: an introduction, Prof. Marco Ariola, University of Naples; Automated bioreactor feeding, Dr. Simon Tomažič, University of Ljubljana, Faculty of Electrical Engineering.

- $\quad$ Active participations, track organizations on conferences EUROSIM, MATHMOD, I3M, ERK, IEEE EAIS

- Keynote lecture on EUROSIM 2016 Oulu: Ass. Prof. Vito Logar: Modelling and simulation of the electric arc furnace processes.

- Renovation of SLOSIM web page www.slosim.si and logo.

- SNE: special issue 'Modelling and simulation in modern control engineering', guest editor Ass. Prof. Vito Logar, several papers were written by SLOSIM members.

- Many activities in the ERASMUS + project INMOTION (Innovative teaching and learning strategies in open modelling and simulation environment for student-centered engineering education). For the topic Modelling and simulation in control engineering SLOSIM members wrote a book, a publication of examples and also developed materials for a MOOC (massive open on-line course). They organized the retraining for 40 members in Ljubljana and participated in a Summer school in Sankt Peterbourg.

- Activities also in the CEPUUS program (Modelling, Simulation and Computer-aided Design in Engineering and Management) - organization and implementation of a Summer school.

- Borut Zupančič, the representative in the EUROSIM board reported about EUROSIM activities and preparations for the next $10^{\text {th }}$ congress EUROSIM 2019, 1. - 5. July 2019, Logroño, La Rioja, Spain.

Borut Zupančič, borut.zupancic@fe.uni-lj.si 


\section{EUROSIM 2019 LA RIOJA (SPAIN), JULY $1^{\text {ST }} 5^{\text {TH }}$ $10^{\text {th }}$ Congress of the Federation of European Simulation Societies}

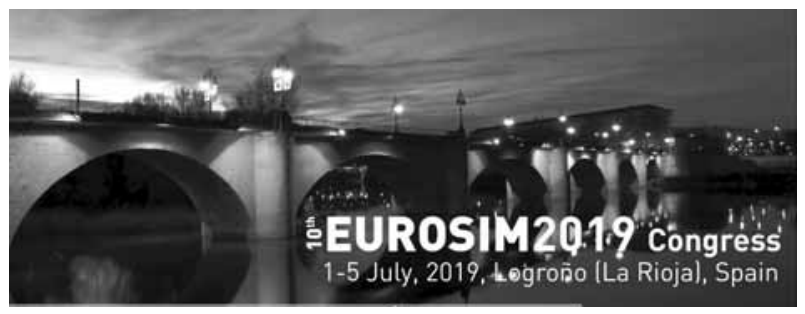

Presentation and CfP. EUROSIM Congresses are the most important modelling and simulation events in $\mathrm{Eu}-$ rope. For EUROSIM 2019, we are soliciting original submissions describing novel research and developments in the following (and related) areas of interest: Continuous, discrete (event) and hybrid modelling, simulation, identification and optimization approaches.

Two basic contribution motivations are expected: M\&S Methods and Technologies and M\&S Applications. Contributions from both technical and non-technical areas are welcome.

Congress Topics. The EUROSIM 2019 Congress will include invited talks, parallel, special and poster sessions, exhibition and versatile technical and social tours. The Congress topics of interest include, but are not limited to: Intelligent Systems and Applications

Hybrid and Soft Computing, Data \& Semantic Mining

Neural Networks, Fuzzy Systems \& Evolutionary Computation

Image, Speech \& Signa! Processing

Systems Intelligence and Intelligence Systems

Autonomous Systems

Energy and Power Systems

Mining and Metal Industry, Forest Industry

Buildings and Construction

Communication Systems

Circuits, Sensors and Devices

Security Modelling and Simulation

Bioinformatics, Medicine, Pharmacy and Bioengineering

Water and Wastewater Treatment, Sludge Management and Biogas

Condition monitoring, Mechatronics and maintenance

Automotive applications

e-Science and e-Systems

Industry, Business, Management, Human Factors and Social Issues Virtual Reality, Visualization, Computer Art and Games

Internet Modelling, Semantic Web and Ontologies

Computational Finance \& Economics

Simulation Methodologies and Tools

Parallel and Distributed Architectures and Systems

Operations Research, Discrete Event Systems

Manufacturing and Workflows

Adaptive Dynamic Programming and Reinforcement Learning Mobile/Ad hoc Wireless networks, mobicast, sensor placement, target tracking

Control of Intelligent Systems. Robotics, Cybernetics, Control

Engineering, \& Manufacturing

Transport, Logistics, Harbour, Shipping and Marine Simulation

\section{Important Dates.}

$\begin{array}{lr}\text { submission deadline } & 10^{\text {th }} \text { March } \\ \text { acceptance deadline } & 1^{\text {st }} \text { April } \\ \text { final submission deadline } & 1^{\text {st }} \text { May }\end{array}$

Congress Venue \& Social Events. The Congress will be held in the City of Logroño, Capital of La Rioja (Spain) very well-known by the Rioja red wines. The main venue and the exhibition site is the University of La Rioja (UR), located on a modern campus in Logroño, capital of La Rioja, where 7500 students are registered. The UR is the only public University in this small, quiet region in Northern Spain.

La Rioja offers three main points of interest to its visitors:

- The wine and the cellars. La Rioja was the first region with qualified designation of origin and is a World reference for the red wines quality. Its wineries combine modernity with history and tradition, and the wine museum is the world's largest dedicated to this food. Wine is the centre of La Rioja's culture, economy and society.

- The monasteries, among which are Cañas, Valvanera, Santa Maria La Real, and of course the San Millán de la Cogolla monasteries (Suso and Yuso), cradle of the first words written in the Spanish language, included in UNESCO's World Heritage List in 1996.

- The Santiago's Road, which for centuries has made constant and continuous the visit of pilgrims to the city, where they are always welcome. Logroño is one of the main cities of the main Santiago's road in Spain, known as the French route (Jaca, Pamplona, Logroño, Burgos, León, Astorga, Santiago).

In addition to these attractions of La Rioja, Logroño, its capital, with a historical helmet with four remarkable religious monuments (Santa María La Redonda, Santiago, San Bartolomé and Santa María de Palacio), is well known for Laurel Street, the most famous Street of Tapas in Spain.

Of course, social events will reflect these interest points, as can be verified in the Program.

Additionally, the situation in Logroño allows visits to wonderful environments less than 2 hours away (San Sebastian, Burgos, Bilbao, Zaragoza, Pamplona, etc.).

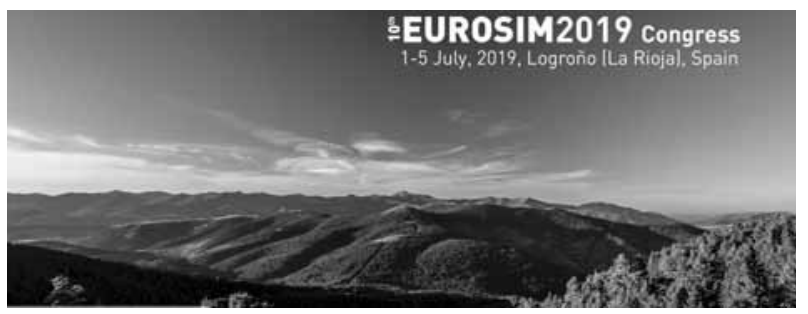

SNE 28(4) - 12/2018 N 13 


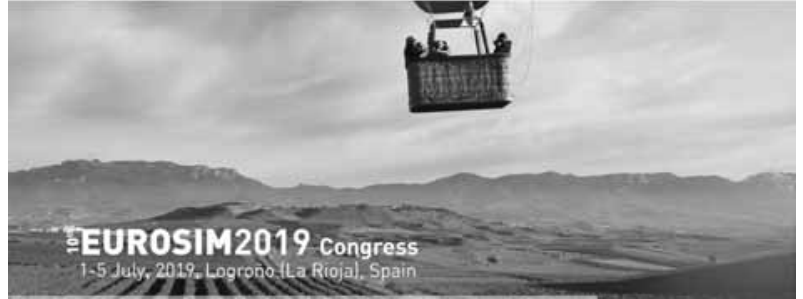

\section{Program \& Scheduling}

The Congress includes three days of work (Tuesday to Thursday) combined with cultural and social activities, a previous day of simulation courses and pre-reception (Monday), a day devoted to cultural and technical activities (Friday), and finally, as an extension, the possibility of a trip (Saturday) to the festivities of San Fermines in Pamplona. All this is detailed in the Programme:

\section{Monday $\mathbf{1}^{\text {st }}$ July}

Morning: Free courses on continuous and discrete events simulation.

Lunch and coffee breaks

Evening: Dinner and Rioja Wine Tasting Course

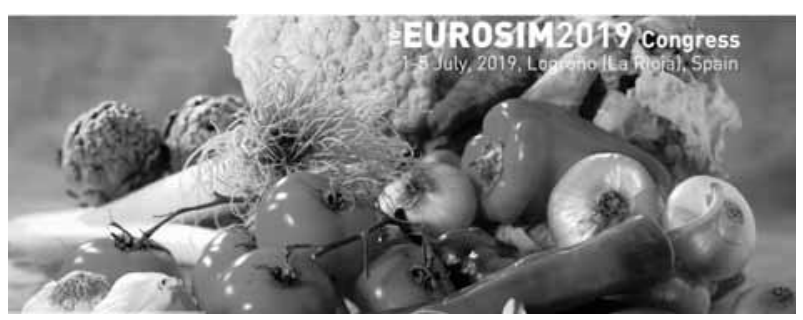

\section{Tuesday $2^{\text {nd }}$ July}

Morning: Plenary sessions. Reception at the city hall.

Lunch and coffee breaks

Afternoon: Parallel sessions (optional visit to the Wine Museum for those who cannot visit it on Saturday)

Evening: Dinner in the old town

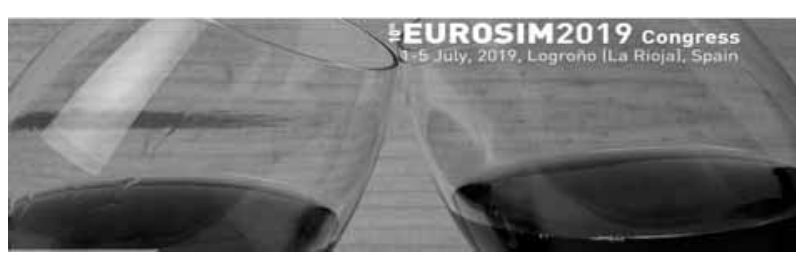

\section{Wednesday $3^{\text {rd }}$ July}

Morning: Parallel sessions

Lunch and coffee breaks

Afternoon: Parallel sessions

Evening: Walk for the Santiago's road in the city to the Gala Dinner in a Winery (and visit to the winery)

\section{Thursday $4^{\text {th }}$ July}

Morning: Parallel sessions

Lunch and coffee breaks

Afternoon: Parallel sessions

Evening: Guided visit to the old town and Dinner of typical tapas at bars of Laurel Street

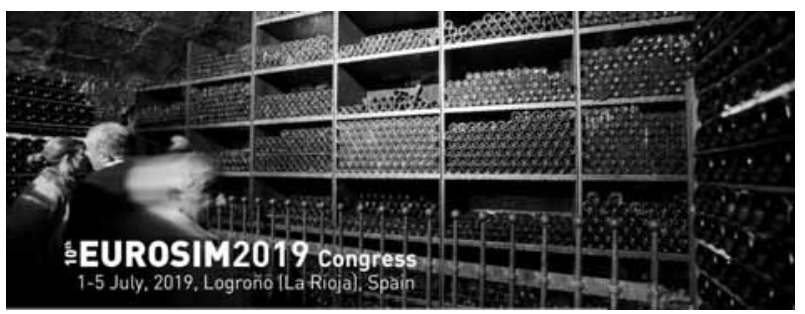

\section{Friday $5^{\text {th }}$ July}

Technical and Cultural visits, including the Wine Museum, Wineries (such as the famous Marques de Riscal, of architect Frank Gehry), and the well-known monasteries (Suso, Yuso, Cañas, Santa María La Real, San Millán, and Valvanera)

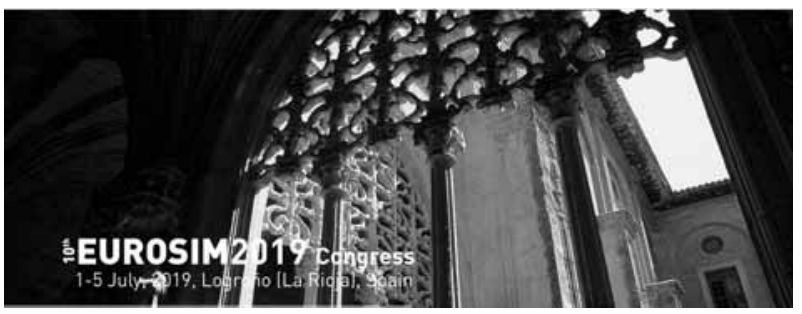

\section{Saturday $6^{\text {th }}$ July}

As an extension of the activities, and taking advantage from the vicinity of Pamplona, the well-known city for the festivities of San Fermines and the bullfights for its streets during the festivities, immortalized by Hemingway, there will be an excursion to the event of the official beginning of the festivity, with "el chupinazo" (rocket announcer).

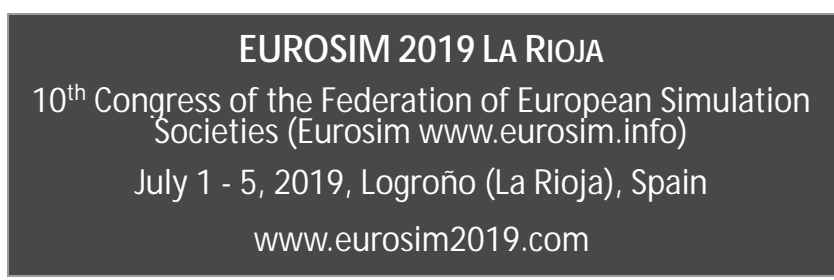

Emilio Jiménez, EUROSIM President emilio.jimenez@unirioja.es 


\section{Parlez-vous}

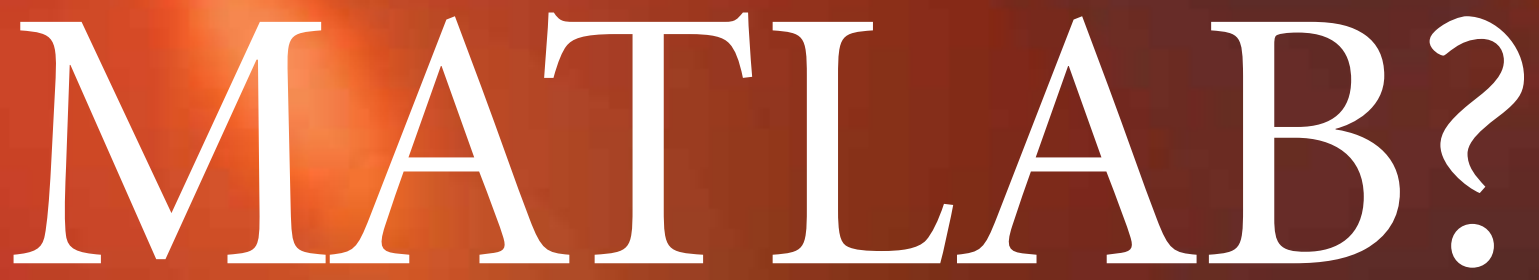

Über eine Million Menschen weltweit sprechen

MATLAB. Ingenieure und Wissenschaftler in

allen Bereichen - von der Luft- und Raumfahrt

über die Halbleiterindustrie bis zur Bio-

technologie, Finanzdienstleistungen und

Geo- und Meereswissenschaften - nutzen

MATLAB, um ihre Ideen auszudrücken.

Sprechen Sie MATLAB?

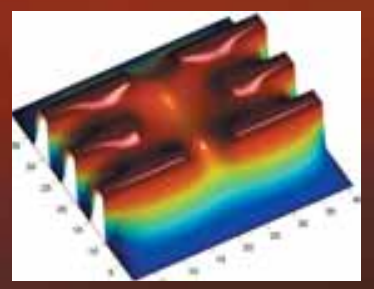

Modellierung eines elektrischen

Potentials in einem Quantum Dot.

Dieses Beispiel finden Sie unter:

www.mathworks.de/ltc

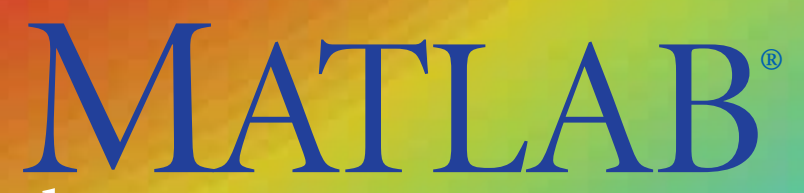

The language of technical computing 


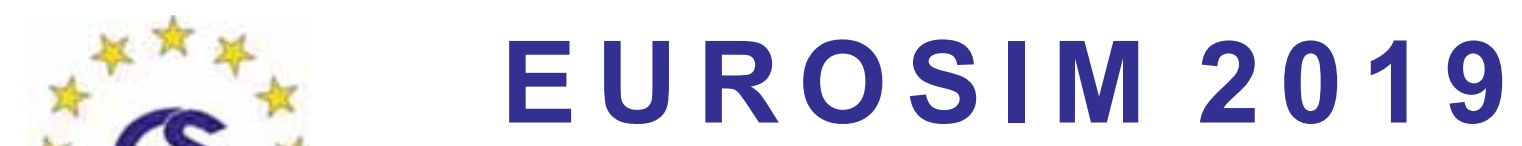

$10^{\text {th }}$ EUROSIM Congress on Modelling and Simulation La Rioja, Logroño, Spain, July 1 - 5, 2019
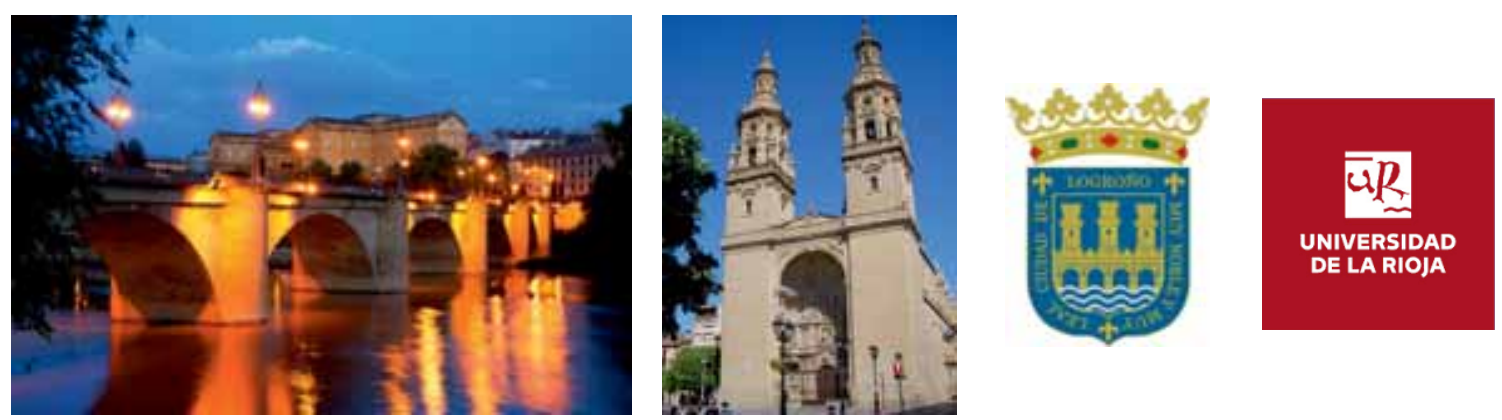

EUROSIM Congresses are the most important modelling and simulation events in Europe. For EUROSIM 2019, we are soliciting original submissions describing novel research and developments in the following (and related) areas of interest: Continuous, discrete (event) and hybrid modelling, simulation, identification and optimization approaches. Two basic contribution motivations are expected: M\&S Methods and Technologies and M\&S Applications.

Contributions from both technical and non-technical areas are welcome.

Congress Topics The EUROSIM 2019 Congress will include invited talks, parallel, special and poster sessions, exhibition and versatile technical and social tours. The Congress topics of interest include, but are not limited to:

Intelligent Systems and Applications Hybrid and Soft Computing Data \& Semantic Mining Neural Networks, Fuzzy Systems \& Evolutionary Computation Image, Speech \& Signal Processing Systems Intelligence and Intelligence Systems Autonomous Systems Energy and Power Systems Mining and Metal Industry Forest Industry Buildings and Construction Communication Systems Circuits, Sensors and Devices Security Modelling and Simulation
Bioinformatics, Medicine, Pharmacy and Bioengineering

Water and Wastewater Treatment, Sludge Management and Biogas Production

Condition monitoring, Mechatronics and maintenance

Automotive applications

e-Science and e-Systems

Industry, Business, Management, Human Factors and Social Issues

Virtual Reality, Visualization, Computer Art and Games

Internet Modelling, Semantic Web and Ontologies

Computational Finance \& Economics
Simulation Methodologies and Tools Parallel and Distributed Architectures and Systems Operations Research

Discrete Event Systems Manufacturing and Workflows Adaptive Dynamic Programming and Reinforcement Learning

Mobile/Ad hoc wireless networks, mobicast, sensor placement, target tracking Control of Intelligent Systems Robotics, Cybernetics, Control Engineering, \& Manufacturing Transport, Logistics, Harbour, Shipping and Marine Simulation

Congress Venue / Social Events The Congress will be held in the City of Logroño, Capital of La Rioja, Northern Spain. The main venue and the exhibition site is the University of La Rioja (UR), located on a modern campus in Logroño, capital of La Rioja, where 7500 students are registered. The UR is the only University in this small, quiet region in Northern Spain. La Rioja is where the Monasteries of San Millán de la Cogolla, cradle of the first words written in the Spanish language, are situated, sites included in UNESCO's World Heritage List in 1996. Of course, social events will reflect this heritage - and the famous wines in la Rioja.

Congress Team: The Congress is organised by CAE CAE-SMSG, the Spanish simulation society, and Universidad de la Rioja.

Info: Emilio Jiménez, EUROSIM President, emilio.jimenez@unirioja.es 